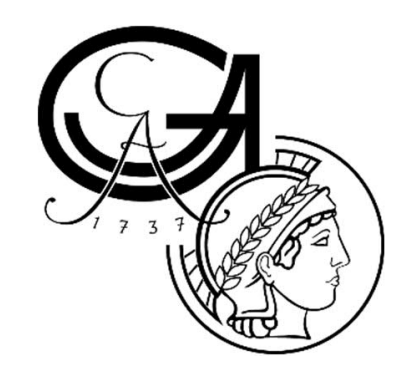

\title{
Molecular physiology of sound encoding
}

\author{
Dissertation \\ for the award of the degree \\ "Doctor rerum naturalium" \\ of the Georg-August-Universität Göttingen \\ within the doctoral program \\ "International Max Planck Research School for Neuroscience" \\ of the Georg-August University School of Science (GAUSS)
}

submitted by

Lina María Jaime Tobón

born in

Medellín, Colombia

Göttingen, 2019 



\section{Members of the Thesis Advisory Committee:}

\section{Prof. Dr. Tobias Moser}

Institute for Auditory Neuroscience \& InnerEarLab, University Medical Center Göttingen Prof. Dr. Dr. h.c. Erwin Neher

Department of Membrane Biophysics, Max Planck Institute for Biophysical Chemistry, Göttingen

Prof. Dr. Manfred Lindau

School of Applied and Engineering Physics, Cornell University, USA

\section{Further members of the Examination Board:}

\section{Prof. Dr. Manuela Schmidt}

Somatosensory Signaling and Systems Biology Group, Max-Planck-Institute of Experimental Medicine, Göttingen

Prof. Dr. Thomas Dresbach

Department of Anatomy and Embryology, Georg-August University, Göttingen

Prof. Dr. Martin Göpfert

Dept. of Cellular Neurobiology, Schwann-Schleiden Research Centre, Göttingen

Date of oral examination: 24.10 .2019 



\section{Declaration}

I hereby declare that this thesis has been written independently and with no other sources and aids than quoted.

Lina María Jaime Tobón

Göttingen, 20. September, 2019 

A mi Papá y la teoría del columpio 



\section{Contents}

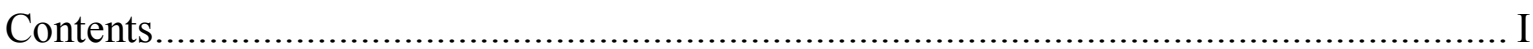

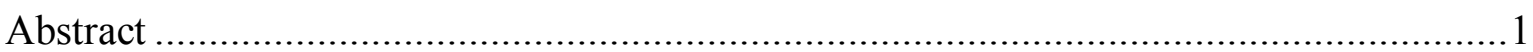

Chapter 1 General introduction ........................................................................... 3

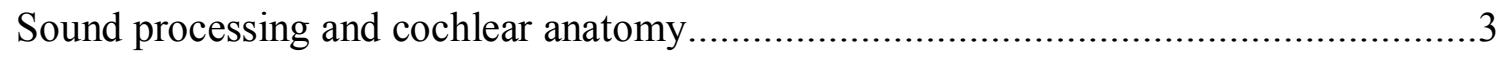

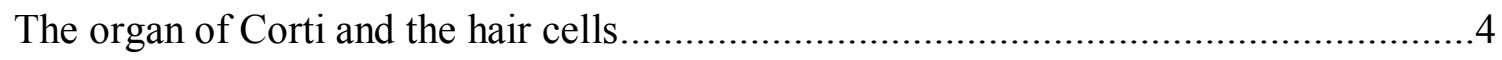

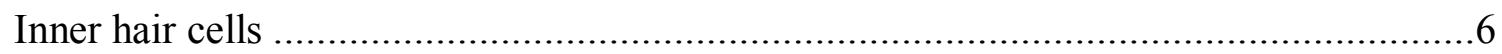

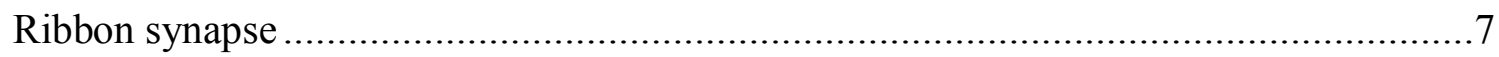

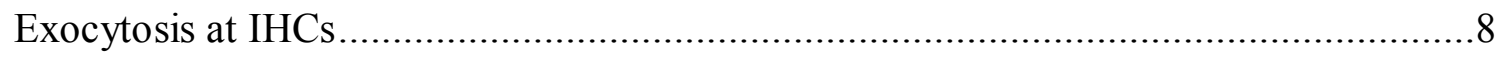

Unconventional synaptic release machinery of IHCs ..........................................

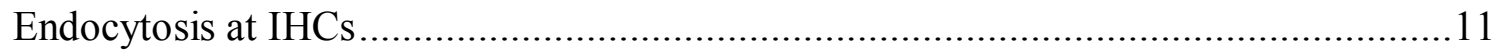

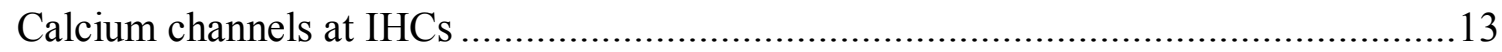

Spiral ganglion neurons and their in vivo response properties .................................. 15

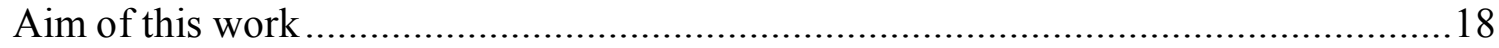

Chapter 2 Biophysical properties of inner hair cell ribbon synapses at near physiological

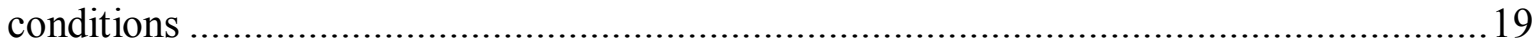

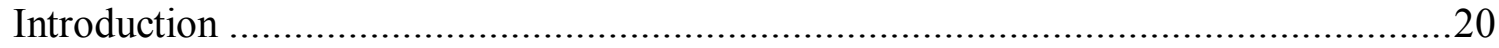

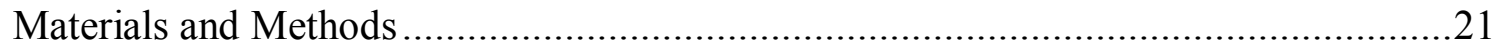

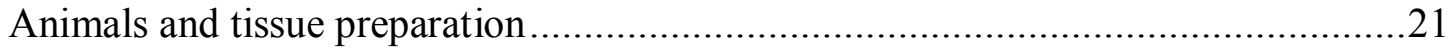

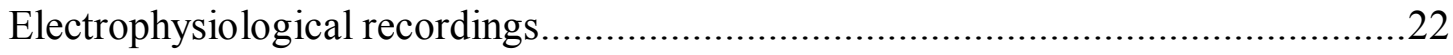

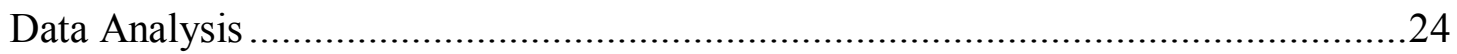

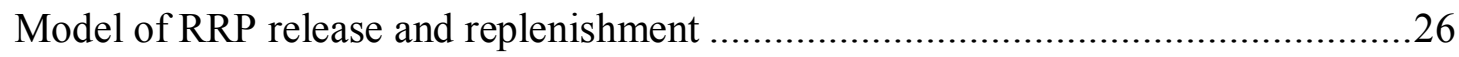

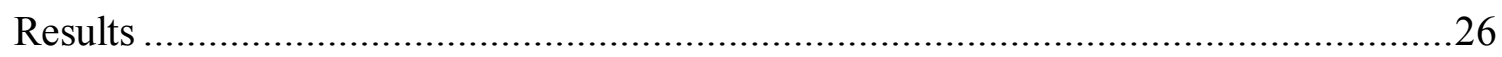

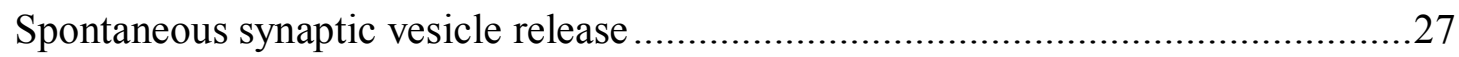

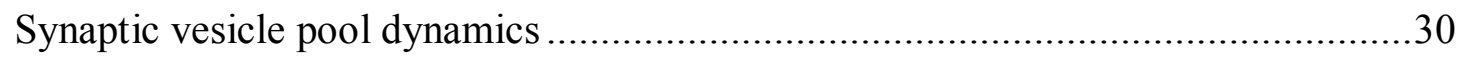

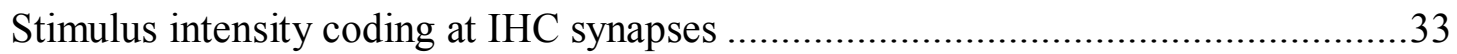

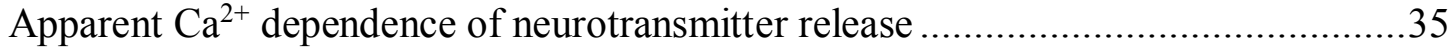


Discussion 39

Pillar synapses have higher rates of spontaneous release ................................... 40

Distinct release modes at low and high SR synapses .......................................... 41

Synaptic vesicle pool dynamics at individual active zones ................................ 43

Nanodomain control of exocytosis at murine IHCs ........................................... 44

Chapter 3 The synaptic ribbon is critical for sound encoding at high rates and with temporal

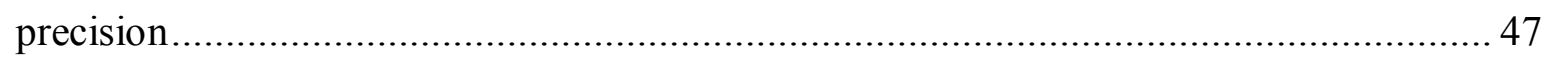

Chapter 4 Endophilin-A regulates presynaptic $\mathrm{Ca}^{2+}$ influx and synaptic vesicle recycling in

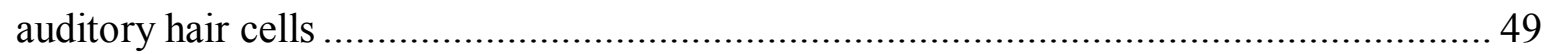

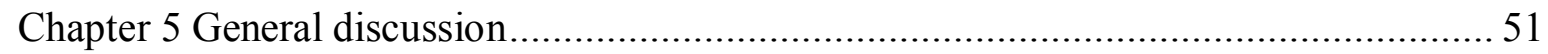

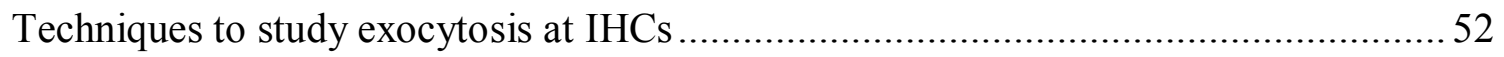

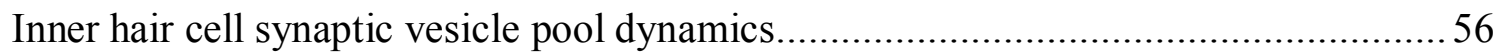

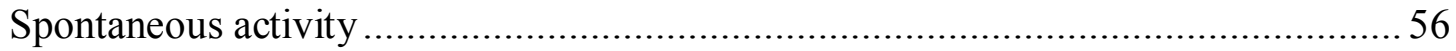

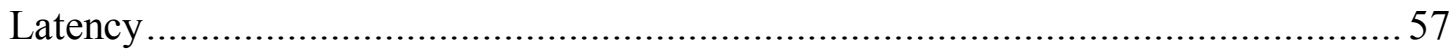

Synaptic depression, spike rate adaptation and vesicle replenishment.....................59

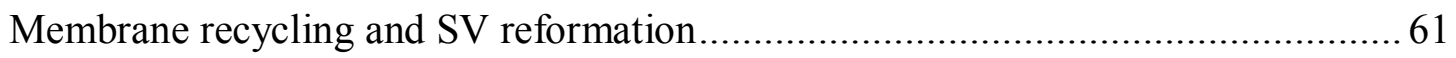

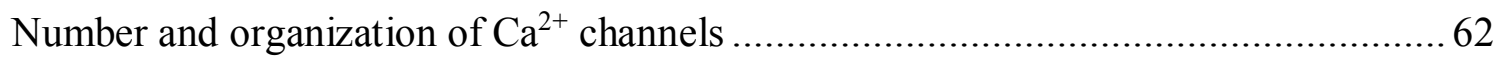

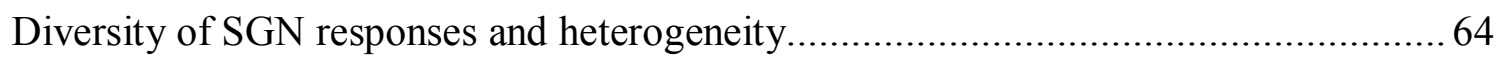

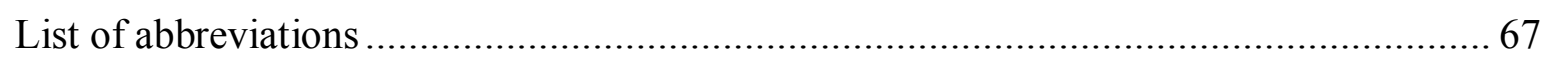

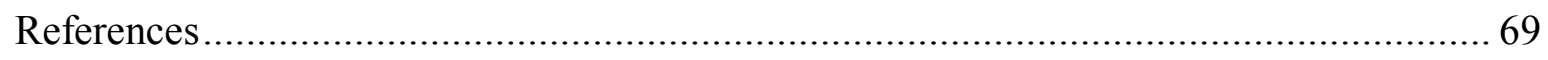

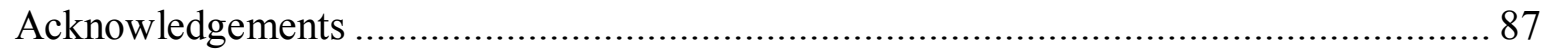

Appendix

The synaptic ribbon is critical for sound encoding at high rates and with temporal precision

Endophilin-A regulates presynaptic $\mathrm{Ca} 2+$ influx and synaptic vesicle recycling in auditory hair cells 90 


\section{Abstract}

Inner hair cells (IHC) are responsible for transforming mechanical sound-borne vibrations into electrical signals and conveying this information to the afferent spiral ganglion neurons (SGNs). Upon stimulation, the receptor potential triggers the opening of voltage-gated $\mathrm{Ca}^{2+}$ channels, mediating the fusion of vesicles and the consequent release of neurotransmitter from the presynaptic active zone to the postsynaptic bouton. In vivo recordings from SGNs revealed highly synchronized onset responses and indefatigably sustained firing rates. A plethora of techniques has been used to understand how IHCs accomplish this impressive performance of neurotransmitter release, and which mechanisms establish the diversity of auditory nerve fibers (ANFs) responses to stimulation. This thesis provides further insight into the molecular physiology of sound encoding.

First, synaptic transmission at individual murine IHC afferent synapses was studied using paired IHC-bouton patch clamp recordings in near physiological conditions. Synapses contacting the pillar side of the IHC had higher rates of spontaneous EPSCs that were characterized by larger amplitudes yet similar charges. High spontaneous rates (SR) synapses had significantly lower voltage thresholds of release and tended to have shorter synaptic delays, as well as faster recovery from readily releasable pool (RRP) depletion. Furthermore, this study corroborated that a $\mathrm{Ca}^{2+}$-nanodomain-like control of exocytosis operates at IHCs synapses.

Second, collaborators and I studied synaptic transmission in IHC synapses from RIBEYE knockout $(\mathrm{KO})$ mice. Their ribbonless synapses were characterized by several small active zones opposing each postsynaptic density. In vivo ANFs recordings revealed an impaired synaptic transmission, characterized by lower spontaneous and evoked firing rates, lower temporal precision and a slower recovery from adaptation. $\mathrm{Ca}^{2+}$ imaging of individual active zones showed that the $\mathrm{Ca}^{2+}$ channels required more depolarized potentials to activate. Consequently, weak depolarizations during perforated patch-clamp recordings resulted in reduced exocytosis compared to wildtype (Wt). We postulated a role of the ribbon in synaptic vesicle replenishment and $\mathrm{Ca}^{2+}$ channel regulation.

Third, collaborators and I studied the role of endophilin-A1-3, endocytic adaptor proteins, in IHCs. Perforated patch-clamp recordings from organotypic cultures and from explanted organs of Corti revealed lower $\mathrm{Ca}^{2+}$ influx, impaired sustained exocytosis and slower 
endocytic membrane retrieval in Endophilin-A-deficient IHCs. At the ultrastructural level, the IHC active zones had lower counts of synaptic vesicles, but increased numbers of coated structures and endosome-like vacuoles. In addition, we postulated a molecular interaction between endophilin-A1 and otoferlin based on co-immunoprecipitation. We proposed a positive role of endophilin- $\mathrm{A}$ in the modulation of $\mathrm{Ca}^{2+}$ channels, and in synaptic vesicle recycling, likely via coupling of exo- and endocytosis, membrane retrieval, synaptic vesicle uncoating and reformation. 


\section{Chapter 1 General introduction}

The sense of hearing, also known as audition, allows us to experience a wonderful world, from crucial sounds for survival to the emotions triggered by music. Audition shares principles with other senses: it is most sensitive to its specific stimulus, here sound pressure changes, and bears receptor cells, the mechanosensory hair cells transducing the stimulus. In the following sections, I will give a brief introduction into the auditory system with a focus on the cochlea and its sensorineural elements, covering aspects of the anatomy, physiology and molecular players.

\section{Sound processing and cochlear anatomy}

Our auditory system analyses acoustic input from the external environment. It captures the sound, efficiently transmits it to the receptor organ, and transduces it into electrical signals that travel to central stages of the auditory system for further processing of sensory information. To perform this task, the ear is equipped with three functional parts: the outer ear, the middle ear and the inner ear |Figure 1|.

Acoustic waves are funneled by the auricle of the outer ear into the external ear canal until they reach the tympanic membrane. The periods of compression and rarefaction of the soundwave cause the tympanic membrane to vibrate back and forth. These vibrations are transmitted to the three osscicles, incus, malleus and stapes, located in the middle ear. The stapes then acts as a piston that compresses the fluid contained in the inner ear, allowing the transformation of airborne vibrations into fluid movements in the cochlea.

The cochlea of the inner ear is a snail-shaped bony structure with three liquid-filled compartments in its interior: the scala vestibuli, the scala tympani and the scala media. The scala vestibule and tympani end with sealed openings called the oval window and the round window, respectively. The two chambers are separated by the cochlear partition along most of their length, but communicate with one another through the helicotrema at the apex. The third compartment, the scala media, lies within the cochlear partition, separated from the scala vestibuli by the Reissner's membrane and from the scala media by the basilar membrane. Upon sound arrival, the stapes pushes the oval window and produces changes in the fluid pressure of the scala vestibuli. The pressure displaces the cochlear partition downwards and consequently, increases the fluid pressure in the scala tympani. Each period 
of compression and rarefaction of a soundwave initiates pressure oscillations in the three compartments that ultimately cause the basilar membrane to resonate (Von Bekesy, 1956).

The mechanical properties of the basilar membrane are crucial for sound processing and analysis. At the apex of the cochlea, the basilar membrane is broader, relatively thin and flaccid, while it progressively becomes narrower, thicker and stiffer toward the base. Because of this variation along the cochlea's length, each sound frequency produces maximal oscillation at a particular position in the basilar membrane. Low frequency sounds evoke movement near the apex, while high frequency sounds produce motion near the base. The arrangement of sound frequency onto the basilar membrane, called tonotopy, is approximately logarithmic. Tonotopy is well-maintained along the auditory pathway, from the auditory nerve, to the midbrain stations and up to the auditory cortex.

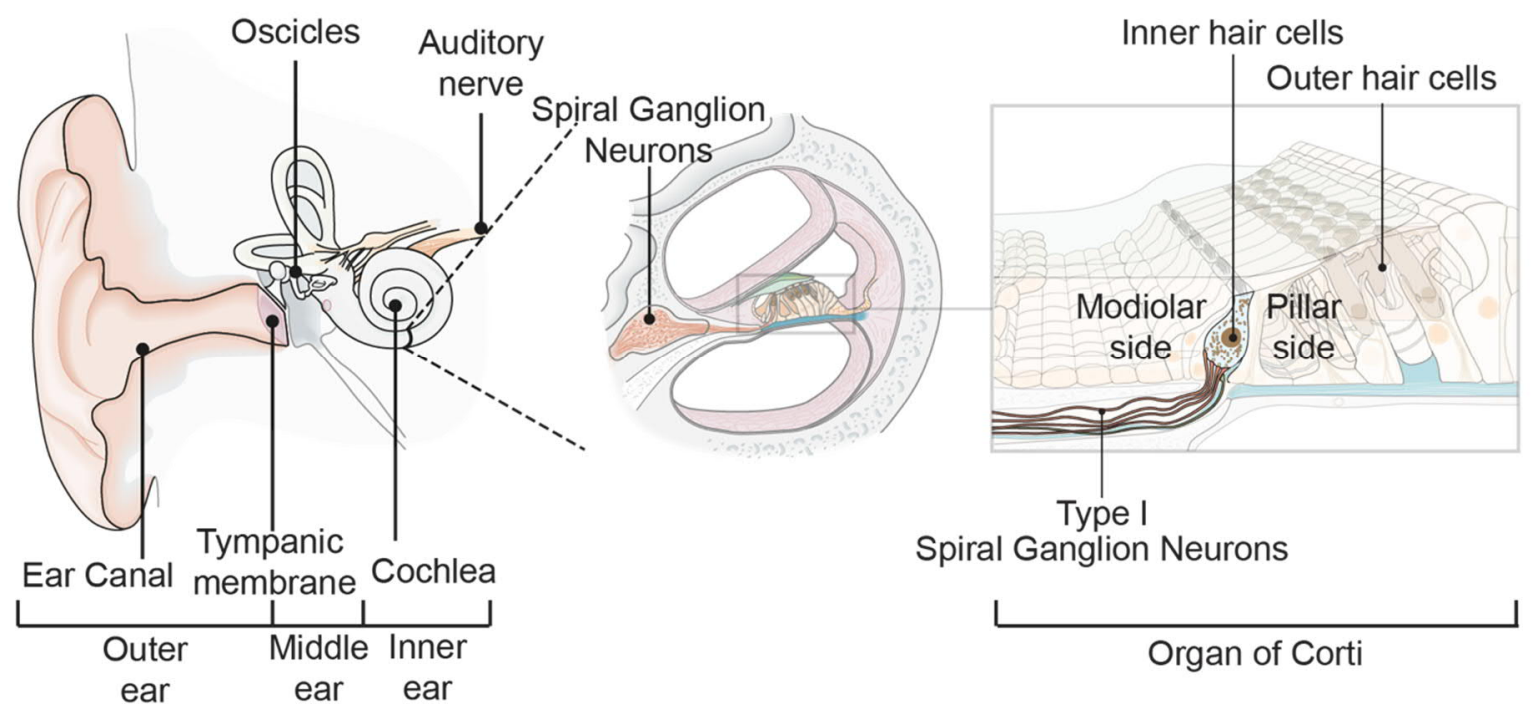

\section{|Figure 1| The ear and the organ of Corti}

Incoming soundwaves arrive at the outer ear, travel through the ear canal and reach the tympanic membrane. The vibration of the tympanic membrane is transmitted to the osscicles, incus, malleus and stapes, present in the middle ear. The stapes then acts as a piston and transmits the sound vibration to the inner ear. Within the sensory epithelium of the auditory system, the organ of Corti, the sound vibration is transduced into a neural code. The organ of Corti is composed of three rows of outer hair cells, one row inner hair cells, the nerve fibers from the spiral ganglion neurons, and supporting and ancillary structures. On the 'pillar side', IHCs face pillar cells, OHCs and Deiters' cells, while on the 'modiolar side', IHCs face the modiolus that contains the SGNs. Illustration modified from (Kandel et al., 2012).

\section{The organ of Corti and the hair cells}

Sitting on the basilar membrane is the organ of Corti, a highly organized epithelium composed of sensory cells, nerve fibers, and supporting and ancillary structures. The sensory 
cells, called hair cells, are organized in three rows of outer hair cells (OHCs) and one row of inner hair cells (IHCs), with two rows of pillar cells separating them. Deiters's and phalangeal cells support the base of the OHCs and IHCs, respectively |Figure 1|. Afferent nerve fibers (ANFs) from the spiral ganglion neurons (SGNs) contact the hair cells at their basal pole.

The hair cells derive their name from the presence of a set of stereocilia in their apical surface. These modified microvilli are organized in three rows of increasing height in a staircase fashion, with a more linear arrangement in IHCs and a V-shape configuration in OHCs. The stereocilia protrude into the endolymph solution contained in the scala media, where they serve as receptors for mechanical movements. In addition, the top of the OHC's hair bundles is embedded in the tectorial membrane, an acellular matrix that lies above the organ of Corti. Each stereocilium is composed of cross-linked actin and adjacent stereocilia are connected at their apex by cadherin structures called tip links (Pickles et al., 1984; Siemens et al., 2004; Söllner et al., 2004). At the lower end of each tip link, two mechanotransducer (MET) channels sit in the tip of the first and second row of stereocilia (Assad et al., 1991; Beurg et al., 2009; Howard and Hudspeth, 1987; Jaramillo and Hudspeth, 1991; Lumpkin and Hudspeth, 1995). The oscillation of the basilar membrane in response to sound leads to movements of the tectorial membrane and of the endolymph in the space beneath it. These movements deflect the stereociliary bundles of OHCs and IHCs and thus, initiate the mechanoelectrical transduction process. Deflection toward the taller stereocilia causes the activation of MET ion channels and the consequent depolarization of the hair cell due to the increase in cation influx (mostly $\mathrm{K}^{+}$) from the endolymph (Corey and Hudspeth, 1979). In contrast, deflection toward the shorter stereocilia causes MET channels to close, thereby hyperpolarizing the cell. With a sinusoidal stimulus, the opening and closing of the MET channels produces an oscillatory receptor potential in the hair cells (Palmer and Russell, 1986).

The receptor potential consists of a phasic component (AC component) and a continuous component (DC component). The predominance of each component varies according to the stimulus frequency (Palmer and Russell, 1986). In response to low frequency tones, IHCs generate a phasic, large and asymmetrical potential dominated mostly by the AC component. Contrary, in response to high frequency tones, the receptor potential is dominated by a positive DC component with an overlapping AC component that decreases in amplitude with 
the stimulus frequency. Both the $\mathrm{AC}$ and $\mathrm{DC}$ component rise linearly with the stimulus intensity and saturate at high sound pressure levels (Russell and Sellick, 1978).

When OHCs are stimulated by relative movements of the tectorial and basilar membrane, their depolarization leads them to primarily amplify sound vibrations with little if any contribution to synaptic sound encoding (Ashmore, 2008, 1987). OHCs elongate or contract in response to changes in their membrane potential. This membrane-based motor process is called electromotility and is mediated by the motor protein prestin (Cheatham, 2004; Zheng et al., 2000). The oscillatory movements accelerate the basilar membrane motion, amplifying the sound-born vibrations and thus enhancing the sensitivity of hearing and sharpening the frequency tuning of the basilar membrane.

\section{Inner hair cells}

The IHCs are the key players to convey the acoustic information to higher auditory areas through the afferent fibers of type I SGNs. $\mathrm{Ca}^{2+}$ influx through L-type voltage gated $\mathrm{Ca}^{2+}$ channels located at the base of the IHC triggers the fusion of synaptic vesicles with the IHC plasma membrane, and the subsequent release of glutamate onto the synaptic cleft. Glutamate then binds the postsynaptic AMPA receptors located in the boutons of SGNs. The ensuing depolarizing current initiates the generation of the action potential in the SGNs.

IHCs are specialized to encode sound. In vivo, IHCs are reported to have resting potentials ranging between -55 and -20 mV (Russell, 1983; Russell and Sellick, 1978). In vitro, however, the resting potential has been determined to be $-58 \mathrm{mV}$ for apical and $-66 \mathrm{mV}$ for basal IHCs (Johnson, 2015). The resting mechanotransducer current (Johnson et al., 2005; $\mathrm{Xu}$ and Lipscombe, 2001) and several basolateral membrane currents - including rapidly activating large conductance $\mathrm{Ca}^{2+}$-activated $\mathrm{K}^{+}(\mathrm{BK})$ currents, small conductance $\mathrm{Ca}^{2+}$ activated $\mathrm{K}^{+}$(SK) currents and negatively activating delayed rectifier current - are responsible to maintain the IHC at its receptor potential (Johnson, 2015; Kharkovets et al., 2006; Oliver et al., 2003). As it will be discussed later on, the relatively positive resting potential of IHCs is a key feature that shapes high rates of neurotransmitter release even in the absence of sound. 


\section{Ribbon synapse}

The IHC synapses are located in the basal pole of the cell. They differ from the conventional synapses in terms of structure, function and molecular machinery. Ultrastructurally, these synapses are characterized by a cytoplasmic electron-dense proteinaceous structure termed the synaptic ribbon [for review see (Pangršič et al., 2012; Safieddine et al., 2012)]. The ribbon is anchored to the active zone by the scaffold protein Bassoon (Jing et al., 2013; Khimich et al., 2005) and tethers numerous vesicles around it [for a review (Chakrabarti and Wichmann, 2019)]. At a molecular level, synaptic ribbons are composed mainly by the proteins RIBEYE (Becker et al., 2018; Jean et al., 2018; Khimich et al., 2005; Lv et al., 2016) and Piccolino (Dick et al., 2003; Regus-Leidig et al., 2014).

In mammals, each IHC ribbon is associated with one unmyelinated ending of a type I SGN (Robertson, 1984), the most abundant type present in the cochlea (95\%) (Berglund and Ryugo, 1987; Kiang et al., 1982). Roughly, 10 to 30 SGNs contact one IHC, but this number varies by species and tonotopy (Fuchs et al., 2003; Kiang et al., 1982; Liberman, 1982; Meyer and Moser, 2010). Imaging studies using electron microscopy of the mature IHC synapses have been of great utility for estimation of the IHC active zone topography. The estimated presynaptic density of about $420 \times 80 \mathrm{~nm}^{2}$ is mostly occupied by one synaptic ribbon tethering on average 40-70 vesicles [for a review (Chakrabarti and Wichmann, 2019)]. Beneath the ribbon, about 11 to 14 vesicles compose the membrane-associated pool that is thought to constitute the readily releasable pool of vesicles. These vesicles are located 20-50 nm away from the plasma membrane with $40 \%$ of them tethered to the plasma membrane with a tether length of 20-25 nm [for a review (Chakrabarti and Wichmann, 2019; Chakrabarti et al., 2018)].

Upon stimulation, glutamate released into the synaptic cleft binds to the postsynaptic AMPA receptors present in the afferent dendrites of the ANFs (Glowatzki and Fuchs, 2002; Ruel et al., 1999). These receptors are composed manily of GluR2/3 and GluR4 subunits (Parks, 2000; Ravindranathan et al., 2000). Other main constituents of the postsynapse are the scaffolding proteins PSD-95 (Davies et al., 2001), Homer (Becker et al., 2018) and Shank1 (Braude et al., 2015). 


\section{Exocytosis at IHCs}

Exocytosis at IHCs has been extensively studied by performing capacitance measurements $\left(\mathrm{C}_{\mathrm{m}}\right)$ during whole-cell patch clamp recordings. During the initial recordings, Moser and Beutner described the IHC synaptic release as bi-phasic with initial high secretory rates followed by a slower exocytosis during prolonged stimulation (Moser and Beutner, 2000). This suggests the presence of two distinct pools of synaptic vesicles. The first fast exponential component of exocytosis presumably reflects the fusion of the readily-releasable pool of vesicles (RRP). Capacitance measurements, paired IHC and bouton patch-clamp recordings, and modeling of in vivo recordings from SGNs have led to RRP estimates of 4 to 45 vesicles depleting with time constants ranging between 3 to $54 \mathrm{~ms}$ (Frank et al., 2010; Goutman and Glowatzki, 2007; Johnson et al., 2005; Moser and Beutner, 2000; Pangrsic et al., 2010; Peterson et al., 2014). The high variability of the estimates might be a reflection of different animal models, recording conditions and/or assumptions of univesicular/multivesicular release at single active zones. It remains clear though, that synaptic transmission in IHCs achieve high onset release rates exceeding thousands of vesicles per second. The abolishment of this first excitatory component in the presence of the fast $\mathrm{Ca}^{2+}$ buffer, BAPTA, but its persistence in the presence of the slow $\mathrm{Ca}^{2+}$ buffer, EGTA, suggests that the RRP is composed of vesicles released in the close proximity to the $\mathrm{Ca}^{2+}$ channels (Moser and Beutner, 2000). The second slow exponential component of exocytosis is suggested to represent sustained release of synaptic vesicles that have been mobilized to the fusion site by replenishment mechanisms. Due to its sensitivity to EGTA, the mobilization and fusion of this second pool might depend on long distance $\mathrm{Ca}^{2+}$ signaling (Moser and Beutner, 2000).

One prominent characteristic of the IHC ribbon synapse is the high variability in the excitatory postsynaptic current (EPSC) amplitude (coefficient of variations: 77-95\%) and waveform in the absence of stimulus (Glowatzki and Fuchs, 2002). The mechanisms behind this phenomenon are under ongoing debate. Initially, it was proposed that the release of multiple vesicles in a more or less coordinated fashion accounted for the variability in the waveforms. Under this hypothesis, monophasic EPSCs with a sharp rise and decay arise from the highly coordinated exocytosis of on average six vesicles, whereas EPSCs with multiple peaks and variable rise and decay times were attributed to uncoordinated release of several vesicles (Glowatzki and Fuchs, 2002). This notion, although with fewer vesicles involved in the average release event, was supported by the higher apparent capacitance of 
elementary events derived from a non-stationary fluctuation analysis based on whole-cell capacitance measurements than the capacitance estimates based on electron microscopy micrographs (Neef et al., 2007). Additionally, or alternatively, variable EPSC waveforms might originate from single vesicles with different glutamate concentrations (Takamori, 2016).

The multiquantal hypothesis seems hard to reconcile with the fact that several vesicles would need to simultaneously fuse at resting conditions when the release probability is low (Chapochnikov et al., 2014). Therefore, an alternative hypothesis was postulated, in which the variability was attributed by the fusion pore dynamics during fusion of a single vesicle with the plasma membrane (Chapochnikov et al., 2014). In this uniquantal hypothesis, large monophasic EPSCs can be explained by the instantaneous release of the neurotransmitter when the vesicle rapidly and fully collapses with the plasma membrane. The flickering of the fusion pore, which results in the varied diffusion rate of the vesicle content to the synaptic cleft, would then account for multiphasic EPSCs (Chapochnikov et al., 2014). Two recent studies using postsynaptic bouton recordings (Huang and Moser, 2018) and low noise cellattached capacitance measurements (Grabner and Moser, 2018) support this hypothesis.

\section{Unconventional synaptic release machinery of IHCs}

The molecular players at ribbon synapses have been under intensive research. The role of the ribbon itself has been one of the major questions regarding these unconventional synapses. It is well known that ribbons contain the unique scaffolding protein RIBEYE (Schmitz et al., 2000). It is transcribed from the same gene as the essential transcription factor CtBP2 (Hildebrand and Soriano, 2002), making RIBEYE-disruption a challenging task.

Researchers have used different techniques to bypass this issue, including studying the natural variation in size and abundance of RIBEYE during hibernation or diurnal cycle (Hull et al., 2006; Mehta et al., 2013), as well as ribbon photoablation (Mehta et al., 2013; Snellman et al., 2011). A common circumventing approach is to disrupt the presynaptic scaffold protein bassoon that anchors the ribbons to the active zones (Dick et al., 2003; Khimich et al., 2005). However, this leads to combinatory effects of bassoon deletion and ribbon loss on the function of the active zone. In zebrafish, morpholino-mediated RIBEYE knockdown led to reduced afferent innervation and postsynaptic densities (Sheets et al., 2011), while frameshift mutations in the two genes encoding for RIBEYE resulted in 
vesicles gathering around ribbon-like structures that lack electron density, called 'ghost ribbons' (Lv et al., 2016). Yet, there was evidence in both studies of an uncomplete abolition of RIBEYE, probably due to the duplicated genome of this animal model.

Only a couple of years ago a complete disruption of RIBEYE was achieved by knockingout the RIBEYE specific exon in mouse (Maxeiner et al., 2016). At the retina level, the resulting ribbonless synapses showed impaired evoked release and looser coupling of the $\mathrm{Ca}^{2+}$ channels to the release sites. This study supported the view of the synaptic ribbon as a conveyor belt that promotes sustained release and helps in the nanodomain positioning of the $\mathrm{Ca}^{2+}$ channels to the readily-releasable synaptic vesicles. Nonetheless, retinal and IHC ribbon synapses are functionally distinct, hence, assessment of the effects of ribbon absence on IHC synaptic transmission and sound encoding is of great importance.

The large cytomatrix protein bassoon is responsible for anchoring the ribbon to the presynaptic density (Khimich et al., 2005). Disruption of bassoon, for instance, leads to reduced pool of membrane-proximal vesicles, smaller and misshaped $\mathrm{Ca}^{2+}$ channel clusters (Frank et al., 2010), and defective ribbon anchoring (Jing et al., 2013). Physiologically, bassoon mutants show reduced spontaneous and sound-evoked firing rates (Buran et al., 2010; Khimich et al., 2005), impaired RRP exocytosis (Khimich et al., 2005) and smaller EPSC amplitudes (Jing et al., 2013), supporting an essential role of Bassoon for normal hearing. Nevertheless, and as mentioned before, some of these findings might originate from the combinatorial effect of bassoon deletion and ribbon mis-anchoring and/or absence.

IHCs do not express the conventional fundamental synaptic proteins such as synaptophysins, synapsins, or complexins (Safieddine and Wenthold, 1999; Strenzke et al., 2009). Essential neurosecretory soluble N-ethylmaleimide-sensitive factor attachment protein receptor (SNARE) proteins (synaptobrevins/VAMPS 1-3, syntaxins 1-3 and SNAP25) seem also to be absent (Nouvian et al., 2011, but see Safieddine and Wenthold, 1999 for a deviating view). Based on the lack of obvious effects of clostridial neurotoxins on exocytosis, no homologous or functionally redundant proteins are thought to be expressed in the IHC ribbon synapse (Nouvian et al., 2011). Priming factors like Munc13 and proteins from the CAPS family, which are critical for transmission at many synapses (Imig et al., 2014; James and Martin, 2013), are also undetected (Vogl et al., 2015). Additionally, the $\mathrm{Ca}^{2+}$ sensors synaptotagmin 1 and 2 are only transiently expressed during early postnatal age but not in mature IHCs (Beurg et al., 2010; Reisinger et al., 2011). 
Instead, Otoferlin is currently the best candidate as a priming factor and vesicular $\mathrm{Ca}^{2+}$ sensor in IHCs (Michalski et al., 2017; Pangrsic et al., 2010). This multi-C2-domain protein is specifically expressed in hair cells and defects in it cause human deafness DFNB9 (Yasunaga et al., 2000). Otoferlin's binding of $\mathrm{Ca}^{2+}$-with $\mathrm{Ca}^{2+}$ binding to most of its $\mathrm{C} 2$ domains-, as well as its $\mathrm{Ca}^{2+}$ dependent interaction with the SNARE complex in vitro (Ramakrishnan et al., 2014), strongly supports its $\mathrm{Ca}^{2+}$ sensor activity. In line with this hypothesis, otoferlindeficient IHCs have almost complete absence of exocytosis despite structurally normal afferent synapses (Roux et al., 2006). On top of its fundamental role in exocytosis, Otoferlin might also mediate efficient release site clearance and synaptic vesicle reformation (Jung et al., 2015).

\section{Endocytosis at IHCs}

IHCs support high release rates. In consequence, they require an efficient and robust endocytotic machinery that allows retrieval of exocytosed membrane, recycling of synaptic vesicle components and that ensures trafficking of synaptic vesicles' membranes (Kamin et al., 2014). The broad distribution and expression of endocytic proteins (Neef et al., 2014), as well as studies using FM dyes (Kamin et al., 2014), indicate an abundant membrane recycling in IHCs during rest and stimulation.

Membrane trafficking microscopy, ultrastructural analysis and electrophysiological studies suggest three complementary modes of membrane retrieval to support the efficient recycling of synaptic vesicles in IHCs. The first mode, denominated rapid endocytosis or "kiss and run endocytosis", occurs predominantly when intracellular calcium levels exceed $15 \mu \mathrm{M}$ (as a reference, a voltage depolarization to $-15 \mathrm{mV}$ presumably increases intracellular calcium concentration to 20-30 $\mu \mathrm{M}$ ) (Beutner et al., 2001). This mode displays a time constant $\sim 250$ $300 \mathrm{~ms}$ (Beutner et al., 2001; Neef et al., 2014) and it is thought to retrieve a significant fraction of membrane within one second, contributing to the homeostasis of membrane trafficking when the stimulus intensity is high (Beutner et al., 2001). The second mode, clathrin-mediated endocytosis (CME), participates to the membrane retrieval regardless the amount of exocytosis (Duncker et al., 2013; Neef et al., 2014) and it is limited at the IHCs basal surface (Griesinger et al., 2002; Revelo et al., 2014). CME in IHCs occurs at a nearly constant rate of 1-2 fF/s (approximately 2-4 synaptic vesicles per s) in order to balance membrane recycling at low rates of stimulation, athough it can occur concomitantly with other modes of endocytosis (Neef et al., 2014). Besides the conventional molecular players 
-clathrin and dynamin-, a complex of otoferlin with AP-2 and Myosin IV has also been suggested to participate in CME (Duncker et al., 2013). The third mode, bulk endocytosis, is proposed as one of the principal mechanisms for IHCs. This mode relies on the formation of large endocytic cisternae in the vicinity of the ribbon when exocytosis exceeds 3-4 times the RRP equivalent (Kamin et al., 2014; Neef et al., 2014; Revelo et al., 2014). The cisternae are then further turned into smaller compartments, seemingly synaptic vesicles, in that they co-localize with synaptic vesicle markers and seem able to participate in another round of exocytosis (Kamin et al., 2014; Revelo et al., 2014). As this type of endocytosis is not commonly found in resting conditions (Neef et al., 2014), it seems as a clathrin- and dynamin- independent mechanism to retrieve big portions of membrane faster (time constant of $\sim 6 \mathrm{~s}$ ) than CME. Thus, bulk endocytosis seems to mediate active zone clearance to counteract the sustained high rate of exocytosis observed in ribbon synapses (Holt et al., 2003; Paillart et al., 2003).

However, considering the rapid vesicle pool resupply (with a maximum rate of $\sim 1200-1900$ vesicle/s in IHC, (Griesinger et al., 2005; Moser and Beutner, 2000)), CME and bulk endocytosis seem too slow to account for RRP recovery. The large reserve pool of synaptic vesicles observed in IHCs might play an important role in the replenishment of the ribbon (Kamin et al., 2014). Therefore, it appears that endocytosis is rather involved in the resupply of the RRP at longer timescales (Moser and Beutner, 2000).

Importantly, intracellular $\mathrm{Ca}^{2+}$ concentration seems to act as a switch between the different modes of endocytosis in IHCs and determine the onset and extent of each mode. The ocurrance of rapid endocytosis increases from $15 \%$ to $90 \%$ when intracellular $\mathrm{Ca}^{2+}$ concentration passes from $15 \mu \mathrm{M}$ to $40 \mu \mathrm{M}$ (Beutner et al., 2001). Lower $\mathrm{Ca}^{2+}$ concentrations trigger a slower endocytic retrieval, probably reflecting CME (Beutner et al., 2001; Neef et al., 2014). Interestingly, the kinetics of these two modes of endocytosis remain unaffected by changes in intracellular $\mathrm{Ca}^{2+}$ levels (Beutner et al., 2001; Moser and Beutner, 2000; Neef et al., 2014). Whether this holds true for bulk endocytosis remains to be tested.

The described $\mathrm{Ca}^{2+}$ dependence of endocytosis suggests a tight coupling between exo- and endocytosis in IHCs. In this context, the presumable $\mathrm{Ca}^{2+}$-sensor, otoferlin, might couple the two processes via interaction with adaptor proteins implicated in endocytosis per se and active zone clearance (Duncker et al., 2013; Jung et al., 2015; Neef et al., 2014; Revelo et al., 2014). 


\section{Calcium channels at IHCs}

Transmitter release at the IHC is driven by the influx of $\mathrm{Ca}^{2+}$ through voltage gated calcium channels (Brandt et al., 2003; Dou et al., 2004; Platzer et al., 2000). They belong to the Ltype class of dihydropyridine sensitive $\mathrm{Ca}^{2+}$ channels which are encoded by the $\mathrm{Ca}_{\mathrm{v}} 1.3 \alpha_{1} \mathrm{D}$ gene. Overall, these type of $\mathrm{Ca}^{2+}$ channels are well suited to respond to graded potentials. Their rapid gating ( $\tau$ of activation of $300 \mu \mathrm{s}$ at room temperature) ensures short delays in synaptic transmission (Xu and Lipscombe, 2001) and supports the proper response to voltage fluctuations of the periodic (AC) and sustained (DC) component of the receptor potential. Their lower voltage of activation (between -65 and $-55 \mathrm{mV}$ ) compared to other members of the same class (Beutner et al., 2001; Koschak et al., 2001; Platzer et al., 2000; Spassova et al., 2004; $\mathrm{Xu}$ and Lipscombe, 2001), makes $\mathrm{Ca}_{\mathrm{v}} 1.3 \alpha_{1}$ channels crucial to mediate IHC responses to small membrane depolarizations and consequently, to transmit information from low sound intensities (Xu and Lipscombe, 2001). Additionally, their slow inactivation (Fuchs et al., 1990; Koschak et al., 2001; Roberts et al., 1990; Spassova et al., 2004) bolsters sustained signaling while eventually avoiding $\mathrm{Ca}^{2+}$ overload of IHCs (Johnson et al., 2008).

Several factors influence the spatiotemporal contribution of $\mathrm{Ca}^{2+}$ during IHC stimulation, including the topology of $\mathrm{Ca}^{2+}$ channels and the distribution of $\mathrm{Ca}^{2+}$ buffers. Although little is known about the precise distribution of $\mathrm{Ca}^{2+}$ buffers at IHCs active zones, the topology of $\mathrm{Ca}^{2+}$ channels have been intensively studied. $\mathrm{Ca}^{2+}$ channels are abundantly expressed in the basolateral surface of the IHCs (Brandt et al., 2005). In mature IHCs, most of the $\mathrm{Ca}^{2+}$ channels are clustered as a stripe under the synaptic ribbon as shown by super-resolution immunofluorescence and electron microscopy (Neef et al., 2018; Wong et al., 2014). Electrophysiological measurements of macroscopic and single-channel currents have led to an estimation of 1000-3000 $\mathrm{Ca}^{2+}$ channels per IHC (Brandt et al., 2005; Graydon et al., 2011; Zampini et al., 2010), with less than $10 \%$ of them located extrasynaptically after the onset of hearing (Wong et al., 2014). Thus, based on the number of synapses per IHC, it was initially suggested that each active zone harbors 40-180 channels. Recently, a study combining confocal microscopy, optical fluctuation analysis and 3D-STED microscopy set the estimation between $30-360 \mathrm{Ca}^{2+}$ channels at each active zone (Neef et al., 2018).

In addition to differences in the number of $\mathrm{Ca}^{2+}$ channels per active zone, the coupling between the $\mathrm{Ca}^{2+}$ source and the $\mathrm{Ca}^{2+}$ sensor critically determines how the acoustic stimulus is encoded at the synapse between the IHC and the SGN. However, morphological studies 
using freeze-fracture to determine the arrangement of $\mathrm{Ca}^{2+}$ channels are difficult to achieve. This comes in addition to the limited knowledge regarding the number of functional $\mathrm{Ca}^{2+}$ channels that open at different sound intensities. Therefore, indirect techniques are used to estimate the $\mathrm{Ca}^{2+}$ channel cooperativity (i.e., the number of $\mathrm{Ca}^{2+}$ channels contributing to vesicle fusion). A classical approach is to relate the presynaptic $\mathrm{Ca}^{2+}$ influx and exocytosis during an experimental reduction in the number of active $\mathrm{Ca}^{2+}$ channels or in the single channel current (Augustine et al., 1991; Eggermann et al., 2012). The change in the number of active $\mathrm{Ca}^{2+}$ channels can be achieved either by gradually changing the strength of depolarization, or by slowly applying $\mathrm{Ca}^{2+}$ channel blockers. The obtained apparent $\mathrm{Ca}^{2+}$ cooperativity reflects the active zone morphology. Instead, if the single $\mathrm{Ca}^{2+}$ channel current is experimentally varied, the resulting relationship between $\mathrm{Ca}^{2+}$ influx and exocytosis will reflect the intrinsic cooperativity (i.e., number of $\mathrm{Ca}^{2+}$ binding steps required for one vesicle to fuse) [for a review see (Eggermann et al., 2012)].

There are two opposing hypotheses regarding the $\mathrm{Ca}^{2+}$ control of vesicular release. The $\mathrm{Ca}^{2+}$ nanodomain hypothesis of exocytosis control proposes that one or very few $\mathrm{Ca}^{2+}$ channels in nanometer proximity (less than $100 \mathrm{~nm}$ ) from the vesicular release site govern the $\mathrm{Ca}^{2+}$ concentration that drives the release of a synaptic vesicle (Moser et al., 2006a; Neher, 1998). Exocytosis is thus linearly dependent on the number of open $\mathrm{Ca}^{2+}$ channels, leading to an apparent cooperativity close to 1 . This results in increased sensitivity and speed of synaptic transmission, and a better adaptation for encoding graded potentials with a large dynamic range (Eggermann et al., 2012; Jarsky et al., 2010; Matveev et al., 2011). Contrary, the $\mathrm{Ca}^{2+}$ microdomain hypothesis proposes that a pool of several $\mathrm{Ca}^{2+}$ channels located further away from the vesicular $\mathrm{Ca}^{2+}$ sensor $(>100 \mathrm{mn})$ are necessary to elicit exocytosis of a given vesicle [for a review see (Eggermann et al., 2012; Oheim et al., 2006)]. In this case, exocytosis shows a nonlinear dependence on the number of open channels (i.e., high apparent $\mathrm{Ca}^{2+}$ cooperativity close or equal to the intrinsic $\mathrm{Ca}^{2+}$ cooperativity). Stochastic $\mathrm{Ca}^{2+}$ channel openings have then a reduced impact in the exocytosis of vesicles and therefore, synaptic transmission is predicted to have less jitter and noise (Matveev et al., 2009, 2011).

Fusion of vesicles in IHCs exhibits a high intrinsic $\mathrm{Ca}^{2+}$ cooperativity of 4-5 determined using flash photolysis of caged $\mathrm{Ca}^{2+}$ (Beutner et al., 2001). This implies that at least $4 \mathrm{Ca}^{2+}$ ions have to bind the $\mathrm{Ca}^{2+}$ sensor in a cooperative manner. The non-linearity in the intrinsic dependence of release means that synaptic transmission strongly depends on the distance of the vesicle to the $\mathrm{Ca}^{2+}$ channel. This distance has been estimated from the effects of EGTA, 
a slow $\mathrm{Ca}^{2+}$ chelator, on vesicle fusion. Supporting the $\mathrm{Ca}^{2+}$ nanodomain hypothesis, EGTA has little effect on exocytosis from apical IHCs (Moser and Beutner, 2000). Taking into account the rate of diffusion of $\mathrm{Ca}^{2+}$ and the binding kinetics of EGTA to $\mathrm{Ca}^{2+}$, the weighted average coupling distance between a $\mathrm{Ca}^{2+}$ channel and the release site has been estimated around 15-17 nm (Pangršič et al., 2015).

The variety and distribution of $\mathrm{Ca}^{2+}$ buffers in the cell also shape the cytoplasmic gradient of $\mathrm{Ca}^{2+}$ away from the $\mathrm{Ca}^{2+}$ source. These buffers can be mobile or fixed, including $\mathrm{Ca}^{2+}-$ channel interacting proteins. Diffusion of $\mathrm{Ca}^{2+}$ is increased by mobile buffers, yet slowed by fixed buffers (Zhou and Neher, 1993). The major mobile $\mathrm{Ca}^{2+}$-binding proteins in IHCs are the EF-hand $\mathrm{Ca}^{2+}$ mobile buffers such as calretinin, parvalbumin- $\alpha$ and calbindin-D28k (and oncomodulin that is only present transiently during development, Hackney et al., 2005; Pangršič et al., 2015). Their absence enhances $\mathrm{Ca}^{2+}$ dependent inactivation, while the $\mathrm{Ca}^{2+}$ channel activation kinetics remain intact (Pangršič et al., 2015). It is not clear yet whether these buffers interact directly with the $\mathrm{Ca}^{2+}$ channel, but the direct interaction of calretinin with $\mathrm{Ca}_{\mathrm{v}} 2.1$ channels opens the possibility (Christel et al., 2012).

$\mathrm{Ca}_{\mathrm{v}} 1.3$ channels have several reported interacting partners in IHC. These interacting proteins often modify the biophysical properties and behaviors of the $\mathrm{Ca}^{2+}$ channels, and are involved in $\mathrm{Ca}^{2+}$ signaling. Among the most prominent ones is the $\mathrm{Ca}^{2+}$ sensor Calmodulin $(\mathrm{CaM})$. $\mathrm{CaM}$ associates with the $\mathrm{COOH}$-terminal domain of L-type $\mathrm{Ca}^{2+}$ channels (Ben Johny et al., 2013). Upon $\mathrm{Ca}^{2+}$ influx, $\mathrm{Ca}^{2+}$ binds to this preassociated complex and triggers a conformational change that mediates $\mathrm{Ca}^{2+}$ dependent inactivation. Calcium binding proteins (CaBPs) are also a crucial family of proteins that interact with $\mathrm{Ca}^{2+}$ channels. Upon $\mathrm{Ca}^{2+}$ binding, they appear to displace $\mathrm{CaM}$ from the $\mathrm{Ca}^{2+}$ channel antagonizing the $\mathrm{CaM}$ dependent inactivation (Hardie and Lee, 2016). CaBP2 disruption is associated to a significant hearing impair in humans (Picher et al., 2017a; Schrauwen et al., 2012), while CaBP4 mildly affects IHC physiology (Cui et al., 2007), which argues for a potential functional redundancy among the CaBP family members.

\section{Spiral ganglion neurons and their in vivo response properties}

As mentioned before, SGNs connect the hair cells to the central auditory pathway. Two types of SGNs are present in the cochlea: type I and type II. Type I SGNs constitute the vast majority (90-95\%) of cochlear nerve afferents (Liberman, 1982; Spoendlin, 1969). They receive input solely from IHCs (Robertson, 1984) and are myelinated almost in all their 
length with the exception of a small segment close to the postsynaptic bouton. Type II SGNs, the remaining $5-10 \%$, form multiple synapses with several OHCs. Contrary to type I, type II SGNs are unmyelinated and do not seem to be involved in sound encoding but rather in monitoring the state of the organ of Corti (Spoendlin, 1969, 1972). Type II afferents will not be considered further in this section.

The central axon of the SGNs projects to the brainstem forming the eight cranial nerve. In vivo recordings from ANFs have been crucial to characterize SGNs and to understand the first synapse of the auditory system. ANFs fire action potentials spontaneously in the absence of sound. In mammals, the spontaneous firing rate (SR) ranges from close to zero spikes/s to more than 150 spikes/s (Kiang et al., 1965; Tsuji and Liberman, 1997). The SR can be used to classify ANFs in 3 groups (Liberman, 1982): i) high SR with $>18$ spikes/s, ii) medium with $0.5<\mathrm{SR} \leq 18 \mathrm{spikes} / \mathrm{s}$, and iii) low $\mathrm{SR}$ with $\leq 0.5 \mathrm{spikes} / \mathrm{s}$. Those 3 groups have been associated with a distribution along the pillar/modiolar axis of the IHC (Liberman, 1980; Merchan-Perez and Liberman, 1996; Tsuji and Liberman, 1997). In mice, ANFs are classified only in high $(\geq 1 \mathrm{EPSC} / \mathrm{s})$ and low $(<1 \mathrm{EPSC} / \mathrm{s})$ SR fibers (Taberner and Liberman, 2005). Intracellular recordings and labeling of cat ANFs, in combination with electron microscopy, showed that high SR fibers have larger axon diameters and contact the IHC predominantly on the pillar side into AZs with smaller ribbons (Liberman, 1980). Low and medium SR fibers, on the other hand, have smaller axon diameters and form synapses with larger ribbons on the modiolar face of the IHC (Liberman et al., 2011). |Figure 2|

Several parameters have been used to describe the diversity of the synaptic response of ANFs. The threshold, for instance, dictates the sound pressure level required to increase the ANF response above a criterion (experimenters often use a 20 spikes/s increase in the ANF response above the SR, Johnson, 1980; Rose et al., 1967, 1971). The threshold as a function of the frequency is measured by the tuning curve, which is independent for each ANF and presents a V-shape (Kiang et al., 1962). The characteristic frequency (CF) of an ANF is the frequency for which the ANF has the lowest threshold and corresponds to the tip of the tuning curve. This CF depends on the fiber's position on the basilar membrane (Liberman, 1980; Merchan-Perez and Liberman, 1996; Müller et al., 2005; Tsuji and Liberman, 1997). A physiological consequence of the classification based on the SR is that high SR fibers tipically have the lowest threshold, and medium/low SR the higuest one. This negative correlation between SR and threshold has been observed in mice (Taberner and Liberman, 
2005), cats (Liberman, 1980; Tsuji and Liberman, 1997), guinea pigs (Winter et al., 1990), gerbils (Huet et al., 2016; Ohlemiller et al., 1991) and ferret (Sumner and Palmer, 2012).

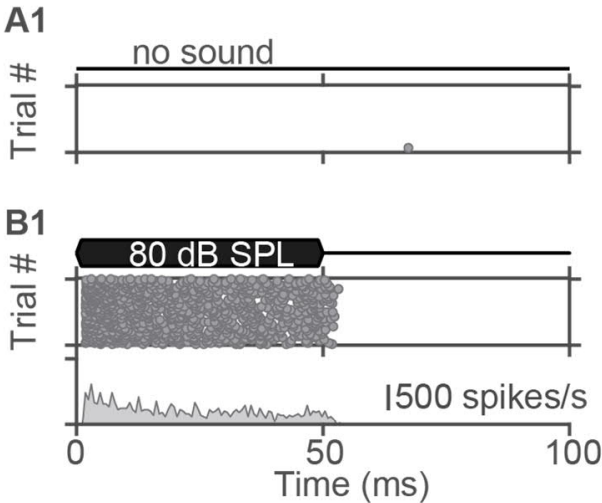

C1

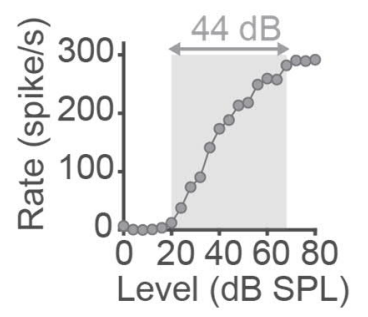

A2

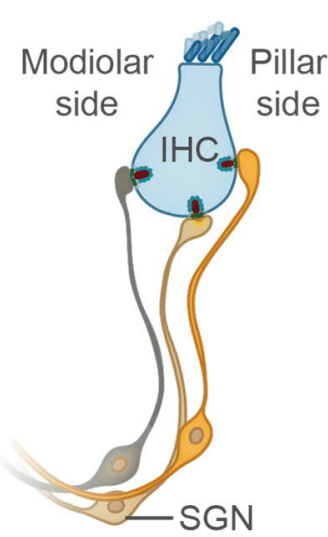

C2
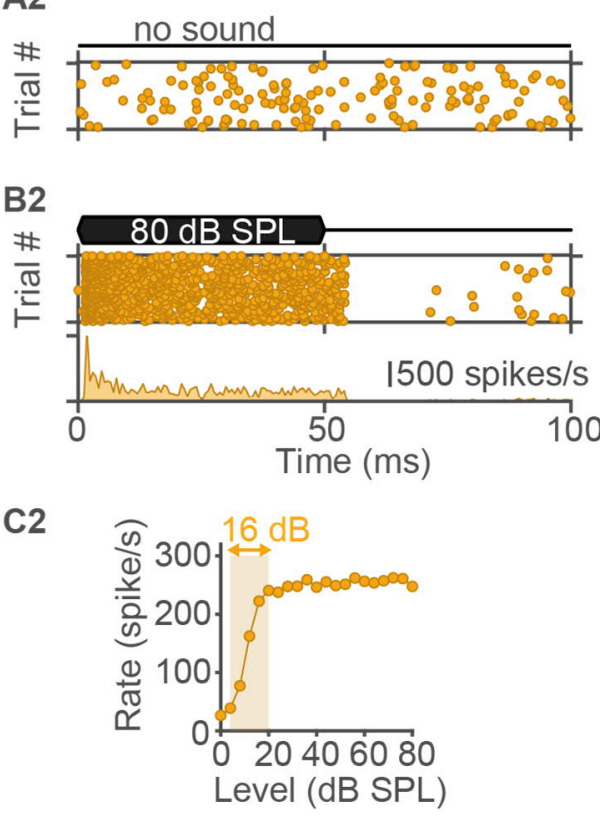

|Figure 2| In vivo response properties of spiral ganglion neurons

In vivo juxtacellular recordings from individual auditory nerve fibers have unraveled the diversity in their response properties. A. Spontaneous rate: Some ANFs (A1) have low spontaneous activity (low SR $<1$ spike/s, measured in absence of sound in mice), while some other ANFs (A2, upper panel) have high rates (high SR $\geq$ 1 spikes/s). (Taberner and Liberman, 2005) B. Evoked activity: In response to a sound stimulation, the low SR ANFs (B1) typically show a lower peak to adapted rate ratio than high SR ANFs (B2). It is thought that low SR fibers innervate prerefentially the IHC modiolar side, while the high SR innervate the pillar side. C. Intensity coding: Low SR ANFs typically have higher sound thresholds and a broader dynamic range (C1, shaded area). High SR ANFs show a low sound threshold and a narrow dynamic range (C2, shaded area) (Winter et al., 1990). Plots are courtesy of Dr. Antoine Huet.

The strength of the synaptic response has been addressed by studying the response dependence on the stimulus intensity. ANFs increase their AP rates from minimum to maximum over a range of dBSPL (dB Sound Pressure Level, $\mathrm{P}_{\mathrm{ref}}=10 \mu \mathrm{Pa}$ at $1 \mathrm{kHz}$, Ohlemiller and Echteler, 1990; Sachs et al., 1989; Winter et al., 1990, Ohlemiller et al., 1991). The resulting rate level function describes an ANF firing rate as stimulus intensity and allows to calculate the fiber's operating range (called dynamic range). The dynamic range is defined as the sound intensities for which the firing rate increases from $10 \%$ to $90 \%$ of the difference between spontaneous and maximal rates. Most murine ANFs have dynamic ranges of less than $15 \mathrm{~dB}$, yet there is a correlation between SR and dynamic range. High SR fibers increase their spiking rate steeply, leading to an early saturation of the response, and a narrow dynamic range (Ohlemiller et al., 1991; Taberner and Liberman, 2005; Winter 
et al., 1990). Contrary, low SR fibers display shallower rise in the spiking rate, with little or no saturation, thereby, a wider dynamic range. |Figure 2|

The fact that fibers with the same CF can have high or low SR supports the idea that a single IHC encompasses synapses of both types. How does a presumably isopotential and uncompartmentalized IHC harbor synapses with heterogeneous response properties? Recent studies indicate that part of the diversity in the afferent response originates from heterogeneous $\mathrm{Ca}^{2+}$ clusters within individual IHCs (Frank et al., 2009; Jean et al., 2019; Neef et al., 2018; Ohn et al., 2016). Postsynaptic mechanisms have also been postulated, including gradient in the number and/or properties of AMPA receptors (Liberman et al., 2011; Zhang et al., 2018), fiber diameter and mitochondrial enrichment (Merchan-Perez and Liberman, 1996) and varying levels of voltage-gated channels and $\mathrm{Ca}^{2+}$ mobile buffers (Petitpré et al., 2018; Shrestha et al., 2018; Sun et al., 2018). All these presynaptic and postsynaptic mechanisms most likely ensure the correct encoding of intensity information of the sound stimuli.

\section{Aim of this work}

The mechanisms that shape the impressive performance and heterogeneity of the IHC ribbon synapses are still scarcely understood. The first part of this work aims at understanding synaptic transmission at individual active zones. Here, I used paired IHC and bouton patchclamp recordings in near physiological conditions to investigate spontaneous activity, vesicle pool dynamics, stimulus-intensity coding and $\mathrm{Ca}^{2+}$ dependence of neurotransmitter release. The second part is focused on assessing the role of the synaptic ribbon on sound encoding using a RIBEYE KO mouse model. In collaboration with other researchers, we characterized the morphology and physiology of ribbonless active zones using immunofluorescence, electron microscopy, patch-clamp recordings, $\mathrm{Ca}^{2+}$ imaging and in vivo ANFs recordings. The last part of this work aims to determine the effect of the deletion of endophilin A in IHCs. Together with collaborators, we used single-cell RT and immunoblot to corroborate the expression of endophilin A. We further investigated exocytosis, endocytosis and the general morphology using immunofluorescence, electron microscopy, patch-clamp recordings and co-immunoprecipitations. 


\section{Chapter 2 Biophysical properties of inner hair cell ribbon synapses at near physiological conditions}

Lina M. Jaime Tobón ${ }^{1,2,3,4,5}$ and Tobias Moser ${ }^{1,2,3,4 \#}$

${ }^{1}$ Synaptic Nanophysiology Group, Max Planck Institute for Biophysical Chemistry, Göttingen, Germany

${ }^{2}$ Institute for Auditory Neuroscience and InnerEarLab, University Medical Center Göttingen, Göttingen, Germany

${ }^{3}$ Auditory Neuroscience Group, Max Planck Institute for Experimental Medicine, Göttingen, Germany

${ }^{4}$ Collaborative Research Center 889, University of Göttingen, Göttingen, Germany

${ }^{5}$ Göttingen Graduate School for Neuroscience and Molecular Biosciences, University of

Göttingen, Göttingen, Germany

${ }^{\#}$ corresponding authors

\section{Corresponding authors:}

Tobias Moser

Institute for Auditory Neuroscience and InnerEarLab, University Medical Center Göttingen, Göttingen, Germany

tmoser@gwdg.de

Tel.: +49-551-39-63070

Fax: $+49-551-3912950$

Detailed author contribution of L. M. Jaime Tobón

- Experimental work and analysis:

- Performed and analyzed paired recordings

- Prepared the first manuscript draft. 


\section{Introduction}

Inner hair cells (IHCs) convey the acoustic information to higher stages of the auditory system through their synapses with type I spiral ganglion neurons (SGNs). Upon mechanoelectrical transduction, the IHC receptor potential triggers the opening of L-type voltage-gated $\mathrm{Ca}^{2+}$ channels, mediating the fusion of vesicles with the plasma membrane (Brandt et al., 2003; Moser and Beutner, 2000; Platzer et al., 2000; Spassova et al., 2001). Glutamate released from the presynaptic active zone binds AMPA receptors located in the postsynaptic bouton (Glowatzki and Fuchs, 2002; Keen and Hudspeth, 2006; Matsubara et al., 1996; Schnee et al., 2013), ultimately initiating an action potential in the SGN (Rutherford et al., 2012). Sound encoding is thought to rely on individual IHC active zones providing the sole input to individual SGNs [for review, see (Fettiplace, 2017; Moser and Vogl, 2016)].

In vivo juxtacellular recordings from auditory nerve fibers (ANFs) have unraveled key physiological aspects of synaptic transmission. First, release occurs even in the absence of sound at rates greater than 100 spikes/s, depending on the species (Barbary, 1991; Evans, 1972; Kiang et al., 1965; Schmiedt, 1989; Taberner and Liberman, 2005). Second, strong sound elicits onset responses of approximately one thousand spikes per second within submilliseconds (Taberner and Liberman, 2005). During continuous stimulation, the initial firing rate adapts to hundreds of spikes per second, yet release continues indefatigably as long as the sound lasts (Westerman and Smith, 1984). Third, SGNs differ in their response to increasing tone levels. The resulting rate level functions vary within SGNs. Some fibers show a low sound threshold and a steep increase in the spike rate to increasing sound intensities until the rate saturates, while other fibers have a higher sound threshold and shallower spike rate increment with saturation at higher sound intensities (Winter et al., 1990). Furthermore, there is a relation of the latter characteristics to the spontaneous rate of the fibers. Low threshold fibers have a high spontaneous firing rate, while high threshold fibers have a low spontaneous firing rate (Ohlemiller et al., 1991). |Figure 2|

Differences in the response properties have been attributed to both presynaptic and postsynaptic features, including presynaptic heterogeneity and diverse postsynaptic molecular composition [for a comprehensive overview, see (Meyer and Moser, 2010; Moser et al., 2019; Rutherford and Moser, 2016)]. Despite efforts to dissect the underlying mechanisms of these response properties, the prevalent techniques only provide information 
from either one of the synaptic players, IHC or SGN, limiting the conclusions. Our understanding of sound encoding could be much advanced by biophysical studies of transmitter release from single IHC active zones of hearing animals performed under near physiological conditions. This approach provides detailed information on synaptic transmission for the interpretation of in vivo recordings of spontaneous and sound-evoked firing of SGNs.

Here we studied the biophysical properties of the auditory ribbon synapse using paired recordings from IHC and postsynaptic boutons in near physiological conditions in organs of Corti from hearing mice. This technique allows a tight control of the presynapse while recording the postsynaptic response of a single active zone. Our results emphasize that murine IHC synapses have differences in the spontaneous rate that depends on the topographical position of the synapses (modiolar or pillar). High SR synapses displayed spontaneous EPSCs of higher amplitude and more compact waveform, lower threshold and a tendency to shorter latencies of evoked neurotransmitter release, as well as faster recovery from RRP depletion. Our study also provides strong and direct support that a $\mathrm{Ca}^{2+}$ nanodomain-like control of synaptic vesicle fusion operates during physiological sound encoding.

\section{Materials and Methods}

\section{Animals and tissue preparation}

c57BL/6N mice of either sex between postnatal day 14-23 (p14-23) were used. The animal handling and experiments complied with national animal care guidelines and were approved by the University of Göttingen Board for animal welfare and the Animal Welfare Office of the State of Lower Saxony. Animals were sacrificed by decapitation and the cochleae were extracted in modified Hepes Hank's solution containing: $5.36 \mathrm{mM} \mathrm{KCl}, 141.7 \mathrm{mM} \mathrm{NaCl}, 1$ $\mathrm{mM} \mathrm{MgCl} 2-6 \mathrm{H}_{2} \mathrm{O}, 0.5 \mathrm{mM} \mathrm{MgSO} 4-7 \mathrm{H}_{2} \mathrm{O}, 10 \mathrm{mM}$ HEPES, $0.5 \mathrm{mg} / \mathrm{ml}$ L-glutamine, and 1 $\mathrm{mg} / \mathrm{ml} \mathrm{D-glucose}$ ( $\mathrm{pH} \mathrm{7.2,} \mathrm{osmolarity} \mathrm{of} \sim 300 \mathrm{mOsm}$ ). The apical coil of the organ of Corti was dissected and placed under a grid in the recording chamber. Pillar or modiolar supporting cells were removed using soda glass pipettes in order to gain access to the basolateral face of the IHCs and to the postsynaptic boutons of type I SGNs. Dissection of the organ of Corti and cleaning of the supporting cells were performed at room temperature $\left(20-25^{\circ} \mathrm{C}\right)$. 


\section{Electrophysiological recordings}
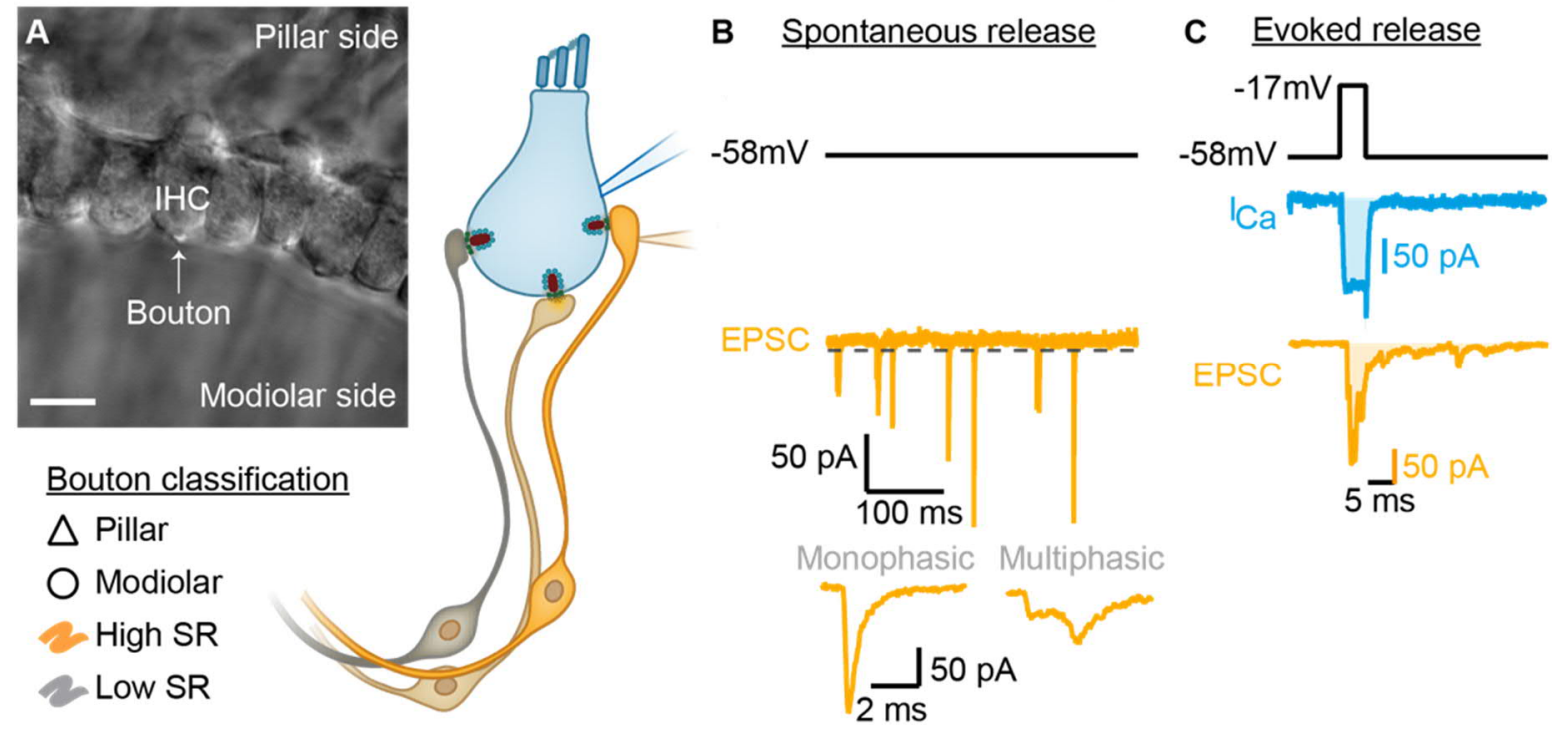

|Figure 3| Paired IHC-bouton patch-clamp recordings to study the biophysical properties of individual IHC ribbon synapses

A. Differential Interference Contrast (DIC) image of an explanted mouse organ of Corti. Supporting cells from the modiolar side were removed to gain access to the IHCs and their contacting boutons. The recorded boutons were classified based on their position ( $\triangle$ pillar or $\mathrm{O}$ modiolar) and on their spontaneous activity (Low $\mathrm{SR}<$ $1 \mathrm{EPSC} / \mathrm{s}$ vs High SR $\geq 1 \mathrm{EPSC} / \mathrm{s})$. Scale bar: $10 \mu \mathrm{m}$. B. Spontaneous release was recorded in absence of stimulation (i.e., IHC holding potential $=-58 \mathrm{mV}$ ). EPSCs were classified as monophasic (a steady rising and monoexponential decay) or as multiphasic (multiple inflections and slowed raising and decaying kinetics). $\mathbf{C}$. Evoked release was recorded using depolarizing pulses (black trace), triggering whole $\mathrm{IHC} \mathrm{Ca}^{2+}$ influx $\left(\mathrm{I}_{\mathrm{Ca}}\right.$, blue trace) and concomitant release of neurotransmitter (EPSC, light orange trace). $\mathrm{Ca}^{2+}$ charge and EPSC charge were estimated by taking the integral of the current (shaded light blue and light orange areas).

Pre- and postsynaptic paired patch clamp recordings were performed at near physiological temperature $\left(32-37^{\circ} \mathrm{C}\right)$ using an EPC-9 amplifier (HEKA electronics). |Figure 3| Patch electrodes were positioned using a PatchStar micromanipulator (Scientifica, UK). Wholecell recordings from IHCs were achieved using the perforated-patch clamp technique (Moser and Beutner, 2000) using Sylgard ${ }^{\mathrm{TM}}$-coated $1.5 \mathrm{~mm}$ borosilicate pipettes with typical resistances between 3.5 and $6 \mathrm{M} \Omega$. The IHC pipette solution contained: $129 \mathrm{mM} \mathrm{Cs}-$ gluconate, $10 \mathrm{mM}$ tetraethylammonium (TEA)-Cl, $10 \mathrm{mM}$ 4-AP, $10 \mathrm{mM}$ HEPES, $1 \mathrm{mM}$ $\mathrm{MgCl}_{2}$ (pH 7.2, osmolarity of $\sim 290 \mathrm{mOsm}$ ), as well as $300 \mu \mathrm{g} / \mathrm{ml}$ amphotericin B added prior to the experiment. Once the series resistance of the IHC reached below $30 \mathrm{M} \Omega$, whole-cell voltage-clamp recordings from a contacting bouton was performed largely as described in previous studies (Glowatzki and Fuchs, 2002; Grant et al., 2010; Huang and Moser, 2018). 


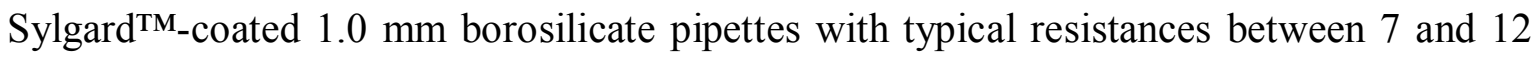
$\mathrm{M} \Omega$ were used for the postsynaptic recordings. The bouton pipette solution contained: 137 mM KCl, 5 mM EGTA, 5 mM HEPES, 1 Mm Na2-GTP, $2.5 \mathrm{mM} \mathrm{Na}$-ATP, $3.5 \mathrm{mM}$ $\mathrm{MgCl}_{2} \cdot 6 \mathrm{H}_{2} \mathrm{O}$ and $0.1 \mathrm{mM} \mathrm{CaCl}_{2}(\mathrm{pH} 7.2$ and osmolarity of $\sim 290 \mathrm{mOsm})$. The organ of Corti was continuously perfused with an extracellular solution containing $4.2 \mathrm{mM} \mathrm{KCl}, 95-100$ $\mathrm{mM} \mathrm{NaCl}, 25 \mathrm{mM} \mathrm{NaHCO} 3,30 \mathrm{mM}$ TEA-Cl, $1 \mathrm{mM}$ Na-Pyruvate, $0.7 \mathrm{mM} \mathrm{NH} \mathrm{PO}_{4} \cdot \mathrm{H}_{2} \mathrm{O}$, $1 \mathrm{mM} \mathrm{CsCl}, 1 \mathrm{mM} \mathrm{MgCl}{ }_{2} \cdot \mathrm{H}_{2} \mathrm{O}, 1.3 \mathrm{mM} \mathrm{CaCl}_{2}$, and $11.1 \mathrm{mM}$ D-glucose (pH 7.3, osmolarity of $\sim 310 \mathrm{mOsm}$ ). $2.5 \mu \mathrm{M}$ tetrodotoxin (Tocris or Santa Cruz) was added to block voltagegated $\mathrm{Na}^{+}$channels in the postsynaptic bouton.

Data were acquired using the Patchmaster software (HEKA electronics). The current signal was sampled at $20-50 \mathrm{kHz}$ and filtered at $5-10 \mathrm{kHz}$. IHC were voltage-clamped at a holding potential of $-58 \mathrm{mV}$, corresponding to the presumable in vivo resting potential (Johnson, 2015). The bouton was hold at a potential of $-94 \mathrm{mV}$. All reported potentials are corrected by the liquid junction potential ( $19 \mathrm{mV}$ for the IHC and $4 \mathrm{mV}$ for the bouton). $\mathrm{Ca}^{2+}$ current recordings were corrected for the linear leak current using a $P / n$ protocol. We excluded IHCs and boutons with leak currents exceeding $-60 \mathrm{pA}$ and $-100 \mathrm{pA}$ at holding potential, respectively. The series resistance of the IHCs was typically below $30 \mathrm{M} \Omega$. The apparent series resistance of the bouton was calculated from the capacitive transient in response to a $10-\mathrm{mV}$ test pulse. The actual series resistance $\left(\mathrm{R}_{\mathrm{s}}\right)$ was offline calculated as reported in (Huang and Moser, 2018). Recordings with bouton Rs > $70 \mathrm{M} \Omega$ were discarded.

Spontaneous activity was calculated from time windows without stimulation when the IHC was held at the resting potential (see above); either from a $5-10 \mathrm{~s}$ recording or by averaging the number of events from the segments preceding a depolarizing pulse. |Figure $3 \mathrm{~B} \mid$ To study the depletion and recovery of the pool of vesicles, we used a protocol adapted from the forward masking protocol performed during in vivo extracellular recordings of SGNs (Harris and Dallos, 1979; Jean et al., 2018). It consisted of two consecutive depolarizing pulses to the voltage that elicited the highest peak of $\mathrm{Ca}^{2+}$ current $(-17 \mathrm{mV})$. $\mid$ Figure $3 \mathrm{C} \mid$ The first pulse, called masker, lasted $100 \mathrm{~ms}$ and it was followed by a second pulse, called probe, which lasted $15 \mathrm{~ms}$. The two pulses were separated by intervals without depolarization (interstimulus intervals, ISI) that lasted 4, 16, 64 and $256 \mathrm{~ms}$. The waiting time between masker and masker was $20 \mathrm{~s}$ and each protocol was repeated between $3-20$ times. To study dynamic range, we used a current-voltage (IV) protocol with $10 \mathrm{~ms}$ pulses of increasing voltage (from $-70 \mathrm{mV} /-60 \mathrm{mV}$ to $70 \mathrm{mV}$ in $5 \mathrm{mV}$ steps). The interval between two stimuli 
was $1.5 \mathrm{~ms}$. The apparent $\mathrm{Ca}^{2+}$ dependence of neurotransmitter release was studied using 2 to $5 \mathrm{~ms}$ step-depolarizations, and using different intensities of depolarization or the slow perfusion of $\mathrm{Ca}^{2+}$ channel blockers to vary the $\mathrm{Ca}^{2+}$ influx into the IHC. For the latter, isradipine (Sigma-Aldrich) or $\mathrm{ZnCl}_{2}$ (Sigma-Aldrich) were added to the extracellular solution and slowly perfused into the chamber while recording the responses to a $5 \mathrm{~ms}$ pulse. Isradipine was diluted to a final concentration of $0.5-2 \mu \mathrm{M}$ from a stock of $20 \mathrm{mM}$ in DMSO. $\mathrm{ZnCl}_{2}$ was diluted to a final concentration of $1 \mathrm{mM}$ from a stock of $0.1 \mathrm{M}$ and filtered by 0.2 $\mu \mathrm{m}$. The time interval between two subsequent depolarizations was $20 \mathrm{~s}$. In addition, we used a tail current protocol to study release in response to graded numbers of open $\mathrm{Ca}^{2+}$ channels.

\section{Data Analysis}

Electrophysiological data was analyzed using the IgorPro 6 Software Package (Wavemetrics), GraphPad Prism and Excel. $\mathrm{Ca}^{2+}\left(\mathrm{Q}_{\mathrm{Ca}}\right)$ and EPSC charge (QEPSC) were estimated by taking the integral of the current.

For forward masking experiments, the postsynaptic response was averaged for all the repetitions from each paired recording (between 3 and 20, depending on the stability of the pair). Single active zone pool dynamics were determined by fitting an exponential plus line function to the individual EPSC charge plots. RRP size (in vesicles) was estimated from the amplitude of the exponential function divided by the charge of the average sponEPSC for each pair. Sustained exocytosis rate (in vesicles per s) was calculated from the slope of the linear function divided the charge of the average sponEPSC. Individual recovery kinetics were determined from the ratio of probe and masker responses at $10 \mathrm{~ms}$ of the depolarization, with the ratio between masker and masker being 1 . The time when $50 \%$ of the response recovered was noted as half time of recovery.

To obtain IV curves, we averaged the evoked $\mathrm{Ca}^{2+}$-currents $\left(\mathrm{I}_{\mathrm{Ca}}\right)$ during 5 to $10 \mathrm{~ms}$ after the start of each depolarization. Fractional activation of the $\mathrm{Ca}^{2+}$ channels $\left(\mathrm{P}_{\text {activation }}\right)$ was obtained from the normalized chord conductance, $g$,

$$
g=\frac{I}{\left(V-V_{\text {rev }}\right)}
$$


where $\mathrm{V}$ is the membrane potential and $\mathrm{V}_{\text {rev }}$ is the reversal potential determined by fitting a line function between the voltage of $\mathrm{I}_{\text {Ca peak }}+10 \mathrm{mV}$ and the maximal depolarization. The activation curve was approximated by a first-order Boltzmann equation:

$$
g=\frac{g_{\max }}{1+\exp \left(\frac{V_{h c a}-V}{S}\right)}
$$

where $g_{\max }$ is the maximum chord conductance, $\mathrm{V}_{\mathrm{h} C a}$ is the membrane potential at which the conductance is half activated, and $S$ is the slope factor describing the voltage sensitivity of activation.

Rate level curves were obtained by calculating QEPSC by the end of each depolarization step and fitted using a sigmoidal function:

$$
R=R_{\text {spon }}+\frac{R_{\text {max }}}{1+\exp \left(\frac{V_{h E P S C}-V}{\text { rate }}\right)}
$$

where $R_{\text {spon }}$ is the spontaneous vesicle release, $R_{\max }$ is the maximal vesicle release, $V_{h}$ EPSC corresponds to the voltage of half-maximal release and $\mathrm{R}$ is the rate of release. The dynamic range was determined as the range of voltage between $10 \%$ and $90 \%$ of the maximal vesicle release.

$\mathrm{Ca}^{2+}$-dependence of release was determined by fitting the $\mathrm{QEPSC}_{\mathrm{ES}} \mathrm{Q}_{\mathrm{Ca}}$ plots with a power of exponent function:

$$
Q_{E P S C}=\mathrm{a}+\mathrm{b}\left(Q_{C a}\right)^{m}
$$

where $m$ corresponds to the $\mathrm{Ca}^{2+}$ cooperativity.

For dynamic range analysis, we included only pairs for which both the $\mathrm{Ca}^{2+}$ fractional activation and the rate level curves were possible to fit. Similarly, we only included pairs with effective fitting for analysis of the apparent $\mathrm{Ca}^{2+}$ dependence of release.

Data was prepared for presentation using Adobe Illustrator and Adobe InDesign. Skewness of the histograms from |Figure 4| was determined using GraphPad Prism. Statistical significance was assessed with unpaired $t$ test or a non-parametric Mann-Whitney test. Data is expressed as mean \pm sem. The box plots show $25^{\text {th }}, 50^{\text {th }}$ and $75^{\text {th }}$ percentiles with the individual data points overlaid. 


\section{Model of RRP release and replenishment}

We adapted a previously developed biophysical model of RRP pool dynamics (Buran et al., 2010; Frank et al., 2010; Jean et al., 2018; Jung et al., 2015). Briefly, the model assumes a fixed number of release sites $\left(\mathrm{N}_{\text {slots }}\right)$. RRP is composed by readily releasable vesicles that occupy these release sites. Each vesicle can fuse at any time point with a probability described by the fusion rate constant, $k_{\text {fusion. }}$ The refilling of an empty slot is described by the refill rate constant, $k_{\text {refill }}$. Both $k_{\text {fusion }}$ and $k_{\text {refill }}$ are stimulus-dependent, meaning they will pass from $k_{\text {fusion, spont }}$ and $k_{\text {refill, spont }}$ to $k_{\text {fusion, stim }}$ and $k_{\text {refill, stim }}$ with the depolarization of the IHC from resting potential to a potential eliciting the maximal $\mathrm{Ca}^{2+}$ influx. Originally, the model was developed to fit the poststimulus time histograms of SGNs in response to tone bursts. Therefore, we modified the model to fit EPSC recordings from the postsynaptic bouton by 1) excluding the factor $f$ that scales the fraction of exocytic events triggering an action potential and 2) removing refractoriness.

The model was fit to the grand average of the aligned responses for each forward masking interval $(4,16,64,256 \mathrm{~ms})$. To obtain a grand average that is not smeared out due to differences in synaptic delays between pairs, EPSC average traces were smoothed (3-point box smooth) and aligned by the point where the response was above $50 \%$ of the peak response for each pair. The resulting grand average was fit with the modified model waveform using a genetic fit algorithm implemented in IgorPro 6 (Wavemetrics). Importantly, only the first $50 \mathrm{~ms}$ of the synaptic response was included in the fit of the model. Later adaptation processes are not accounted by the model's equations and were therefore disregarded.

\section{Results}

In the present study, we analyzed synaptic release at individual active zones of murine IHCs after the onset of hearing. We used perforated patch-clamp recordings from the IHCs and simultaneous whole-cell rupture patch-clamp from the associated postsynaptic SGN bouton. |Figure 3| Our recordings were performed at body temperature and in artificial perilymphlike solution (Wangemann and Schacht, 1996) aiming to preserve the physiological resting conditions of the IHCs. 


\section{Spontaneous synaptic vesicle release}

In the absence of sound, SGN fire spontaneously as shown by in vivo recordings from single auditory nerve fibers (e.g. for mice: Taberner and Liberman, 2005). In our experimental conditions, spontaneous EPSCs (sponEPSC) |Figure 4A| were observed in 18 of 23 pairs in the absence of stimulation (i.e. when the IHC was held at $-58 \mathrm{mV}$, approximating its physiological resting potential (Johnson, 2015)). By holding the bouton at $-94 \mathrm{mV}$ and including TTX in the bath solution, we ensure that the recorded postsynaptic activity was not contaminated with voltage-gated $\mathrm{Na}^{+}$currents. Amplitudes of sponEPSC in all pairs typically varied from around $-10 \mathrm{pA}$ to $-400 \mathrm{pA} \mid$ Figure $4 \mathrm{~B} \mid$. One pair displaying exceptionally large sponEPSCs as big as $-800 \mathrm{pA}$ was excluded from further statistical analysis throughout, but it is displayed as an outlier. The amplitude histogram for all pairs was slightly skewed towards larger amplitudes (skewness of 1.15) with a coefficient of variation $(\mathrm{CV})$ of $0.73 \mid$ Figure $4 \mathrm{D} \mid$. The charge distribution for all pairs displayed a prominent peak between 45-60 fC, a skewness of 1.88 and a CV of 0.97 |Figure 4E|.

To establish the paired recording, we approached boutons facing either the pillar or the modiolar face of the IHC, allowing us to make a distinction between synapses from each side |Figure 3A|. Due to the technical difficulty of establishing the paired recording, typically only one bouton was recorded per IHC. Interestingly, the average amplitude of the sponEPSCs from modiolar boutons was significantly smaller than of pillar boutons (mean \pm s.e.m.: $-49.28 \pm 12.34$ pA vs $-94.75 \pm 6.92$ pA; $p=0.0104$, Mann-Whitney test) $\mid$ Figure $4 \mathrm{C} \mid$. However, the mean charge did not differ between the two sides $(54.61 \pm 13.46 \mathrm{fC}$ for modiolar boutons vs $76.62 \pm 7.78 \mathrm{fC}$ for pillar boutons; $p=0.2478$, Mann-Whitney test) |Figure $4 \mathrm{~F} \mid$. The striking difference in amplitude, yet not in charge, between both sides could result from slower sponEPSC kinetics in modiolar synapses. We therefore quantified the percentage of multiphasic sponEPSCs (i.e. EPSCs with inflections and slowed kinetics on their rising or decaying phases as initially reported by (Glowatzki and Fuchs, 2002)) for each synapse. Synapses from the modiolar side displayed on average $\sim 20 \%$ more multiphasic sponEPSCs ( $p=0.0969$, Mann-Whitney test) |Figure 4G|. 
A Pair \#1260618_1 Pair \#1090718_2 $\mathrm{SR}=0.86 \mathrm{EPS} \mathrm{C} / \mathrm{s}$

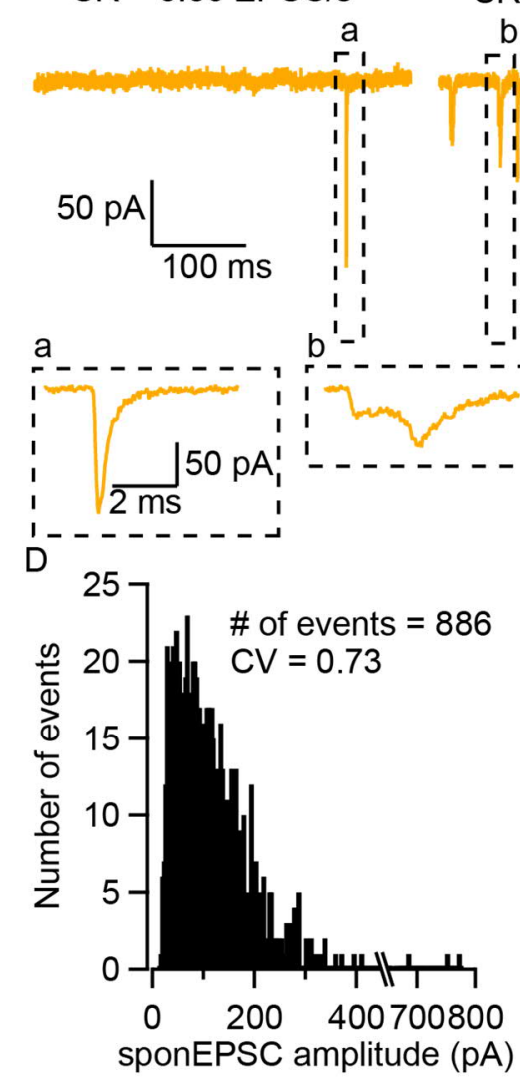

$\mathrm{H}$

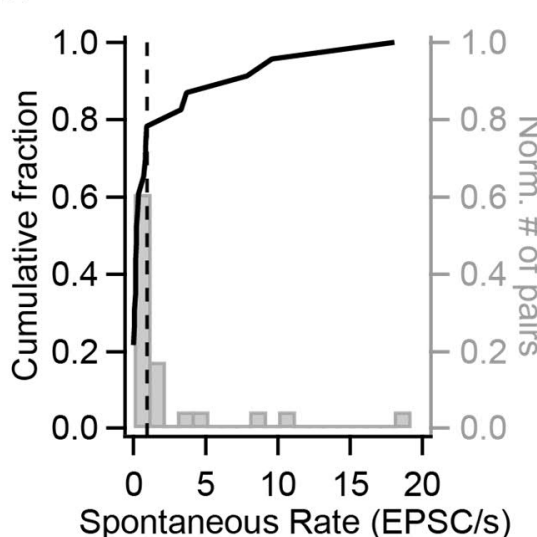

Spontaneous Rate (EPSC/s)

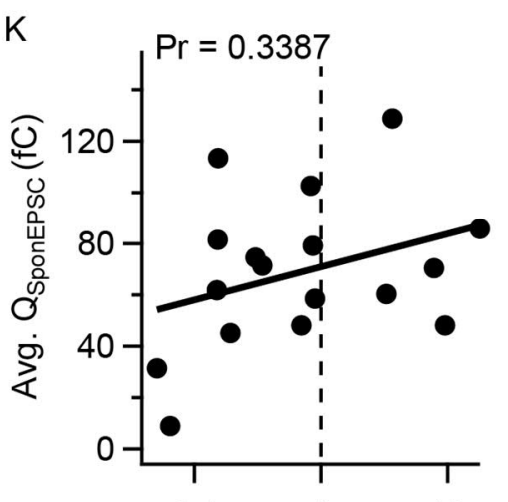

$\begin{array}{lll}0.1 & 1 & 10\end{array}$

Spontaneous Rate (EPSC/s)

$\mathrm{RR}=3.66 \mathrm{EPSC} / \mathrm{s}$

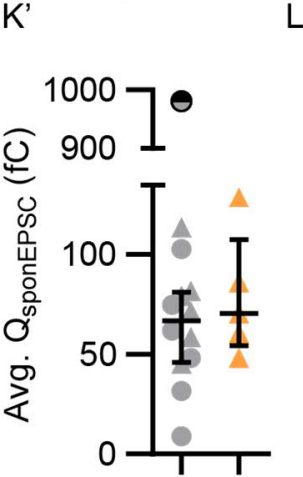

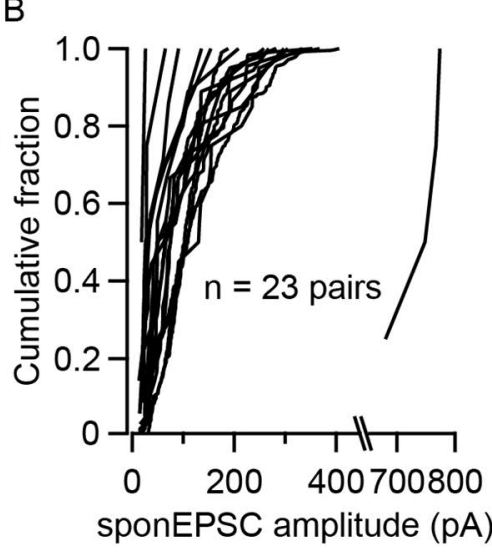

C
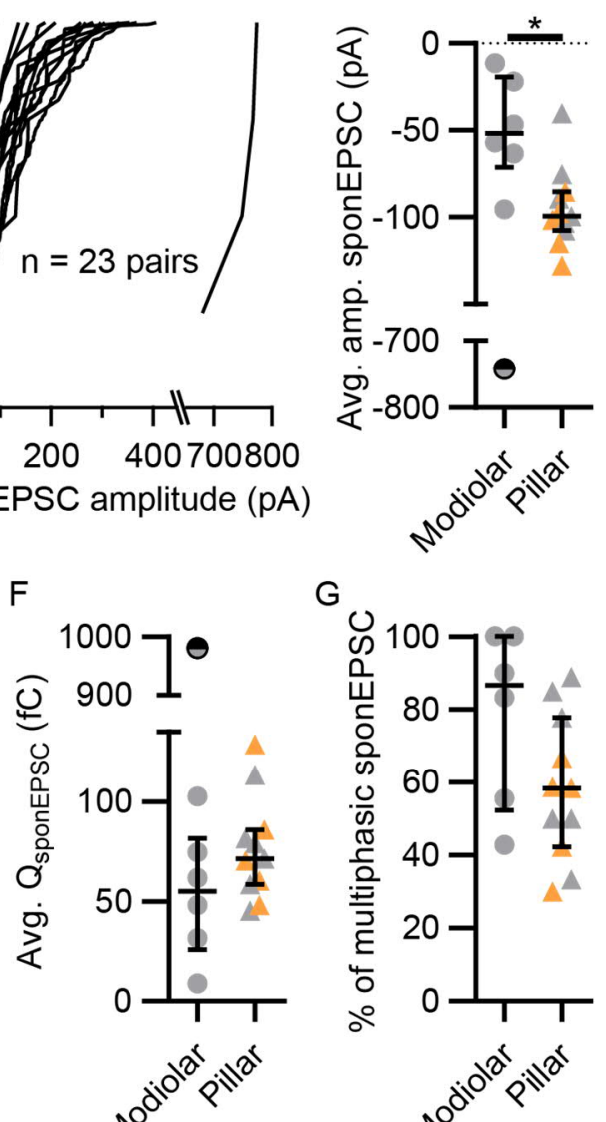

G
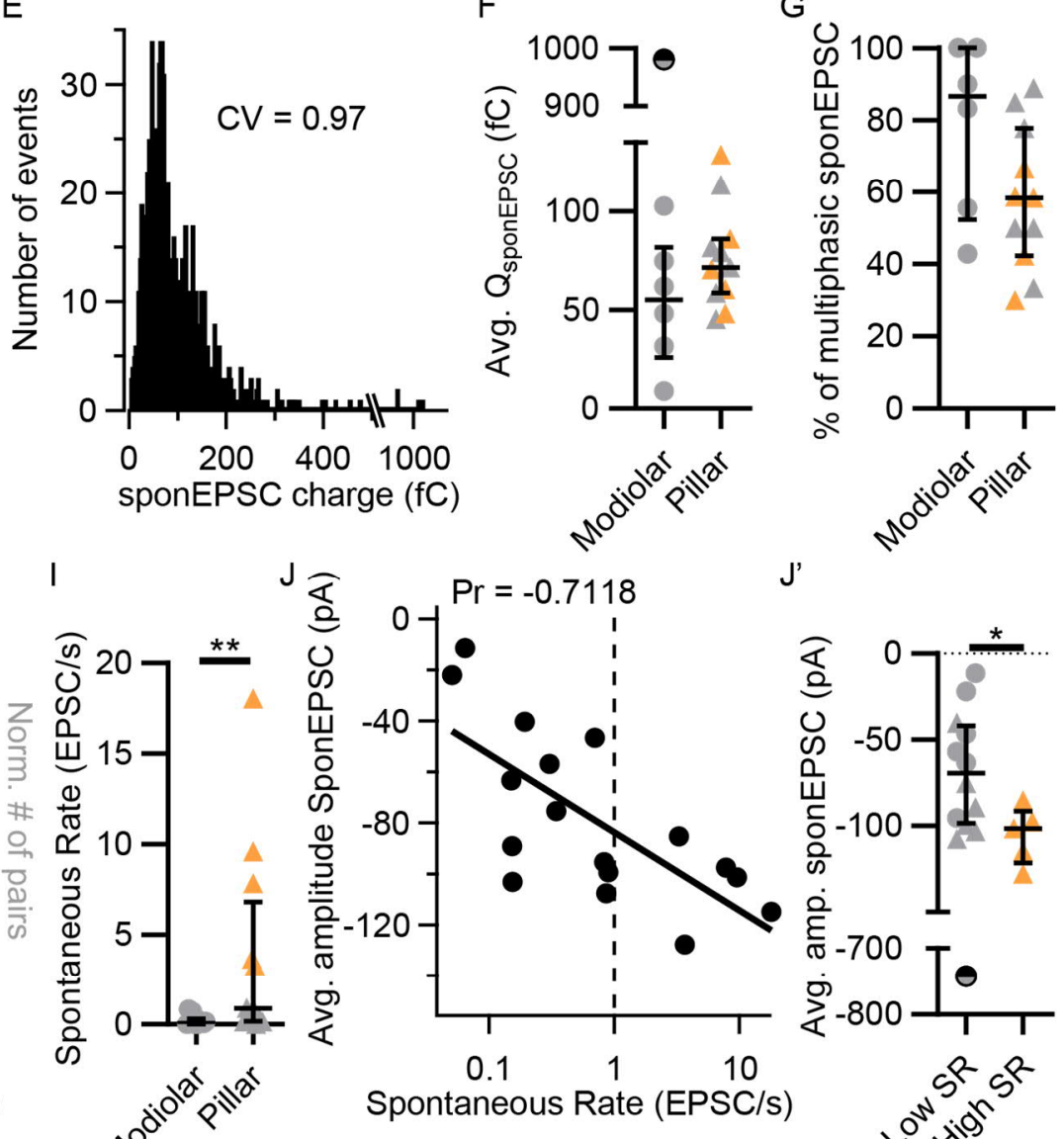

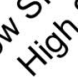
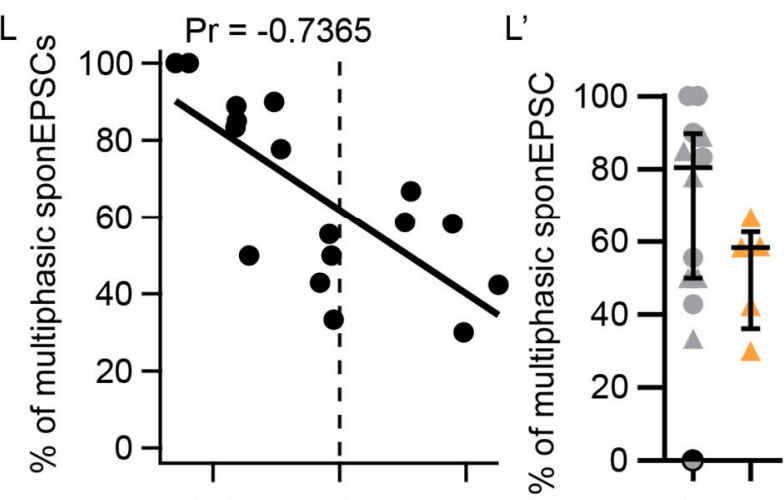

Spontaneous Rate (EPSC/s) $s^{2} s^{p}$

20 


\section{|Figure 4| Pillar synapses have higher rates of spontaneous release with larger SponEPSCs}

A. Spontaneous EPSCs recorded in the absence of stimulation (i.e. $\mathrm{IHC}$ holding potential $=-58 \mathrm{mV}$ ) from two exemplary paired recordings with different spontaneous rate. 'Pair \#' indentifies individual paired recordings. Insets show the selected sponEPSCs in an expanded time scale. (a,c) correspond to typical monophasic EPSCs (b) represents a multiphasic EPSC. B. Cumulative sponEPSC amplitude plots for 23 paired recordings. C. Synapses contacting the pillar face of the IHC had higher average amplitudes of sponEPSCs $(p=0.0104$, Mann-Whitney test). D-E. Pooled sponEPSC amplitude and charge distributions. F-G. Average sponEPSC charge (F) and percentage of multiphasic sponEPSCs $(\mathbf{G})$ in individual synapses recorded from the pillar or modiolar side of the IHC. H. Cumulative fraction (left axis) and normalized histogram (right axis) of the spontaneous rate (bin size is $1 \mathrm{EPSC} / \mathrm{s}$ ). I. Pillar synapses had higher rates of sponEPSCs ( $p=0.0086$, MannWhitney test). J,J'. Synapses with the highest SRs also had the largest sponEPSCs. (J) Spearman's correlation coefficient $=-0.7118$. (J') $p=0.0398$, Mann-Whitney test. K,K'. Average sponEPSC charge was not correlated to SR. L, L'. The percentage of multiphasic EPSCs was inversely correlated to SR (Spearman's correlation coefficient $=-0.7365$ ).

Scatter plots represent the $25^{\text {th }}, 50^{\text {th }}$ and $75^{\text {th }}$ percentiles with the individual data points overlaid. Synapses were classified as $\triangle$ pillar or O modiolar, and as Low SR $<1 \mathrm{EPSC} / \mathrm{s}$ or High SR $\geq 1 \mathrm{EPSC} / \mathrm{s}$.

Next, we calculated the rate of sponEPSC (SR) for each paired recording during 400 or 600 $\mathrm{ms}$ before the step depolarization protocols, or during 5 or $10 \mathrm{~s}$ of a continuous recording, in each case at a presynaptic holding potential of $-58 \mathrm{mV}$. SRs ranged from 0 to $18 \mathrm{EPSC} / \mathrm{s}$ |Figure $4 \mathrm{H} \mid$. The distribution of the SR was highly skewed: $75 \%$ of the pairs had rates lower than $1 \mathrm{EPSC} / \mathrm{s}$ and the median was $0.2 \mathrm{EPSC} / \mathrm{s}$. SR was significantly higher in boutons recorded from the pillar side (mean SR of $3.75 \pm 1.60 \mathrm{EPSC} / \mathrm{s}$ ) compared to the modiolar side (mean SR of $0.21 \pm 0.09 \mathrm{EPSC} / \mathrm{s} ; p=0.0086$, Mann-Whitney test) $\mid$ Figure $4 \mathrm{I} \mid$. Following Taberner and Liberman (2005), recordings were further classified as synapses with low $(<1$ sponEPSC/s) or high ( $\geq 1$ sponEPSC/s) SR. Synapses with the highest SRs also had the largest sponEPSCs (Spearman's correlation coefficient $=-0.7118$; average EPSC amplitude of $-67.57 \pm 9.43$ pA for low SR vs $-105.4 \pm 7.35$ pA for high SR synapses; $p=0.0398$, MannWhitney test) |Figure 4J, J'|. Contrary, the average charge of the sponEPSCs was comparable regardless the SR (Spearman's correlation coefficient $=0.3387$; charge of $64.74 \pm 8.43 \mathrm{fC}$ for low SR vs $78.72 \pm 13.97 \mathrm{fC}$ for high SR synapses; $p=0.5621$, Mann-Whitney test) |Figure 4K, K'|. The percentage of multiphasic EPSCs was inversely correlated to the rate of spontaneous release (Spearman's correlation coefficient $=-0.7365$ ) $\mid$ Figure $4 \mathrm{~L} \mid$, even though there was no significant difference when compared between low and high SR synapses |Figure 4L'|. Taken together, these results suggest that pillar synapses have higher rates of spontaneous release with larger and more compact sponEPSCs. 


\section{Synaptic vesicle pool dynamics}

In 8 (all from the pillar side) of the 23 aforementioned pairs, we studied IHC synaptic vesicle pool dynamics. We used a modified version of the forward masking paradigm that has been introduced for in vivo analysis of SGN spike rate adaptation and recovery from adaptation (Harris and Dallos, 1979). Applied to recordings of EPSCs, the protocol provides direct experimental assessment of depression and recovery of the readily releasable pool of vesicles (RRP). Given its potential presynaptic effects (Diamond and Jahr, 1995; Dittman and Regehr, 1998), we did not employ cyclothiazide to inhibit AMPA receptor desensitization, which also contributes to synaptic depression (Goutman, 2017). The paradigm consisted of a set of two strong step IHC depolarizations (to $-17 \mathrm{mV}$ ) separated by different interstimulus intervals (ISI: 4, 16, 64 and $256 \mathrm{~ms}$ ) |Figure 5A|. The first stimulus - called masker, as it depresses the response to a subsequent stimulus when applied in rapid succession - had a duration of $100 \mathrm{~ms}$. The second stimulus (denominated probe) lasted for $15 \mathrm{~ms}$. The recordings included a time frame of $400 \mathrm{~ms}$ preceeding the masker and $400 \mathrm{~ms}$ following the probe. The interval between masker and masker was $20 \mathrm{~s}$, and each protocol was repeated between 3 to 20 times.

The synaptic response to the masker stimulus had a mean latency of $1.27 \pm 0.09 \mathrm{~ms}$, calculated from the onset of the voltage step to the onset of the triggered EPSC. The EPSC charge (QEPSC) in response to the first $50 \mathrm{~ms}$ of the masker was fitted by the sum of a single exponential and a line function (discontinuous lines in $\mid$ Figure $5 \mathrm{~B} \mid$ ). The amplitude of the exponential, thought to reflect RRP exocytosis, was on average $0.97 \pm 0.18 \mathrm{pC}$. The linear component had on average a slope of $32.31 \pm 5.55 \mathrm{pC} / \mathrm{s}$, reporting the rate of sustained exocytosis.

To quantify synaptic release in terms of vesicles, we used the average charge of sponEPSCs recorded for each pair. This assumes that each sponEPSC corresponds to a unitary release event ("univesicular mode of release" (Chapochnikov et al., 2014)). On average, $12.64 \pm$ 1.68 vesicles constituted the RRP, which was depleted with a mean time constant of $6.47 \pm$ $1.03 \mathrm{~ms}$. The mean initial rate of release was $2400 \pm 600$ vesicles/s, while the mean rate of sustained exocytosis was $445.7 \pm 87.92$ vesicles/s. Neither the dynamics of synaptic vesicle release, nor the integral of the IHC Ca ${ }^{2+}$ influx $\left(\mathrm{Q}_{\mathrm{Ca}}\right)$, significantly differed between low and high SR synapses in our relatively small data set (|Figure $5 \mathrm{C}-\mathrm{H}, \mathrm{J} \mid$; table 1). High SR synapses, however, were slightly faster, with higher initial release rates and had a tendency towards a stronger adaptation of the release rate after the initial peak (|Figure $5 \mathrm{H} \mid$; table 1). 
A
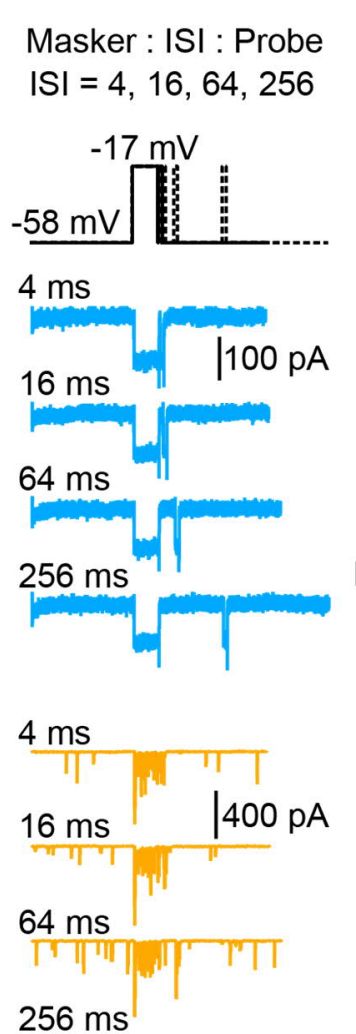

$256 \mathrm{~ms}$
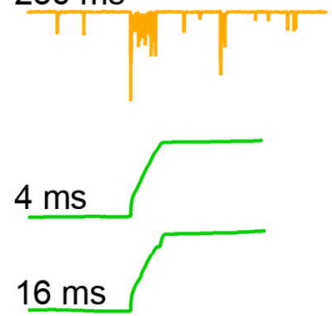

$64 \mathrm{~ms}$

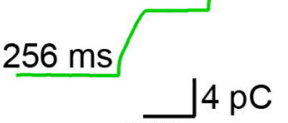
$200 \mathrm{~ms}$

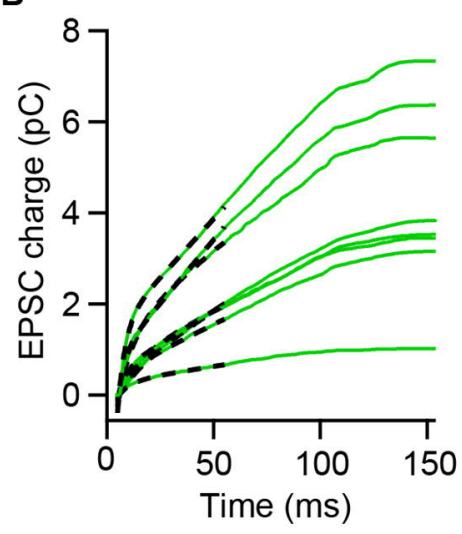

$\mathbf{F}$

$$
\text { J }
$$

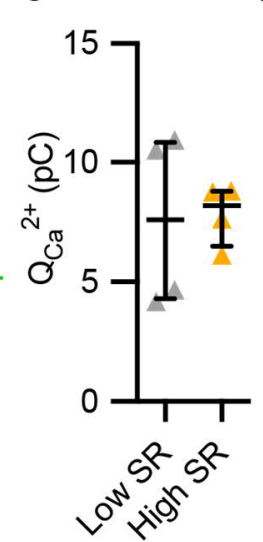

$\mathrm{K}$

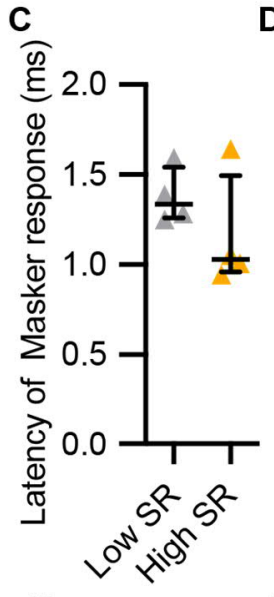

D

E

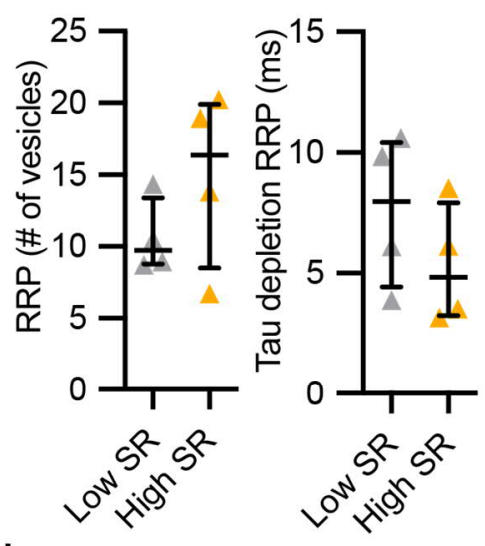

H

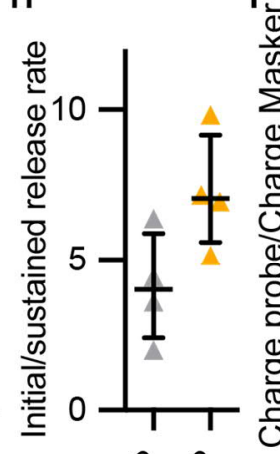

क्र $s^{2}$

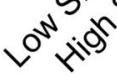
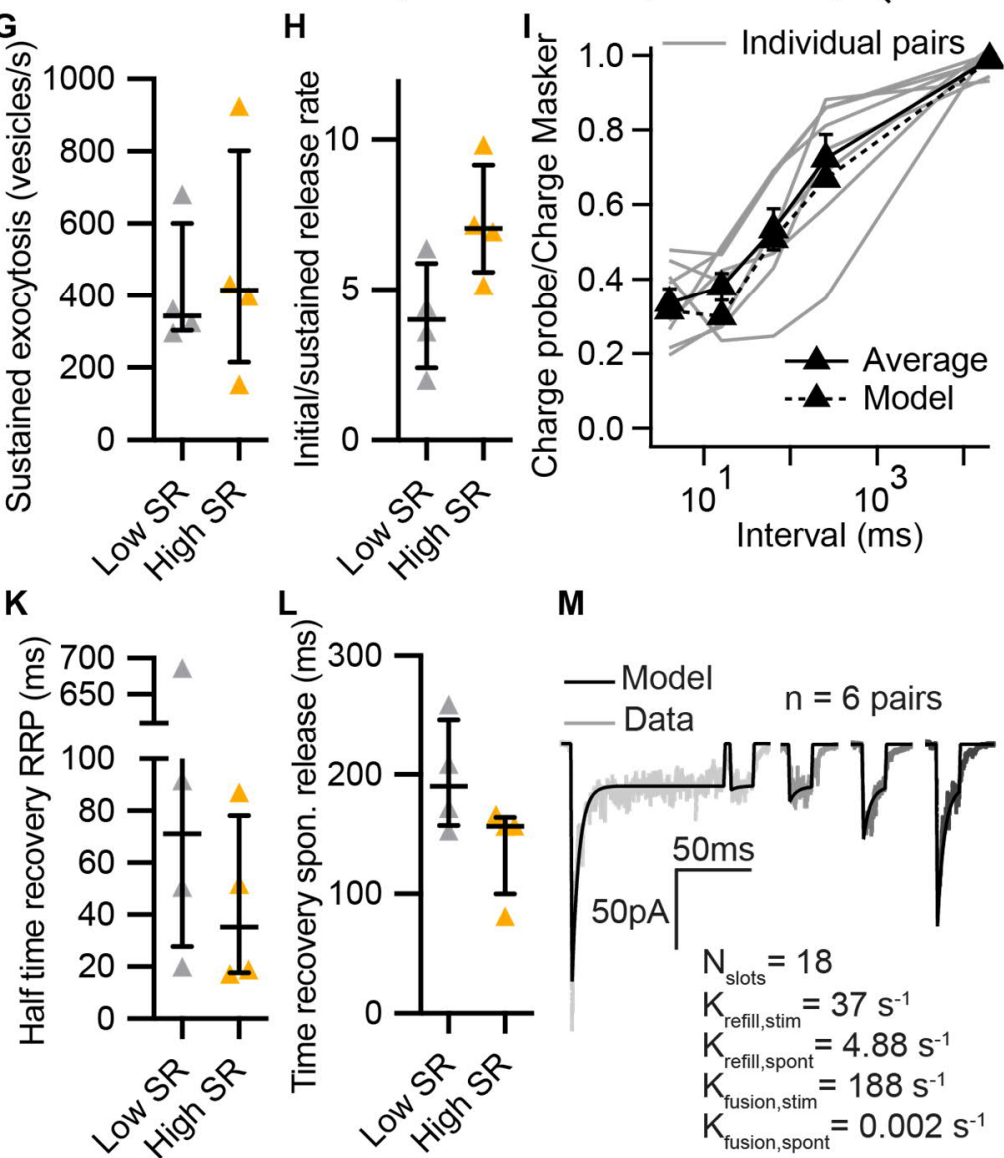

M

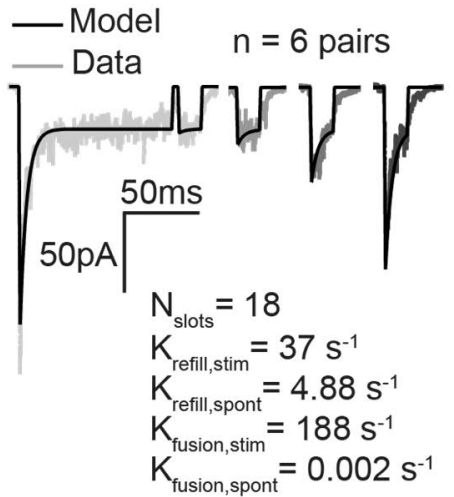

|Figure 5| Synaptic vesicle pool dynamics of individual IHC synapses

A. Representative responses of $\mathrm{Ca}^{2+}$ influx (blue), EPSC (light orange) and QEPSC (green) to forward masking protocols used to study depression and recovery of RRP. The stimulus (top panel) consists of two sequential voltage steps (masker and probe) separated by different interstimulus intervals (ISI in ms). B. Pool depletion dynamics were studied by fitting the sum of a single exponential and a line function (black discontinuous line) to the first $50 \mathrm{~ms}$ of average QEPSC in response to the masker. C. Synaptic delay as the latency between stimulus onset and EPSC onset. D-H. RRP, tau of depletion, initial release rate and sustained release were calculated from the fits and the average $\mathrm{Q}_{\text {spon EPSC }}$ for each pair. I. Recovery from RRP depletion shown as ratio of QEPSC probe and QEPSC masker (mean \pm sem) during the first $10 \mathrm{~ms}$ of the stimulus. J. $\mathrm{Ca}^{2+}$ charge during the masker. K. Half time of recovery from RRP depletion calculated from the plots in (I). L. Time to recovery of the spontaneous release after the probe offset. M. Averaged trace of the aligned responses of 6 pairs was fitted with an adapted version of a previously developed model of RRP pool dynamics (black line) (Jean et al., 2018). The refilling ( $\left.\mathrm{K}_{\text {refill }}\right)$ and fusion $\left(\mathrm{K}_{\text {fusion }}\right)$ rate constant during spontaneous (spont) and stimulated (stim) conditions as well as the number of occupied release sites ( $\left.\mathrm{N}_{\text {slots }}\right)$ are provided. 
Scatter plots represent the $25^{\text {th }}, 50^{\text {th }}$ and $75^{\text {th }}$ percentiles with the individual data points overlaid. Synapses were classified as $\triangle$ pillar or O modiolar, and as Low SR $<1 \mathrm{EPSC} / \mathrm{s}$ or High $\mathrm{SR} \geq 1 \mathrm{EPSC} / \mathrm{s}$.

Next, we determined the QEPSC amplitude in the first $10 \mathrm{~ms}$ of both masker and probe stimuli.

The ratio of probe and masker responses was plotted against each interstimulus interval (ISI), including the masker-to-masker interval $(20 \mathrm{~s}$, |Figure $5 \mathrm{I} \mid)$. We determined the half time of the recovery from RRP depletion. Synapses with low SR tended to recover slower than high SR ones (half time of recovery was $212 \pm 158 \mathrm{~ms}$ vs. $43 \pm 16 \mathrm{~ms}$, respectively; |Figure 5K|; table 1). Spontaneous activity was also resumed faster in high SR synapses $(198.1 \pm 23.48$ ms for low SR vs $140.3 \pm 19.80 \mathrm{~ms}$ for high SR synapses; |Figure 5L|; table 1) even though this difference is not statistically significant in our dataset.

Table 1. Parameters of release dynamics for low and high SR synapses obtained from forward masker paradigms

\begin{tabular}{lccc}
\hline \multicolumn{1}{c}{ Parameter } & Low SR pair & High SR pair & p-value \\
\hline \hline Latency of Masker response (ms) & $1.38 \pm 0.08$ & $1.16 \pm 0.16$ & 0.3429 \\
\hline Amp exponential component (pC) & $0.71 \pm 0.05$ & $1.24 \pm 0.33$ & 0.3429 \\
\hline Slope linear component (pC/s) & $26.70 \pm 1.8$ & $37.92 \pm 10.93$ & 0.3429 \\
\hline RRP (vesicles) & $10.63 \pm 1.30$ & $14.93 \pm 3.06$ & 0.4857 \\
Tau of depletion of RRP (ms) & $7.61 \pm 1.59$ & $5.33 \pm 1.25$ & 0.3429 \\
\hline Initial release rate (vesicles/s) & $1540 \pm 269$ & $3411 \pm 1155$ & 0.3429 \\
\hline Sustained exocytosis (vesicles/s) & $416 \pm 89$ & $477 \pm 161$ & 0.6857 \\
\hline Initial/sustained release rate & $4.09 \pm 0.91$ & $7.26 \pm 0.97$ & 0.0571 \\
\hline QCa (pC) & $7.58 \pm 1.82$ & $7.82 \pm 0.63$ & $>0.999$ \\
\hline Half time of RRP recovery (ms) & $212.2 \pm 158.8$ & $43.71 \pm 16.46$ & 0.3429 \\
\hline Recovery time of spon. release (ms) & $198.1 \pm 23.48$ & $140.3 \pm 19.80$ & 0.2000
\end{tabular}

Finally, we adapted a previously developed biophysical model of RRP pool dynamics (Frank et al., 2010; Jean et al., 2018; Jung et al., 2015) to extract information regarding the number of vesicular release sites of the RRP ( $\left.\mathrm{N}_{\text {slots }}\right)$, as well as stimulus-dependent rates (fusion and replenishment rate constants per release site in the presence and absence of stimulation). The model was fitted to the average trace of the aligned responses of 6 pairs for each ISI (|Figure $5 \mathrm{M} \mid$; for this analysis, we selected pairs for which the forward masking paradigm was repeated at least five times). The results of the model fitting suggested 18 release sites per 
synapse, each release site with fusion and refill rate constants during stimulation of $188 \mathrm{~s}^{-1}$ and of $37 \mathrm{~s}^{-1}$, respectively.

\section{Stimulus intensity coding at IHC synapses}

IHC synapses encode stimulus intensity. Average discharge rates of auditory nerve fibers recorded in vivo increase sigmoidally with the strength of the acoustic stimuli (Sachs and Abbas, 1974; Taberner and Liberman, 2005; Winter et al., 1990). To further understand stimulus intensity coding in mouse IHC synapses, we measured $\mathrm{Ca}^{2+}$ currents and EPSCs in response to $10 \mathrm{~ms}$ depolarizations to different potentials ranging from -58 to $-18 \mathrm{mV}$ in 5 $\mathrm{mV}$ steps |Figure 6A|. We used step depolarizations instead of a sinewave depolarization given that mature and high frequency IHCs have graded receptor potentials that represent the rectified envelope of an acoustic stimulus (i.e. the DC component) (Russell and Sellick, 1978). The voltage dependence of $\mathrm{Ca}^{2+}$ channel activation was studied by fitting a Boltzmann function to the activation curves |Figure 6B, C|. Similarly, the voltage dependence of synaptic vesicle release per active zone was approximated by fitting a sigmoidal function to the individual rate-level plots |Figure 6B, G|. One postsynaptic bouton was recorded per IHC. Two out of 20 paired recordings were excluded from this analysis due to failure to fit a sigmoidal function (assessed by visual inspection).

Pairs with low SR $(<1 \mathrm{EPSC} / \mathrm{s})$ exhibited a higher voltage threshold of release $(10 \%$ of the maximum release $-\mathrm{Q}_{10}$, EPSC- occurred at $-48.71 \pm 0.88 \mathrm{mV}$ ) compared to boutons with high $\mathrm{SR}\left(\mathrm{Q}_{10}\right.$ at $-57.12 \pm 0.98 \mathrm{mV} ; p=0.0017$, Mann-Whitney test) $\mid$ Figure $6 \mathrm{H} \mid$. The voltage of half-maximal release ( $\mathrm{V}_{\text {half }} \mathrm{Q}_{\mathrm{EPSC}}$ ) of low SR boutons $(-42.30 \pm 1.12 \mathrm{mV})$ also displayed a depolarized shift of $\sim 9 \mathrm{mV}$ compared to high SR boutons $(-51.37 \pm 1.15 \mathrm{mV} ; p=0.0017$, Mann-Whitney test) |Figure 6I|. The voltage sensitivity of vesicle release, determined by a slope factor, was similar between high and low SR synapses $\mid$ Figure $6 \mathrm{~J} \mid$. Interestingly, IHCs from which low SR boutons were recorded also displayed a small but significant depolarized shift in the voltage dependence of whole-cell $\mathrm{Ca}^{2+}$ current activation ( $\mathrm{V}_{\text {half }} \mathrm{I}_{\mathrm{Ca}}$ of $-37.73 \pm$ $0.83 \mathrm{mV}$ vs $-41.55 \pm 1.04 \mathrm{mV}$ in IHCs from which high SR boutons were recorded; $p=$ 0.0184 , Mann Whitney test) |Figure 6E|. The voltage sensitivity of the whole-cell $\mathrm{Ca}^{2+}$ influx, as well as the voltage-threshold for $\mathrm{Ca}^{2+}$ influx, were similar regardless the SR of the accompanying bouton |Figure 6D, F|. Dynamic ranges, defined as the voltage range for which the exocytosis changes from 10-90\%, were comparable regardless of the SR of the synapses (15.28 $\pm 1.512 \mathrm{mV}$ for low SR boutons vs $14.45 \pm 2.99 \mathrm{mV}$ for high SR boutons) |Figure 6K|. 
A Pair \#140817_1

Pair \#1200618_1

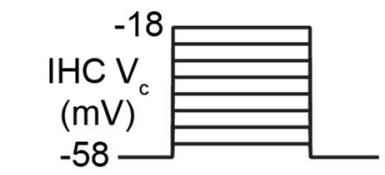

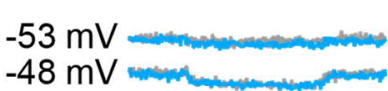

$-43 \mathrm{mV}$

$-38 \mathrm{mV}$

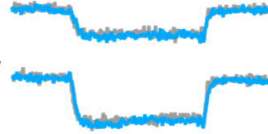

$-33 \mathrm{mV}=\mathrm{m}$

$-28 \mathrm{mV}$.

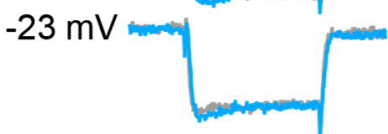

$-18 m V=1$
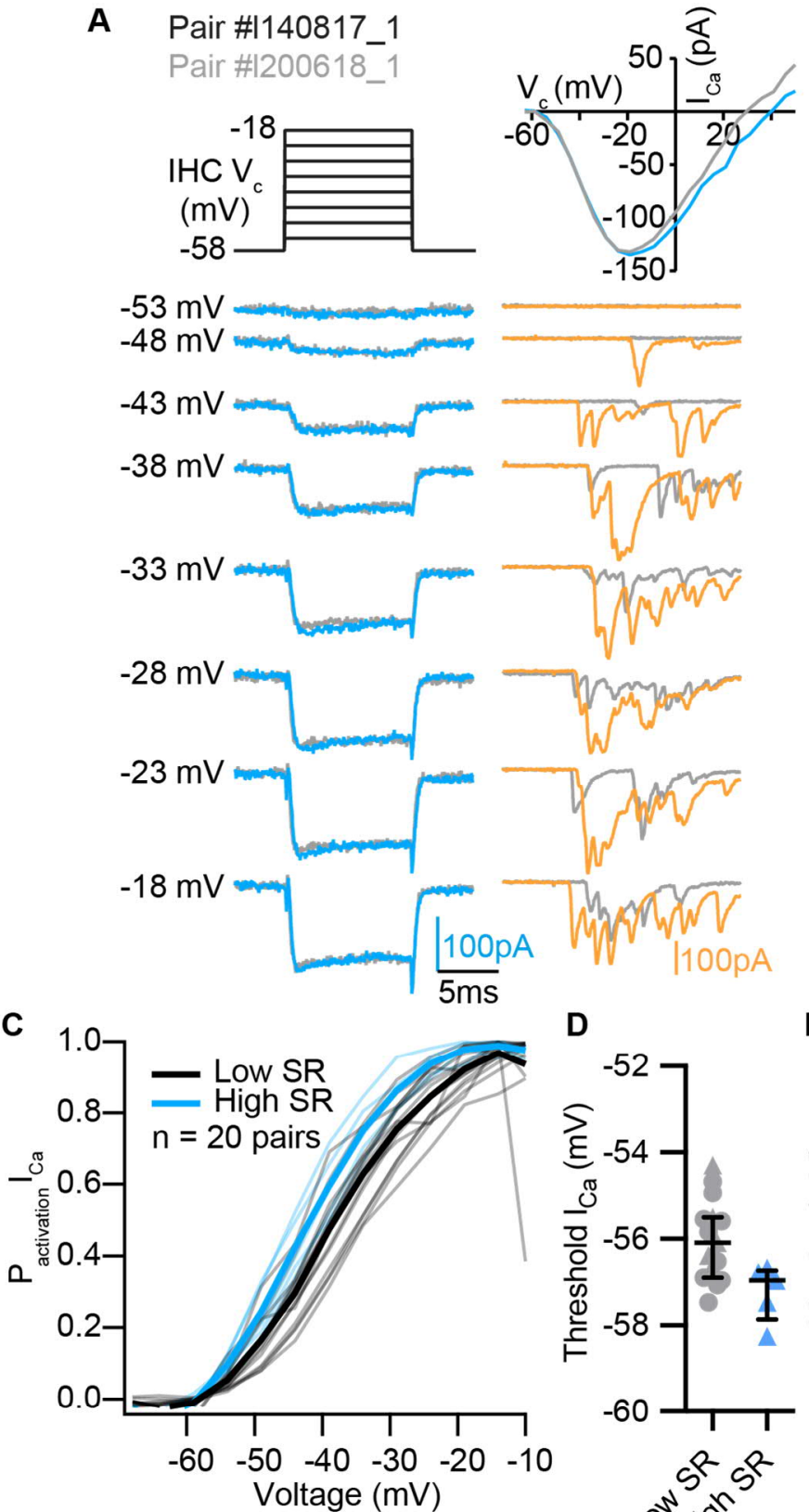

B
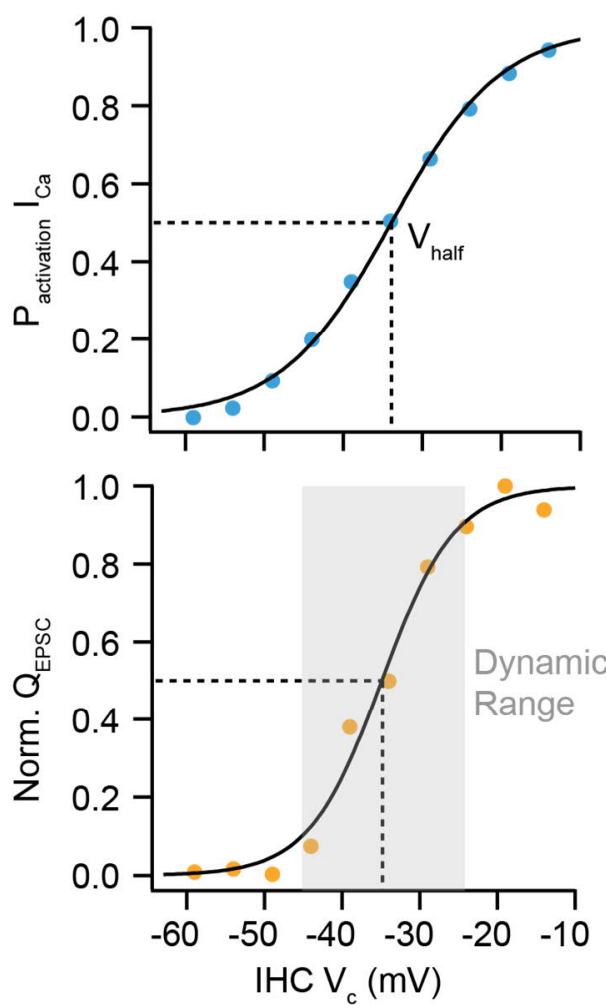

E

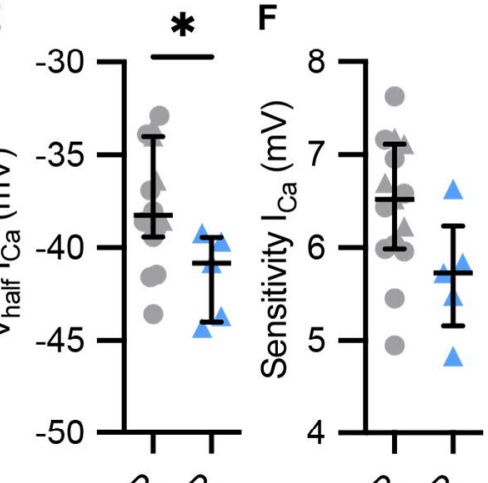

$s^{2} s^{2}$

20 in

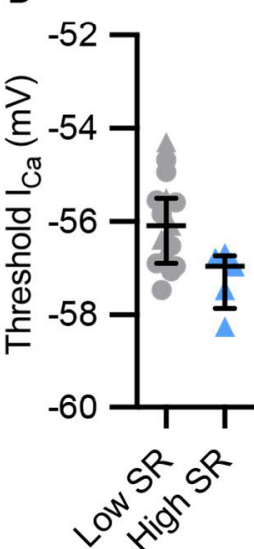

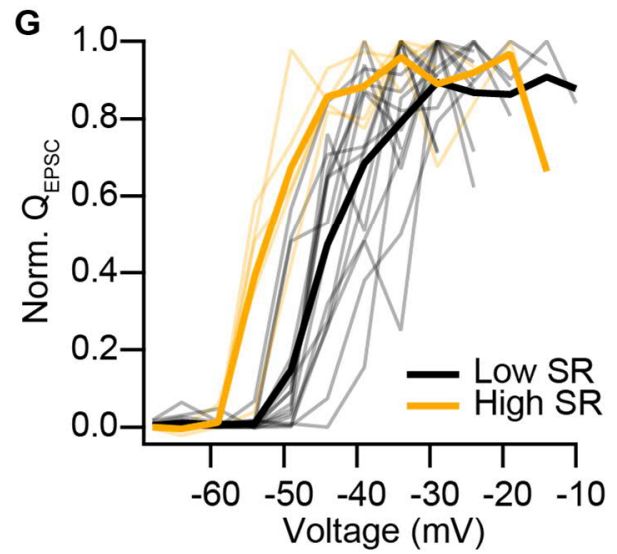

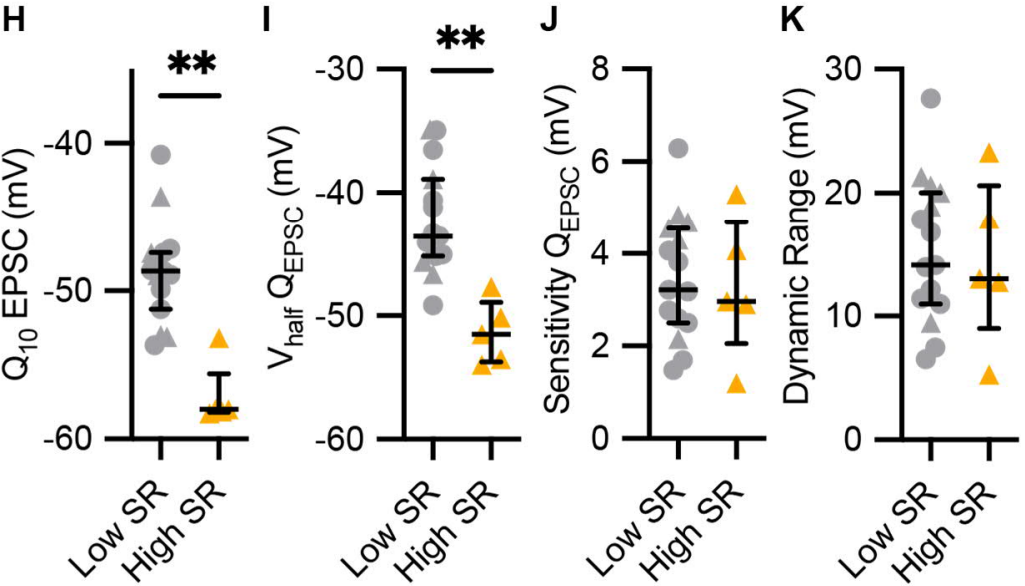

|Figure 6| Voltage dependence of IHC $\mathrm{Ca}^{2+}$ influx and synaptic release

A. IHC $\mathrm{Ca}^{2+}$ current (blue traces) and EPSCs (orange traces) of two examplary pairs in response to $10 \mathrm{~ms}$ depolarizations to different potentials ranging from -58 to $-18 \mathrm{mV}$ in $5 \mathrm{mV}$ steps (upper left panel). The upper 
right panel shows the current-voltage relationships for the two examplary pairs. B. Upper panel: Fractional activation of the $\mathrm{Ca}^{2+}$ channels ( $\mathrm{P}_{\text {activation}}$, blue data points) was obtained from the normalized chord conductance. Voltage of half-maximal activation $\left(\mathrm{V}_{\text {half }} \mathrm{Ca}\right.$ ) and voltage sensitivity of the $\mathrm{Ca}^{2+}$ current (slope) were determined using a Boltzmann fit (black trace) to the activation curve. Lower panel: Rate level curves (orange data points) were obtained from the QEPSC for each depolarization step. A sigmoidal function (black trace) was fitted to obtain the voltage of half-maximal synaptic release ( $\left.\mathrm{V}_{\text {half }} \mathrm{QEPSC}_{\mathrm{EP}}\right)$ and the voltage sensitivity of the release (slope), as well as the dynamic range for which the exocytosis changes from 10-90\% (gray area). C, D. Voltage dependence of whole-cell $\mathrm{Ca}^{2+}$ channel activation (activation curve; $\mathbf{C}$ ) and triggered single active zone EPSCs (rate level curve; G) for 23 pairs of low ( $\leq 1 \mathrm{EPSC} / \mathrm{s})$ and high SR $(>1 \mathrm{EPSC} / \mathrm{s})$. Averages (thick lines) and individual curves (thin lines) are overlaid. D-F. The threshold (D), $\mathrm{V}_{\text {half }} \mathrm{I}_{\mathrm{Ca}}$ (E) and voltage sensitivity of the $\mathrm{Ca}^{2+}$ current (F) from the activation curves in C. H-J. Voltage of $10 \%$ of maximum release

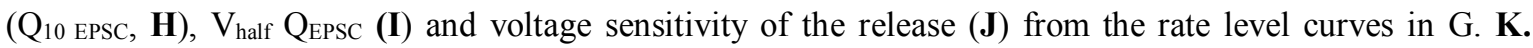
Dynamic range from pairs shown in $\mathrm{G}$.

Scatter plots represent the $25^{\text {th }}, 50^{\text {th }}$ and $75^{\text {th }}$ percentiles with the individual data points overlaid. Synapses were classified as $\triangle$ pillar or O modiolar, and as Low SR $<1$ EPSC/s or High SR (blue when it referes to $\mathrm{Ca}^{2+}$ influx parameters) $\geq 1 \mathrm{EPSC} / \mathrm{s}$.

\section{Apparent $\mathrm{Ca}^{2+}$ dependence of neurotransmitter release}

The coupling between $\mathrm{Ca}^{2+}$ influx and exocytosis critically determines how acoustic stimuli are encoded at the synapse between the IHC and the SGN (Moser and Vogl, 2016). Therefore, we were interested in understanding the $\mathrm{Ca}^{2+}$ dependence of IHC neurotransmitter release. The $\mathrm{Ca}^{2+}$ nanodomain hypothesis of exocytosis control proposes that one $\mathrm{Ca}^{2+}$ channel in nanometer proximity from the vesicular release site governs the $\mathrm{Ca}^{2+}$ concentration that drives the release of a synaptic vesicle [reviewed in (Eggermann et al., 2012; Moser et al., 2006b)]. As data and modeling indicated that the $\mathrm{Ca}^{2+}$ at the $\mathrm{Ca}^{2+}$ sensor of exocytosis is dominated by one channel, but few other channels still contribute in IHC exocytosis (partial domain overlap), the control has been coined " $\mathrm{Ca}^{2+}$ nanodomainlike" hypothesis (Brandt et al., 2005; Pangršič et al., 2015; Wong et al., 2014). However, this was based on membrane capacitance measurements summing over all IHC synapses. A previous study using pre- and postsynaptic recordings from immature rat IHCs also supported a $\mathrm{Ca}^{2+}$ nanodomain-like control of exocytosis (Goutman and Glowatzki, 2007). To our knowledge, this hypothesis has not yet been tested by paired pre- and postsynaptic recordings in mice after the onset of hearing. Moreover, it remained unclear whether the $\mathrm{Ca}^{2+}$ nanodomain-like control operates in IHC synaptic transmission under physiological conditions. Finally, most previous studies had used stimulus durations that cause partial depletion of the RRP, thereby violating the requirement for estimating the $\mathrm{Ca}^{2+}$ cooperativity based on the initial rate of release. Here, we addressed these open questions by determining the apparent $\mathrm{Ca}^{2+}$ cooperativity of vesicle release with brief stimuli while manipulating the 
single $\mathrm{Ca}^{2+}$ channel domain or the number of open $\mathrm{Ca}^{2+}$ channels of IHCs maintained at near-physiological conditions.

A

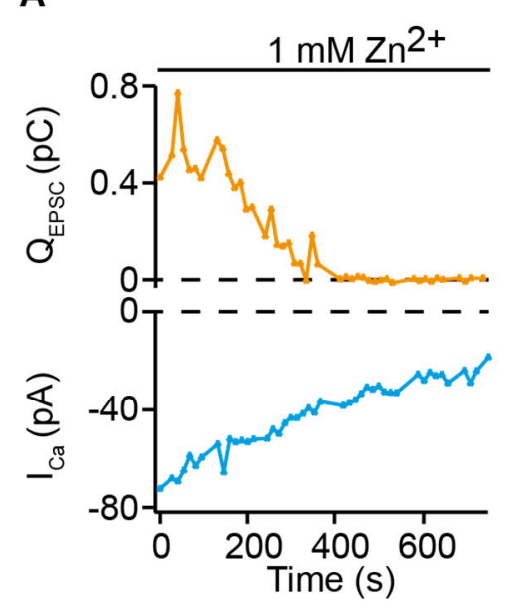

D
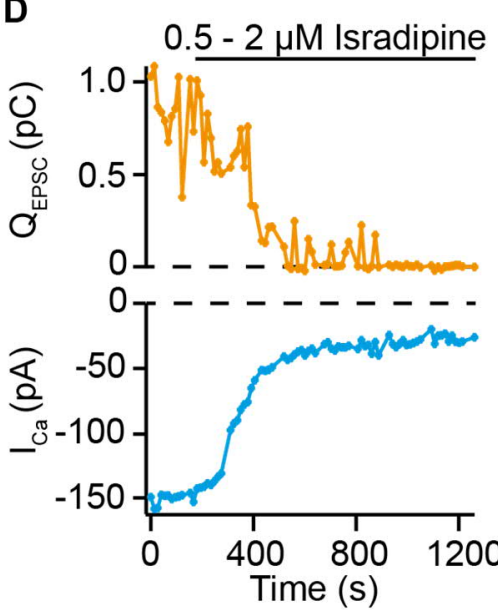

B

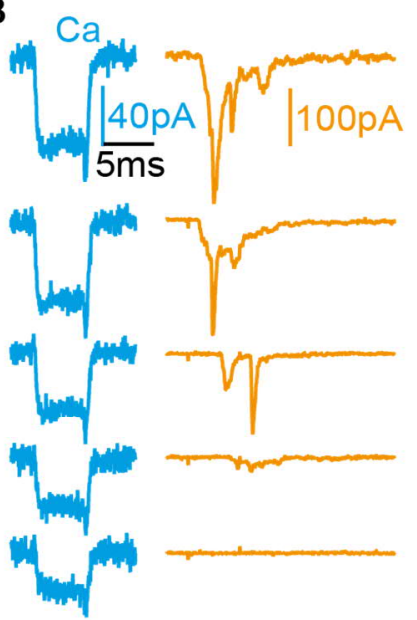

$E$

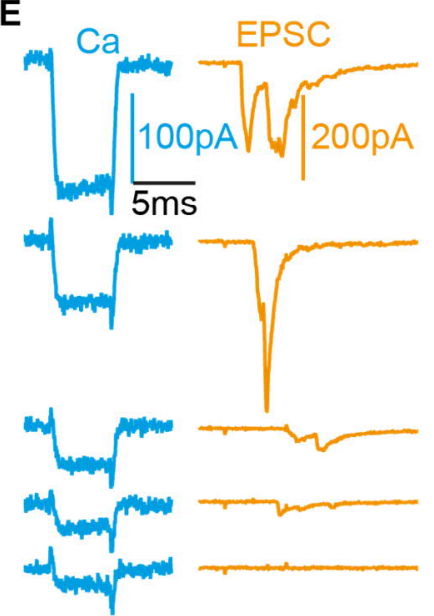

C

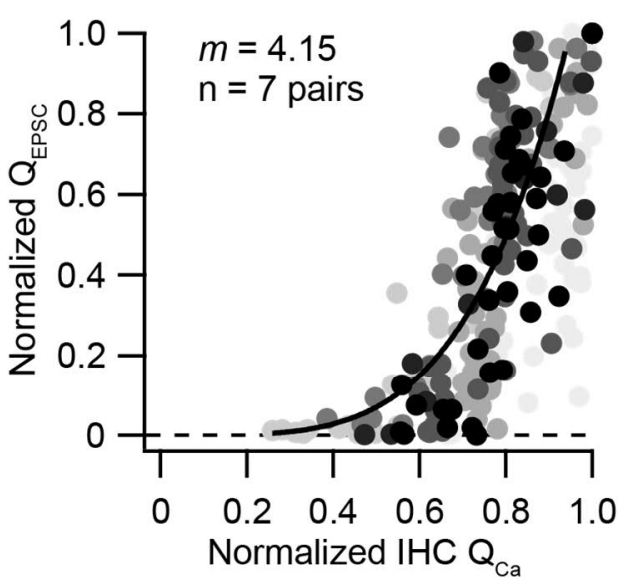

$\mathbf{F}$

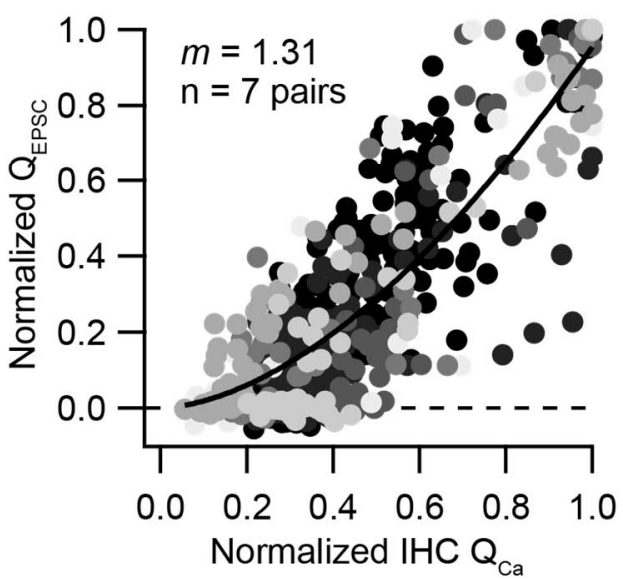

|Figure 7| Pharmacological approaches to manipulate the (apparent) single $\mathrm{Ca}^{2+}$ channel influx or the open $\mathrm{Ca}^{2+}$ channel number in IHCs

A, B. Slow perfusion of $1 \mathrm{mM} \mathrm{Zn}^{2+}$ reduced the effective single $\mathrm{Ca}^{2+}$ channel current and the concomitant neurotransmitter release evoked by $5 \mathrm{~ms}$ step depolarizations. C. Scatter plot of normalized $\mathrm{Ca}^{2+}$ current integrals $\left(\mathrm{Q}_{\mathrm{Ca}}\right)$ versus the corresponding normalized elicited EPSC charge ( $\left.\mathrm{Q}_{\mathrm{EPSC}}\right)$. The solid line is a leastsquares fit of a power of exponent function $\left(\mathrm{Q}_{\mathrm{EPSC}}=\mathrm{a}+\mathrm{b}\left(\mathrm{Q}_{\mathrm{Ca}}\right)^{m}\right)$ revealed a supralinear relationship of neurotransmitter release ( $m=4.17 ; \mathrm{n}=7$ pairs). D-F. The slow reduction in the number of open $\mathrm{Ca}^{2+}$ channels achieved by perfusion of $0.5-2 \mu \mathrm{M}$ Isradipine, yielded a linear relationship between $\mathrm{Q}_{\mathrm{EPSC}}$ and $\mathrm{Q}_{\mathrm{Ca}}(m=1.31$, $\mathrm{n}=7$ pairs) as can be observed in $\mathbf{F}$.

We first varied the single channel current by slowly perfusing $1 \mathrm{mM} \mathrm{Zn}^{2+}$ to cause a rapid (microsecond scale) flicker block of the $\mathrm{Ca}^{2+}$ channel current (Winegar and Lansman, 1990) |Figure 7A, B|. We argue that this causes an apparent reduction of the fusogenic $\mathrm{Ca}^{2+}$ signal: while the current through the unblocked channel is not changed, the limited kinetics of the $\mathrm{Ca}^{2+}$ binding to the $\mathrm{Ca}^{2+}$ sensor leads to a temporal averaging of the flickering single $\mathrm{Ca}^{2+}$ channel current and thus the fusogenic $\mathrm{Ca}^{2+}$ domain around the channel. The relationship of 
QEPSC and $\mathrm{Ca}^{2+}$ charge (IHC $\mathrm{Q}_{\mathrm{Ca}}$ ) evoked by $5 \mathrm{~ms}$ long depolarizations was approximated by a power of exponent function $\left(\mathrm{Q}_{\mathrm{EPSC}}=\mathrm{a}+\mathrm{b}\left(\mathrm{Q}_{\mathrm{Ca}}\right)^{m}\right)$. This manipulation revealed a supralinear relationship between QEPSC and IHC $\mathrm{Q}_{\mathrm{Ca}}(m=4.15)$ |Figure $7 \mathrm{C} \mid$, most likely reflecting the high intrinsic $\mathrm{Ca}^{2+}$ cooperativity at the $\mathrm{Ca}^{2+}$ sensor (Beutner et al., 2001).
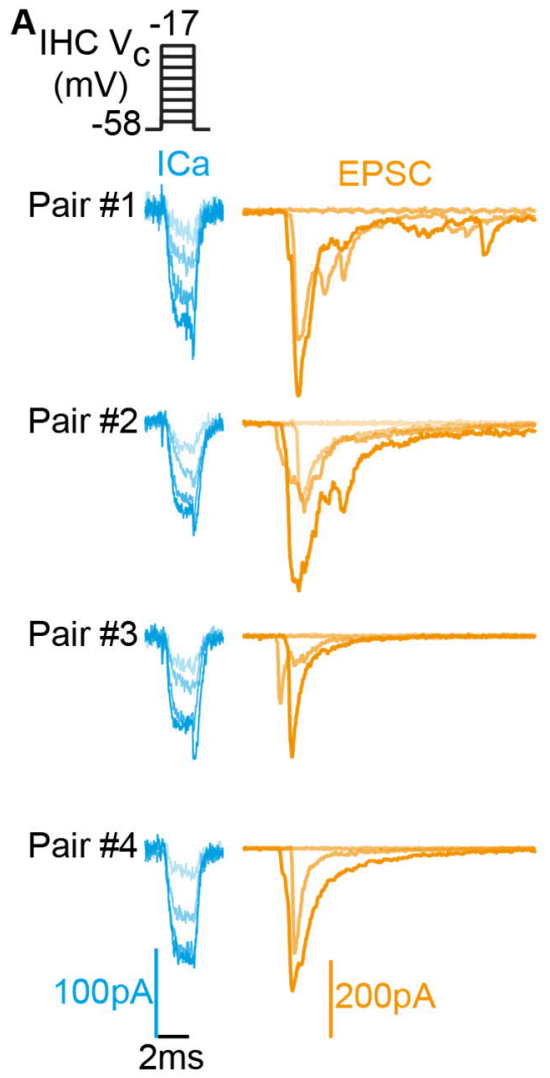
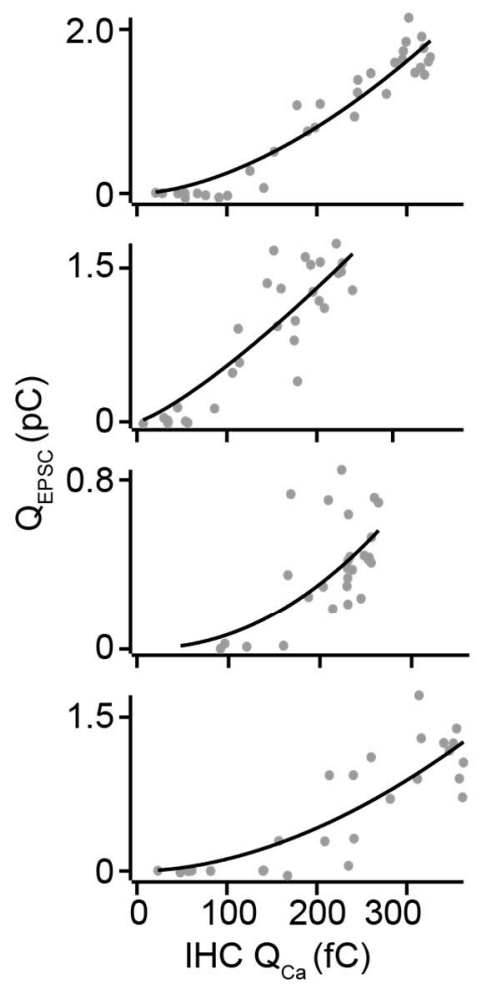

B
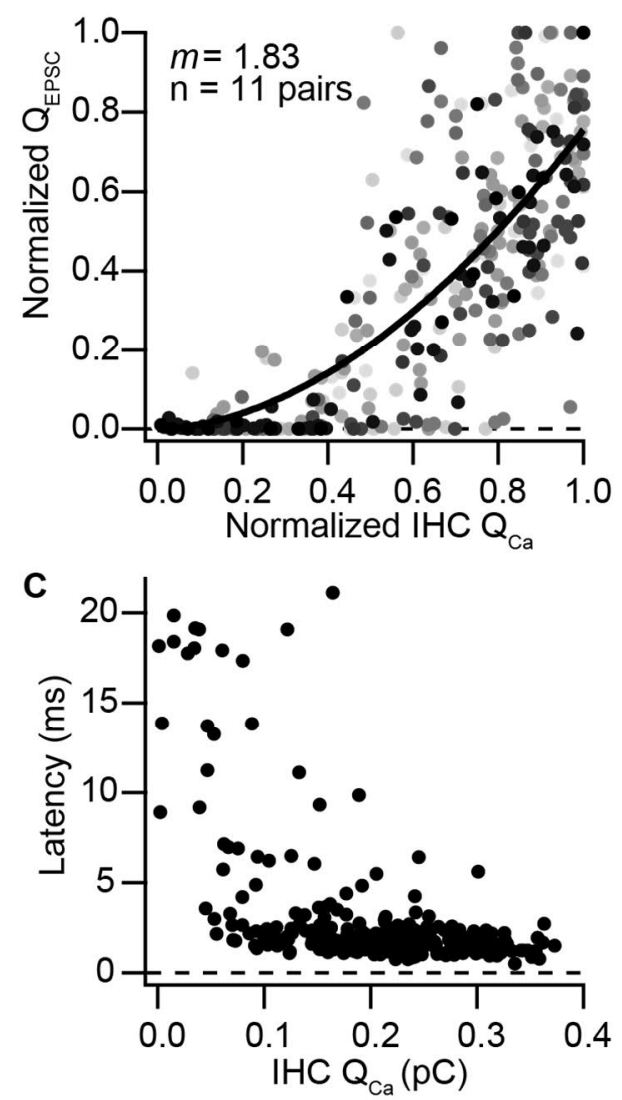

|Figure 8| Few $\mathrm{Ca}^{2+}$ channels control vesicle-stimulus secretion in the physiological range of receptor potentials in IHCs

A. The IHC was depolarized for $2 \mathrm{~ms}$ to different potentials ranging from -58 to $-17 \mathrm{mV}$, triggering different presynaptic $\mathrm{Ca}^{2+}$ currents (blue traces) and the concomitant neurotransmitter release that elicited postsynaptic currents (orange traces). B. Scatter plot of $\mathrm{Ca}^{2+}$ current integrals $\left(\mathrm{Q}_{\mathrm{Ca}}\right)$ versus the corresponding elicited EPSC charge $\left(\mathrm{Q}_{\mathrm{EPSC}}\right)$. The solid line is a least-squares fit of a power of exponent function $\left(\mathrm{Q}_{\mathrm{EPSC}}=\mathrm{a}+\mathrm{b}\left(\mathrm{Q}_{\mathrm{Ca}}\right)^{\mathrm{m}}\right)$ to the data yielding a $\mathrm{Ca}^{2+}$ cooperativity $(\mathrm{m})$ of $1.83(\mathrm{n}=9$ pairs). $\mathrm{C}$. Latency of release (measured from the onset of the stimulus to the onset of the EPSCs) decreased with increasing $\mathrm{Ca}^{2+}$ influx.

Three independent protocols were performed to manipulate the number of open $\mathrm{Ca}^{2+}$ channels. In the first manipulation, we slowly perfused a low concentration $(0.5-2 \mu \mathrm{M})$ of isradipine to gradually reduce the number of $\mathrm{Ca}^{2+}$ channels contributing to the release process during a $5 \mathrm{~ms}$ pulse $\mid$ Figure $7 \mathrm{D}, \mathrm{E} \mid$. This DHP antagonist shifts the $\mathrm{Ca}^{2+}$ channels to an inactivated non-conducting state (Berjukow et al., 2000), but does not affect the single channel current amplitude of the remaining open channels (Hess et al., 1984). As observed during the perfusion of $\mathrm{Zn}^{2+}$, the reduction of $\mathrm{Ca}^{2+}$ influx resulted in the concomitant 
reduction of QEPSC. However, contrary to $\mathrm{Zn}^{2+}$ block, the relationship between QEPSC and $\mathrm{Q}_{\mathrm{Ca}}$ was nearly linear and described by a power function with an exponent of 1.31 |Figure $7 \mathrm{~F} \mid$.

During a second manipulation, we altered the presynaptic $\mathrm{Ca}^{2+}$ influx using $2 \mathrm{~ms}$ depolarizations from $-58 \mathrm{mV}$ to $-17 \mathrm{mV}$ in $2 \mathrm{mV}$ randomized steps |Figure $8 \mathrm{~A} \mid$. This protocol variates $\mathrm{Ca}^{2+}$ influx via changing the open probability, and the single channel current to a lesser extent, over a range covering physiological receptor potentials. These short stimuli were used to avoid synaptic vesicle pool depletion and a possible overlap of $\mathrm{Ca}^{2+}$ domains due to strong $\mathrm{Ca}^{2+}$ influx. Under these conditions, a power of 1.83 suggested that few $\mathrm{Ca}^{2+}$ channels control vesicle fusion in the physiological range of potentials |Figure $8 \mathrm{~B} \mid$. The synaptic delay, measured as the latency between the onset of the stimulus and the onset of the response, shortened with increasing presynaptic $\mathrm{Ca}^{2+} \mid$ Figure $8 \mathrm{C} \mid$.
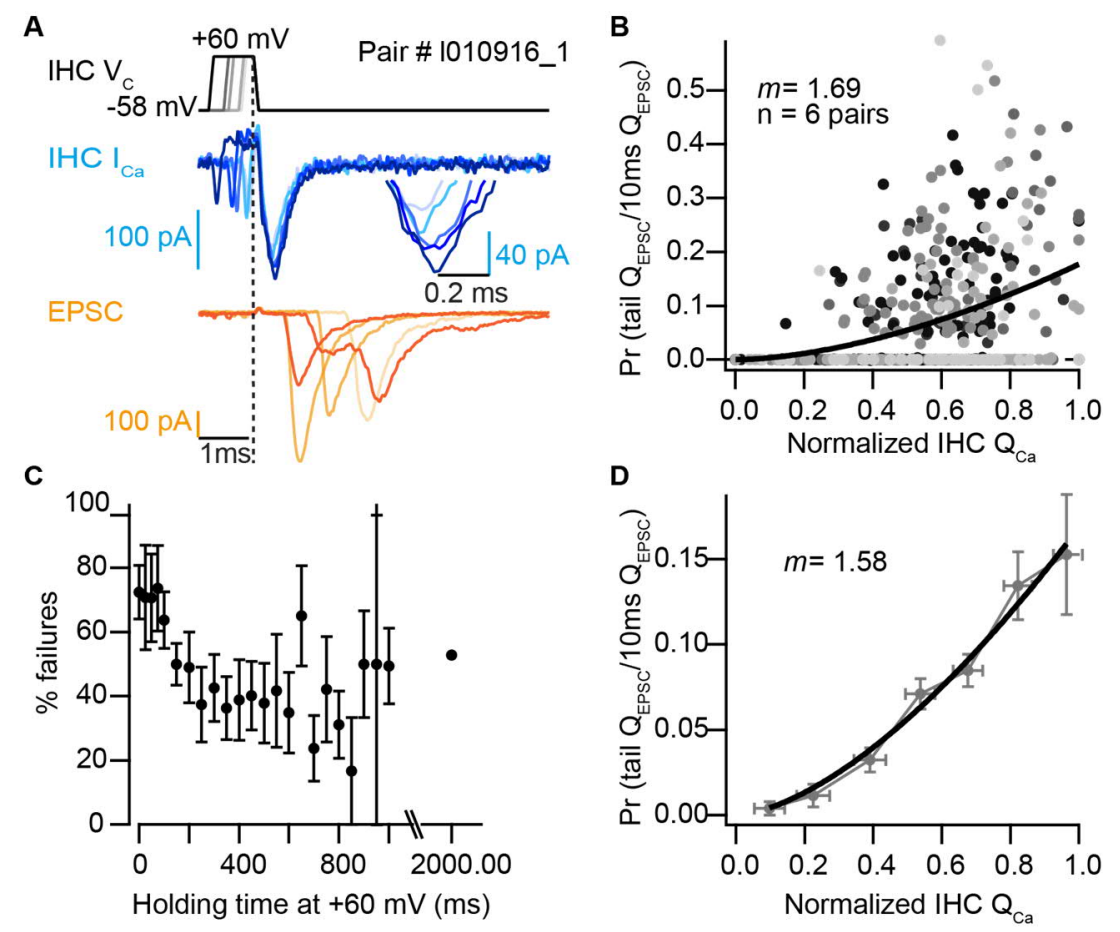

|Figure 9| Neurotransmitter release varies quasi linearly with the number of open $\mathrm{Ca}^{2+}$ channels recruited during $\mathrm{Ca}^{2+}$ tail current protocols

A. Presynaptic voltage steps of increasing duration $(0-2 \mathrm{~ms})$ from $-58 \mathrm{mV}$ to $+60 \mathrm{mV}$ to titrate the number of open $\mathrm{Ca}^{2+}$ channels. Increasing the length of the depolarization increased the amplitude of the $\mathrm{Ca}^{2+}$ tail current (blue traces) and the size of the postsynaptic response. The evoked EPSC was normalized to the responses elicited by $10 \mathrm{~ms}$ voltage steps that fully releases RRP. B. Scatter plot of release probability $\left(\mathrm{P}_{\mathrm{r}}\right.$ : tail $\mathrm{Q}_{\mathrm{EPSC}} / 10$ $\mathrm{ms} \mathrm{Q}_{\mathrm{EPSC}}$ ) vs the normalized presynaptic $\mathrm{Ca}^{2+}$ charge. The solid line is a least-squares fit of a power of exponent function $\left(\mathrm{Q}_{\mathrm{EPSC}}=\mathrm{a}+\mathrm{b}\left(\mathrm{Q}_{\mathrm{C}}\right)^{\mathrm{m}}\right)$ to the data yielding a $\mathrm{Ca}^{2+}$ cooperativity $(m)$ of $1.69(\mathrm{n}=6$ pairs $)$. C. Failures in synaptic transmission were prominent but decreased with the length of the depolarization (data points correspond to mean \pm s.e.m.). D. Power function fit to the binned data (bin size of 0.15 ; datas points are mean \pm s.e.m.) from (B) resulted in a cooperativity of 1.58. 
For the third manipulation, we titrated the number of open $\mathrm{Ca}^{2+}$ channels by eliciting $\mathrm{Ca}^{2+}$ tail currents after depolarizations to $+60 \mathrm{mV}$ of varying durations $(0-2 \mathrm{~ms})$. Increasing the length of the depolarization recruits more open $\mathrm{Ca}^{2+}$ channel and thus, increases the amplitude of the $\mathrm{Ca}^{2+}$ tail current upon repolarization |Figure 9A, inset|. The evoked EPSC was normalized to the responses elicited by $10 \mathrm{~ms}$ voltage steps that fully releases the RRP. The resulting release probability $\left(\mathrm{P}_{\mathrm{r}}\right)$ was plotted against the normalized IHC Q $\mathrm{Q}_{\mathrm{Ca}}$ Figure 9B|. The relationship was described by a power function with a coefficient of 1.69 . Synaptic transmission had a considerable number of failures that decreased with the length of the depolarization, although they were still present even at maximum $\mathrm{Ca}^{2+}$ influx $\mid$ Figure 9B, C|. Therefore, a power function was also fitted to the binned data (bin size of 0.15) |Figure 9D|. The power fit yielded an apparent cooperativity of 1.58 .

Taken together, these findings validate that physiological sound encoding relies on the close coupling of few $\mathrm{Ca}^{2+}$ channels to control fusion of a given vesicle, and support the hypothesis of $\mathrm{Ca}^{2+}$ nanodomain-like control of vesicle release.

\section{Discussion}

Much of the information regarding neurotransmitter release from mouse IHCs has often been based on summing over all synapses of a given IHC and has been acquired in unphysiological recording conditions. Since exocytosis is a $\mathrm{Ca}^{2+}$-, temperature- and activitydependent process, we investigated the first synapse of the auditory pathway under nearphysiological conditions. We performed paired pre- and postsynaptic recordings from single IHC synapses using protocols that provide the opportunity for comparison to in vivo recordings of spontaneous and sound-evoked spiking in SGNs. We analyzed synapse properties in terms of spontaneous activity, vesicle pool dynamics, as well as dynamic range and $\mathrm{Ca}^{2+}$-dependence of release. We found that SGNs contacting the pillar side of the IHC exhibited a higher spontaneous rate of EPSCs compared to synpases contacting the modiolar side. High SR synapses had larger sponEPSCs that tended to assume a more compact waveform (i.e., without inflections and faster kinetics in the rise and decay phases) in contrast to low SR synapses. In terms of evoked synaptic transmission, high SR synapses were characterized by lower voltage-thresholds. Although not significant in our small data set, high SR synapses had on average shorter latencies of evoked neurotransmitter release, higher onset rates and a slightly faster recovery from RRP depletion compared to low SR synapses. The RRP size and rates of sustained exocytosis, as well as the dynamic range of 
release, were comparable regardless the SR of the synapses. Furthermore, our study corroborates that a $\mathrm{Ca}^{2+}$-nanodomain-like control of exocytosis operates at IHCs synapses under near physiological conditions.

\section{Pillar synapses have higher rates of spontaneous release}

In order to recapitulate synaptic transmission in the absence of sound stimulation, we aimed to hold the IHC at their tentative resting potential (around $-58 \mathrm{mV}$, Johnson, 2015). The IHC resting potential is set by the endolymph, perilymph and intracellular ionic compositions (Sewell, 1984), and by the interplay between the MET channels (Johnson, 2015) and several voltage-dependent $\mathrm{K}^{+}$channels (Kros et al., 1998; Marcotti et al., 2003, 2004; Oliver et al., 2003). Since Cav1.3 $\mathrm{Ca}^{2+}$-channels activate at negative voltages (-65 to $-45 \mathrm{mV}$, (Koschak et al., 2001; Platzer et al., 2000; Xu and Lipscombe, 2001)), their open probability at the IHC resting potential might be sufficient to trigger exocytosis. Indeed, spontaneous SGN firing depends on such $\mathrm{Ca}^{2+}$ channel openings (Robertson and Paki, 2002). Additionally, this depolarized resting potential renders the IHC in a permanently facilitated state that may mediate a faster $\mathrm{Ca}^{2+}$ current activation and shorter latency of release (Cho and von Gersdorff, 2012; Goutman and Glowatzki, 2011). The concentration of resting $\mathrm{Ca}^{2+}$ can already have an effect on $\mathrm{Ca}^{2+}$ dependent processes such as vesicle priming and recruitment (Awatramani et al., 2005). Interestingly, the rate of spontaneous firing of SGNs with similar characteristic frequency (i.e. potentially synapsing on the same IHC) ranges from zero to 120 spikes/s (Taberner and Liberman, 2005). This variability in the rate of spontaneous firing among SGNs has been attributed, at least in part, to differences in the voltage-dependent activation of $\mathrm{Ca}^{2+}$ influx at their presynaptic active zones of IHCs (Frank et al., 2009; Ohn et al., 2016).

Under our experimental conditions, the rates of spontaneous transmission also varied substantially, ranging from 0 up to $18 \mathrm{EPSC} / \mathrm{s}$. Our SRs do compare to the ones recorded ex vivo from the afferent bouton of rats of a similar age group (p15-p17, Wu et al., 2016). Assuming each EPSC will trigger one action potential in the SGN for low EPSC rates (Rutherford et al., 2012), the maximum spontaneous EPSC rate found in this study would predict spontaneous firing rates 3 times smaller than those recorded in vivo from single ANFs of p14-p21 mice (60 spikes/s; Wong et al., 2013) and at least 6 times lower than those recorded from 16-17 weeks old mice (up to $120 \mathrm{spikes/s} \mathrm{in} \mathrm{mouse,} \mathrm{Taberner} \mathrm{and} \mathrm{Liberman,}$ 2005). Additionally, multiphasic EPSCs may have a lower chance to trigger an action potential in the postsynapse, further segreggating the spontaneous spikes rates between low 
and high ANFs (Grant et al., 2010). This might hold true in 4 out of the 13 low SR synapses whose average amplitude of sponEPSC fell below $50 \mathrm{pA}$, the low rheobase of spike generation (Rutherford et al., 2012). The pronounced difference between in vivo and ex vivo could be attributed to the age group, since developmental changes lead to the gradual recruitment of high SRs fibers in older animals [seen in in vivo data from mice, (Wong et al., 2013), kittens (Romand, 1984; Walsh and McGee, 1987) and in vitro data from rats (Wu et al., 2016)]. Additionally, we cannot exclude a sampling bias accounted for the lower probability of encountering a high SR synapse due to the fact that about $65 \%$ of the mouse fibers in vivo have a SRs $<20$ spikes/s (Taberner and Liberman, 2005). Finally, there can be differences in the recording conditions despite our efforts to mimic the IHC's in vivo environment in terms of resting potential, composition of the artificial perilymph and temperature.

However, the fraction of synapses with a release rate higher than $1 \mathrm{EPSC} / \mathrm{s}$ ("high spontaneous rate", following the definition based on ANF firing by Taberner and Liberman, 2005) compares to the values obtained for spontaneous mouse ANF firing in vivo, where about $25 \%$ of the ANFs had a SR $>1$ spike/s (Taberner and Liberman, 2005). In our recordings, high SR synapses were found exclusively on the side of the IHC facing the inner pillar cells ("pillar side"), agreeing with findings from in vivo labelling of physiologically characterized single auditory fibers in cats (Liberman, 1982). Yet, not all the synapses of the pillar face had high frequencies of spontaneous release. It is important to point out that our modiolar/pillar classification is based on the side where the postsynaptic pipette was positioned, and it is therefore less precise as in other studies (Frank et al., 2009; Liberman et al., 2011; Ohn et al., 2016). Moreover, other functional readouts of IHC synapses also support an overall pillar-modiolar gradient of synaptic properties rather than their strict segregation (Ohn et al., 2016).

\section{Distinct release modes at low and high SR synapses}

To estimate the numbers of vesicles released in a single active zone during stimulation, we used the average charge of the spontaneous EPSCs obtained for each paired recording. This assumes that each sponEPSC corresponds to a unitary release event ("univesicular mode of release" (Chapochnikov et al., 2014)). In contrast to the hypothesis that coordinated multivesicular release explains the large variability of amplitude and shape of spontaneous EPSCs (Glowatzki and Fuchs, 2002), Chapochnikov et al. (2014) proposed an alternative model wherein neurotransmitter release from a single vesicle through a dynamic fusion 
accounts for the observed EPSC heterogeneity. Recently, two independent studies using low noise cell-attached capacitance measurements and postsynaptic bouton recordings further supported the hypothesis that the univesicular mode of release prevails at IHC synapses (Grabner and Moser, 2018; Huang and Moser, 2018). Our finding of similar average charge of sponEPSCs between different pairs, despite the high variability in their amplitudes, adds support to the hypothesis of univesicular mode of release.

Similar to previous studies (Glowatzki and Fuchs, 2002; Grant et al., 2010), we found a high variability in the modes of release between synapses, with some synapses exhibiting no monophasic EPSCs. The dissimilarities in the percentage of multiphasic EPSCs in low and high SR fibers could arise from variability in the fusion pore dynamics on the way to vesicle fusion. We hypothesize that flickering of the fusion pore, instead of a fusion pore opening followed directly to full vesicle collapse, might be favored in low SR (and modiolar) synapses, leading to a higher percentage of multiphasic EPSCs.

In general, high SR synapses also had shorter synaptic delay times, significantly lower thresholds of voltage-triggered release (evaluated by the EPSC $\mathrm{Q}_{10}$ ) and a more hyperpolarized voltage of half-maximal release. These characteristics might originate from a more hyperpolarized operating range of $\mathrm{Ca}^{2+}$ influx and, consequently, transmitter release of pillar AZs (Ohn et al., 2016). Recent evidence supports the idea that longer duration of $\mathrm{Ca}^{2+}$ influx at the release site promotes full-collapse fusion (Huang and Moser, 2018). Subsequently, it is tempting to speculate that heterogeneity in the gating of $\mathrm{Ca}^{2+}$ channels along the modiolar/pillar axis partly accounts for the variability of spontaneous EPSCs waveforms and SRs between synapses. Possible factors that could set distinct biophysical properties of the $\mathrm{Ca}^{2+}$ channels -comprising but not limited to their voltage sensitivityinclude different splice variants, preferential presence of specific auxiliary subunits and modulation by interacting proteins (Hoppa et al., 2012; Jean et al., 2018; Neef et al., 2009; Ohn et al., 2016; Scharinger et al., 2015).

$\mathrm{Ca}^{2+}$ channel density and their topography relative to the vesicular release sites -which, if different, could go along with heterogeneity of stimulus-secretion coupling between synapses- might be alternative and/or complementary mechanisms to regulate kinetics of vesicle release. Indeed, modiolar AZs are, on average, larger, with a greater number of $\mathrm{Ca}^{2+}$ channels and maximal synaptic $\mathrm{Ca}^{2+}$ influx (Liberman et al., 2011; Ohn et al., 2016). How do modiolar synapses with bigger $\mathrm{Ca}^{2+}$ channel clusters have lower SRs and sensitivity to sound? Neurotransmitter release properties depends on two parameters acting in concert, the 
size of the RRP and the release probability of these vesicles. Contrary to previous morphological estimates of the RRP size from low and high SR synapses (Kantardzhieva et al., 2013), our physiological estimates from a relatively small dataset do not show a clear divergence in RRP size between the low and high SR synapses. However, our current estimates of RRP come only from synapses positioned in the pillar face of the IHC. Whether modiolar synapses migth hold indeed a larger RRP due to their larger synaptic size (Wittig and Parsons, 2008), remains to be determined using forward masking recordings from modiolar boutons. In addition, multiple ribbons per contacting bouton have also been reported to occur predominantly in modiolar synapses (Michanski et al., 2019), increasing the possibility of observing larger RRPs at modiolar/low SR synapses.

Regarding release probability, multiple factors govern it: the number of $\mathrm{Ca}^{2+}$ channels (Gratz et al., 2019; Holderith et al., 2012; Scimemi and Diamond, 2012; Sheng et al., 2012; Wong et al., 2013), the spatial coupling of the $\mathrm{Ca}^{2+}$ channels to the vesicular release site (Eggermann et al., 2012; Moser et al., 2019) and the fusion competence of the individual synaptic vesicles (Klenchin and Martin, 2000). Using STED microscopy, Neef and colleagues observed variable length but similar width of the $\mathrm{Ca}^{2+}$ channel clusters between active zones (Neef et al., 2018). They postulated a conserved topography of $\mathrm{Ca}^{2+}$ channels and coupling distance- in IHC active zones regardless of their size (Neef et al., 2018). In the present study, the apparent $\mathrm{Ca}^{2+}$ cooperativity was assessed mostly on synapses from the modiolar face of the IHC, hindering the evaluation of heterogeneity along the modiolar-pillar axis in the $\mathrm{Ca}^{2+}$ dependence of release. Interestingly, high SR synapses contacted IHC with a more hyperpolarized activation potential of whole cell $\mathrm{Ca}^{2+}$ influx. This poses the question if part of the diversity in the SGN response properties, in addition to the heterogeneity of $\mathrm{Ca}^{2+}$ influx among AZs within an IHC, comes from overall differences in the voltage dependence of activation or differences in the resting potential between IHCs. Future studies combining the readout of neurotransmitter release with $\mathrm{AZ} \mathrm{Ca}^{2+}$ imaging or $\mathrm{Ca}^{2+}$ uncaging will help to understand the impact of the number and/or the coupling distance of $\mathrm{Ca}^{2+}$ channels in the diversity of response properties of IHC ribbon synapses.

\section{Synaptic vesicle pool dynamics at individual active zones}

In response to a constant sound stimulus, auditory nerve fibers recorded in vivo show a maximum onset spike rate that declines rapidly (Taberner and Liberman, 2005). The peak firing rate is thought to reflect the release of the standing RRP (release sites occupied by synaptic vesicles (Oesch and Diamond, 2011; Pangršič et al., 2012). The adapted firing rate 
is thought to reflect synaptic vesicle replenishment [reviewed in (Pangršič et al., 2012; Rutherford and Moser, 2016)]. During the masker stimulus in our recordings, neurotransmitter release at individual synapses was also characterized by an initial high rate of $\sim 2400$ vesicles/s, followed by a slower sustained rate of $\sim 445$ vesicles/s. We estimated a standing RRP of $\sim 12$ vesicles (and a total RRP of 18 release slots based on the model), fusing with a time constant of $6.47 \mathrm{~ms}$. Our estimated standing RRP compares to prior results obtained using capacitance measurements of murine IHCs (Beutner et al., 2001; Moser and Beutner, 2000; Pangrsic et al., 2010), in which RRP sizes and release rates were calculated with a conversion factor of $45 \mathrm{aF}$ per synaptic vesicle and an average of 12 synapses per IHC, and to the ones previously determined using paired recordings in rats (Goutman and Glowatzki, 2007). These numbers also fit well with electron microscope estimates of 12-16 vesicles in close proximity to the plasma membrane $(<50 \mathrm{~nm})$ (Chakrabarti et al., 2018; Khimich et al., 2005).

The initial and sustained rates of release were not statistically different between low and high SR fibers. However, the highest release rates observed in our relatively small sample dataset were uniquely found from high SR synapses, comparable to reports from ANFs recorded in vivo (Buran et al., 2010; Taberner and Liberman, 2005). Based on the number of synaptic vesicle release slots $\left(\mathrm{N}_{\text {slots }}\right)$ and the fusion rate constant estimated from the model fit, onset release could occur at a maximal rate of 3384 vesicles/s if all the release sites are occupied with release ready vesicles at stimulus onset. Thus, the status of the synapse will critically influence the observed release rates during stimulation (Cho and von Gersdorff, 2012; Goutman and Glowatzki, 2011).

\section{Nanodomain control of exocytosis at murine IHCs}

Because of the technically challenging recordings from the postsynaptic bouton of mammalian SGNs, most of the studies tested the IHC $\mathrm{Ca}^{2+}$ dependence of exocytosis using whole cell capacitance recordings that measured the sum of several active zones of one IHC (Brandt et al., 2005; Johnson et al., 2017; Wong et al., 2014). Only one study so far implemented simultaneous pre- and postsynaptic paired recordings from rats, nonetheless before the onset of hearing (Goutman and Glowatzki, 2007), when developmental synaptical refinements have not occurred yet (Johnson et al., 2005, 2007, 2008, 2009; Wong et al., 2014). In addition, the stimulus duration used in most of the cases typically exceeded the phase of initial release, partially depleting the RRP and including vesicle replenishment, 
which complicates the interpretation of the results (e.g. (Wong et al., 2014)). Overall, all these factors might explain the discrepancy in experimental findings among different studies.

Using simultaneous patch clamp recordings from murine IHCs and their postsynaptic SGN terminals after the onset of hearing, the present work provides strong and most direct support that a $\mathrm{Ca}^{2+}$ nanodomain-like control of synaptic vesicle fusion operates during physiological sound encoding. The high temporal resolution of this technique allowed us to use short stimulation protocols avoiding depletion of RRP, prominent vesicle replenishment and possible overlap of $\mathrm{Ca}^{2+}$ domains due to strong $\mathrm{Ca}^{2+}$ influx. Three independent manipulations to titrate the number of open $\mathrm{Ca}^{2+}$ channels converged in the result of a nearlinear relationship between $\mathrm{Ca}^{2+}$ influx and vesicle fusion at single ribbon synapses, similar to previous reports mentioned before. Nanodomain control of release ensures rapid and reliable release of neurotransmitter upon stimulation and thus preserves the temporal precision of the synapses (Neher, 1998). 
Chapter 2 


\section{Chapter 3 The synaptic ribbon is critical for sound encoding at high rates and with temporal precision}

Philippe Jean†, David Lopez de la Morena†, Susann Michanski†, Lina María Jaime Tobón†, Rituparna Chakrabarti, Maria Magdalena Picher, Jakob Neef, SangYong Jung, Mehmet Gültas, Stephan Maxeiner, Andreas Neef*, Carolin Wichmann*, Nicola Strenzke*, Chad Grabner*, Tobias Moser*

$\dagger$ These authors contributed equally to the work eLife 2018;7:e29275.

DOI: https://doi.org/10.7554/eLife.29275

Appendix 9.1

Detailed author contribution of Lina María Jaime Tobón

- Experimental work and analysis:

- Performed patch-clamp capacitance measurements (Fig.7D-H)

- Data curation, Formal analysis, Investigation, Visualization, Writing —original draft, Writing — review and editing

Copyright notice:

This material is reprinted by permission from eLife Ltd. open access journals in accordance to their copyright and licensing regulations (CC BY license 4.0 https://creativecommons.org/licenses/by/4.0/legalcode). ( $)$ owned by the authors, 2018. 
Chapter 3 


\title{
Chapter 4 Endophilin-A regulates presynaptic $\mathbf{C a}^{2+}$
} influx and synaptic vesicle recycling in auditory hair cells Jana Kroll*, Lina M Jaime Tobón*, Christian Vogl*, Jakob Neef, Ilona Kondratiuk, Melanie König, Nicola Strenzke, Carolin Wichmann, Ira Milosevic, Tobias Moser

\author{
*These authors contributed equally to the work \\ EMBO J (2019)38:e100116 \\ DOI: https://doi.org/10.15252/embj.2018100116
}

Appendix 9.2

Detailed author contribution of Lina María Jaime Tobón

- Experimental work and analysis:

- Contributed to single-cell real-time PCR (Fig. 1A,B)

- Performed immunohistochemistry and analysis (Fig. 3A,A',D,D')

- Performed single-cell patch-clamp capacitance measurements and analysis (Fig.4, 6)

$\circ$ Analysis of $\mathrm{Ca}^{2+}$ inactivation (Fig. 2B,B')

- Prepared the manuscript with contributions of all the authors.

Copyright notice:

This material is reprinted by permission from EMBO Press in accordance to their copyright and licensing regulations. Reprinted by permission from Wiley-VCH Verlag $\mathrm{GmbH} \& \mathrm{Co}$. KGaA: (c) owned by the authors, 2019. 
Chapter 4 


\section{Chapter 5 General discussion}

Inner hair cells (IHCs) and their unconventional synapses have fascinated researchers over the years. In vivo recordings from mammalian auditory nerve fibers (ANFs) revealed impressive performance of synaptic sound encoding. Sound stimulation evoked, within submilliseconds, a highly synchronized onset response with a jitter lower than $1 \mathrm{~ms}$ (Buran et al., 2010). Even though adaptation to lower firing rates occurred after few milliseconds, transmission indefatigably sustained high firing rates up to hundreds spikes/s with sound stimulation. Strikingly, ANFs had spontaneous firing rates (SR) that ranged below 0.5 spikes/s to more than 100 spikes/s depending on the species [up to 120 spikes/s for rat, cat and mouse (Barbary, 1991; Kiang et al., 1965; Liberman, 1978; Taberner and Liberman, 2005), up to 140 for guinea pigs and gerbils (Evans, 1972; Schmiedt, 1989), and up to 200 spikes/s for chinchilla (Temchin et al., 2008)]. Interestingly, low and high SR fibers encode sound differently. For example, sound threshold (Ohlemiller et al., 1991; Sumner and Palmer, 2012; Taberner and Liberman, 2005; Tsuji and Liberman, 1997; Winter et al., 1990) and the spike rate variation within a range of sound intensities (termed dynamic range) differed between low and high SR fibers (Ohlemiller et al., 1991; Sachs and Abbas, 1974; Taberner and Liberman, 2005; Winter et al., 1990). Since the SR was uncorrelated to the sound frequency at which each fiber achieved the maximum sensitivity to sound (characteristic frequency), it was hypothesized that both low and high SR ANFs contact the same IHC (Johnson and Kiang, 1976). A plethora of techniques has been used to understand how IHCs accomplish fast and indefatigable release of neurotransmitter, and which mechanisms establish the diversity of the ANF responses to stimulation. In this thesis, I provided further insight into the molecular physiology of sound encoding.

First, I studied synaptic transmission at individual murine IHC afferent synpases employing paired IHC-bouton patch clamp recordings in near physiological conditions. Boutons contacting the pillar side of the IHC showed higher rates of spontaneous EPSCs. The spontaneous EPSCs from high SR synapses tended to assume more compact waveforms and were characterized by larger amplitudes yet similar charges. During voltage step stimulations, synaptic transmission followed an initial release rate of 2400 vesicles/s that depleted with a time constant of $6.47 \mathrm{~ms}$ to a sustained release rate around 445 vesicles/s. The latency of the evoked release was around $1.27 \mathrm{~ms}$. High SR synapses had significantly lower voltage thresholds of release with a tendency to have shorter synaptic delays and faster 
recovery from RRP depletion. Furthermore, this study corroborates that a $\mathrm{Ca}^{2+}$-nanodomainlike control of exocytosis operates at IHCs synapses under near physiological conditions.

Second, collaborators and I studied synaptic transmission in ribbonless IHC synapses from mice lacking RIBEYE. Structurally, these synapses contained several small active zones opposing each postsynaptic density instead of one active zone. In vivo ANFs recordings revealed an impaired synaptic transmission, characterized by lower spontaneous and evoked firing rates, lower temporal precision and a slower recovery from adaptation. $\mathrm{Ca}^{2+}$ imaging of individual active zones showed that the $\mathrm{Ca}^{2+}$ channels required more depolarized potentials to activate. This resulted in reduced exocytosis to weak depolarizations assessed by perforated patch-clamp recordings. We postulated a role of the ribbon in synaptic vesicle replenishment and $\mathrm{Ca}^{2+}$ channel regulation.

Third, collaborators and I studied the role of endophilin-A1-3, endocytic adaptor proteins, in IHCs. Perforated patch-clamp recordings from organotypic cultures and from explanted organs of Corti revealed lower $\mathrm{Ca}^{2+}$ influx, impaired sustained exocytosis and slower endocytic membrane retrieval in Endophilin-A-deficient IHCs. At the ultrastructural level, the IHC active zones had lower counts of synaptic vesicles, but increased numbers of coated structures and endosome-like vacuoles. In addition, we postulated a molecular interaction between endophilin-A1 and otoferlin based on co-immunoprecipitation. We proposed a positive role of endophilin-A in the modulation of $\mathrm{Ca}^{2+}$ channels, and in synaptic vesicle recycling, likely via coupling of exo- and endocytosis, membrane retrieval, synaptic vesicle uncoating and reformation.

In this general discussion, I will start by briefly reviewing the advances made by different techniques in understanding synaptic vesicle pool dynamics. I will then discuss the mechanisms underlying the temporal acuity and indefatigable release of mammalian IHCs, covering vesicle pool dynamics, mechanisms for synaptic vesicle reformation and replenishment, and properties and distribution of $\mathrm{Ca}^{2+}$ channels. I will finish by briefly summarizing the mechanisms mediating synaptic heterogeneity and its relation to the diversity in the response properties of SGNs.

\section{Techniques to study exocytosis at IHCs}

Most of the estimates of the presynaptic function of IHCs originated from biophysical studies using whole-cell patch clamp recordings with capacitance measurements (Beutner and 
Moser, 2001; Brandt et al., 2005; Johnson et al., 2005; Moser and Beutner, 2000; Pangrsic et al., 2010) or with $\mathrm{Ca}^{2+}$ imaging (Frank et al., 2009; Ohn et al., 2016), fluorescence membrane imaging (Griesinger et al., 2005), and from models fitted to in vivo responses from ANFs (Buran et al., 2010; Frank et al., 2010; Jean et al., 2018; Jung et al., 2015; Peterson et al., 2014). Current estimates fall in the range of 1800-10200 vesicles/s for onset release rate and 177-679 vesicles/s for sustained (adapted) release rates per active zone, with an RRP size between 4 to 45 vesicles that depletes with a time constant of 3 to $54 \mathrm{~ms}$. The wide ranges of these numbers might reflect different animal models, experimental protocols, recording conditions, differences in analysis and/or assumptions in the models. In addition, while still being powerful, each method has drawbacks. Whole-cell capacitance measurements, for instance, sample exocytosis occurring at the entire set of active zones as well as extrasynaptically in one IHC (Fuchs et al., 2003; Pangršič et al., 2015). Furthermore, the signal to noise of the technique (typically between 1-10 fF, Lindau and Neher, 1988) is insufficient to study unitary fusion events which produce capacitance increments as small as $40 \mathrm{aF}$, unless variance analysis (Neef et al., 2007) or cell-attached capacitance measurements (Grabner and Moser, 2018) is used.

Modeling of the spike responses from ANFs relies on the assumption of several parameters that are still under debate for IHC afferent synapses, including the contribution of AMPA receptor desensitization to the steady state postsynaptic response (Goutman, 2017; Goutman and Glowatzki, 2007), the relation between synaptic input and spike generation (Grant et al., 2010; Rutherford et al., 2012), particularly during stimulation, and multivesicular vs. univesicular mode of vesicle release (Chapochnikov et al., 2014; Glowatzki and Fuchs, 2002; Grabner and Moser, 2018; Grant et al., 2010; Graydon et al., 2011; Huang and Moser, 2018; Matthews and Sterling, 2008; Rudolph et al., 2015). In addition, while in vivo recordings bring the advantage of an unperturbed milieu for the IHCs, interpretation of the response needs to consider factors upstream of the depolarization of the IHC (e.g., basilar membrane displacement, stimulus amplification by the $\mathrm{OHCs}$, mechanotransduction process at the IHC stereocilia, receptor potential dynamics). On top, few in vivo experiments have offered a correlation between the SGN response properties and the fiber's relative position to the IHC or the synaptic ultrastructure (Liberman, 1982; Merchan-Perez and Liberman, 1996). This is particularly true for mice, for which the challenging morphological description of functionally characterized ANFs has not yet been performed. This last point acquires great importance when considering the reported functional and morphological presynaptic heterogeneity across active zones of one IHC [chapter 2 (Frank et al., 2009; Jean et al., 2019; 
Kantardzhieva et al., 2013; Merchan-Perez and Liberman, 1996; Michanski et al., 2019; Ohn et al., 2016)].

The first in vitro whole-cell patch-clamp recording from the bouton of a mammalian SGN offered the temporal resolution to record individual release events of a single active zone (Glowatzki and Fuchs, 2002). These experiments allowed to experimentally manipulate the synapse using pharmacological approaches. Key features about the ribbon synapse were unraveled, including the high variability of EPSC amplitudes -ranging from 20 to $800 \mathrm{pA}-$ and of EPSC waveforms - monophasic and multiphasic- in individual synapses (Glowatzki and Fuchs, 2002). Further studies showed that the rate of EPSC discharge and the proportion of monophasic EPSCs vary greatly between boutons (Grant et al., 2010), findings that might contribute to the diversity of the ANFs response properties in vivo (for a review see Heil and Peterson, 2015). Specific and precise stimulation of the IHC was not possible in the above studies, and thus, stimulus-secretion dependence and synaptic vesicle pool dynamics remained open questions.

Paired IHC and bouton whole-cell patch-clamp recordings from rats provided crucial information regarding synaptic transmission during stimulation. The time course of neurotransmitter release followed an initial rate of more than $1000 \mathrm{EPSC} / \mathrm{s}$ that adapted to $43 \mathrm{EPSC} / \mathrm{s}$ within $3 \mathrm{~ms}$ (Goutman and Glowatzki, 2007). Under the assumption that the variable size of single EPSCs is caused by multivesicular release, the authors estimated an onset release rate over 7000 vesicles/s, a sustained rate of about 300 vesicles/s, and RRP size of 12 vesicles. Due to their unphysiological recordings conditions in terms of temperature (room temperature), IHC resting potential (around $-80 \mathrm{mV}$ ) and composition of the extracellular medium (HEPES-based), the estimates might not truly compare to sound encoding in vivo. Furthermore, recordings were performed previous to the onset of hearing, before some developmental refinements have taken place in the synapse [e.g. tightening of the coupling of $\mathrm{Ca}^{2+}$ influx to exocytosis (Johnson et al., 2005; Wong et al., 2014), reduction of the proportion of multiphasic EPSCs (Grant et al., 2010), emergence of high spontaneous rate fibers (Wong et al., 2013; Wu et al., 2016)]. Finally, distinction of the bouton's position and spontaneous activity was not addressed in these studies.

In the present thesis, I established paired pre- and post-synaptic recordings in near physiological conditions to understand the response properties of individual ribbon synapses from mice after the onset of hearing. The recordings conditions are of utmost importance given that the physiological state and environment of the IHC critically shapes synaptic 
transmission. $i$ ) The relatively depolarized IHC resting potential (around $-58 \mathrm{mV}$, Johnson, 2015) sets the synapses in a constant facilitated mode (Cho and von Gersdorff, 2012; Goutman and Glowatzki, 2011); this is attributed to $\mathrm{Ca}^{2+}$ influx mediated by the stochastic opening of the $\mathrm{Ca}_{\mathrm{v}} 1.3$ channels which have a lower voltage of activation compared to other $\mathrm{Ca}^{2+}$ channels (-65 to $-45 \mathrm{mV}$, (Koschak et al., 2001; Platzer et al., 2000; Xu and Lipscombe, 2001)). ii) Temperature influences both $\mathrm{Ca}^{2+}$ influx and exocytosis [(Kushmerick et al., 2006; van Lunteren et al., 1993; Micheva and Smith, 2005; Renden and von Gersdorff, 2007); also in IHCs (Nouvian, 2007)]. iii) The presence and concentration of exogenously added $\mathrm{Ca}^{2+}$ buffers in the cell affects the release of synaptic vesicle pools (Adler et al., 1991; Moser and Beutner, 2000). iv) Proton-mediated modulation of $\mathrm{Ca}^{2+}$ channels might be affected by the presence of strong extracellular proton buffers such HEPES (Chen et al., 1996; Cho and von Gersdorff, 2014; DeVries, 2001; Palmer et al., 2003; Vincent et al., 2018). In an attempt to emulate the physiological state and milieu of IHCs, paired recordings were performed at $33-37^{\circ} \mathrm{C}$ with an extracellular solution that mimics the perilymph [including $25 \mathrm{mM}$ sodium bicarbonate and $1.3 \mathrm{mM} \mathrm{CaCl}_{2}$; (Wangemann and Schacht, 1996)]. IHCs were patched at $-58 \mathrm{mV}$ using perforated patch-clamp technique to better preserve the intracellular composition. Under these conditions, synaptic transmission followed an initial release rate of 2400 vesicles/s that depleted with a time constant of 6.47 $\mathrm{ms}$ to a sustained release rate around 445 vesicles/s. The difference in the release rates from the ones determined by (Goutman and Glowatzki, 2007) could originate from their assumption that single EPSCs represent multivesicular release. They deconvolved the response using an average EPSC waveform obtained from non-overlapping EPSCs recorded during stimulation. In the work presented here, I used spontaneous EPSCs recorded in absence of stimulation as the unitary event for each pair. This relies on the assumption that spontaneous EPSCs correspond to the release event of single vesicles (Chapochnikov et al., 2014; Huang and Moser, 2018).

Distinction between boutons with high $(\geq 1 \mathrm{EPSC} / \mathrm{s})$ or low $(<1 \mathrm{EPSC} / \mathrm{s})$ was also made in this thesis. The relatively small dataset already suggests differences in release rates according to the basal activity of the synapse. Further experiments to expand the dataset are required to draw stronger conclusions. Additionally, it was typically not possible to obtain two boutons from the same IHC, limiting conclusions regarding the functional heterogeneity across active zones of one IHC. Fluorescence imaging methods could offer a complementary approach to simultaneously monitor exocytosis at several active zones (for review see Kavalali and Jorgensen, 2014). However, genetically encoded exocytosis sensors, such as 
pHluorin, mOrange2 and pHtomate, reported a low signal-to-noise ratio in IHCs, making them unsuitable to monitor synaptic activity in these cells (Neef et al., 2014; personal communication with Özge Demet Özcete). Future efforts to establish further optical methods could advance our understanding of synaptic heterogeneity.

\section{Inner hair cell synaptic vesicle pool dynamics}

\section{Spontaneous activity}

ANFs fire action potentials in vivo in the absence of sound (Sewell, 1984; Taberner and Liberman, 2005). Such spontaneous activity has also been observed in EPSC recordings from the postsynaptic SGN bouton from explanted organs of Corti (Chapochnikov et al., 2014; Glowatzki and Fuchs, 2002; Grant et al., 2010; Wu et al., 2016). These observations, in combination with the reported dependence of the spontaneous activity on the activity of $\mathrm{Ca}^{2+}$ channels (Robertson and Paki, 2002) and the irregular ocurrance of the events (review by Kim et al., 2013), suggested that spontaneous SGN firing reflects the stochastic spontaneous, but $\mathrm{Ca}^{2+}$-driven, release of neurotransmitter from the presynaptic IHC. Loose bouton patch extracellular recordings provided further evidence that the ANF spontaneous firing properties observed in vivo derive from presynaptic mechanisms of vesicular release (Wu et al., 2016).

During paired IHC-bouton recordings in near physiological conditions performed in this thesis (chapter 2), spontaneous release of neurotransmitter occurred between 0 and 18 EPSCs/s, with $75 \%$ of the synapses having low SR (below 1 EPSCs/s; following the high and low SR classification proposed in mice by Taberner and Liberman, 2005). These results are comparable to results from loose-patch clamp recordings of ANFs from explanted rat cochleae (Wu et al., 2016). Based on the low rheobase for spike generation determined using bouton patch clamp recordings (around $50 \mathrm{pA}$; Rutherford et al., 2012) and the high reliability of EPSPs to trigger action potentials in vivo (around 12\% failure rate; Siegel, 1992), the EPSC rates can be translated into spike rates. This would suggest inferior spontaneous spike rates than those observed in vivo of up to $60 \mathrm{spikes} / \mathrm{s}$ in mice after hearing onset (Wong et al., 2013) and up to 120 spikes/s in 16-17 weeks old mice (Taberner and Liberman, 2005). The discrepancy between rates observed in vitro and in vivo could be attributed to a sampling bias due to lower percentage of fibers with SRs $>20$ spikes/s (around $35 \%$ in mice; Taberner and Liberman, 2005). However, the range of SRs should not be underrepresented by the sampling region of the organ of Corti since SRs do not variate with 
the ANFs characteristic frequency in rat and mice (Barbary, 1991; Taberner and Liberman, 2005). Effects of the recording conditions, such as variations of the IHC resting potential, composition of the artificial perilymph and/or fluctuations in the temperature cannot be excluded.

Dual afferent recordings provided the first direct evidence that low and high discharge rate fibers contact the same IHC (Wu et al., 2016). In line with the hypothesis that high SR fibers contact predominantly the pillar face of the IHC (Liberman, 1982; Ohn et al., 2016), high SR pairs reported in this thesis were patched exclusively from the pillar side (chapter 2). Future experiments including a post-patch-clamp reconstruction of the IHC and the recorded bouton will offer a better understanding of the SR gradient along the modiolar/pillar IHC axis. Similar to previous studies (Chapochnikov et al., 2014; Grant et al., 2010), spontaneous EPSCs had variable amplitudes and waveforms, despite their comparable charge. Interestingly, amplitude and percentage of monophasic EPSCs, but not charge, were inversely correlated to SR. This suggests that, on average, synaptic vesicles proceed more rapidly to full-collapse fusion in high SR synapses than at low SR synapse, where fusion pore flickering might be more prevalent and cause a higher fraction of multiphasic EPSCs. The implications of this finding on the subsequent ANF spike generation and sound encoding will be the work of future studies.

\section{Latency}

Sound stimulation evokes an increase in the firing frequency of ANFs in vivo. The latency between sound onset and the first spike recorded (first spike latency) ranges between 2 and 4 ms in absence of phase-locking (Buran et al., 2010; Heil and Neubauer, 2001; Huet et al., 2016; Taberner and Liberman, 2005). This latency is a composite of the acoustic delay of $\sim 0.1 \mathrm{~ms}$, the synaptic delay and the time for spike propagation $(0.15$ to $0.4 \mathrm{~ms})$ to the recording position (Palmer and Russell, 1986). Synaptic delay comprises the openening of $\mathrm{Ca}^{2+}$ channels, fusion of vesicles with the plasma membrane, neurotransmitter diffusion in the synaptic cleft, and binding and opening of the AMPA receptors. A synaptic delay of 0.8 ms was determined in vivo by direct recording of the IHC receptor potential and, in close sucession, of the postsynaptic potential of an adjacent fiber in response to tone bursts at 75 dB SPL (Palmer and Russell, 1986). Paired recordings from IHCs and their contacting boutons have reported synaptic delays -measured from the beginning of the step depolarization to the onset of the EPSC- of $1.27 \pm 0.09 \mathrm{~ms}$ (mouse IHC at physiological 
temperature in this thesis; chapter 2) and of $\sim 1.5 \mathrm{~ms}$ (rat IHC at room temperature; Goutman, 2012; Goutman and Glowatzki, 2011).

Synaptic delays in evoked EPSCs decreased with increasing voltage step intensity (chapter 2; Goutman, 2012), in line with the reduction of the first spike latency with sound intensity observed from ANFs in vivo (Heil and Irvine, 1997; Heil and Neubauer, 2001; Huet et al., 2016). Modelling and experimental observations (Buran et al., 2010; Goutman, 2012; Wittig and Parsons, 2008) have showen that the initial EPSC rates also depend on stimulus intensity, possibly via the recruitment of more $\mathrm{Ca}^{2+}$ channel-vesicle units with increasing depolarization [for review see (Moser et al., 2006b)]. Therefore, the increased accuracy and magnitude of the postsynaptic response with increasing presynaptic $\mathrm{Ca}^{2+}$ influx reflects the close relation between $\mathrm{Ca}^{2+}$ and exocytosis, as reported in ribbon (Beutner et al., 2001; Heidelberger et al., 1994) and conventional synapses (Felmy et al., 2003; Neher and Sakaba, 2008). Furthermore, larger and faster EPSCs elicited with stronger stimuli might promote shorter first spike latencies by speeding up action potential generation in SGN (Rutherford et al., 2012). These observations highlight the presynaptic relevance and contribution to transmission latencies.

The IHC status drastically influences synaptic delay, most likely related to the stimulus history and consequently, to the presynaptic $\mathrm{Ca}^{2+}$ influx (Cho and von Gersdorff, 2014; Goutman, 2012; Goutman and Glowatzki, 2011) and to the RRP (Buran et al., 2010; Wittig and Parsons, 2008). Other strategies to achieve a precise timing include a short distance between the $\mathrm{Ca}^{2+}$ sensor and the $\mathrm{Ca}^{2+}$ channel, high single $\mathrm{Ca}^{2+}$ channel conductance, large numbers of $\mathrm{Ca}^{2+}$ channels, and/or increased open probability of the channel (Wittig and Parsons, 2008). The latter three may cause partial occupation of the $\mathrm{Ca}^{2+}$ binding sites of the sensor, favoring intermediate states before fusion that increase the release probability of such vesicles and thus decrease the latency of release. The relatively depolarized resting potential of IHCs (around -58 mV, Johnson, 2015), in combination with the negative activation voltages of $\mathrm{Ca}_{\mathrm{v}} 1.3$ channels (-65 to $-45 \mathrm{mV}$, (Koschak et al., 2001; Platzer et al., 2000; Xu and Lipscombe, 2001)), are some of the mechanisms employed by IHCs to potentially favor intermediate fusion states. A near-linear $\mathrm{Ca}^{2+}$ dependence of release reported in this thesis (chapter 2) and in previous studies (Brandt et al., 2005; Goutman and Glowatzki, 2007; Johnson et al., 2005; Wong et al., 2014), indicate a $\mathrm{Ca}^{2+}$ nanodomain control of exocytosis as an additional mechanism used by IHCs to achieve high temporal precision of synaptic transmission (further discussed in section 5.3). 
Both experimental (chapter 3 and (Becker et al., 2018; Buran et al., 2010; Frank et al., 2010; Jean et al., 2018)) and computational (Buran et al., 2010; Wittig and Parsons, 2008) studies support a predominant role of the ribbon to ensure a short and reproducible first-exocytosis latency by maintaining a sufficiently large RRP (but for an opposing view, please refer to Lv et al., 2016; Sheets et al., 2017). In mutants lacking the scaffolding protein bassoon, the disruption of the ribbon anchorage was accompanied by a smaller RRP and fewer $\mathrm{Ca}^{2+}$ channels, which resulted in delayed and jittered first sound evoked spikes and reduced compound action potential of the auditory nerve (Buran et al., 2010; Frank et al., 2010; Jing et al., 2013; Khimich et al., 2005). In RIBEYE KO (chapter 3), the resulting ribbonless synapses also showed impaired temporal precision which could be related to the the depolarized shift in the voltage activation of the $\mathrm{Ca}^{2+}$ channels and to a smaller standing RRP consequence of the slower synaptic vesicle replenishment. However, these effects were less pronounced than in bassoon KOs, presumably originated from the partial compensation of the RRP size by a multi active zone organization at RIBEYE KO synapse, such as it is observed in the Calyx of Held (von Gersdorff and Borst, 2002). Additional effects of the absence of bassoon itself can not be excluded. Yet, it remains clear that the particular organization encountered at IHC synapses is crucial for a precise and fast response to stimuli.

\section{Synaptic depression, spike rate adaptation and vesicle replenishment}

During prolonged sound stimulation, adaptation of the onset spike rate of ANFs (Kiang et al., 1965; Westerman and Smith, 1984) is not accompanied by a reduction in the cochlear potential (Davis et al., 1935) and on the IHC receptor potentials (Russell and Sellick, 1978). This suggests mechanisms of adaptation localized at the synapse level (Moser and Beutner, 2000), including inactivation of the $\mathrm{Ca}^{2+}$ current (Johnson and Marcotti, 2008; Moser and Beutner, 2000), synaptic depression [exhaustation of RRP, (Furukawa and Matsuura, 1978; Furukawa et al., 1982; Moser and Beutner, 2000)] and/or desensitization of the postsynaptic glutamate receptors (Goutman, 2017; Goutman and Glowatzki, 2007).

Synaptic depression observed during capacitance measurements most likely reflects RRP depletion with a time course $(\tau)$ around $10 \mathrm{~ms}$ (Moser and Beutner, 2000). Synaptic depression is also evident from paired IHC-bouton recordings (chapter 2 and Goutman, 2017; Goutman and Glowatzki, 2007), where the postsynaptic response decays after the initial peak. The time course of depletion from these recordings can be determined either by fitting an exponential to the average EPSC response ( $\tau=\sim 8 \mathrm{~ms}$, Goutman, 2017; Goutman and Glowatzki, 2007), or by fitting the sum of a single exponential and a line function to the 
EPSC charge ( $\tau=6.47 \mathrm{~ms}$; chapter 2 ). The mild $\mathrm{Ca}^{2+}$ current inactivation (e.g. less than $20 \%$ during $1 \mathrm{~s}$ long depolarizations) does not explain the strong reduction of exocytosis $(>90 \%$; (Goutman and Glowatzki, 2007; Moser and Beutner, 2000)). Synaptic depression determined with capacitance and paired recordings follows a similar time course to the ANFs spike rate adaptation in vivo (rapid adaptation between 1-25 ms, Buran et al., 2010; Spassova et al., 2004; Westerman and Smith, 1984; Yates et al., 1985). The extent of the contribution of AMPA receptor desensitization to adaptation (Goutman, 2017) is still debatable given the potential presynaptic effects of desensitization blockers (Diamond and Jahr, 1995; Dittman and Regehr, 1998).

The degree of adaptation relates to the SR of ANFs in vivo (Taberner and Liberman, 2005; Westerman and Smith, 1984). Modelling of frog hair cell afferent synapses proposed that the differences in adaptation between fibers might result from the IHC's replenishment as well as from differences in fiber's endogenous buffers (Wittig and Parsons, 2008). Supporting the former point, high SR synapses obtained during paired recording experiments (chapter 2) tended to have stronger adaptation of the evoked release rate, as well as faster half time recovery of RRP depletion. Supporting the second point, recent studies reported gradients in the expression of mobile $\mathrm{Ca}^{2+}$ buffers among SGNs (Petitpré et al., 2018; Shrestha et al., 2018).

Despite the initial synaptic depression, IHCs employ effective mechanisms to maintain sustained release during continuous stimulation. It is thought that the ribbon-associated pool of vesicles is released during sustained exocytosis (Guillet et al., 2016; Schnee et al., 2005). Morphological studies, particularly electron tomograms, show about 30-60 vesicles associated to the synaptic ribbon (Chakrabarti and Wichmann, 2019; Chakrabarti et al., 2018). Yet, the high sustained exocytic rates (between 100 and 700 vesicles/s, chapter 2 and (Goutman and Glowatzki, 2007; Johnson et al., 2005; Moser and Beutner, 2000; Pangrsic et al., 2010)) and the indefatigable release of neurotransmitter suggests that additional vesicles must be recruited to the release sites. Replenishment of synaptic vesicles to the ribbon and/or the active zone membrane is scarcely understood. In IHCs, the process appears to involve $\mathrm{Ca}^{2+}$ (Beutner et al., 2001; Schnee et al., 2005; Spassova et al., 2004) and the proteins otoferlin (Michalski et al., 2017; Pangrsic et al., 2010; Strenzke et al., 2016; Vogl et al., 2015, 2016), bassoon (Frank et al., 2010), RIM2 (Jung et al., 2015) and RIM-BP2 (Krinner et al., 2017). 
Results from this thesis (chapter 3) supported the crucial role of the ribbon in replenishment, in line with previous studies (Frank et al., 2010). Yet, the multiple ribbonless 'conventional' active zones found at the synapses of RIBEYE KO might have led to underestimate the replenishment defect. Consequently, studies with inducible RIBEYE KO will be of great importance to rule out developmental compensation mechanisms. Sustained release and number of ribbon-associated synaptic vesicles were also reduced in endophilin mutants (chapter 4), probably reflecting the slowed reformation of synaptic vesicles. Less likely, this phenotype was caused by an impaired otoferlin-mediated replenishment produced by the slightly decreased otoferlin levels in the mutants. The rapid replenishment of the RRP in IHCs might not only support sustained exocytosis (Griesinger et al., 2005; Wittig and Parsons, 2008) but also preserve the temporal precision of the synapse during adaptation. The vesicles retained by the ribbon are mobilized to the active zone during refilling, thus maintaining a large pool for short and reproducible exocytosis during stimulus onset (Buran et al., 2010; Frank et al., 2009, 2010; Wittig and Parsons, 2008).

\section{Membrane recycling and SV reformation}

Given these high rates of transmission, it is logical to think the IHC is equipped with efficient mechanisms for membrane recycling and synaptic vesicle reformation. As a matter of fact, IHCs display three kinetically distinct modes of membrane retrieval - rapid (300 ms), fast (4 s) and slow (20 s half time recovery) - that might resemble the modes of "kiss-and-run" (Beutner et al., 2001; Neef et al., 2014), bulk endocytosis (Jung et al., 2015; Kamin et al., 2014; Neef et al., 2014; Revelo et al., 2014) and clathrin-mediated endocytosis (CME) (Duncker et al., 2013; Jung et al., 2015; Neef et al., 2014). Clathrin (Neef et al., 2014), dynamin 1 (Neef et al., 2014), and synaptojanin-1 (Trapani et al., 2009) appear to mediate slow endocytosis (CME) in IHCs. The adaptor protein AP2, on the other hand, was dispensable for endocytosis in IHCs (Jung et al., 2015), despite being associated to CME in conventional synapses (for review Kaksonen and Roux, 2018; Kononenko et al., 2014). Nonetheless, absence of the subunit AP2 $\mu$ led to accumulation of endosome-like vacuoles and reduced coated structures near the active zone (Jung et al., 2015). These two findings supported the hypothesis that IHCs synaptic vesicles are reformed from bulk-retrieved membrane using clathrin-dependent, as well as clathrin-independent, mechanisms. Bulk retrieval additionally offers more membrane surface for AP-2/clathrin synaptic vesicle reformation. 
It is clear that the mechanisms and players mediating endocytosis at IHC synapses are poorly understood. This is partly due to the lethal phenotype of mice carrying mutations in classical endocytic actors [e.g. endophilin triple KO (Milosevic et al., 2011), synaptojanin KO (Cremona et al., 1999)]. Among them is the family of endophilin A proteins (comprising three isoforms, A1-A3, for review Kaksonen and Roux, 2018). In the present thesis, collaborators and I assessed the effects of the absence of endophilin in exocytosis and endocytosis of IHCs (chapter 4). In line with previous reports (Kjaerulff et al., 2011; Milosevic et al., 2011; Reutens and Begley, 2002), endophilins appeared to be involved in fission and uncoating of synaptic vesicles during CME. This was inferred from the modest accumulation of clathrin coated pits near the active zone and from the prominent accumulation of other clathrin coated structures. This probably underlies the reduced rate of CME endocytosis in endophilin-deficient IHC assessed with capacitance measurements. Accumulation of endosome-like vacuoles with coated-pits upon stimulation further supported the role of endophilins in vesicle reformation and uncoating. Due to the perinatal lethality of the triple knockout, we relied on combinatory KOs of the different isoforms, hence the interpretation was often challenging. Future studies using inducible KO will help to further discern the roles of each endophilin isoform.

\section{Number and organization of $\mathrm{Ca}^{2+}$ channels}

Synaptic transmission is coordinated by $\mathrm{Ca}^{2+}$ entering through presynaptic voltage-gated $\mathrm{Ca}^{2+}$ channels (Llinás et al., 1981a, 1981b). The coupling between $\mathrm{Ca}^{2+}$ channels and the $\mathrm{Ca}^{2+}$ sensor for vesicular release governs synaptic transmission (for review Eggermann et al., 2011). Results from this thesis validated the $\mathrm{Ca}^{2+}$ nanodomain-like control of synaptic transmission in IHCs under physiological conditions. Nanodomain control might ensure that random independent gating of individual $\mathrm{Ca}^{2+}$ channels trigger the fusion of a nearby vesicle (Neher, 1998). This factor, in combination with the hyperpolarized resting membrane potential of the IHC, probably shapes the spontaneous activity and sensitivity of the IHC ribbon synapse.

At IHC active zones, $\mathrm{Ca}^{2+}$ channels are present in stripe-like clusters located beneath the synaptic ribbon (Frank et al., 2010; Neef et al., 2018; Wong et al., 2014). These clusters appear to have similar density of channels, yet variable length (100-600 nm, Neef et al., $2014,2018)$. Hence, the number of $\mathrm{Ca}^{2+}$ channels varies greatly among active zones within one IHC, ranging between 20-330 channels (Neef et al., 2018). Moreover, the voltage 
dependence of $\mathrm{Ca}^{2+}$ influx diverges between different clusters (Frank et al., 2009; Ohn et al., 2016). These two key determinants of $\mathrm{Ca}^{2+}$ influx have opposing spatial gradients along the modiolar/pillar IHC axis. Active zones of the modiolar face have larger $\mathrm{Ca}^{2+}$ channels clusters and greater maximal synaptic $\mathrm{Ca}^{2+}$ influx, but their channels activate at higher potentials (Moser et al., 2019; Ohn et al., 2016; Pangrsic et al., 2018). Which mechanisms determine the number and biophysical properties of $\mathrm{Ca}^{2+}$ channels in IHC ribbon synapses?

The expression of $\mathrm{Ca}^{2+}$ channel subunits (Frank et al., 2009; Kuhn et al., 2009), splice variants (Bock et al., 2011; Shen et al., 2006; Vincent et al., 2017), and interacting partners [GIPC3, (Ohn et al., 2016); bassoon (Frank et al., 2010; Jing et al., 2013); RIM (Picher et al., 2017b); CaBP2 (Picher et al., 2017a); RIM-BP (Krinner et al., 2017)] can affect both the number of $\mathrm{Ca}^{2+}$ channels as well as modulate their functional properties. In IHCs, the core $\mathrm{Ca}^{2+}$ selective channels pore is likely formed by the subunits $\mathrm{Ca}_{\mathrm{v}} 1.3 \alpha, \mathrm{Ca}_{\mathrm{v}} \beta 2$ (Neef et al., 2009), and $\mathrm{Ca}_{\mathrm{v}} \alpha_{2} \delta$ (Fell et al., 2016). The $\mathrm{Ca}_{\mathrm{v}} \beta 2$ subunit promotes the trafficking of the $\mathrm{Ca}^{2+}$ channel and its stabilization at the plasma membrane and thus, regulates the abundance of $\mathrm{Ca}_{\mathrm{v}} 1.3$ channels at the active zone (Neef et al., 2009). The $\alpha_{2} \delta_{2}$ subunit appears to reduce the turnover of the channel and exerts a role in the channel gating (Fell et al., 2016). Its deletion results in reduced $\mathrm{Ca}^{2+}$ current and a misalignment of the $\mathrm{Ca}^{2+}$ cluster to the AMPA receptors (Fell et al., 2016). Alternative splicing in the $\mathrm{COOH}$ terminus of the $\mathrm{Ca}_{\mathrm{v}} 1.3 \alpha$ subunit generates short and long variants with different biophysical properties (Singh et al., 2008; Tan et al., 2011). The properties will depend on the presence or absence of the proximal and distal COOH-terminal regulatory domain (Bock et al., 2011; Tan et al., 2011). This domain reduces channel open probability and shifts the voltage dependence to more positive potentials. It also weakens calmodulin mediated $\mathrm{Ca}^{2+}$ dependent inactivation (Scharinger et al., 2015). However, replacing the distal domain with a hemagglutinin tag did not change the voltage dependence of $\mathrm{Ca}^{2+}$ influx in IHC from KI mice, in which long $\mathrm{Ca}_{\mathrm{v}} 1.3$ channels should biophysically resemble the short splice isoform. It remains to be determined whether the subunit composition and/or presence of splice variants differs between modiolar and pillar synapses.

Among the interacting partners, RIM2, RIM-BP2 and GIPC3 appear to influence the abundance and properties of $\mathrm{Ca}^{2+}$ channels. RIM2 $\alpha$ and $\beta$ isoforms are present at the base of the ribbon, where they promote the abundance of channels at the active zone (Jung et al., 2015). $\mathrm{Ca}^{2+}$ influx, and consequent exocytosis, is reduced in IHCs lacking these two isoforms, yet there is no impact in the $\mathrm{Ca}^{2+}$ channel cluster shape and organization (Jung et 
al., 2015). RIM-BP2 positively regulates the number of channels (Krinner et al., 2017). GIPC3 is one of the strongest candidates for the heterogeneity in of $\mathrm{Ca}^{2+}$ channel voltage activation. Absence of this cytosolic scaffold protein increases the whole cell IHC $\mathrm{Ca}^{2+}$ current, causes an overall hyperpolarizing shift in the activation of the $\mathrm{Ca}^{2+}$ channels, and, most strikingly, reverses the modiolar/pillar gradient of maximal $\mathrm{Ca}^{2+}$ influx (Ohn et al., 2016). These presynaptic changes are accompanied by increased spontaneous spike rates, sound-onset firing and narrower dynamic ranges in SGNs from mutant mice. These findings support the hypothesis that the diversity in response properties might come from the presynaptic clustering of $\mathrm{Ca}^{2+}$ channels.

In this thesis, collaborators and I found that RIBEYE/ribbon and endophilin also mediate $\mathrm{Ca}^{2+}$ channels properties in mouse IHC. In RIBEYE KO, the abundance of $\mathrm{Ca}^{2+}$ channels and maximal $\mathrm{Ca}^{2+}$ influx remained the same, while some functional properties were distorted. For instance, $\mathrm{Ca}^{2+}$ channels required higher potentials for their activation and inactivation was enhanced. These functional changes were accompanied by reduced exocytosis for weak depolarizations, reduced spontaneous firing rates and a lower temporal precision. A direct interaction between the ribbon and the $\mathrm{Ca}^{2+}$ channels has not been shown and therefore, the exact mechanism of the observed changes remains unknown. EGTA experiments (Becker et al., 2018), superresolution imaging of the active zone and whole cell $\mathrm{Ca}^{2+}$ current measurements suggested unaffected coupling distance and number of $\mathrm{Ca}^{2+}$ channels (chapter 3 and Becker et al., 2018). Contrary, $\mathrm{Ca}^{2+}$ channel cluster size, and wholecell $\mathrm{Ca}^{2+}$ influx and inactivation were reduced in endophilin deficient IHCs, in line with the reported interaction between endophilin $\mathrm{A} 1$ and $\mathrm{Ca}^{2+}$ channels (chapter 4).

\section{Diversity of SGN responses and heterogeneity}

High SR synapses studied with paired recordings showed significantly lower voltage thresholds of release and a trend to shorter latencies, faster recovery from RRP depletion (chapter 2), findings that are compatible with the in vivo phenotype of high SR, low sound threshold ANFs (Bourien et al., 2014; Relkin and Doucet, 1991; Rhode and Smith, 1985; Taberner and Liberman, 2005). Thus, these results support the hypothesis that heterogeneity of active zones within individual IHCs might underlie the diversity of spontaneous and sound-evoked SGN firing properties (Frank et al., 2009; Ohn et al., 2016). Yet, a contribution from the IHC's resting state to the heterogeneity between boutons cannot be ruled out. The differences in the threshold of ANFs could also be accounted by differences 
in IHCs of very similar CFs. The present thesis (chapter 2) shows that high SR synapses also contacted IHCs with a more hyperpolarized activation potential of whole cell $\mathrm{Ca}^{2+}$ influx. However, recordings from several boutons contacting one IHC were not systematically achieved, thus future experiments with optical readouts of $\mathrm{Ca}^{2+}$ influx and/or exocytosis might give insights about the heterogeneity among IHCs and synapses. Estimates of RRP size and vesicular release probability (time constant of RRP release), congruent with literature, were comparable among synapses and thus, suggest that this might not be a contributing factor to the diversity. This finding is surprising as the heterogeneity of AZ size and $\mathrm{Ca}^{2+}$ channel cluster (e.g. Frank et al., 2009; Neef et al., 2018; Ohn et al., 2016; Wong et al., 2014) would lead one to assume that the RRP size should vary substantially. However, the relatively small sample size of synapses recorded from the modiolar and pillar side, together with the mild gradient of size and maximal $\mathrm{Ca}^{2+}$ influx of IHC AZs ("salt and pepper" distribution), might have precluded the present thesis to reveal such differences.

Dynamic range and voltage sensitivity of release were indistinguishable between low and high SR synapses. This contrasts the characteristic shapes of the rate level functions recorded in vivo from low SR ANFs, with shallow rise with late saturation, and high SR ANFs, with steep rise with fast saturation. The findings from this thesis (chapter 2) support the idea that the non-saturated rate level function from the low SR fibers might arrise from the non-linear saturating properties of the basilar membrane (Sachs and Abbas, 1974; Sachs et al., 1989; Yates et al., 1990, discussed in Ohlemiller et al, 1991). Alternatively, the shape of the relationship of firing rate and sound pressure level could be determined downstream of glutamate release and AMPA receptor activation. Additionally, differences in the number and/or properties of the glutamate receptors unlikely produce the heterogeneous response, contrary to previously proposed (Grant et al., 2010). Postsynaptic excitability differences appear more probable to contribute to the distinct rate level functions recorded in vivo. In fact, high SR fibers have thicker diameters and are highly enriched in mitochondria (Liberman, 1982; Merchan-Perez and Liberman, 1996; Shrestha et al., 2018; Sun et al., 2018), two characteristics that potentially mediate and reflect faster conduction velocities and higher activity levels. Moreover, other components, including voltage-gated channels and mobile buffers, show gradients in their expression levels among subtypes of SGNs (Shrestha et al., 2018; Sun et al., 2018). Future studies combining paired patch clamp recordings, fiber tracing and immunostainings of molecular markers will be crucial to find morphological correlates to the response properties of individual IHC synapses. 


\section{List of abbreviations}

$\begin{array}{ll}\text { ABR } & \text { Auditory brainstem response } \\ \text { AC } & \text { Alternating current } \\ \text { AMPA } & \alpha \text {-Amino-3-hydroxy-5-methyl-4-isoxazolepropionic acid receptor } \\ \text { ANF } & \text { Auditory Nerve Fiber } \\ \text { AP } & \text { Action potential } \\ \text { AZ } & \text { Active zone }\end{array}$

BAPTA 1,2-bis(o-aminophenoxy)ethane- $\mathrm{N}, \mathrm{N}, \mathrm{N}^{\prime}, \mathrm{N}^{\prime}$-tetraacetic acid, "fast" $\mathrm{Ca}^{2+}$ chelator

$\left[\mathrm{Ca}^{2+}\right] \quad \mathrm{Ca}^{2+}$ concentration

$\mathbf{C}_{2} \quad \mathrm{Ca}^{2+}$-binding protein domain

CaBP $\quad \mathrm{Ca}^{2+}$ binding protein

CaM Calmodulin

Cav1.3 Voltage-gated $\mathrm{Ca}^{2+}$ channel, L-type, pore-forming $\alpha$-1D subunit

Cavß Auxiliary $\beta$-subunit of voltage-gated $\mathrm{Ca}^{2+}$ channel

Cava2 $\quad$ Auxiliary $\alpha 2 \delta$-subunit of voltage-gated $\mathrm{Ca}^{2+}$ channel

$\Delta \mathbf{C}_{\mathbf{m}} \quad$ Membrane capacitance changes

CtBP2 C-terminal binding protein 2

DC Direct current

DPOAE Distortion product otoacoustic emissions

EGTA Ethylene glycol-bis(2-aminoethylether)-N,N,N',N'-tetraacetic acid, "slow" $\mathrm{Ca}^{2+}$ chelator

EPSC Excitatory postsynaptic current

EPSP Excitatory postsynaptic potential

GluA Glutamate receptor subunit type, comprising AMPA receptors

HCN Hyperpolarization-activated, cyclic nucleotide-gated nonspecificationic current $\left(\mathrm{I}_{\mathrm{h}}\right)$

IHC Inner hair cell

ISI Interstimulus interval

IV Current-voltage relationship

k Slope

KO Knockout

KV Voltage-gated $\mathrm{K}^{+}$channel 


\begin{tabular}{|c|c|}
\hline LJP & Liquid junction potential \\
\hline$m$ & $\begin{array}{l}\text { Apparent } \mathrm{Ca}^{2+} \text { cooperativity of exocytosis; from a power function fit to the } \\
\text { relationship between exocytosis and } \mathrm{Ca}^{2+} \text { influx }\end{array}$ \\
\hline MET & Mechanoelectrical transduction \\
\hline MVR & Multivesicular release \\
\hline Nav & Voltage-gated $\mathrm{Na}^{+}$channel \\
\hline $\mathbf{N}_{\text {slots }}$ & Number of release sites \\
\hline OHC & Outer hair cell \\
\hline PSD & Postsynaptic density \\
\hline $\mathbf{p X}$ & Postnatal day $X$ \\
\hline $\mathbf{Q}_{\mathbf{C a}}$ & Calcium charge \\
\hline $\mathbf{Q}_{\text {EPSC }}$ & EPSC charge \\
\hline RIM & Rab3-interacting molecule \\
\hline RIM-BP & RIM binding protein \\
\hline RRP & Readily releasable pool of vesicles \\
\hline s.d. & Standard deviation \\
\hline s.e.m. & Standard error of the mean \\
\hline SGN & $\begin{array}{l}\text { Type I spiral ganglion neuron, also called auditory nerve fiber, cochlear nerve } \\
\text { fiber, or auditory nerve single-unit }\end{array}$ \\
\hline SNARE & $\begin{array}{l}\text { Soluble NSF attachment protein receptors, including SNAP, syntaxin, and } \\
\text { synaptobrevin proteins }\end{array}$ \\
\hline SPL & Sound pressure level \\
\hline SR & Spontaneous rate of EPSCs or APs of a SGN \\
\hline STED & Stimulated emission depletion microscopy \\
\hline SV & Synaptic vesicle \\
\hline UVR & Univesicular release \\
\hline $\mathbf{V}_{\text {glut }}$ & Vesicular glutamate transporter \\
\hline $\mathbf{V}_{\mathbf{h}}$ & Voltage for half activation \\
\hline
\end{tabular}




\section{References}

Adler, E.M., Augustine, G.J., Duffy, S.N., and Charlton, M.P. (1991). Alien intracellular calcium chelators attenuate neurotransmitter release at the squid giant synapse. J. Neurosci. $11,1496-1507$.

Ashmore, J. (2008). Cochlear Outer Hair Cell Motility. Physiol. Rev. 88, 173-210.

Ashmore, J.F. (1987). A fast motile response in guinea-pig outer hair cells: the cellular basis of the cochlear amplifier. J. Physiol. 388, 323-347.

Assad, J.A., Shepherd, G.M.G., and Corey, D.P. (1991). Tip-link integrity and mechanical transduction in vertebrate hair cells. Neuron 7, 985-994.

Augustine, G.J., Adler, E.M., and Charlton, M.P. (1991). The calcium signal for transmitter secretion from presynaptic nerve terminals. Ann. N. Y. Acad. Sci. 635, 365-381.

Barbary, A.E. (1991). Auditory nerve of the normal and jaundiced rat. I. Spontaneous discharge rate and cochlear nerve histology. Hear. Res. 54, 75-90.

Becker, L., Schnee, M.E., Niwa, M., Sun, W., Maxeiner, S., Talaei, S., Kachar, B., Rutherford, M.A., and Ricci, A.J. (2018). The presynaptic ribbon maintains vesicle populations at the hair cell afferent fiber synapse. ELife 7, e30241.

Ben Johny, M., Yang, P.S., Bazzazi, H., and Yue, D.T. (2013). Dynamic switching of calmodulin interactions underlies $\mathrm{Ca} 2+$ regulation of CaV1.3 channels. Nat. Commun. 4, 1717.

Berglund, A.M., and Ryugo, D.K. (1987). Hair cell innervation by spiral ganglion neurons in the mouse. J. Comp. Neurol. 255, 560-570.

Berjukow, S., Marksteiner, R., Gapp, F., Sinnegger, M.J., and Hering, S. (2000). Molecular Mechanism of Calcium Channel Block by Isradipine: ROLE OF A DRUG-INDUCED INACTIVATED CHANNEL CONFORMATION. J. Biol. Chem. 275, 22114-22120.

Beurg, M., Fettiplace, R., Nam, J.-H., and Ricci, A.J. (2009). Localization of inner hair cell mechanotransducer channels using high-speed calcium imaging. Nat. Neurosci. 12, 553558.

Beurg, M., Michalski, N., Safieddine, S., Bouleau, Y., Schneggenburger, R., Chapman, E.R., Petit, C., and Dulon, D. (2010). Control of exocytosis by synaptotagmins and otoferlin in auditory hair cells. J. Neurosci. Off. J. Soc. Neurosci. 30, 13281-13290.

Beutner, D., and Moser, T. (2001). The Presynaptic Function of Mouse Cochlear Inner Hair Cells during Development of Hearing. J. Neurosci. 21, 4593-4599.

Beutner, D., Voets, T., Neher, E., and Moser, T. (2001). Calcium Dependence of Exocytosis and Endocytosis at the Cochlear Inner Hair Cell Afferent Synapse. Neuron 29, 681-690. 
Bock, G., Gebhart, M., Scharinger, A., Jangsangthong, W., Busquet, P., Poggiani, C., Sartori, S., Mangoni, M.E., Sinnegger-Brauns, M.J., Herzig, S., et al. (2011). Functional Properties of a Newly Identified C-terminal Splice Variant of Cav1.3 L-type Ca2+ Channels. J. Biol. Chem. 286, 42736-42748.

Bourien, J., Tang, Y., Batrel, C., Huet, A., Lenoir, M., Ladrech, S., Desmadryl, G., Nouvian, R., Puel, J.-L., and Wang, J. (2014). Contribution of auditory nerve fibers to compound action potential of the auditory nerve. J. Neurophysiol. 112, 1025-1039.

Brandt, A., Striessnig, J., and Moser, T. (2003). CaV1. 3 channels are essential for development and presynaptic activity of cochlear inner hair cells. J. Neurosci. 23, 1083210840.

Brandt, A., Khimich, D., and Moser, T. (2005). Few CaV1. 3 channels regulate the exocytosis of a synaptic vesicle at the hair cell ribbon synapse. J. Neurosci. 25, 11577.

Braude, J.P., Vijayakumar, S., Baumgarner, K., Laurine, R., Jones, T.A., Jones, S.M., and Pyott, S.J. (2015). Deletion of Shank1 has minimal effects on the molecular composition and function of glutamatergic afferent postsynapses in the mouse inner ear. Hear. Res. 321, 5264.

Buran, B.N., Strenzke, N., Neef, A., Gundelfinger, E.D., Moser, T., and Liberman, M.C. (2010). Onset coding is degraded in auditory nerve fibers from mutant mice lacking synaptic ribbons. J. Neurosci. Off. J. Soc. Neurosci. 30, 7587-7597.

Chakrabarti, R., and Wichmann, C. (2019). Nanomachinery Organizing Release at Neuronal and Ribbon Synapses. Int. J. Mol. Sci. 20, 2147.

Chakrabarti, R., Michanski, S., and Wichmann, C. (2018). Vesicle sub-pool organization at inner hair cell ribbon synapses. EMBO Rep. e44937.

Chapochnikov, N.M., Takago, H., Huang, C.-H., Pangršič, T., Khimich, D., Neef, J., Auge, E., Göttfert, F., Hell, S.W., Wichmann, C., et al. (2014). Uniquantal Release through a Dynamic Fusion Pore Is a Candidate Mechanism of Hair Cell Exocytosis. Neuron 17, 13891403.

Cheatham, M.A. (2004). Cochlear function in Prestin knockout mice. J. Physiol. 560, 821830.

Chen, X.H., Bezprozvanny, I., and Tsien, R.W. (1996). Molecular basis of proton block of L-type Ca2+ channels. J. Gen. Physiol. 108, 363-374.

Cho, S., and von Gersdorff, H. (2012). Ca2+ influx and neurotransmitter release at ribbon synapses. Cell Calcium 52, 208-216.

Cho, S., and von Gersdorff, H. (2014). Proton-Mediated Block of Ca2+ Channels during Multivesicular Release Regulates Short-Term Plasticity at an Auditory Hair Cell Synapse. J. Neurosci. 34, 15877-15887.

Christel, C.J., Schaer, R., Wang, S., Henzi, T., Kreiner, L., Grabs, D., Schwaller, B., and Lee, A. (2012). Calretinin Regulates Ca2+-dependent Inactivation and Facilitation of Cav2.1 Ca2+ Channels through a Direct Interaction with the $\alpha 12.1$ Subunit. J. Biol. Chem. 287, 39766-39775. 
Corey, D.P., and Hudspeth, A.J. (1979). Ionic basis of the receptor potential in a vertebrate hair cell. Nature 281, 675-677.

Cremona, O., Di Paolo, G., Wenk, M.R., Lüthi, A., Kim, W.T., Takei, K., Daniell, L., Nemoto, Y., Shears, S.B., Flavell, R.A., et al. (1999). Essential role of phosphoinositide metabolism in synaptic vesicle recycling. Cell 99, 179-188.

Cui, G., Meyer, A.C., Calin-Jageman, I., Neef, J., Haeseleer, F., Moser, T., and Lee, A. (2007). Ca2+-binding proteins tune Ca2+-feedback to Cav1. 3 channels in mouse auditory hair cells. J. Physiol. 585, 791-803.

Davies, C., Tingley, D., Kachar, B., Wenthold, R.J., and Petralia, R.S. (2001). Distribution of members of the PSD-95 family of MAGUK proteins at the synaptic region of inner and outer hair cells of the guinea pig cochlea. Synapse 40, 258-268.

Davis, H., Derbyshire, A.J., Kemp, E.H., Lurie, M.H., and Upton, M. (1935). EXPERIMENTAL STIMULATION DEAFNESS. Science 81, 101-102.

DeVries, S.H. (2001). Exocytosed Protons Feedback to Suppress the Ca2+ Current in Mammalian Cone Photoreceptors. Neuron 32, 1107-1117.

Diamond, J.S., and Jahr, C.E. (1995). Asynchronous release of synaptic vesicles determines the time course of the AMPA receptor-mediated EPSC. Neuron 15, 1097-1107.

Dick, O., tom Dieck, S., Altrock, W.D., Ammermüller, J., Weiler, R., Garner, C.C., Gundelfinger, E.D., and Brandstätter, J.H. (2003). The Presynaptic Active Zone Protein Bassoon Is Essential for Photoreceptor Ribbon Synapse Formation in the Retina. Neuron 37, $775-786$.

Dittman, J.S., and Regehr, W.G. (1998). Calcium Dependence and Recovery Kinetics of Presynaptic Depression at the Climbing Fiber to Purkinje Cell Synapse. J. Neurosci. 18, 6147-6162.

Dou, H., Vazquez, A.E., Namkung, Y., Chu, H., Cardell, E.L., Nie, L., Parson, S., Shin, H.S., and Yamoah, E.N. (2004). Null mutation of alpha1D Ca2+ channel gene results in deafness but no vestibular defect in mice. J. Assoc. Res. Otolaryngol. JARO 5, 215-226.

Duncker, S.V., Franz, C., Kuhn, S., Schulte, U., Campanelli, D., Brandt, N., Hirt, B., Fakler, B., Blin, N., Ruth, P., et al. (2013). Otoferlin Couples to Clathrin-Mediated Endocytosis in Mature Cochlear Inner Hair Cells. J. Neurosci. 33, 9508-9519.

Eggermann, E., Bucurenciu, I., Goswami, S.P., and Jonas, P. (2012). Nanodomain coupling between $\mathrm{Ca}^{2+}$ channels and sensors of exocytosis at fast mammalian synapses. Nat. Rev. Neurosci. 13, 7-21.

Evans, E.F. (1972). The frequency response and other properties of single fibres in the guinea-pig cochlear nerve. J. Physiol. 226, 263-287.

Fell, B., Eckrich, S., Blum, K., Eckrich, T., Hecker, D., Obermair, G.J., Münkner, S., Flockerzi, V., Schick, B., and Engel, J. (2016). $\alpha 2 \delta 2$ Controls the Function and TransSynaptic Coupling of Cav1.3 Channels in Mouse Inner Hair Cells and Is Essential for Normal Hearing. J. Neurosci. Off. J. Soc. Neurosci. 36, 11024-11036. 
Felmy, F., Neher, E., and Schneggenburger, R. (2003). Probing the Intracellular Calcium Sensitivity of Transmitter Release during Synaptic Facilitation. Neuron 37, 801-811.

Fettiplace, R. (2017). Hair Cell Transduction, Tuning, and Synaptic Transmission in the Mammalian Cochlea. Compr. Physiol. 7, 1197-1227.

Frank, T., Khimich, D., Neef, A., and Moser, T. (2009). Mechanisms contributing to synaptic $\mathrm{Ca} 2+$ signals and their heterogeneity in hair cells. Proc. Natl. Acad. Sci. 106, 4483-4488.

Frank, T., Rutherford, M.A., Strenzke, N., Neef, A., Pangršič, T., Khimich, D., Fejtova, A., Gundelfinger, E.D., Liberman, M.C., Harke, B., et al. (2010). Bassoon and the synaptic ribbon organize $\mathrm{Ca}^{2}+$ channels and vesicles to add release sites and promote refilling. Neuron 68, 724-738.

Fuchs, P.A., Evans, M.G., and Murrow, B.W. (1990). Calcium currents in hair cells isolated from the cochlea of the chick. J. Physiol. 429, 553-568.

Fuchs, P.A., Glowatzki, E., and Moser, T. (2003). The afferent synapse of cochlear hair cells. Curr. Opin. Neurobiol. 13, 452-458.

Furukawa, T., and Matsuura, S. (1978). Adaptive rundown of excitatory post-synaptic potentials at synapses between hair cells and eight nerve fibres in the goldfish. J. Physiol. 276, 193-209.

Furukawa, T., Kuno, M., and Matsuura, S. (1982). Quantal analysis of a decremental response at hair cell-afferent fibre synapses in the goldfish sacculus. J. Physiol. 322, 181.

von Gersdorff, H., and Borst, J.G.G. (2002). Short-term plasticity at the calyx of held. Nat Rev Neurosci 3, 53-64.

Glowatzki, E., and Fuchs, P.A. (2002). Transmitter release at the hair cell ribbon synapse. Nat. Neurosci. 5, 147-154.

Goutman, J.D. (2012). Transmitter Release from Cochlear Hair Cells Is Phase Locked to Cyclic Stimuli of Different Intensities and Frequencies. J. Neurosci. 32, 17025-17036.

Goutman, J.D. (2017). Mechanisms of synaptic depression at the hair cell ribbon synapse that support auditory nerve function. Proc. Natl. Acad. Sci. U. S. A.

Goutman, J.D., and Glowatzki, E. (2007). Time course and calcium dependence of transmitter release at a single ribbon synapse. Proc. Natl. Acad. Sci. 104, 16341-16346.

Goutman, J.D., and Glowatzki, E. (2011). Short-Term Facilitation Modulates Size and Timing of the Synaptic Response at the Inner Hair Cell Ribbon Synapse. J. Neurosci. 31, 7974-7981.

Grabner, C.P., and Moser, T. (2018). Individual synaptic vesicles mediate stimulated exocytosis from cochlear inner hair cells. Proc. Natl. Acad. Sci. 115, 12811-12816.

Grant, L., Yi, E., and Glowatzki, E. (2010). Two Modes of Release Shape the Postsynaptic Response at the Inner Hair Cell Ribbon Synapse. J. Neurosci. 30, 4210-4220. 
Gratz, S.J., Goel, P., Bruckner, J.J., Hernandez, R.X., Khateeb, K., Macleod, G.T., Dickman, D., and O'Connor-Giles, K.M. (2019). Endogenous Tagging Reveals Differential Regulation of $\mathrm{Ca} 2+$ Channels at Single Active Zones during Presynaptic Homeostatic Potentiation and Depression. J. Neurosci. Off. J. Soc. Neurosci. 39, 2416-2429.

Graydon, C.W., Cho, S., Li, G.-L., Kachar, B., and von Gersdorff, H. (2011). Sharp Ca2+ Nanodomains beneath the Ribbon Promote Highly Synchronous Multivesicular Release at Hair Cell Synapses. J. Neurosci. 31, 16637-16650.

Griesinger, C.B., Richards, C.D., and Ashmore, J.F. (2002). FM1-43 reveals membrane recycling in adult inner hair cells of the mammalian cochlea. J. Neurosci. 22, 3939.

Griesinger, C.B., Richards, C.D., and Ashmore, J.F. (2005). Fast vesicle replenishment allows indefatigable signalling at the first auditory synapse. Nature 435, 212-215.

Guillet, M., Sendin, G., Bourien, J., Puel, J.-L., and Nouvian, R. (2016). Actin Filaments Regulate Exocytosis at the Hair Cell Ribbon Synapse. J. Neurosci. 36, 649-654.

Hackney, C.M., Mahendrasingam, S., Penn, A., and Fettiplace, R. (2005). The concentrations of calcium buffering proteins in mammalian cochlear hair cells. J. Neurosci. $25,7867$.

Hardie, J., and Lee, A. (2016). Decalmodulation of Cav1 channels by CaBPs. Channels 10, $33-37$.

Harris, D.M., and Dallos, P. (1979). Forward masking of auditory nerve fiber responses. J. Neurophysiol. 42, 1083-1107.

Heidelberger, R., Heinemann, C., Neher, E., and Matthews, G. (1994). Calcium dependence of the rate of exocytosis in a synaptic terminal. Nature $371,513-515$.

Heil, P., and Irvine, D.R. (1997). First-spike timing of auditory-nerve fibers and comparison with auditory cortex. J. Neurophysiol. 78, 2438-2454.

Heil, P., and Neubauer, H. (2001). Temporal integration of sound pressure determines thresholds of auditory-nerve fibers. J. Neurosci. Off. J. Soc. Neurosci. 21, 7404-7415.

Heil, P., and Peterson, A.J. (2015). Basic response properties of auditory nerve fibers: a review. Cell Tissue Res. 361, 129-158.

Hess, P., Lansman, J.B., and Tsien, R.W. (1984). Different modes of Ca channel gating behaviour favoured by dihydropyridine $\mathrm{Ca}$ agonists and antagonists. Nature $311,538-544$.

Hildebrand, J.D., and Soriano, P. (2002). Overlapping and unique roles for C-terminal binding protein 1 (CtBP1) and CtBP2 during mouse development. Mol. Cell. Biol. 22, 52965307.

Holderith, N., Lorincz, A., Katona, G., Rózsa, B., Kulik, A., Watanabe, M., and Nusser, Z. (2012). Release probability of hippocampal glutamatergic terminals scales with the size of the active zone. Nat. Neurosci. 15, 988-997.

Holt, M., Cooke, A., Wu, M.M., and Lagnado, L. (2003). Bulk Membrane Retrieval in the Synaptic Terminal of Retinal Bipolar Cells. J. Neurosci. 23, 1329-1339. 
Hoppa, M.B., Lana, B., Margas, W., Dolphin, A.C., and Ryan, T.A. (2012). $\alpha 2 \delta$ expression sets presynaptic calcium channel abundance and release probability. Nature 486, 122-125.

Howard, J., and Hudspeth, A.J. (1987). Mechanical relaxation of the hair bundle mediates adaptation in mechanoelectrical transduction by the bullfrog's saccular hair cell. Proc. Natl. Acad. Sci. U. S. A. 84, 3064-3068.

Huang, C.-H., and Moser, T. (2018). Ca2+ Regulates the Kinetics of Synaptic Vesicle Fusion at the Afferent Inner Hair Cell Synapse. Front. Cell. Neurosci. 12, 364.

Huet, A., Batrel, C., Tang, Y., Desmadryl, G., Wang, J., Puel, J.-L., and Bourien, J. (2016). Sound coding in the auditory nerve of gerbils. Hear. Res.

Hull, C., Studholme, K., Yazulla, S., and von Gersdorff, H. (2006). Diurnal changes in exocytosis and the number of synaptic ribbons at active zones of an ON-type bipolar cell terminal. J. Neurophysiol. 96, 2025-2033.

Imig, C., Min, S.-W., Krinner, S., Arancillo, M., Rosenmund, C., Südhof, T.C., Rhee, J., Brose, N., and Cooper, B.H. (2014). The morphological and molecular nature of synaptic vesicle priming at presynaptic active zones. Neuron $84,416-431$.

James, D.J., and Martin, T.F.J. (2013). CAPS and Munc13: CATCHRs that SNARE Vesicles. Front. Endocrinol. 4, 187.

Jaramillo, F., and Hudspeth, A.J. (1991). Localization of the hair cell's transduction channels at the hair bundle's top by iontophoretic application of a channel blocker. Neuron 7, 409420 .

Jarsky, T., Tian, M., and Singer, J.H. (2010). Nanodomain control of exocytosis is responsible for the signaling capability of a retinal ribbon synapse. J. Neurosci. Off. J. Soc. Neurosci. 30, 11885-11895.

Jean, P., Morena, D.L. de la, Michanski, S., Tobón, L.M.J., Chakrabarti, R., Picher, M.M., Neef, J., Jung, S., Gültas, M., Maxeiner, S., et al. (2018). The synaptic ribbon is critical for sound encoding at high rates and with temporal precision. ELife 7, e29275.

Jean, P., Özçete, Ö.D., Tarchini, B., and Moser, T. (2019). Intrinsic planar polarity mechanisms influence the position-dependent regulation of synapse properties in inner hair cells. Proc. Natl. Acad. Sci. 116, 9084-9093.

Jing, Z., Rutherford, M.A., Takago, H., Frank, T., Fejtova, A., Khimich, D., Moser, T., and Strenzke, N. (2013). Disruption of the presynaptic cytomatrix protein bassoon degrades ribbon anchorage, multiquantal release, and sound encoding at the hair cell afferent synapse. J. Neurosci. 33, 4456-4467.

Johnson, D.H. (1980). The relationship between spike rate and synchrony in responses of auditory-nerve fibers to single tones. J. Acoust. Soc. Am. 68, 1115-1122.

Johnson, S.L. (2015). Membrane properties specialize mammalian inner hair cells for frequency or intensity encoding. ELife 4, e08177.

Johnson, D.H., and Kiang, N.Y. (1976). Analysis of discharges recorded simultaneously from pairs of auditory nerve fibers. Biophys. J. 16, 719-734. 
Johnson, S.L., and Marcotti, W. (2008). Biophysical properties of CaV1.3 calcium channels in gerbil inner hair cells. J. Physiol. 586, 1029-1042.

Johnson, S.L., Marcotti, W., and Kros, C.J. (2005). Increase in efficiency and reduction in $\mathrm{Ca} 2+$ dependence of exocytosis during development of mouse inner hair cells. J. Physiol. $563,177-191$.

Johnson, S.L., Adelman, J.P., and Marcotti, W. (2007). Genetic deletion of SK2 channels in mouse inner hair cells prevents the developmental linearization in the $\mathrm{Ca} 2+$ dependence of exocytosis. J. Physiol. 583, 631-646.

Johnson, S.L., Forge, A., Knipper, M., Münkner, S., and Marcotti, W. (2008). Tonotopic variation in the calcium dependence of neurotransmitter release and vesicle pool replenishment at mammalian auditory ribbon synapses. J. Neurosci. 28, 7670-7678.

Johnson, S.L., Franz, C., Knipper, M., and Marcotti, W. (2009). Functional maturation of the exocytotic machinery at gerbil hair cell ribbon synapses. J. Physiol. 587, 1715-1726.

Johnson, S.L., Olt, J., Cho, S., von Gersdorff, H., and Marcotti, W. (2017). The Coupling between $\mathrm{Ca} 2+$ Channels and the Exocytotic $\mathrm{Ca} 2+$ Sensor at Hair Cell Ribbon Synapses Varies Tonotopically along the Mature Cochlea. J. Neurosci. 37, 2471-2484.

Jung, S., Maritzen, T., Wichmann, C., Jing, Z., Neef, A., Revelo, N.H., Al-Moyed, H., Meese, S., Wojcik, S.M., Panou, I., et al. (2015). Disruption of adaptor protein $2 \mu$ (AP- $2 \mu$ ) in cochlear hair cells impairs vesicle reloading of synaptic release sites and hearing. EMBO J. 34, 2686-2702.

Kaksonen, M., and Roux, A. (2018). Mechanisms of clathrin-mediated endocytosis. Nat. Rev. Mol. Cell Biol. 19, 313-326.

Kamin, D., Revelo, N.H., and Rizzoli, S.O. (2014). FM Dye Photo-Oxidation as a Tool for Monitoring Membrane Recycling in Inner Hair Cells. PLoS ONE 9, e88353.

Kandel, E.R., Schwartz, J.H., and Jessell, T. (2012). Principles of neural science (New York: McGraw-Hill Medical).

Kantardzhieva, A.V., Liberman, M.C., and Sewell, W.F. (2013). Quantitative analysis of ribbons, vesicles, and cisterns at the cat inner hair cell synapse: correlations with spontaneous rate. J. Comp. Neurol. 521, 3260-3271.

Kavalali, E.T., and Jorgensen, E.M. (2014). Visualizing presynaptic function. Nat. Neurosci. $17,10-16$.

Keen, E.C., and Hudspeth, A.J. (2006). Transfer characteristics of the hair cell's afferent synapse. Proc. Natl. Acad. Sci. 103, 5537-5542.

Kharkovets, T., Dedek, K., Maier, H., Schweizer, M., Khimich, D., Nouvian, R., Vardanyan, V., Leuwer, R., Moser, T., and Jentsch, T.J. (2006). Mice with altered KCNQ4 K+ channels implicate sensory outer hair cells in human progressive deafness. EMBO J. 25, 642-652.

Khimich, D., Nouvian, R., Pujol, R., tom Dieck, S., Egner, A., Gundelfinger, E.D., and Moser, T. (2005). Hair cell synaptic ribbons are essential for synchronous auditory signalling. Nature 434, 889-894. 
Kiang, N.Y., Watanabe, T., Thomas, E.C., and Clark, L.F. (1962). Stimulus coding in the cat's auditory nerve. Preliminary report. Trans. Am. Otol. Soc. 50, 264-283.

Kiang, N.Y., Rho, J.M., Northrop, C.C., Liberman, M.C., and Ryugo, D.K. (1982). Hair-cell innervation by spiral ganglion cells in adult cats. Science 217, 175-177.

Kiang, N.Y.S., Watanabe, T., Thomas, E.C., and Clark, L.F. (1965). Discharge Patterns of Single Fibers in the Cat's Auditory Nerve (Cambridge, Massachusetts: MIT Press).

Kim, M.-H., Li, G.-L., and von Gersdorff, H. (2013). Single Ca2+ channels and exocytosis at sensory synapses. J. Physiol. 591, 3167-3178.

Kjaerulff, O., Brodin, L., and Jung, A. (2011). The structure and function of endophilin proteins. Cell Biochem. Biophys. 60, 137-154.

Klenchin, V.A., and Martin, T.F.J. (2000). Priming in exocytosis: Attaining fusioncompetence after vesicle docking. Biochimie 82, 399-407.

Kononenko, N.L., Puchkov, D., Classen, G.A., Walter, A.M., Pechstein, A., Sawade, L., Kaempf, N., Trimbuch, T., Lorenz, D., Rosenmund, C., et al. (2014). Clathrin/AP-2 mediate synaptic vesicle reformation from endosome-like vacuoles but are not essential for membrane retrieval at central synapses. Neuron $82,981-988$.

Koschak, A., Reimer, D., Huber, I., Grabner, M., Glossmann, H., Engel, J., and Striessnig, J. (2001). alpha 1D (Cav1.3) subunits can form l-type Ca2+ channels activating at negative voltages. J. Biol. Chem. 276, 22100-22106.

Krinner, S., Butola, T., Jung, S., Wichmann, C., and Moser, T. (2017). RIM-Binding Protein 2 Promotes a Large Number of CaV1.3 Ca2+-Channels and Contributes to Fast Synaptic Vesicle Replenishment at Hair Cell Active Zones. Front. Cell. Neurosci. 11, 334.

Kros, C.J., Ruppersberg, J.P., and Rüsch, A. (1998). Expression of a potassium current in inner hair cells during development of hearing in mice. Nature 394, 281-284.

Kuhn, S., Knirsch, M., Rüttiger, L., Kasperek, S., Winter, H., Freichel, M., Flockerzi, V., Knipper, M., and Engel, J. (2009). Ba2+ currents in inner and outer hair cells of mice lacking the voltage-dependent $\mathrm{Ca} 2+$ channel subunits beta3 or beta4. Channels Austin Tex 3, 366376.

Kushmerick, C., Renden, R., and von Gersdorff, H. (2006). Physiological temperatures reduce the rate of vesicle pool depletion and short-term depression via an acceleration of vesicle recruitment. J. Neurosci. 26, 1366.

Liberman, M. (1982). Single-neuron labeling in the cat auditory nerve. Science 216, 12391241.

Liberman, M.C. (1978). Auditory-nerve response from cats raised in a low-noise chamber. J. Acoust. Soc. Am. 63, 442-455.

Liberman, M.C. (1980). Morphological differences among radial afferent fibers in the cat cochlea: an electron-microscopic study of serial sections. Hear. Res. 3, 45-63. 
Liberman, L.D., Wang, H., and Liberman, M.C. (2011). Opposing Gradients of Ribbon Size and AMPA Receptor Expression Underlie Sensitivity Differences among CochlearNerve/Hair-Cell Synapses. J. Neurosci. 31, 801-808.

Lindau, M., and Neher, E. (1988). Patch-clamp techniques for time-resolved capacitance measurements in single cells. Pflüg. Arch. Eur. J. Physiol. 411, 137-146.

Llinás, R., Steinberg, I.Z., and Walton, K. (1981a). Presynaptic calcium currents in squid giant synapse. Biophys. J. 33, 289-321.

Llinás, R., Steinberg, I.Z., and Walton, K. (1981b). Relationship between presynaptic calcium current and postsynaptic potential in squid giant synapse. Biophys. J. 33, 323-351.

Lumpkin, E.A., and Hudspeth, A.J. (1995). Detection of Ca2+ entry through mechanosensitive channels localizes the site of mechanoelectrical transduction in hair cells. Proc. Natl. Acad. Sci. 92, 10297-10301.

van Lunteren, E., Elmslie, K.S., and Jones, S.W. (1993). Effects of temperature on calcium current of bullfrog sympathetic neurons. J. Physiol. 466, 81.

Lv, C., Stewart, W.J., Akanyeti, O., Frederick, C., Zhu, J., Santos-Sacchi, J., Sheets, L., Liao, J.C., and Zenisek, D. (2016). Synaptic Ribbons Require Ribeye for Electron Density, Proper Synaptic Localization, and Recruitment of Calcium Channels. Cell Rep. 15, 2784-2795.

Marcotti, W., Johnson, S.L., Holley, M.C., and Kros, C.J. (2003). Developmental changes in the expression of potassium currents of embryonic, neonatal and mature mouse inner hair cells. J. Physiol. 548, 383-400.

Marcotti, W., Johnson, S.L., and Kros, C.J. (2004). Effects of intracellular stores and extracellular $\mathrm{Ca}(2+)$ on $\mathrm{Ca}(2+)$-activated $\mathrm{K}(+)$ currents in mature mouse inner hair cells. J. Physiol. 557, 613-633.

Matsubara, A., Laake, J.H., Davanger, S., Usami, S., and Ottersen, O.P. (1996). Organization of AMPA receptor subunits at a glutamate synapse: a quantitative immunogold analysis of hair cell synapses in the rat organ of Corti. J. Neurosci. 16, 4457.

Matthews, G., and Sterling, P. (2008). Evidence that vesicles undergo compound fusion on the synaptic ribbon. J. Neurosci. 28, 5403.

Matveev, V., Bertram, R., and Sherman, A. (2009). Ca2+ Current versus Ca2+ Channel Cooperativity of Exocytosis. J. Neurosci. 29, 12196-12209.

Matveev, V., Bertram, R., and Sherman, A. (2011). Calcium cooperativity of exocytosis as a measure of $\mathrm{Ca} 2+$ channel domain overlap. Brain Res. 1398, 126-138.

Maxeiner, S., Luo, F., Tan, A., Schmitz, F., and Südhof, T.C. (2016). How to make a synaptic ribbon: RIBEYE deletion abolishes ribbons in retinal synapses and disrupts neurotransmitter release. EMBO J. 35, 1098-1114.

Mehta, B., Snellman, J., Chen, S., Li, W., and Zenisek, D. (2013). Synaptic Ribbons Influence the Size and Frequency of Miniature-like Evoked Postsynaptic Currents. Neuron $77,516-527$. 
Merchan-Perez, A., and Liberman, M.C. (1996). Ultrastructural differences among afferent synapses on cochlear hair cells: correlations with spontaneous discharge rate. J. Comp. Neurol. 371, 208-221.

Meyer, A.C., and Moser, T. (2010). Structure and function of cochlear afferent innervation. Curr. Opin. Otolaryngol. Head Neck Surg. 18, 441-446.

Michalski, N.A., Goutman, J.D., Auclair, S.M., Monvel, J.B. de, Tertrais, M., Emptoz, A., Parrin, A., Nouaille, S., Guillon, M., Sachse, M., et al. (2017). Otoferlin acts as a Ca2+ sensor for vesicle fusion and vesicle pool replenishment at auditory hair cell ribbon synapses. ELife 6, e31013.

Michanski, S., Smaluch, K., Steyer, A.M., Chakrabarti, R., Setz, C., Oestreicher, D., Fischer, C., Möbius, W., Moser, T., Vogl, C., et al. (2019). Mapping developmental maturation of inner hair cell ribbon synapses in the apical mouse cochlea. Proc. Natl. Acad. Sci. 116, 64156424.

Micheva, K.D., and Smith, S.J. (2005). Strong effects of subphysiological temperature on the function and plasticity of mammalian presynaptic terminals. J. Neurosci. 25, 7481.

Milosevic, I., Giovedi, S., Lou, X., Raimondi, A., Collesi, C., Shen, H., Paradise, S., O'Toole, E., Ferguson, S., Cremona, O., et al. (2011). Recruitment of Endophilin to ClathrinCoated Pit Necks Is Required for Efficient Vesicle Uncoating after Fission. Neuron 72, 587601.

Moser, T., and Beutner, D. (2000). Kinetics of exocytosis and endocytosis at the cochlear inner hair cell afferent synapse of the mouse. Proc. Natl. Acad. Sci. 97, 883-888.

Moser, T., and Vogl, C. (2016). New insights into cochlear sound encoding. F1000Research 5, 2081.

Moser, T., Neef, A., and Khimich, D. (2006a). Mechanisms underlying the temporal precision of sound coding at the inner hair cell ribbon synapse. J. Physiol. 576, 55-62.

Moser, T., Brandt, A., and Lysakowski, A. (2006b). Hair cell ribbon synapses. Cell Tissue Res. 326, 347-359.

Moser, T., Grabner, C.P., and Schmitz, F. (2019). Sensory processing at ribbon synapses in the retina and the cochlea. Physiol. Rev. 100, 103-144.

Müller, M., von Hünerbein, K., Hoidis, S., and Smolders, J.W.T. (2005). A physiological place-frequency map of the cochlea in the CBA/J mouse. Hear. Res. 202, 63-73.

Neef, A., Khimich, D., Pirih, P., Riedel, D., Wolf, F., and Moser, T. (2007). Probing the Mechanism of Exocytosis at the Hair Cell Ribbon Synapse. J. Neurosci. 27, 12933-12944.

Neef, J., Gehrt, A., Bulankina, A.V., Meyer, A.C., Riedel, D., Gregg, R.G., Strenzke, N., and Moser, T. (2009). The Ca2+ Channel Subunit beta2 Regulates Ca2+ Channel Abundance and Function in Inner Hair Cells and Is Required for Hearing. J. Neurosci. 29, 10730 . 
Neef, J., Jung, S., Wong, A.B., Reuter, K., Pangrsic, T., Chakrabarti, R., Kugler, S., Lenz, C., Nouvian, R., Boumil, R.M., et al. (2014). Modes and Regulation of Endocytic Membrane Retrieval in Mouse Auditory Hair Cells. J. Neurosci. 34, 705-716.

Neef, J., Urban, N.T., Ohn, T.-L., Frank, T., Jean, P., Hell, S.W., Willig, K.I., and Moser, T. (2018). Quantitative optical nanophysiology of $\mathrm{Ca} 2+$ signaling at inner hair cell active zones. Nat. Commun. 9, 290.

Neher, E. (1998). Vesicle pools and Ca2+ microdomains: new tools for understanding their roles in neurotransmitter release. Neuron 20, 389-399.

Neher, E., and Sakaba, T. (2008). Multiple roles of calcium ions in the regulation of neurotransmitter release. Neuron 59, 861-872.

Nouvian, R. (2007). Temperature enhances exocytosis efficiency at the mouse inner hair cell ribbon synapse. J. Physiol. 584, 535-542.

Nouvian, R., Neef, J., Bulankina, A.V., Reisinger, E., Pangršič, T., Frank, T., Sikorra, S., Brose, N., Binz, T., and Moser, T. (2011). Exocytosis at the hair cell ribbon synapse apparently operates without neuronal SNARE proteins. Nat. Neurosci. 14, 411-413.

Oesch, N.W., and Diamond, J.S. (2011). Ribbon synapses compute temporal contrast and encode luminance in retinal rod bipolar cells. Nat. Neurosci. 14, 1555-1561.

Oheim, M., Kirchhoff, F., and Stühmer, W. (2006). Calcium microdomains in regulated exocytosis. Cell Calcium 40, 423-439.

Ohlemiller, K.K., and Echteler, S.M. (1990). Functional correlates of characteristic frequency in single cochlear nerve fibers of the Mongolian gerbil. J. Comp. Physiol. [A] 167, 329-338.

Ohlemiller, K.K., Echteler, S.M., and Siegel, J.H. (1991). Factors that influence rate-versusintensity relations in single cochlear nerve fibers of the gerbil. J. Acoust. Soc. Am. 90, 274287.

Ohn, T.-L., Rutherford, M.A., Jing, Z., Jung, S., Duque-Afonso, C.J., Hoch, G., Picher, M.M., Scharinger, A., Strenzke, N., and Moser, T. (2016). Hair cells use active zones with different voltage dependence of $\mathrm{Ca} 2+$ influx to decompose sounds into complementary neural codes. Proc. Natl. Acad. Sci. 113, E4716-E4725.

Oliver, D., Knipper, M., Derst, C., and Fakler, B. (2003). Resting potential and submembrane calcium concentration of inner hair cells in the isolated mouse cochlea are set by KCNQ-type potassium channels. J. Neurosci. Off. J. Soc. Neurosci. 23, 2141-2149.

Paillart, C., Li, J., Matthews, G., and Sterling, P. (2003). Endocytosis and vesicle recycling at a ribbon synapse. J. Neurosci. 23, 4092-4099.

Palmer, A.R., and Russell, I.J. (1986). Phase-locking in the cochlear nerve of the guinea-pig and its relation to the receptor potential of inner hair-cells. Hear. Res. 24, 1-15.

Palmer, M.J., Hull, C., Vigh, J., and von Gersdorff, H. (2003). Synaptic Cleft Acidification and Modulation of Short-Term Depression by Exocytosed Protons in Retinal Bipolar Cells. J. Neurosci. 23, 11332-11341. 
Pangrsic, T., Lasarow, L., Reuter, K., Takago, H., Schwander, M., Riedel, D., Frank, T., Tarantino, L.M., Bailey, J.S., Strenzke, N., et al. (2010). Hearing requires otoferlindependent efficient replenishment of synaptic vesicles in hair cells. Nat. Neurosci. 13, 869876.

Pangršič, T., Reisinger, E., and Moser, T. (2012). Otoferlin: a multi-C2 domain protein essential for hearing. Trends Neurosci. 35, 671-680.

Pangršič, T., Gabrielaitis, M., Michanski, S., Schwaller, B., Wolf, F., Strenzke, N., and Moser, T. (2015). EF-hand protein $\mathrm{Ca} 2+$ buffers regulate $\mathrm{Ca} 2+$ influx and exocytosis in sensory hair cells. Proc. Natl. Acad. Sci. 112, E1028-E1037.

Pangrsic, T., Singer, J.H., and Koschak, A. (2018). Voltage-Gated Calcium Channels: Key Players in Sensory Coding in the Retina and the Inner Ear. Physiol. Rev. 98, 2063-2096.

Parks, T.N. (2000). The AMPA receptors of auditory neurons. Hear. Res. 147, 77-91.

Peterson, A.J., Irvine, D.R.F., and Heil, P. (2014). A model of synaptic vesicle-pool depletion and replenishment can account for the interspike interval distributions and nonrenewal properties of spontaneous spike trains of auditory-nerve fibers. J. Neurosci. Off. J. Soc. Neurosci. 34, 15097-15109.

Petitpré, C., Wu, H., Sharma, A., Tokarska, A., Fontanet, P., Wang, Y., Helmbacher, F., Yackle, K., Silberberg, G., Hadjab, S., et al. (2018). Neuronal heterogeneity and stereotyped connectivity in the auditory afferent system. Nat. Commun. 9, 3691.

Picher, M.M., Gehrt, A., Meese, S., Ivanovic, A., Predoehl, F., Jung, S., Schrauwen, I., Dragonetti, A.G., Colombo, R., Camp, G.V., et al. (2017a). Ca2+-binding protein 2 inhibits Ca2+-channel inactivation in mouse inner hair cells. Proc. Natl. Acad. Sci. 114, E1717E1726.

Picher, M.M., Oprişoreanu, A.-M., Jung, S., Michel, K., Schoch, S., and Moser, T. (2017b). Rab Interacting Molecules 2 and 3 Directly Interact with the Pore-Forming CaV1.3 Ca2+ Channel Subunit and Promote Its Membrane Expression. Front. Cell. Neurosci. 11.

Pickles, J.O., Comis, S.D., and Osborne, M.P. (1984). Cross-links between stereocilia in the guinea pig organ of Corti, and their possible relation to sensory transduction. Hear. Res. 15, $103-112$.

Platzer, J., Engel, J., Schrott-Fischer, A., Stephan, K., Bova, S., Chen, H., Zheng, H., and Striessnig, J. (2000). Congenital deafness and sinoatrial node dysfunction in mice lacking class D L-type Ca2+ channels. Cell 102, 89-97.

Ramakrishnan, N.A., Drescher, M.J., Morley, B.J., Kelley, P.M., and Drescher, D.G. (2014). Calcium Regulates Molecular Interactions of Otoferlin with SNARE Proteins Required for Hair Cell Exocytosis. J. Biol. Chem.

Ravindranathan, A., Donevan, S.D., Sugden, S.G., Greig, A., Rao, M.S., and Parks, T.N. (2000). Contrasting molecular composition and channel properties of AMPA receptors on chick auditory and brainstem motor neurons. J. Physiol. 523, 667-684.

Regus-Leidig, H., Fuchs, M., Löhner, M., Leist, S.R., Leal-Ortiz, S., Chiodo, V.A., Hauswirth, W.W., Garner, C.C., and Brandstätter, J.H. (2014). In vivo knockdown of 
Piccolino disrupts presynaptic ribbon morphology in mouse photoreceptor synapses. Front. Cell. Neurosci. 8, 259.

Reisinger, E., Bresee, C., Neef, J., Nair, R., Reuter, K., Bulankina, A., Nouvian, R., Koch, M., Bückers, J., Kastrup, L., et al. (2011). Probing the functional equivalence of otoferlin and synaptotagmin 1 in exocytosis. J. Neurosci. 31, 4886-4895.

Relkin, E.M., and Doucet, J.R. (1991). Recovery from prior stimulation. I: Relationship to spontaneous firing rates of primary auditory neurons. Hear. Res. 55, 215-222.

Renden, R., and von Gersdorff, H. (2007). Synaptic Vesicle Endocytosis at a CNS Nerve Terminal: Faster Kinetics at Physiological Temperatures and Increased Endocytotic Capacity During Maturation. J. Neurophysiol. 98, 3349-3359.

Reutens, A.T., and Begley, C.G. (2002). Endophilin-1: a multifunctional protein. Int. J. Biochem. Cell Biol. 34, 1173-1177.

Revelo, N.H., Kamin, D., Truckenbrodt, S., Wong, A.B., Reuter-Jessen, K., Reisinger, E., Moser, T., and Rizzoli, S.O. (2014). A new probe for super-resolution imaging of membranes elucidates trafficking pathways. J. Cell Biol. 205, 591-606.

Rhode, W.S., and Smith, P.H. (1985). Characteristics of tone-pip response patterns in relationship to spontaneous rate in cat auditory nerve fibers. Hear. Res. 18, 159-168.

Roberts, W.M., Jacobs, R.A., and Hudspeth, A.J. (1990). Colocalization of ion channels involved in frequency selectivity and synaptic transmission at presynaptic active zones of hair cells. J. Neurosci. Off. J. Soc. Neurosci. 10, 3664-3684.

Robertson, D. (1984). Horseradish peroxidase injection of physiologically characterized afferent and efferent neurones in the guinea pig spiral ganglion. Hear. Res. 15, 113-121.

Robertson, D., and Paki, B. (2002). Role of L-Type Ca2+ Channels in Transmitter Release From Mammalian Inner Hair Cells. II. Single-Neuron Activity. J. Neurophysiol. 87, 27342740 .

Romand, R. (1984). Functional properties of auditory-nerve fibers during postnatal development in the kitten. Exp. Brain Res. 56, 395-402.

Rose, J.E., Brugge, J.F., Anderson, D.J., and Hind, J.E. (1967). Phase-locked response to low-frequency tones in single auditory nerve fibers of the squirrel monkey. J. Neurophysiol. 30, 769-793.

Rose, J.E., Hind, J.E., Anderson, D.J., and Brugge, J.F. (1971). Some effects of stimulus intensity on response of auditory nerve fibers in the squirrel monkey. J. Neurophysiol. 34, 685-699.

Roux, I., Safieddine, S., Nouvian, R., Grati, M., Simmler, M.-C., Bahloul, A., Perfettini, I., Le Gall, M., Rostaing, P., Hamard, G., et al. (2006). Otoferlin, defective in a human deafness form, is essential for exocytosis at the auditory ribbon synapse. Cell 127, 277-289.

Rudolph, S., Tsai, M.-C., von Gersdorff, H., and Wadiche, J.I. (2015). The ubiquitous nature of multivesicular release. Trends Neurosci. 38, 428-438. 
Ruel, J., Chen, C., Pujol, R., Bobbin, R.P., and Puel, J.L. (1999). AMPA-preferring glutamate receptors in cochlear physiology of adult guinea-pig. J. Physiol. 518, 667-680.

Russell, I.J. (1983). Origin of the receptor potential in inner hair cells of the mammalian cochlea - Evidence for Davis' theory. Nature.

Russell, I.J., and Sellick, P.M. (1978). Intracellular studies of hair cells in the mammalian cochlea. J. Physiol. 284, 261-290.

Rutherford, M.A., and Moser, T. (2016). The Ribbon Synapse Between Type I Spiral Ganglion Neurons and Inner Hair Cells. In The Primary Auditory Neurons of the Mammalian Cochlea, A. Dabdoub, B. Fritzsch, A.N. Popper, and R.R. Fay, eds. (New York, NY: Springer New York), pp. 117-156.

Rutherford, M.A., Chapochnikov, N.M., and Moser, T. (2012). Spike Encoding of Neurotransmitter Release Timing by Spiral Ganglion Neurons of the Cochlea. J. Neurosci. $32,4773-4789$.

Sachs, M.B., and Abbas, P.J. (1974). Rate versus level functions for auditory-nerve fibers in cats: tone-burst stimuli. J. Acoust. Soc. Am. 56, 1835-1847.

Sachs, M.B., Winslow, R.L., and Sokolowski, B.H. (1989). A computational model for ratelevel functions from cat auditory-nerve fibers. Hear. Res. 41, 61-69.

Safieddine, S., and Wenthold, R.J. (1999). SNARE complex at the ribbon synapses of cochlear hair cells: analysis of synaptic vesicle- and synaptic membrane-associated proteins. Eur. J. Neurosci. 11, 803-812.

Safieddine, S., El-Amraoui, A., and Petit, C. (2012). The auditory hair cell ribbon synapse: from assembly to function. Annu. Rev. Neurosci. 35, 509-528.

Scharinger, A., Eckrich, S., Vandael, D.H., Schönig, K., Koschak, A., Hecker, D., Kaur, G., Lee, A., Sah, A., Bartsch, D., et al. (2015). Cell-type-specific tuning of Cav1.3 Ca(2+)channels by a C-terminal automodulatory domain. Front. Cell. Neurosci. 9, 309.

Schmiedt, R.A. (1989). Spontaneous rates, thresholds and tuning of auditory-nerve fibers in the gerbil: comparisons to cat data. Hear. Res. 42, 23-35.

Schmitz, F., Königstorfer, A., and Südhof, T.C. (2000). RIBEYE, a component of synaptic ribbons: a protein's journey through evolution provides insight into synaptic ribbon function. Neuron 28, 857-872.

Schnee, M.E., Lawton, D.M., Furness, D.N., Benke, T.A., and Ricci, A.J. (2005). Auditory hair cell-afferent fiber synapses are specialized to operate at their best frequencies. Neuron $47,243-254$.

Schnee, M.E., Castellano-Muñoz, M., and Ricci, A.J. (2013). Response properties from turtle auditory hair cell afferent fibers suggest spike generation is driven by synchronized release both between and within synapses. J. Neurophysiol. 110, 204-220.

Schrauwen, I., Helfmann, S., Inagaki, A., Predoehl, F., Tabatabaiefar, M.A., Picher, M.M., Sommen, M., Seco, C.Z., Oostrik, J., Kremer, H., et al. (2012). A Mutation in CABP2, 
Expressed in Cochlear Hair Cells, Causes Autosomal-Recessive Hearing Impairment. Am. J. Hum. Genet. 91, 636-645.

Scimemi, A., and Diamond, J.S. (2012). The number and organization of Ca2+ channels in the active zone shapes neurotransmitter release from Schaffer collateral synapses. J. Neurosci. Off. J. Soc. Neurosci. 32, 18157-18176.

Sewell, W.F. (1984). The relation between the endocochlear potential and spontaneous activity in auditory nerve fibres of the cat. J. Physiol. 347, 685-696.

Sheets, L., Trapani, J.G., Mo, W., Obholzer, N., and Nicolson, T. (2011). Ribeye is required for presynaptic $\mathrm{CaV} 1.3$ a channel localization and afferent innervation of sensory hair cells. Development 138, 1309-1319.

Sheets, L., He, X.J., Olt, J., Schreck, M., Petralia, R.S., Wang, Y.-X., Zhang, Q., Beirl, A., Nicolson, T., Marcotti, W., et al. (2017). Enlargement of Ribbons in Zebrafish Hair Cells Increases Calcium Currents But Disrupts Afferent Spontaneous Activity and Timing of Stimulus Onset. J. Neurosci. 37, 6299-6313.

Shen, Y., Yu, D., Hiel, H., Liao, P., Yue, D.T., Fuchs, P.A., and Soong, T.W. (2006). Alternative splicing of the $\mathrm{Ca}(\mathrm{v}) 1.3$ channel IQ domain, a molecular switch for $\mathrm{Ca} 2+-$ dependent inactivation within auditory hair cells. J. Neurosci. 26, 10690-10699.

Sheng, J., He, L., Zheng, H., Xue, L., Luo, F., Shin, W., Sun, T., Kuner, T., Yue, D.T., and $\mathrm{Wu}$, L.-G. (2012). Calcium-channel number critically influences synaptic strength and plasticity at the active zone. Nat. Neurosci. 15, 998-1006.

Shrestha, B.R., Chia, C., Wu, L., Kujawa, S.G., Liberman, M.C., and Goodrich, L.V. (2018). Sensory Neuron Diversity in the Inner Ear Is Shaped by Activity. Cell 174, 1229-1246.e17.

Siegel, J.H. (1992). Spontaneous synaptic potentials from afferent terminals in the guinea pig cochlea. Hear. Res. 59, 85-92.

Siemens, J., Lillo, C., Dumont, R.A., Reynolds, A., Williams, D.S., Gillespie, P.G., and Müller, U. (2004). Cadherin 23 is a component of the tip link in hair-cell stereocilia. Nature $428,950-955$.

Singh, A., Gebhart, M., Fritsch, R., Sinnegger-Brauns, M.J., Poggiani, C., Hoda, J.-C., Engel, J., Romanin, C., Striessnig, J., and Koschak, A. (2008). Modulation of Voltage- and $\mathrm{Ca} 2+$-dependent Gating of CaV1.3 L-type Calcium Channels by Alternative Splicing of a C-terminal Regulatory Domain. J. Biol. Chem. 283, 20733-20744.

Snellman, J., Mehta, B., Babai, N., Bartoletti, T.M., Akmentin, W., Francis, A., Matthews, G., Thoreson, W., and Zenisek, D. (2011). Acute destruction of the synaptic ribbon reveals a role for the ribbon in vesicle priming. Nat. Neurosci. 14, 1135-1141.

Söllner, C., Rauch, G.-J., Siemens, J., Geisler, R., Schuster, S.C., Müller, U., Nicolson, T., and Tübingen 2000 Screen Consortium (2004). Mutations in cadherin 23 affect tip links in zebrafish sensory hair cells. Nature 428, 955-959.

Spassova, M., Saunders, J.C., and Parsons, T.D. (2001). Chick cochlear hair cell exocytosis mediated by dihydropyridine-sensitive calcium channels. J. Physiol. 535, 689-696. 
Spassova, M.A., Avissar, M., Furman, A.C., Crumling, M.A., Saunders, J.C., and Parsons, T.D. (2004). Evidence that rapid vesicle replenishment of the synaptic ribbon mediates recovery from short-term adaptation at the hair cell afferent synapse. J. Assoc. Res. Otolaryngol. JARO 5, 376-390.

Spoendlin, H. (1969). Innervation patterns in the organ of corti of the cat. Acta Otolaryngol. (Stockh.) 67, 239-254.

Spoendlin, H. (1972). Innervation densities of the cochlea. Acta Otolaryngol. (Stockh.) 73, $235-248$.

Strenzke, N., Chanda, S., Kopp-Scheinpflug, C., Khimich, D., Reim, K., Bulankina, A.V., Neef, A., Wolf, F., Brose, N., Xu-Friedman, M.A., et al. (2009). Complexin-I Is Required for High-Fidelity Transmission at the Endbulb of Held Auditory Synapse. J. Neurosci. 29, 7991-8004.

Strenzke, N., Chakrabarti, R., Al-Moyed, H., Müller, A., Hoch, G., Pangrsic, T., Yamanbaeva, G., Lenz, C., Pan, K.-T., Auge, E., et al. (2016). Hair cell synaptic dysfunction, auditory fatigue and thermal sensitivity in otoferlin Ile515Thr mutants. EMBO J. 35, e201694564.

Sumner, C.J., and Palmer, A.R. (2012). Auditory nerve fibre responses in the ferret. Eur. J. Neurosci. 36, 2428-2439.

Sun, S., Babola, T., Pregernig, G., So, K.S., Nguyen, M., Su, S.-S.M., Palermo, A.T., Bergles, D.E., Burns, J.C., and Müller, U. (2018). Hair Cell Mechanotransduction Regulates Spontaneous Activity and Spiral Ganglion Subtype Specification in the Auditory System. Cell 174, 1247-1263.e15.

Taberner, A.M., and Liberman, M.C. (2005). Response Properties of Single Auditory Nerve Fibers in the Mouse. J. Neurophysiol. 93, 557-569.

Takamori, S. (2016). Presynaptic Molecular Determinants of Quantal Size. Front. Synaptic Neurosci. 8.

Tan, B.Z., Jiang, F., Tan, M.Y., Yu, D., Huang, H., Shen, Y., and Soong, T.W. (2011). Functional characterization of alternative splicing in the $\mathrm{C}$ terminus of L-type CaV1.3 channels. J. Biol. Chem. 286, 42725-42735.

Temchin, A.N., Rich, N.C., and Ruggero, M.A. (2008). Threshold Tuning Curves of Chinchilla Auditory-Nerve Fibers. I. Dependence on Characteristic Frequency and Relation to the Magnitudes of Cochlear Vibrations. J. Neurophysiol. 100, 2889-2898.

Trapani, J.G., Obholzer, N., Mo, W., Brockerhoff, S.E., and Nicolson, T. (2009). Synaptojanin 1 is required for temporal fidelity of synaptic transmission in hair cells. PLoS Genet. 5, e1000480.

Tsuji, J., and Liberman, M.C. (1997). Intracellular labeling of auditory nerve fibers in guinea pig: central and peripheral projections. J. Comp. Neurol. 381, 188-202.

Vincent, P.F.Y., Bouleau, Y., Charpentier, G., Emptoz, A., Safieddine, S., Petit, C., and Dulon, D. (2017). Different CaV1.3 Channel Isoforms Control Distinct Components of the 
Synaptic Vesicle Cycle in Auditory Inner Hair Cells. J. Neurosci. Off. J. Soc. Neurosci. 37, 2960-2975.

Vincent, P.F.Y., Cho, S., Tertrais, M., Bouleau, Y., von Gersdorff, H., and Dulon, D. (2018). Clustered Ca2+ Channels Are Blocked by Synaptic Vesicle Proton Release at Mammalian Auditory Ribbon Synapses. Cell Rep. 25, 3451-3464.e3.

Vogl, C., Cooper, B.H., Neef, J., Wojcik, S.M., Reim, K., Reisinger, E., Brose, N., Rhee, J.S., Moser, T., and Wichmann, C. (2015). Unconventional molecular regulation of synaptic vesicle replenishment in cochlear inner hair cells. J. Cell Sci. 128, 638-644.

Vogl, C., Panou, I., Yamanbaeva, G., Wichmann, C., Mangosing, S.J., Vilardi, F., Indzhykulian, A.A., Pangršič, T., Santarelli, R., Rodriguez-Ballesteros, M., et al. (2016). Tryptophan-rich basic protein (WRB) mediates insertion of the tail-anchored protein otoferlin and is required for hair cell exocytosis and hearing. EMBO J. 35, 2536-2552.

Von Bekesy, G. (1956). Current status of theories of hearing. Science 123, 779-783.

Walsh, E.J., and McGee, J. (1987). Postnatal development of auditory nerve and cochlear nucleus neuronal responses in kittens. Hear. Res. 28, 97-116.

Wangemann, P., and Schacht, J. (1996). Homeostatic Mechanisms in the Cochlea. In The Cochlea, P. Dallos, A.N. Popper, and R.R. Fay, eds. (Springer New York), pp. 130-185.

Westerman, L.A., and Smith, R.L. (1984). Rapid and short-term adaptation in auditory nerve responses. Hear. Res. 15, 249-260.

Winegar, B.D., and Lansman, J.B. (1990). Voltage-dependent block by zinc of single calcium channels in mouse myotubes. J. Physiol. 425, 563-578.

Winter, I.M., Robertson, D., and Yates, G.K. (1990). Diversity of characteristic frequency rate-intensity functions in guinea pig auditory nerve fibres. Hear. Res. 45, 191-202.

Wittig, J.H., Jr, and Parsons, T.D. (2008). Synaptic ribbon enables temporal precision of hair cell afferent synapse by increasing the number of readily releasable vesicles: a modeling study. J. Neurophysiol. 100, 1724-1739.

Wong, A.B., Jing, Z., Rutherford, M.A., Frank, T., Strenzke, N., and Moser, T. (2013). Concurrent Maturation of Inner Hair Cell Synaptic Ca2+ Influx and Auditory Nerve Spontaneous Activity around Hearing Onset in Mice. J. Neurosci. 33, 10661-10666.

Wong, A.B., Rutherford, M.A., Gabrielaitis, M., Pangršič, T., Göttfert, F., Frank, T., Michanski, S., Hell, S., Wolf, F., Wichmann, C., et al. (2014). Developmental refinement of hair cell synapses tightens the coupling of Ca2+ influx to exocytosis. EMBO J. 33, 247-264.

Wu, J.S., Young, E.D., and Glowatzki, E. (2016). Maturation of Spontaneous Firing Properties after Hearing Onset in Rat Auditory Nerve Fibers: Spontaneous Rates, Refractoriness, and Interfiber Correlations. J. Neurosci. 36, 10584-10597.

Xu, W., and Lipscombe, D. (2001). Neuronal CaV1.3 $\alpha 1$ L-Type Channels Activate at Relatively Hyperpolarized Membrane Potentials and Are Incompletely Inhibited by Dihydropyridines. J. Neurosci. 21, 5944-5951. 
Yasunaga, S., Grati, M., Chardenoux, S., Smith, T.N., Friedman, T.B., Lalwani, A.K., Wilcox, E.R., and Petit, C. (2000). OTOF Encodes Multiple Long and Short Isoforms: Genetic Evidence That the Long Ones Underlie Recessive Deafness DFNB9. Am. J. Hum. Genet. 67, 591-600.

Yates, G.K., Robertson, D., and Johnstone, B.M. (1985). Very rapid adaptation in the guinea pig auditory nerve. Hear. Res. 17, 1-12.

Zampini, V., Johnson, S.L., Franz, C., Lawrence, N.D., Münkner, S., Engel, J., Knipper, M., Magistretti, J., Masetto, S., and Marcotti, W. (2010). Elementary properties of CaV1.3 $\mathrm{Ca}(2+)$ channels expressed in mouse cochlear inner hair cells. J. Physiol. 588, 187-199.

Zhang, L., Engler, S., Koepcke, L., Steenken, F., and Köppl, C. (2018). Concurrent gradients of ribbon volume and AMPA-receptor patch volume in cochlear afferent synapses on gerbil inner hair cells. Hear. Res. 364, 81-89.

Zheng, J., Shen, W., He, D.Z.Z., Long, K.B., Madison, L.D., and Dallos, P. (2000). Prestin is the motor protein of cochlear outer hair cells. Nature 405, 149-155.

Zhou, Z., and Neher, E. (1993). Mobile and immobile calcium buffers in bovine adrenal chromaffin cells. J. Physiol. 469, 245-273. 


\section{Acknowledgements}

I would like to thank Prof. Dr. Tobias Moser for his support and outstanding mentoring throughout my $\mathrm{PhD}$, for giving me the opportunity to join a laboratory that fulfills my scientific and personal goals and for the wonderful paired recordings. I would like to thank my thesis committee members, Prof. Erwin Neher and Prof. Manfred Lindau for their feedback and their inspiring ideas.

I would like to give special thanks to Dr. Chao-Hua Huang for sharing with me the secrets of bouton patching and to Dr. Jakob Neef for sharing his IgorPro knowledge. This work would not have been possible without them, their patience and help.

Thanks to Sandra Gerke, Christiane Senger-Freitag and Ina Herfort for their outstanding technical assistance, their support and their kindness. Thanks to Gerhard Hoch for help with the laser and to Nadine Dietrich for teaching me how to perform ABR recordings. I would like to thank the animal caretakers from the MPIbpc and the UMG for maintaining the mouse lines and always looking for the animal welfare. I am grateful to Patricia Raeke-Kügler and Susann Müller for keeping my head in my neck. I am further thankful to Michael Hörner, who is no longer with us, to Sandra Drube and the IMPRS Neuroscience Coordination Office for their support.

I would like to thank my collaborators on the projects, particularly Jana Kroll and Phillipe Jean. Thanks to the SFB889 and the Nobel Laurate Erwin Neher Fellowship for the financial support during the PhD.

I am very grateful with all the members of the Institute for Auditory Neuroscience for making everyday a nice memory. Particularly, thanks to Carlos el mijo Duque, David Morenito López, Cristian Setz and Özge Maria Özcete for their support during the most difficult moments, the chocolate times, the inspiring scientific discussions, the memes and all the laughs. I am thankful to my Fassberg girls -Tanvi del Pilar Butola and CH- for the amazing lunch times and the patching sessions. My vocabulary wouldn't be the same without them. Thanks to my Fassberg boys -Haris Alvanos, Habakuk Hain and Chad Grabner- for the discussions full of creativity and alternative points of view. I am very grateful to Christian Vogl for being like an older brother to me. Thanks to Rituparna Chakrabarti, Daniel Keppeler, Alexander Dieter and Fritz Kobe for all the great moments we shared together. 
I would like to give special thanks to Dr. Antoine Huet for sharing with me his passion for science, acroyoga, climbing and life. And to Wanderfish for the space, the coffee and the environment to write this thesis.

Last but not least, I would like to thank my parents and my brothers for showing me how to build life roads. They are the greatest role models in my life. I am very grateful to Luisa Fernanda Barbosa and Thomas Offner for their support, comprehension and laughs all these years.

I wouldn't be where I am without all these wonderful people who have crossed my life. 


\section{Appendix}

The synaptic ribbon is critical for sound encoding at high rates and with temporal precision

Philippe Jean†े, David Lopez de la Morenał’, Susann Michanskił, Lina María Jaime Tobónł $\uparrow$, Rituparna Chakrabarti, Maria Magdalena Picher, Jakob Neef, SangYong Jung, Mehmet Gültas, Stephan Maxeiner, Andreas Neef*, Carolin Wichmann*, Nicola Strenzke*, Chad Grabner*, Tobias Moser*

$\dagger$ These authors contributed equally to the work

eLife 2018;7:e29275.

DOI: https://doi.org/10.7554/eLife.29275 


\section{The synaptic ribbon is critical for sound encoding at high rates and with temporal precision}

\section{Philippe Jean ${ }^{1,2,3,4 \dagger}$, David Lopez de la Morena ${ }^{1,3,4 \dagger}$, Susann Michanski ${ }^{2,4,5,6 \dagger}$, Lina María Jaime Tobón ${ }^{1,2,3,4,7,8 \dagger}$, Rituparna Chakrabartiti,3,4,5,6, Maria Magdalena Picher ${ }^{1,2,4}$, Jakob Neef ${ }^{1,2,4,7,8}$, SangYong Jung ${ }^{1,4,9}$,

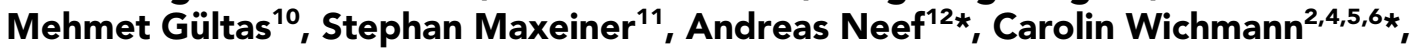 Nicola Strenzke ${ }^{2,4,13 *}$, Chad Grabner ${ }^{1,4,7 *}$, Tobias Moser ${ }^{1,2,4,7,8,14 *}$}

${ }^{1}$ Institute for Auditory Neuroscience and InnerEarLab, University Medical Center Göttingen, Göttingen, Germany; ${ }^{2}$ Collaborative Research Center, University of Göttingen, Göttingen, Germany; ${ }^{3}$ Göttingen Graduate School for Neurosciences and Molecular Biosciences, University of Göttingen, Göttingen, Germany;

${ }^{4}$ InnerEarLab, Department of Otolaryngology, University Medical Center Göttingen, Göttingen, Germany; ${ }^{5}$ Molecular Architecture of Synapses Group, Institute for Auditory Neuroscience, University Medical Center Göttingen, Göttingen, Germany; ${ }^{6}$ Institute for Biostructural Imaging of Neurodegeneration, University Medical Center Göttingen, Göttingen, Germany; ${ }^{7}$ Synaptic Nanophysiology Group, Max Planck Institute for Biophysical Chemistry, Göttingen, Germany; ${ }^{8}$ Auditory Neuroscience Group, Max Planck Institute for Experimental Medicine, Göttingen, Germany; ${ }^{9}$ Neuro Modulation and Neuro Circuitry Group, Singapore Bioimaging Consortium (SBIC), Biomedical Sciences Institutes, Singapore, Singapore; ${ }^{10}$ Department of Breeding Informatics, Georg-August-University Göttingen, Göttingen, Germany; ${ }^{11}$ Institute for Anatomy and Cell Biology, University of the Saarland, Homburg, Germany; ${ }^{12}$ Bernstein Group Biophysics of Neural Computation, Max Planck Institute for Dynamics and Self-Organization, Göttingen, Germany; ${ }^{13}$ Auditory Systems Physiology Group, Department of Otolaryngology, University Medical Center Göttingen, Göttingen, Germany; ${ }^{14}$ Center for Nanoscale Microscopy and Molecular Physiology of the Brain, University Medical Center Göttingen, Göttingen, Germany aneef@gwdg.de (AN); carolin.wichmann@med.unigoettingen.de (CW); nicola.strenzke@med.unigoettingen.de (NS) chad.grabner@mpibpc.mpg.de (CG);

tmoser@gwdg.de (TM)

†These authors contributed equally to this work

Competing interests: The authors declare that no competing interests exist.

Funding: See page 33

Received: 07 July 2017

Accepted: 19 December 2017

Published: 12 January 2018

Reviewing editor: Christine Petit, Institut Pasteur, France

(c) Copyright Jean et al. This article is distributed under the terms of the Creative Commons Attribution License, which permits unrestricted use and redistribution provided that the original author and source are credited.
Abstract We studied the role of the synaptic ribbon for sound encoding at the synapses between inner hair cells (IHCs) and spiral ganglion neurons (SGNs) in mice lacking RIBEYE (RBE ${ }^{\mathrm{KO} /}$ $\mathrm{KO}$ ). Electron and immunofluorescence microscopy revealed a lack of synaptic ribbons and an assembly of several small active zones (AZs) at each synaptic contact. Spontaneous and soundevoked firing rates of SGNs and their compound action potential were reduced, indicating impaired transmission at ribbonless IHC-SGN synapses. The temporal precision of sound encoding was impaired and the recovery of SGN-firing from adaptation indicated slowed synaptic vesicle (SV) replenishment. Activation of $\mathrm{Ca}^{2+}$-channels was shifted to more depolarized potentials and exocytosis was reduced for weak depolarizations. Presynaptic $\mathrm{Ca}^{2+}$-signals showed a broader spread, compatible with the altered $\mathrm{Ca}^{2+}$-channel clustering observed by super-resolution immunofluorescence microscopy. We postulate that RIBEYE disruption is partially compensated by multi-AZ organization. The remaining synaptic deficit indicates ribbon function in SV-replenishment and $\mathrm{Ca}^{2+}$-channel regulation. 


\section{Introduction}

Encoding and processing of sensory information in the ear and the eye rely on ribbon synapses. Described in the 1960s as an electron dense structure tethering a halo of vesicles (Sjostrand, 1958; Smith and Sjostrand, 1961), the function of the synaptic ribbon has remained enigmatic despite decades of work (recent reviews in Lagnado and Schmitz, 2015; Moser and Vogl, 2016; Safieddine et al., 2012; Wichmann and Moser, 2015). Approaches to ribbon function included studies that employed natural variation of ribbon size or abundance during diurnal cycle or hibernation (Hull et al., 2006; Mehta et al., 2013), photoablation (Mehta et al., 2013; Snellman et al., 2011) and genetic manipulation (Dick et alı, 2003; Frank et alı, 2010; Jing et al., 2013; Khimich et al., 2005; Lv et al., 2016; Maxeiner et al., 2016; Sheets et al., 2011; Van Epps et al., 2004). Mutations initially focused on the presynaptic scaffold protein bassoon that is required for ribbon anchorage to the AZ (Dick et al., 2003; Khimich et al., 2005) via interaction with RIBEYE (tom Dieck et al., 2005). However, bassoon also exerts direct effects on AZ function (Davydova et alı, 2014; Hallermann et alı, 2010; Mendoza Schulz et alı, 2014) and, hence, distinguishing direct effects of bassoon deletion and those caused by ribbon loss remained challenging (Jing et al., 2013).

RIBEYE-disruption turned out to be difficult: it is transcribed from the same gene as CtBP2, an essential transcription factor, disruption of which causes embryonic lethality (Hildebrand and Soriano, 2002). Complete abolition of RIBEYE was hard to achieve in zebrafish (Lv et al., 2016; Van Epps et al., 2004) given their duplicated genome. In fact, despite targeting both ribeye genes, RIBEYE immunofluorescence remained present in the retina and hair cells displayed 'ghost ribbons': structures recognized by a synaptic vesicle-halo but lacking electron density (Lv et al., 2016). Complete disruption of RIBEYE expression and lack of retinal ribbons were recently reported in a mouse knock-out of the RIBEYE-specific exon (Maxeiner et al., 2016). This study proved that RIBEYE is required for ribbon formation in the mammalian retina and the observed ribbon loss grossly impaired glutamate release from bipolar cell terminals. The key conclusion was that ribbons help to couple voltage-gated $\mathrm{Ca}^{2+}$-channels to vesicular release sites to enable tight, so-called $\mathrm{Ca}^{2+}$-nanodomain control of exocytosis (Maxeiner et al., 2016), that was previously reported for ribbon synapses of ear and eye (Bartoletti et al., 2011; Brandt et al., 2005; Graydon et al., 2011; Jarsky et al., 2010; Johnson et al., 2017; Pangršič et al., 2015; Wong et al., 2014). By employing the most specific, yet chronic, manipulation of the ribbon, this functional study on ribbonless retinal rod bipolar cells also confirmed that RIBEYE/the ribbon promotes a large complement of vesicular release sites. However, the electrophysiology was performed on rod bipolar cells while the molecular anatomy (immunofluorescence) focused on rod photoreceptors. Since the structure and function of ribbons formed at these two different cell types are distinct, a simple structure-function model was not easy to derive from this study. Moreover, the consequences of ribbon loss remained to be investigated at the systems level. Here, we studied the effects of RIBEYE-disruption on synaptic sound encoding in the cochlea. Combining assessments of the molecular anatomy from electron and fluorescence microscopy with cell and systems physiology, we revealed a role for the synaptic ribbon in organizing the topography of the IHC AZ, in $\mathrm{Ca}^{2+}$-channel regulation and in vesicle replenishment. In summary, we demonstrate that the synaptic ribbon is important for sound encoding at high rates and with temporal precision at IHC synapses.

\section{Results}

\section{Genetic disruption of RIBEYE transforms ribbon-type AZs of IHC synapses into synaptic contacts with multiple small ribbonless $A Z s$}

We first employed immunohistochemistry to study IHCs of 3-week-old RIBEYE knock-out mice $\left(\mathrm{RBE}^{\mathrm{KO} / \mathrm{KO}}\right)$, in which the unique A-domain exon of RIBEYE was deleted by Cre-mediated excision (described in Maxeiner et al., 2016). Next to the A-domain, RIBEYE contains a B-domain that is largely identical to the transcription factor CtBP2, which is spared by the genetic manipulation and used as a target in immunohistochemistry of ribbons and nuclei (Figure 1A,B; Khimich et al., 2005). 
eLife digest Our sense of hearing relies on our ears quickly and tirelessly processing information in a precise manner. Sounds cause vibrations in a part of the inner ear called the cochlea. Inside the cochlea, the vibrations move hair-like structures on sensory cells that translate these movements into electrical signals. These hair cells are connected to specialized nerve cells that relay the signals to the brain, which then interprets them as sounds.

Hair cells communicate with the specialized nerve cells via connections known as chemical synapses. This means that the electrical signals in the hair cell activate channel proteins that allow calcium ions to flow in. This in turn triggers membrane-bound packages called vesicles inside the hair cell to fuse with its surface membrane and release their contents to the outside. The contents, namely chemicals called neurotransmitters, then travels across the space between the cells, relaying the signal to the nerve cell.

The junctions between the hair cells and the nerve cells are more specifically known as ribbon synapses. This is because they have a ribbon-like structure that appears to tether a halo of vesicles close to the active zone where neurotransmitters are released. However, the exact role of this synaptic ribbon has remained mysterious despite decades of study.

The ribbon is mainly composed of a protein called Ribeye, and now Jean, Lopez de la Morena, Michanski, Jaime Tobon et al. show that mutant mice that lack this protein do not have any ribbons at their "ribbon synapses". Hair cells without synaptic ribbons are less able to timely and reliably send signals to the nerve cells, most likely because they cannot replenish the vesicles at the synapse quickly enough. Further analysis showed that the synaptic ribbon also helps to regulate the calcium channels at the synapse, which is important for linking the electrical signals in the hair cell to the release of the neurotransmitters.

Jean et al. also saw that hair cells without ribbons reorganize their synapses to form multiple active zones that could transfer neurotransmitter to the nerve cells. This could partially compensate for the loss of the ribbons, meaning the impact of their loss may have been underestimated. Future studies could explore this by eliminating the Ribeye protein only after the ribbon synapses are fully formed.

These findings may help scientists to better understand deafness and other hearing disorders in humans. They will also be of interest to neuroscientists who research synapses, hearing and other sensory processes.

DOI: https://doi.org/10.7554/eLife.29275.002

Synaptic ribbons of IHC afferent synapses were identified as presynaptic RIBEYE/CtBP2-immunofluorescent spots in wild-type (Figure $1 \mathrm{~B}, \mathrm{RBE}^{\mathrm{WT} / \mathrm{WT}}$ ) and heterozygous (Figure 1-figure supplement 1, RBE ${ }^{\mathrm{WT} / \mathrm{KO}}$ ) mice. Their number per IHC did not change in the heterozygous condition $(15.5 \pm 0.7$, S.D. $=1.58 ; n=50$ cells, $\mathrm{N}=4$ for $\mathrm{RBE}^{\mathrm{WT} / \mathrm{KO}}$ vs. $15.7 \pm 1.1$, S.D. $=2.19 ; n=39$ cells, $\mathrm{N}=3$ for $\mathrm{RBE}^{\mathrm{WT} / \mathrm{WT}}$ at P21), while their intensity was significantly reduced (in arbitrary units: $3.4 \pm 0.7, \mathrm{~S}$. D. $=1.78 ; n=600$ spots for 40 cells, $N=3$ in RBE ${ }^{\mathrm{WT} / \mathrm{KO}}$ vs. $5.1 \pm 1.1$, S.D. $=2.23 ; n=411$ spots for 29 IHCs, $\mathrm{N}=3$ in RBE ${ }^{\mathrm{WT} / W T} ; \mathrm{p}<0.0001$, Mann-Whitney-Wilcoxon test; Figure 1-figure supplement $1 \mathrm{~A}, \mathrm{~B})$. RBE ${ }^{\mathrm{KO} / K O}$ IHCs lacked synaptic RIBEYE/CtBP2 immunofluorescence spots (Figure 1B), while immunolabeling of nuclear CtBP2 remained present, corroborating previous findings in the retina (Maxeiner et al., 2016). The number of afferent synapses per IHC was determined by the count of postsynaptic densities (PSDs) identified as PSD-95 immunofluorescent spots (Figure 1C,D,E) and was unchanged when RIBEYE was removed $\left(13.7 \pm 0.8\right.$, S.D. $=2.04 ; n=56$ cells, $\mathrm{N}=4$ in RBE $\mathrm{KO}^{\mathrm{KO}}$ vs. $12.9 \pm 0.6$, S.D. $=2.13 ; n=55$ cells, $N=5$ in $\mathrm{RBE}^{\mathrm{WT} / \mathrm{WT}}$ ). Bassoon (Figure $1 \mathrm{C}$ ) and RIM2 (Figure 1D), both presynaptic scaffold proteins (Khimich et al., 2005; Jung et al., 2015a), remained present at the ribbonless afferent synapses of $\mathrm{RBE}^{\mathrm{KO} / \mathrm{KO}} \mathrm{IHCs}$ (marked by PSD-95). The scaffold protein piccolino, the short isoform of piccolo (Regus-Leidig et al., 2013) that is present in cochlear and retinal ribbons (Khimich et al., 2005; tom Dieck et al., 2005; Regus-Leidig et al., 2013), was absent from afferent synapses of RBE ${ }^{\mathrm{KO} / \mathrm{KO}} \mathrm{IHCs}$. However, piccolo immunofluorescence was present in the vicinity of afferent synapses likely marking the long form piccolo at the efferent presynaptic AZs (Figure 1E, see schematic in Figure 1A). The PSD areas were calculated by fitting a 2- 


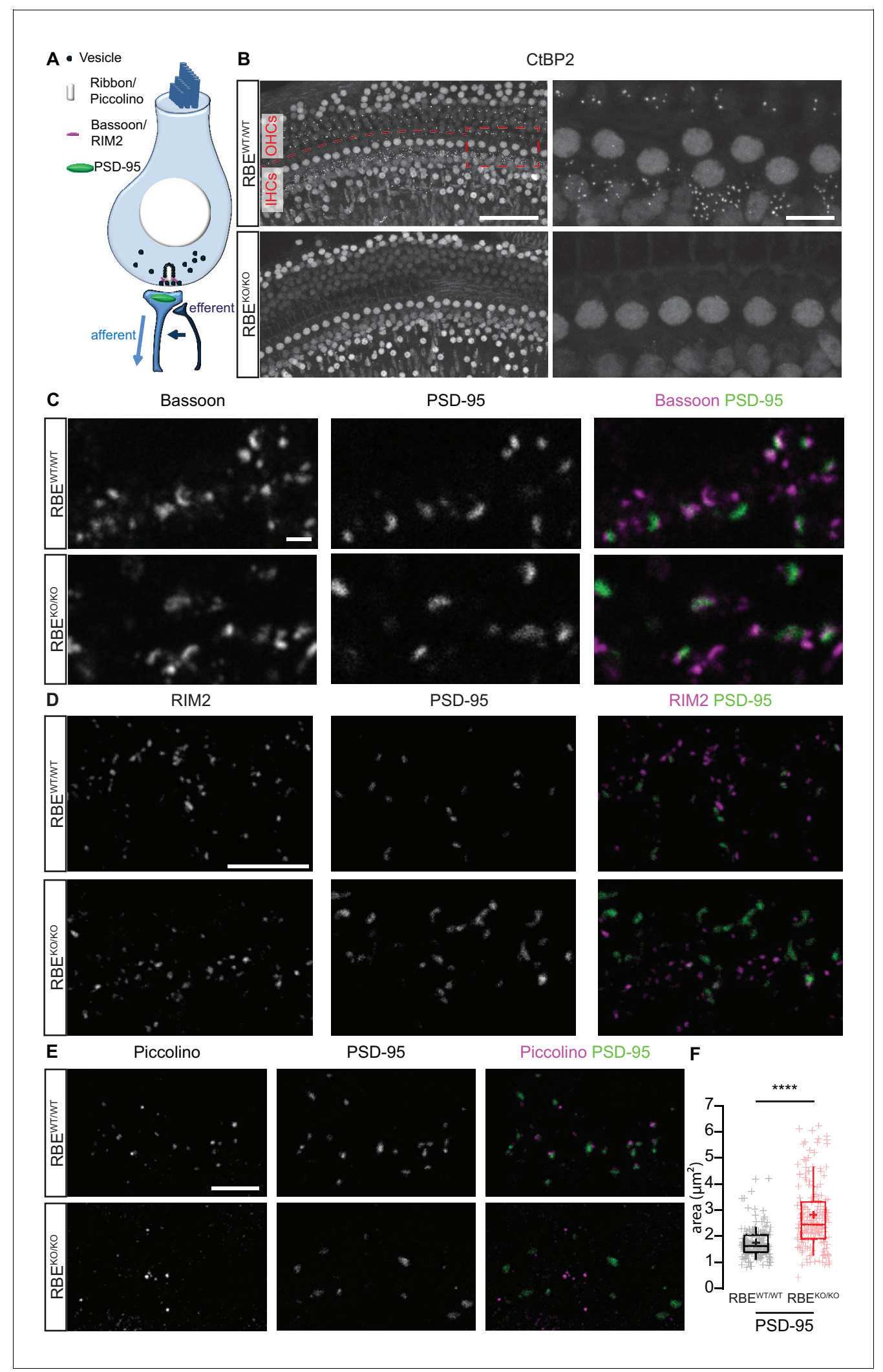

Figure 1. Loss of synaptic ribbons and piccolino from the AZs of RIBEYE-deficient IHCs. (A) Simplified schematic representation of an IHC with the afferent and efferent connectivities. (B) Maximal projection of confocal sections from organs of Corti immunolabeled for CtBP2 and RIBEYE, present in the nuclei and the ribbons, respectively. The RBE ${ }^{W T} / \mathrm{WT}$ staining (top row) shows small puncta in the outer hair cell $(\mathrm{OHC})$ and IHC rows representing the synaptic ribbons, which are completely absent in the RBE ${ }^{\mathrm{KO} / \mathrm{KO}}$ hair cells (bottom row). Scale bar $=50 \mu \mathrm{m}$. Zoom into the IHC row (right column),

Figure 1 continued on next page 
Figure 1 continued

emphasizes the complete disappearance of CtBP2-labeling at the basolateral part of RBE ${ }^{\mathrm{KO} / \mathrm{KO}} \mathrm{IHCs}$. Scale bar $=10 \mu \mathrm{m}$. (C) Maximal projection of confocal sections from organs of Corti co-labeled for the presynaptic marker and anchor of the ribbon, bassoon (left column), and the postsynaptic marker, PSD-95 (middle column), in RBE ${ }^{\mathrm{WT} / \mathrm{WT}}$ and RBE ${ }^{\mathrm{KO} / \mathrm{KO}} \mathrm{HHCs}$. The merged picture (right column) shows the juxtaposition of bassoon (magenta) with PSD-95 (green), indicating its presence both at RBE ${ }^{W T / N T}$ and ribbonless RBE ${ }^{\mathrm{KO} / K O}{ }_{\mathrm{HHC}}$ synapses. Scale bar $=1 \mu \mathrm{m}$. (D) Maximal projection of confocal sections from organs of Corti co-labeled for the presynaptic marker RIM2 (left column) and the postsynaptic marker PSD-95 (middle column). The merged picture (right column) shows the co-localization of RIM2 (magenta) with PSD-95 (green) meaning its presence at the ribbonless IHC presynapses (scale bar $=5 \mu \mathrm{m}$ ). (E) Maximal projection of confocal sections from organs of Corti co-labeled for piccolino, a specific short splice variant of piccolo found at ribbons of RBE ${ }^{\text {WT/WT }}$ IHC synapses (left column), co-labeled with PSD-95 (middle column). The merged pictures (right column) show PSD-95 (green) immunofluorescence lacking juxtaposed piccolino signal (magenta) in RBE ${ }^{\mathrm{KO} / \mathrm{KO}}$ (bottom row), indicating absence of piccolino from afferent synapses of mutant IHCs. The punctate labeling for piccolo, away from PSD-95, most likely represents labeling of piccolo at conventional efferent synapses (schematically shown in Figure 1A). Scale bar $=5 \mu \mathrm{m}$. (F) Quantification of the area of PSD-95 immunofluorescent spots. The PSD-95 spots are siginificantly bigger in the RBE ${ }^{\mathrm{KO} / \mathrm{KO}} \| \mathrm{HCs}\left(\mathrm{p}<0.0001\right.$, Mann-Whitney-Wilcoxon test, $n=178$ spots, $\mathrm{N}=3$ for RBE ${ }^{\mathrm{KO} / \mathrm{KO}}$ and $n=163 \mathrm{spots,}$ $\mathrm{N}=3$ for RBE ${ }^{\mathrm{WT} / \mathrm{NT}}$ ). Box plots show 10, 25, 50, 75 and $90^{\text {th }}$ percentiles with individual data points overlaid; means are shown as crosses. DOI: https://doi.org/10.7554/eLife.29275.003

The following figure supplement is available for figure 1:

Figure supplement 1. Gene-dosage dependent expression of the RIBEYE at IHC AZs.

dimensional Gaussian function to each PSD-95 immunofluorescent spot, revealing a significant increase in the $\mathrm{RBE}^{\mathrm{KO} / \mathrm{KO}}$ condition $\left(2.82 \pm 0.09 \mu \mathrm{m}^{2}\right.$, S.D. $=1.25 ; n=178$ spots, $\mathrm{N}=3$ vs. $1.74 \pm 0.05 \mu \mathrm{m}^{2}$, S.D. $=0.58 ; n=163$ spots, $\mathrm{N}=3$ in RBE ${ }^{\mathrm{WT} / \mathrm{WT}}$ IHCs; $p<0.0001$, Mann-Whitney-Wilcoxon; Figure 1F).

In order to study the effects of RIBEYE deletion on the ultrastructure of afferent IHC synapses, we performed transmission electron microscopy on random sections and electron tomography. Random ultrathin (70-75 nm) sections prepared from P21 mice (two animals per genotype) after aldehyde fixation and conventional embedding procedures showed that IHCs from RBE ${ }^{\mathrm{KO} / \mathrm{KO}}$ mice completely lack synaptic ribbons, while RBE ${ }^{\mathrm{WT} / \mathrm{WT}}$ and heterozygous RBE ${ }^{\mathrm{WT} / K O}$ typically display one ribbon per AZ (Figure 2A-C). Interestingly, ribbons of RBE ${ }^{\mathrm{WT} / K O} \mathrm{IHCs}$ were smaller in height, width and area compared to RBE ${ }^{\mathrm{WT} / \mathrm{WT}} \mathrm{IHC}$ synaptic ribbons (Figure 2-figure supplement 1A-C; ribbon height: $118.32 \pm 3.17 \mathrm{~nm}, \mathrm{~S} . \mathrm{D} .=31.84 \mathrm{~nm} ; n=101$ ribbons, $\mathrm{N}=2$ for $\mathrm{RBE}^{\mathrm{WT} / \mathrm{KO}}$ vs. $197.09 \pm 4.36 \mathrm{~nm}, \mathrm{~S}$. D. $=44.93 \mathrm{~nm} ; n=106$ ribbons, $\mathrm{N}=2$ for $\mathrm{RBE}{ }^{\mathrm{WT}} / \mathrm{WT}$; ribbon width: $119.80 \pm 6.23 \mathrm{~nm}$, S.D. $=62.27$ $\mathrm{nm}$ for $\mathrm{RBE}^{\mathrm{WT} / \mathrm{KO}}$ vs. $168.34 \pm 6.83 \mathrm{~nm}$, S.D. $=70.27 \mathrm{~nm}$ for RBE ${ }^{\mathrm{WT} / \mathrm{WT}}$; ribbon area: $11.5 \mathrm{e} 3 \pm 6.2 \mathrm{e} 2$ $\mathrm{nm}^{2}$, S.D. $=6.3 \mathrm{e} 3 \mathrm{~nm}^{2}$ for RBE ${ }^{\mathrm{WT} / K O}$ vs. $25.4 \mathrm{e} 3 \pm 1.1 \mathrm{e} 2 \mathrm{~nm}^{2}$, S.D. $=1.1 \mathrm{e} 3 \mathrm{~nm}^{2}$ for RBE ${ }^{\mathrm{WT} / W T}$; $p<0.0001$, Mann-Whitney-Wilcoxon test for all) agreeing with the significantly reduced ribbon immunofluorescence intensity in the RBE ${ }^{\mathrm{WT} / \mathrm{KO}}$ condition (see above and Figure 1-figure supplement 1A-B).

Random sections of synaptic contacts of $\mathrm{RBE}^{\mathrm{KO} / \mathrm{KO}}$ mice (Figure $2 \mathrm{C}$ ) often showed more than one presynaptic density (PD), each associated with a cluster of synaptic vesicles (henceforth considered individual AZs). The multiple AZs typically faced one continuous PSD, which is different from the synapses of immature IHC synapses that show multiple appositions of pre- and postsynaptic densities (Sendin et al., 2007; Wong et al., 2014). Moreover, we found more than one PD per synaptic contact in IHCs of older RBE $\mathrm{KO}^{\mathrm{K} O}$ mice (Figure 2E,F; 6 weeks and 8 months, respectively), arguing against a delayed synaptic maturation to be the cause of the phenotype. Sections from tangential cuts of the synapse (Figure 2D), reconstructions from serial ultrathin sections (Figure 2G, $G^{\prime}$ ) and quantifications of random sections (Figure $2 \mathrm{H}$ ) corroborated the notion of multiple small ribbonless AZs at the synaptic contacts of RBE ${ }^{\mathrm{KO} / \mathrm{KO}} \mathrm{IHCs}$. Analysis based on serial 3D reconstructions of synaptic contacts of RBE ${ }^{\mathrm{KO} / \mathrm{KO}} \mathrm{IHC}$ from P21 animals showed on average $1.92 \pm 0.34$ PDs (S.D. = 1.16; $n=17$ serial $3 \mathrm{D}$ reconstructions, $\mathrm{N}=2$ ) and $20.58 \pm 2.98$ total $S V$ s per contact, S.D. $=10.34$ (Figure 2I). The lateral extent of the individual PDs, determined in random sections, was comparable between $\mathrm{RBE}^{\mathrm{KO} / \mathrm{KO}}$ and RBE ${ }^{\mathrm{WT} / \mathrm{WT}}$ synapses $(129.89 \pm 2.53 \mathrm{~nm}, \mathrm{~S} . \mathrm{D} .=26.26 \mathrm{~nm} ; n=108 \mathrm{PDs}, \mathrm{N}=2$ for $\mathrm{RBE}^{\mathrm{KO} / \mathrm{KO}}$ vs. $129.35 \pm 4.89 \mathrm{~nm}$, S.D. $=50.86 \mathrm{~nm} ; n=108 \mathrm{PDs}, \mathrm{N}=2$ for RBE ${ }^{\mathrm{WT} / \mathrm{WT}} ; \mathrm{p}=0.92$, NPMC test), while that of RBEWT/KO was enlarged (Figure $2 M ; 157.64 \pm 7.19 \mathrm{~nm}$, S.D. $=72.24 \mathrm{~nm}$; $n=101$ PDs, $N=2 ; p=0.0004$ for comparison to RBE ${ }^{W T} / W T$, NPMC test). PSDs tended to be increased in length at RBE ${ }^{\mathrm{KO} / K O}$ synapses compared to RBE ${ }^{\mathrm{WT} / \mathrm{WT}} \mathrm{PSD}$ and were significantly larger 

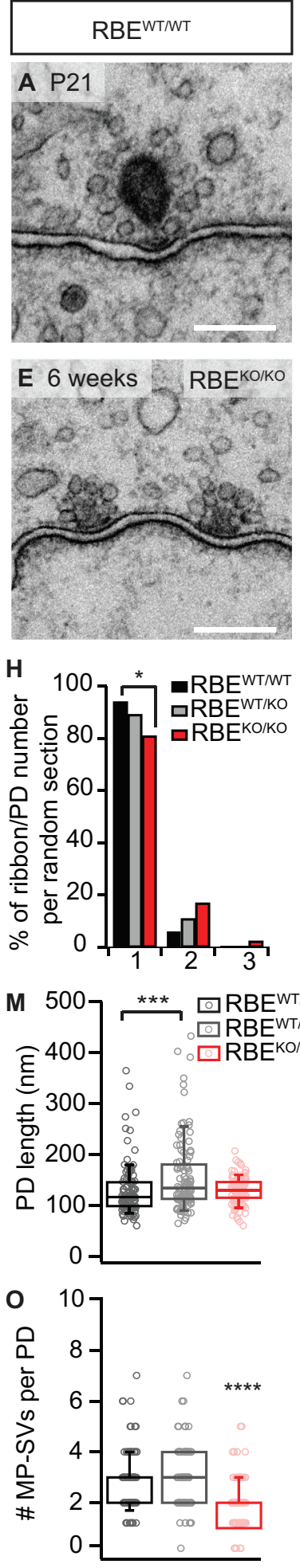
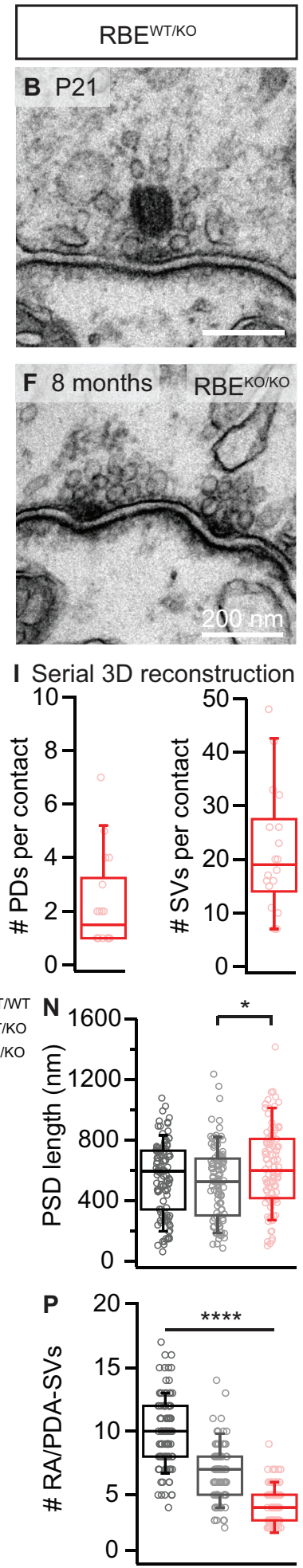
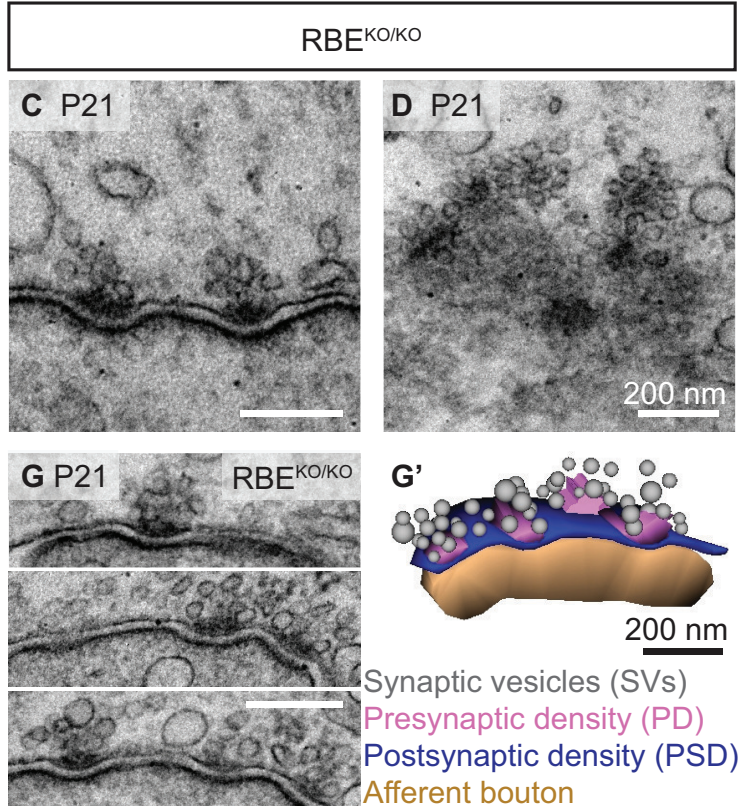

Afferent bouton

J Random section analysis
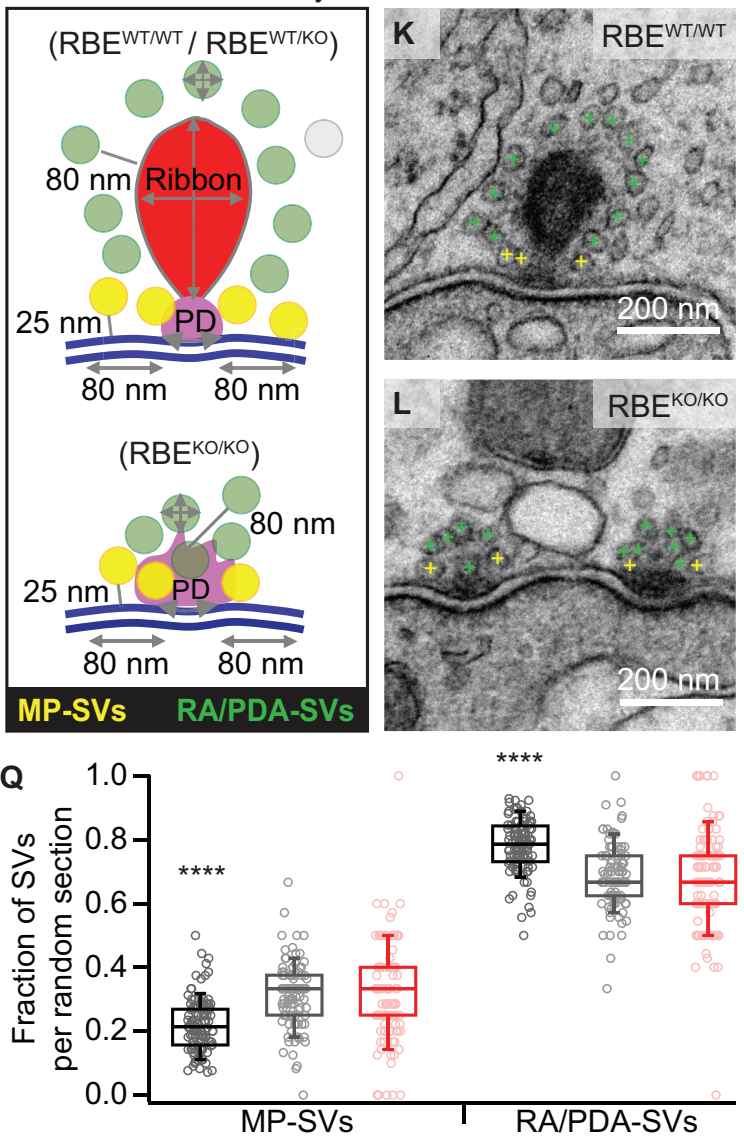

Figure 2. RIBEYE disruption transforms IHC synapses into contacts with multiple small ribbonless AZs. (A-C) Representative electron micrographs of IHC afferent synapses from P21 RBE ${ }^{W T / W T}, \mathrm{RBE}^{\mathrm{WT} / \mathrm{KO}}$ and RBE ${ }^{\mathrm{KO} / \mathrm{KO}}$ mice. Ribbonless RBE ${ }^{\mathrm{KO} / \mathrm{KO}}$ synapses display one or more presynaptic densities (PD) clustering SVs. Scale bars $=200 \mathrm{~nm}$. (D) RBE ${ }^{\mathrm{KO} / \mathrm{KO}} \mathrm{IHC}$ AZ cut tangentially, revealing multiple PDs (here six) per AZ. (E, F) Representative electron micrographs of RBE ${ }^{\mathrm{KO} / \mathrm{KO}} \mathrm{IHC}$ synapses from mice at 6 weeks $(\mathrm{E})$ and 8 months $(\mathrm{F})$ of age: the presence of at least $2 \mathrm{AZs}$ per contact in mature IHCs

Figure 2 continued on next page 
Figure 2 continued

argues against a developmental delay. (G) Consecutive serial sections of a typical RBE ${ }^{\mathrm{KO} / \mathrm{KO}} \mathrm{P} 21 \mathrm{IHC}$ synapse showing multiple $\mathrm{AZs}$. Scale bar $=200$ $\mathrm{nm}$. (G') Corresponding serial 3D reconstruction of the synapse in (G) showing four PDs (magenta) surrounded by a total of 48 SVs (gray). Scale bar $=$ $200 \mathrm{~nm}$. (H) Quantification of the number of ribbon/PD per random section. AZs with a single PD are less frequently observed in RBE ${ }^{\mathrm{KO} / K O}$ IHCs ( $n=108 \mathrm{AZs}, \mathrm{N}=2$ for RBE ${ }^{\mathrm{KO} / \mathrm{KO}}$ and $n=106 \mathrm{AZs}, \mathrm{N}=2$ for RBE ${ }^{\mathrm{WT} / \mathrm{WT}} ; \mathrm{p}<0.05, \mathrm{NPMC}$ test). (I) Number of PDs and SVs per AZ in P21 RBE ${ }^{\mathrm{KO} / \mathrm{KO}}$ mice in serial $3 D$ reconstructions of $R B E^{K O} / K O$ afferent synapses. Box plots show 10, 25, 50, 75 and $90^{\text {th }}$ percentiles with individual data points overlaid, as for ( $M, N, O, P \& Q$ ). (J) Schematic drawing illustrating the quantitative analysis of random sections. SV diameter: average of vertical and horizontal measurements from outer rim to outer rim. The ribbon height, width and area were measured as indicated by the gray lines. The length of the PD was determined along the AZ. For ribbon-occupied AZs: Membrane-proximal (MP) SVs (yellow) were counted in a distance of $\leq 25 \mathrm{~nm}$ from the AZ membrane (blue) and $\leq 80 \mathrm{~nm}$ from the PD. Ribbon-associated (RA) SVs were found in the first layer around the ribbon (red) with a maximum distance of $80 \mathrm{~nm}$ to the ribbon, quantified as indicated by the gray lines. For ribbonless AZs: Instead of RA-SVs we defined PD-associated SVs (PDA-SVs: all SV at PD with a maximum distance of $80 \mathrm{~nm}$ to the PD not matching the MP-SV criteria, defined as above). (K, L) Electron micrographs illustrating the quantification of the MP-SVs (yellow crosses) and the RA/PDA-SVs (green crosses). (M-Q) Quantification of random IHC synapse $\left(\mathrm{P}_{21}\right)$ sections revealed no significant differences between RBE ${ }^{\mathrm{KO} / \mathrm{KO}}$ and RBE ${ }^{\mathrm{WT} / \mathrm{WT}}$ for the PD and PSD length (PD length: $n=108 \mathrm{PDs}, \mathrm{N}=2 \mathrm{for}$ RBE $\mathrm{KO} / \mathrm{KO}$ and $n=108 \mathrm{PDs}$, $N=2$ for RBE ${ }^{W T} / W_{T} ; p=0.92, N P M C$ test and PSD length: $n=98$ PSDs, $N=2$ for RBE KO/KO and $n=113$ PSDs, $N=2$ for RBE ${ }^{W T / W T} ; p=0.11$, Tukey's test). However, in the RBE ${ }^{W T / K O}$ IHCs, the PDs were bigger than in the WT IHCs ( $n=101 \mathrm{PDs}, \mathrm{N}=2 ; \mathrm{p}=0.0004$, NPMC test), and the PSDs were smaller than in the knock-out IHCs ( $n=100$ PSDs, $N=2$ for RBEWT/KO; $p=0.01$, Tukey's test). MP-SVs $\left(n=108\right.$ AZs, $N=2$ for RBE ${ }^{K O} / K O, n=106$ AZs, $N=2$ for $\mathrm{RBE}^{\mathrm{WT} / W T} ; \mathrm{p}<0.0001, \mathrm{NPMC}$ test) and RA/PDA-SVs ( $n=108 \mathrm{AZs}, \mathrm{N}=2$ for RBE ${ }^{\mathrm{KO} / K O}, n=106 \mathrm{AZs}, \mathrm{N}=2$ for RBE ${ }^{\mathrm{WT} / W T} ; \mathrm{p}<0.0001, \mathrm{Tukey}$ 's test) per AZ, as well as the fraction of RA/PDA-SVs in RBE ${ }^{\mathrm{KO} / K O}$, were significantly reduced ( $n=108 \mathrm{AZs}, \mathrm{N}=2$ for RBE ${ }^{\mathrm{KO} / \mathrm{KO}}, n=106 \mathrm{AZs}, \mathrm{N}=2$ for RBE ${ }^{\mathrm{WT} / \mathrm{WT} \text {; }}$ $\mathrm{p}<0.0001, \mathrm{NPMC}$ test).

DOI: https://doi.org/10.7554/eLife.29275.005

The following figure supplement is available for figure 2 :

Figure supplement 1. Random section analysis showed smaller synaptic ribbons and vesicles in RBE ${ }^{\mathrm{WT} / \mathrm{KO}}$ mice. DOI: https://doi.org/10.7554/eLife.29275.006

than RBE ${ }^{\mathrm{WT} / \mathrm{KO}}$ PSDs (Figure $2 \mathrm{~N} ; 623.77 \pm 26.70 \mathrm{~nm}, \mathrm{~S} . \mathrm{D} .=264.33 \mathrm{~nm} ; \mathrm{n}=98$ PSDs, $\mathrm{N}=2$ for $\mathrm{RBE}^{\mathrm{KO} / \mathrm{KO}}$ vs. $555.91 \pm 22.24 \mathrm{~nm}, \mathrm{~S} . \mathrm{D} .=236.42 \mathrm{~nm} ; n=113 \mathrm{PSDs}, \mathrm{N}=2$ for RBE ${ }^{\mathrm{WT} / \mathrm{WT}}$ vs. $521.34 \pm 24.20 \mathrm{~nm}$, S.D. $=242.03 \mathrm{~nm} ; n=100 \mathrm{PSDs}, \mathrm{N}=2$ for RBE ${ }^{\mathrm{WT} / K O} ; \mathrm{p}=0.01$ for $\mathrm{RBE}^{\mathrm{KO} / \mathrm{KO}} \mathrm{vs}$. $\mathrm{RBE}^{\mathrm{WT} / K O}$, Tukey's test), which is consistent with the greater area of PSD-95 immunofluorescent spots in the knock-out condition (Figure 1E).

In the following, we characterized the populations of presynaptic SVs in random sections of vertically-cut IHC synapses. We counted membrane-proximal SVs (MP-SVs, $\leq 25 \mathrm{~nm}$ distance between SV membrane and plasma membrane, laterally within $80 \mathrm{~nm}$ of the PD, yellow in Figure $2 \mathrm{~J}-L$ ) as well as ribbon-associated SVs (RA-SVs, first layer of SVs around the ribbon within $80 \mathrm{~nm}$, green in Figure 2J, $K)$ or 'PD-associated' SVs (PDA-SVs, ribbonless AZs: SVs within $80 \mathrm{~nm}$ distance of the PD and not falling into the MP-SV pool (see above), green in Figure 2J,L). We found both MP-SVs (Figure 2O; $1.92 \pm 0.09$, S.D. $=0.93 ; n=108 \mathrm{AZs}, \mathrm{N}=2$ for RBE ${ }^{\mathrm{KO} / \mathrm{KO}}$ vs. $2.99 \pm 0.12, \mathrm{~S} . \mathrm{D} .=1.18 ; n=101 \mathrm{AZs}$, $\mathrm{N}=2$ for RBE ${ }^{\mathrm{WT} / K O}$ vs. $2.77 \pm 0.12, \mathrm{~S} . \mathrm{D} .=1.18 ; n=106 \mathrm{AZs}, \mathrm{N}=2$ for RBE ${ }^{\mathrm{WT} / \mathrm{WT}} ; \mathrm{p}<0.0001$ for $\mathrm{RBE}^{\mathrm{KO} / \mathrm{KO}}$ vs. RBE ${ }^{\mathrm{WT} / \mathrm{WT}}$, NPMC test) and PDA-SVs (Figure $2 \mathrm{P} ; 4.12 \pm 0.15, \mathrm{~S} . \mathrm{D} .=1.50 ; n=108 \mathrm{AZs}$, $\mathrm{N}=2$ for $\mathrm{RBE}^{\mathrm{KO} / \mathrm{KO}}$ vs. $10.09 \pm 0.27, \mathrm{~S} . \mathrm{D} .=2.75 ; n=106 \mathrm{AZs}, \mathrm{N}=2$ for $\mathrm{RBE}^{\mathrm{WT} / \mathrm{WT}} ; \mathrm{p}<0.0001$, Tukey's test) of the individual ribbonless IHC AZs of $R B E^{K O} / K O$ mice to be significantly fewer than the corresponding number of MP-SVs and RA-SVs counted at RBE ${ }^{W T / W T}$ AZs. The fraction of PDA-SVs relative to the total number of SVs at RBE ${ }^{K O / K O} A Z s$ was less than that of RA-SVs at RBE ${ }^{W T / W T}$ AZs (Figure 2Q; $0.67 \pm 0.02$, S.D. $=0.16 ; n=108 \mathrm{AZs,} N=2$ for $\mathrm{RBE}^{\mathrm{KO} / \mathrm{KO}}$ vs. $0.78 \pm 0.01$, S.D. $=0.08$; $n=106 \mathrm{AZs}, \mathrm{N}=2$ for RBE ${ }^{\mathrm{WT} / \mathrm{WT}} ; \mathrm{p}<0.0001$, NPMC test). Consequently, we observed an increase in the fraction of MP-SVs at RBE ${ }^{\mathrm{KO} / K O} \mathrm{AZs}$ (Figure 2Q; $0.33 \pm 0.02, \mathrm{~S} . \mathrm{D} .=0.16 ; n=108 \mathrm{AZs}, \mathrm{N}=2$ for $\mathrm{RBE}^{\mathrm{KO} / \mathrm{KO}}$ vs. $0.22 \pm 0.01$, S.D. $=0.08 ; n=106 \mathrm{AZs}, \mathrm{N}=2$ for RBE ${ }^{\mathrm{WT} / \mathrm{WT}} ; \mathrm{p}<0.0001, \mathrm{NPMC}$ test). In line with the decreased ribbon size of RBE ${ }^{W T / K O} A Z s$, we found a reduced number of RA-SVs, indicating a hypomorphic phenotype upon the loss of one allele of the RIBEYE gene. The SV diameter was unchanged for all three genotypes when jointly considering SVs of all categories in random sections (Figure 2-figure supplement 1D; $39.59 \pm 0.21 \mathrm{~nm}, \mathrm{~S} . \mathrm{D} .=5.37 \mathrm{~nm} ; \mathrm{n}=108 \mathrm{AZs}, \mathrm{N}=2$ for $\mathrm{RBE}^{\mathrm{KO} / \mathrm{KO}}$ vs. $40.53 \pm 0.14 \mathrm{~nm}$, S.D. $=4.44 \mathrm{~nm} ; n=101 \mathrm{AZs}, \mathrm{N}=2$ for RBE ${ }^{\mathrm{WT} / \mathrm{KO}}$ vs. $41.80 \pm 0.13$ $\mathrm{nm}, \mathrm{S} . \mathrm{D} .=4.79 \mathrm{~nm} ; n=106 \mathrm{AZs}, \mathrm{N}=2$ for RBE ${ }^{\mathrm{WT}} / \mathrm{WT} ; \mathrm{p}=0.30, \mathrm{NPMC}$ test). However, we found a subtle but significant SV-diameter reduction in $\mathrm{RBE}^{\mathrm{KO} / \mathrm{KO}}$ and $\mathrm{RBE} \mathrm{WT}^{\mathrm{K} O}$ for MP-SVs (Figure 2-figure supplement 1E; $39.29 \pm 0.34 \mathrm{~nm}$, S.D. $=4.82 \mathrm{~nm} ; n=108 \mathrm{AZs}, \mathrm{N}=2$ for $\mathrm{RBE}^{\mathrm{KO} / \mathrm{KO}} \mathrm{vs}$. $41.79 \pm 0.26 \mathrm{~nm}, \mathrm{~S} . \mathrm{D} .=4.53 \mathrm{~nm} ; n=106 \mathrm{AZs}, \mathrm{N}=2$ for RBE ${ }^{\mathrm{WT} / \mathrm{WT}} ; \mathrm{p}<0.0001, \mathrm{NPMC}$ test and 
$40.29 \pm 0.25 \mathrm{~nm}$, S.D. $=4.40 \mathrm{~nm} ; n=101 \mathrm{AZs}, \mathrm{N}=2$ for RBE ${ }^{\mathrm{WT} / \mathrm{KO}}$ vs. RBE ${ }^{\mathrm{KO} / \mathrm{KO}} ; \mathrm{p}=0.03, \mathrm{NPMC}$ test) and for RA-/PDA-SVs (Figure 2-figure supplement 1F; $39.72 \pm 0.27 \mathrm{~nm}, \mathrm{~S} . \mathrm{D}$. = $5.61 \mathrm{~nm}$; $n=108 \mathrm{AZs}, \mathrm{N}=2$ for RBE $\mathrm{KO} / \mathrm{KO}$ vs. $41.81 \pm 0.15 \mathrm{~nm}, \mathrm{~S} . \mathrm{D} .=4.86 \mathrm{~nm} ; \mathrm{n}=106 \mathrm{AZs}, \mathrm{N}=2$ for $\mathrm{RBE}^{\mathrm{WT} / \mathrm{WT}} ; \mathrm{p}<0.0001, \mathrm{NPMC}$ test and RBE ${ }^{\mathrm{KO} / \mathrm{KO}}$ vs. $40.63 \pm 0.17 \mathrm{~nm}, \mathrm{~S} . \mathrm{D} .=4.45 \mathrm{~nm} ; \mathrm{n}=101 \mathrm{AZs}$, $\mathrm{N}=2$ for RBE ${ }^{\mathrm{WT} / K O} ; \mathrm{p}=0.003$, NPMC test and RBE ${ }^{\mathrm{WT} / \mathrm{KO}}$ vs. RBE ${ }^{\mathrm{WT} / \mathrm{WT}} ; \mathrm{p}=0.02$, NPMC test).

Next, to capture the synapses in a near-to-native state and to evaluate vesicle tethering, we performed electron tomography on $250 \mathrm{~nm}$ thick sections that were prepared with high-pressure freezing and freeze-substitution (HPF/FS) of organs of Corti from P21 mice (Figure 3). Tomography confirmed the absence of synaptic ribbons and the presence of multiple AZs per contact, each with a clear PD (Figure 3B,D,F). However, we note that the $250 \mathrm{~nm}$ thick sections did typically not fully cover the synaptic contact, which leads to an underestimation for the total number of SVs particularly for the spatially extended RBE ${ }^{\mathrm{KO} / \mathrm{KO}}$ synapses. The PDs appeared roundish in the RBE ${ }^{\mathrm{KO} / \mathrm{KO}}$ with MP-SVs closely arranged around the PD as found at the more elongated RBE ${ }^{\mathrm{WT} / \mathrm{WT}}$ AZs (Figure $3 \mathrm{C}_{\text {, }}$ D). For the tomograms, we followed the definition of MP-SV pool according to the 2D-random sections (Figure 2), but in addition we measured the MP-SVs also in a maximum distance of $50 \mathrm{~nm}$ from the AZ membrane and $\leq 100 \mathrm{~nm}$ from the PD (Figure 3-figure supplement 1 and supplementary file 1). This was motivated by the presence of long tethers connecting SV and AZ membrane and was previously introduced (Jung et alı, 2015a). Further, we distinguished between tethered and non-tethered SVs (Figure 3G, Figure 3-figure supplement 1A). There, we focused our analysis on tethers to the ribbon/PD, plasma membrane and those interconnecting two adjacent SVs (Figure $3 H, I, M, N$ ). We found a significant reduction in the number of MP-SVs per AZ in RBE ${ }^{\mathrm{KO} /}$ $\mathrm{KO}$ IHCs (Figure 3J; RBE ${ }^{\mathrm{KO} / \mathrm{KO}}=6.30 \pm 0.86$, S.D. $=2.87 \mathrm{MP}-\mathrm{SVs} ; n=11 \mathrm{AZs}, \mathrm{N}=3 \mathrm{vs}$. RBE ${ }^{\mathrm{WT} / \mathrm{WT}}=$ $8.70 \pm 0.82$, S.D. $=2.45$ MP-SVs; $n=9$ AZs, $N=3 ; p=0.04$, Mann-Whitney-Wilcoxon test; significant also by the second analysis method: Figure 3-figure supplement 1B, supplementary file 1), while the fraction of tethered MP-SVs (No. of tethered MP-SVs/No. of all MP-SVs) was not significantly altered (Figure $3 \mathrm{~K} ; \mathrm{RBE}^{\mathrm{KO} / \mathrm{KO}}=0.75 \pm 0.07$, S.D. $=0.24 ; n=11 \mathrm{AZs}, \mathrm{N}=3 \mathrm{vs}$. RBE ${ }^{\mathrm{WT} / \mathrm{WT}}=0.65 \pm$ 0.06 , S.D. $=0.18 ; n=9 \mathrm{AZs}, \mathrm{N}=3 ; \mathrm{p}=0.30$, t-test; Figure 3-figure supplement 1C, supplementary file 1). The majority of the MP-SVs were tethered via a single tether in both RBE ${ }^{\mathrm{WT} /}$ WT and $\mathrm{RBE}^{\mathrm{KO} / \mathrm{KO}} \mathrm{IHCs}$. The fraction of MP-SVs with multiple $(\geq 2)$ tethers was significantly larger in $\mathrm{RBE}^{\mathrm{KO} / \mathrm{KO}}$ IHCs (Figure $3 \mathrm{~K}$; single-tethered MP-SVs: $\mathrm{RBE}^{\mathrm{KO} / \mathrm{KO}}=0.55 \pm 0.06, \mathrm{~S} . \mathrm{D} .=0.19 ; n=11$ AZs, $N=3$ vs. RBE ${ }^{\mathrm{WT} / \mathrm{WT}}=0.61 \pm 0.06$, S.D. $=0.17 ; n=9$ AZs, $\mathrm{N}=3 ; \mathrm{p}=0.81$; multiple-tethered MP-SVs: $\mathrm{RBE}^{\mathrm{KO} / \mathrm{KO}}=0.20 \pm 0.05, \mathrm{~S} . \mathrm{D} .=0.15 ; n=11 \mathrm{AZs}, \mathrm{N}=3$ vs. $\mathrm{RBE}^{\mathrm{WT} / \mathrm{WT}}=0.04 \pm 0.02, \mathrm{~S}$. D. $=0.05 ; n=9 \mathrm{AZs}, \mathrm{N}=3 ; \mathrm{p}=0.01$, Tukey's test; Figure 3-figure supplement 1C, supplementary file 1). Further, and in line with analysis of random sections, the number of PDA-SVs

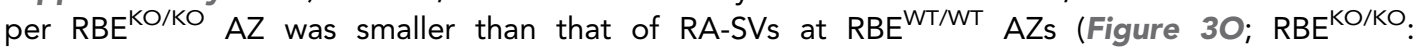
$9.30 \pm 1.13$, S.D. = 3.74 PDA-SVs; $n=11$ AZs, $N=3$ vs. RBE ${ }^{W T / W T}: 30.33 \pm 3.00$, S.D. = 9.01 RA-SVs; $n=9$ AZs, $N=3 ; p<0.0001$, Mann-Whitney-Wilcoxon test; Figure 3-figure supplement 1, supplementary file 1). However, the fraction of PDA-SVs tethered to the PD was not different from that of RA-SVs tethered to the ribbon (Figure 3P; RBE ${ }^{\mathrm{KO} / K O}: 0.80 \pm 0.06, \mathrm{~S} . \mathrm{D} .=0.19$ tethered PDASV fraction; $n=11 \mathrm{AZs}, \mathrm{N}=3$ vs. RBE ${ }^{\mathrm{WT} / \mathrm{WT}}: 0.70 \pm 0.06$, S.D. $=0.17$ tethered RA-SV fraction; $n=9$ $A Z s, N=3 ; p=0.12$, t-test; Figure 3-figure supplement 1F, supplementary file 1).

Finally, tomography indicated unchanged SV diameters at RBE ${ }^{\mathrm{KO} / \mathrm{KO}}$ AZs (Figure 3L, Q; MP-SV diameter: $50.17 \pm 0.90 \mathrm{~nm}, \mathrm{~S} . \mathrm{D} .=2.95 \mathrm{~nm} ; n=11 \mathrm{PDs}, \mathrm{N}=3$ for RBE ${ }^{\mathrm{KO} / \mathrm{KO}}$ vs. $47.81 \pm 0.60 \mathrm{~nm}, \mathrm{~S}$. D. $=1.70 ; n=9$ ribbons, $N=3$ for RBE ${ }^{W T / W T} ; p=0.06$, Mann-Whitney-Wilcoxon test, Figure 3-figure supplement 1D, supplementary file 1; RA/RA-SV diameter: $49.71 \pm 0.83 \mathrm{~nm}$, S.D. = 2.75; $n=11$ PDs, $\mathrm{N}=3$ for RBE $\mathrm{KO} / \mathrm{KO}$ vs. $49.80 \pm 0.78 \mathrm{~nm}$, S.D. $=2.35 ; n=9$ ribbons, $\mathrm{N}=3$ for RBE ${ }^{\mathrm{WT} / \mathrm{WT}}$; $\mathrm{p}=0.71$, Mann-Whitney-Wilcoxon test; Figure 3-figure supplement 1G, supplementary file 1). We presume that differences in the comparison of $\mathrm{RBE}^{\mathrm{KO} / \mathrm{KO}}$ and $\mathrm{RBE}{ }^{\mathrm{WT}} / \mathrm{WT}$ between the random section and electron tomography analysis primarily reflects the larger number of $A Z$ analyzed by the former approach.

We then used confocal and stimulated emission depletion (STED) super-resolution immunofluorescence microscopy in order to study the abundance and spatial organization of presynaptic Cav1.3 $\mathrm{Ca}^{2+}$-channels (Neef et al., 2018), which contribute more than $90 \%$ of the voltage-gated $\mathrm{Ca}^{2+}$-influx into IHCs (Platzer et al., 2000; Brandt et al., 2003; Dou et al., 2004). Organs of Corti from 3week-old $\mathrm{RBE}^{\mathrm{KO} / \mathrm{KO}}$ and $\mathrm{RBE}^{\mathrm{WT} / \mathrm{WT}}$ mice were processed in parallel for immunohistochemistry and imaging. Cav1.3 $\mathrm{Ca}^{2+}$-channels remained clustered at $\mathrm{RBE}^{\mathrm{KO} / \mathrm{KO}} \mathrm{AZs}$ and were identified as $\mathrm{Cav} 1.3$ 

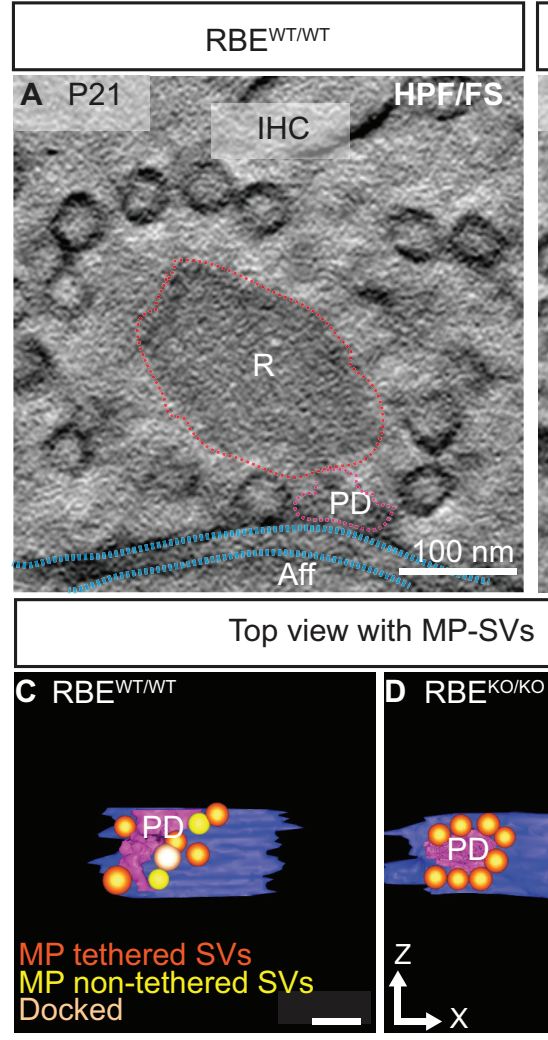

G Tomogram analysis
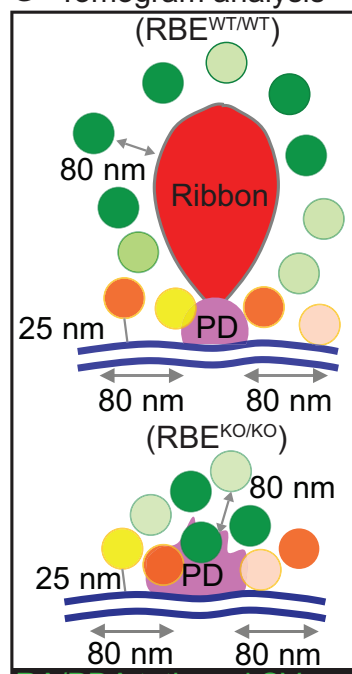

\section{RA/PDA tethered SVs}

RA/PDA non-tethered SVs MP tethered SV

MP non-tethered SVs

\section{Docked}

D RBE ${ }^{\text {WTWT }} N=3$ animals,

$\begin{aligned} n=9 & \text { ribbons } \\ & \end{aligned}$ $n=11$ PDs

$$
\text { , }
$$

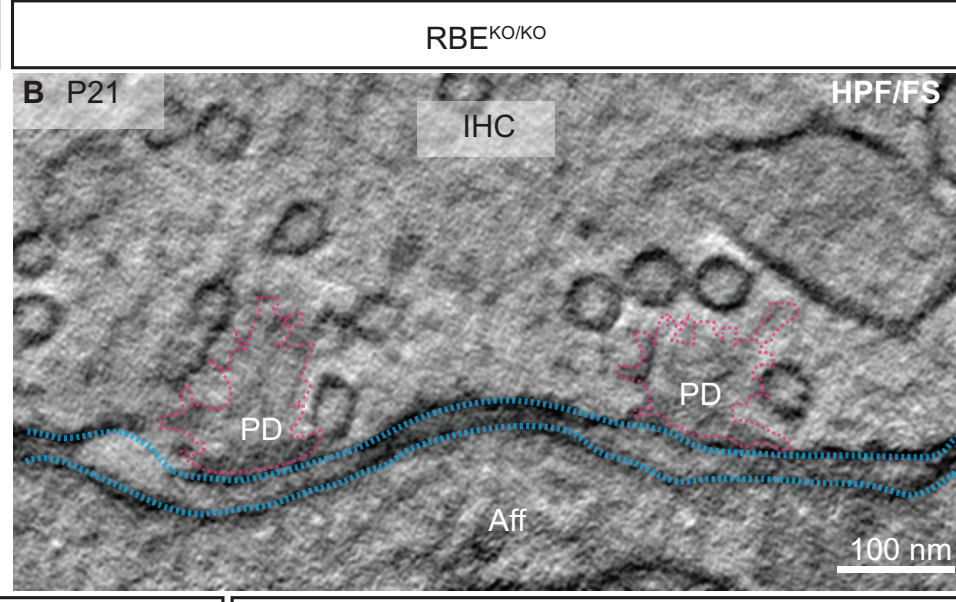

Front view with RA/PDA-SVs
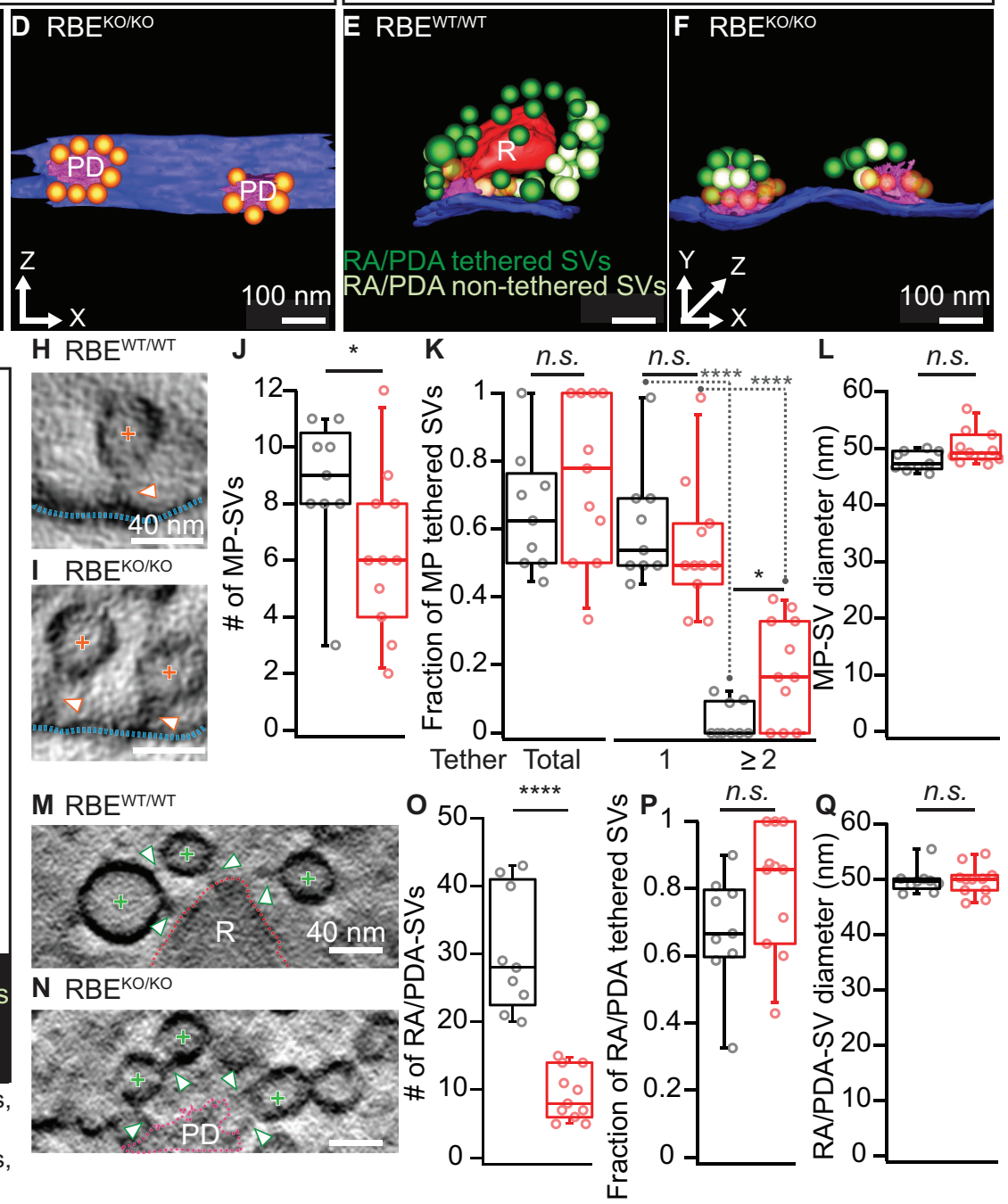

Figure 3. Electron tomography analysis of synaptic ultrastructure obtained after HPF/FS. (A, B) Exemplary virtual electron tomographic sections of P21 $\mathrm{RBE}^{\mathrm{WT} / \mathrm{NT}}(\mathrm{A})$ and $\mathrm{RBE}^{\mathrm{KO} / \mathrm{KO}}$ (B) highlight the ribbon $\mathrm{R}$ in red, the presynaptic density (PD) in magenta and the AZ membrane with blue dotted lines. No synaptic ribbons, but two PDs were observed in $\mathrm{RBE}^{\mathrm{KO} / \mathrm{KO}}$ (B). Scale bars $=100 \mathrm{~nm}$. (C-F) 3D rendered models of RBE ${ }^{\mathrm{WT} / W T}(\mathrm{C}, \mathrm{E})$ and $\mathrm{RBE} \mathrm{KO}^{\mathrm{K} / \mathrm{KO}}(\mathrm{D}, \mathrm{F})$ IHC synapses. (C, D) The top view depicts the MP-SV pool with tethered (orange), non-tethered (yellow) and docked (light orange) SVs. For clarity

Figure 3 continued on next page 
Figure 3 continued

ribbons, RA/PDA-SVs are removed. Scale bars $=100 \mathrm{~nm}$. $(E, F)$ The front view shows the RA/PDA-SV pool from RBE ${ }^{\mathrm{WT} / \mathrm{WT}}(\mathrm{E})$ and RBE ${ }^{\mathrm{KO} / \mathrm{KO}}(\mathrm{F})$ IHCs. Tethered (dark green) and non-tethered (light green) RA/PDA-SVs. For the ease of visualization, the MP-SV pool is transparent here and other synaptic structures such as ribbon (red), PD (magenta) and AZ membrane (blue) are indicated. Scale bars $=100 \mathrm{~nm}$. (G) Illustrations show the tomogram analysis parameters comparable to 2D-random section analysis (Figure 2), in addition to that the vesicle pools are subdivided into tethered, non-tethered and docked SVs. (H, I, M, N) Representative tomogram virtual sections of membrane-tethered MP-SVs (H, I; orange cross), ribbon/PD tethered SVs and ribbon/PD proximal interconnecting SVs $\left(M, N\right.$; green cross) in $\operatorname{RBE}^{W T / W T}(H, M)$ and in $\operatorname{RBE}^{K O / K O}(\mathrm{I}, \mathrm{N})$. Tethers are marked with a white arrowhead and other synaptic entities are color-coded similar to (A, B). Scale bars $=40 \mathrm{~nm}$. (J-L) Quantification for the MP-SV pool is depicted; $n=9$ ribbons, $N=3$ animals for RBE ${ }^{\mathrm{WT} / W T}$ and $n=11 \mathrm{PDs}, \mathrm{N}=3$ animals for RBE ${ }^{\mathrm{KO} / \mathrm{KO}}$. Fewer MP-SV were observed in RBE ${ }^{\mathrm{KO} / \mathrm{KO}}$ ( $\mathrm{J} ; \mathrm{p}=0.04, \mathrm{Mann}-\mathrm{Whitney-Wilcoxon}$ test). The fraction of tethered MP-SVs was unaltered in $\mathrm{RBE}^{\mathrm{KO} / \mathrm{KO}}\left(\mathrm{K} ; \mathrm{p}=0.30\right.$, t-test). Most of the SVs were tethered by a single tether in both RBE ${ }^{\mathrm{KO} / K O}$ and $\mathrm{RBE}^{\mathrm{WT} / \mathrm{WT}}$. Significantly more SVs with multiple-tethers were observed in RBE ${ }^{\mathrm{KO} / K O}(\mathrm{~K}$; single tethered MP-SVs, multiple-tethered MP-SVs: n.s.: $\mathrm{p}>0.05$, : $p=0.01, * \star * *: p<0.0001$, Tukey's test). MP-SV diameter was unaltered in RBE ${ }^{K O} / K O(L ; p=0.06$, Mann-Whitney-Wilcoxon test). (O-Q) Quantification for the RA/PDA-SVs, sample size is same as for the MP-SV analysis. Significantly fewer PDA-SVs were observed in RBE ${ }^{\mathrm{KO} / \mathrm{KO}}\left(\mathrm{O} ; \mathrm{RBE}{ }^{\mathrm{KO} / \mathrm{KO}}: \mathrm{p}<0.0001, \mathrm{Mann}-\right.$ Whitney-Wilcoxon test). The fraction of PDA tethered SVs in RBE ${ }^{K O} / K O$ was comparable to RA tethered SVs in RBE ${ }^{W T / W T}$ (P; $p=0.12, t$-test). SV diameters were unaltered in the RA/PDA vesicle pool ( $Q ; p=0.06$, Mann-Whitney-Wilcoxon test). Box plots show 10, 25, 50, 75 and $90^{\text {th }}$ percentiles with individual data points overlaid. See Figure 3-figure supplement 1 and supplementary file 1 for modified tomogram analysis according to Jung et alı, 2015 a. DOI: https://doi.org/10.7554/eLife.29275.007

The following figure supplement is available for figure 3 :

Figure supplement 1. Electron tomogram analysis according to Jung et al., 2015a. DOI: https://doi.org/10.7554/eLife.29275.008

labeling juxtaposed to PSD-95 immunofluorescent spots (Figure 4A). In order to analyze the spatial organization of synaptic $\mathrm{Ca}^{2+}$-channels, we performed 3-color, 2D-STED immunofluorescence imaging for Cav1.3, bassoon (as a PD-marker), and PSD-95. While more than $80 \%$ of the RBE ${ }^{\text {WT/WT }}$ synapses showed the typical stripe-like co-alignment of Cav1.3 and bassoon immunofluorescence (Neef et al., 2018), imaging of $\mathrm{RBE}^{\mathrm{KO} / \mathrm{KO}}$ synapses indicated a high prevalence (over $70 \%$ ) of smaller, rounder and often several $\mathrm{Ca}^{2+}$-channel clusters and PDs per synaptic contact (Figure 4B, C). We then quantified stripe-like clusters by measuring their long and short axis using 2D Gaussian fits and found no differences between $\mathrm{RBE}^{\mathrm{KO} / \mathrm{KO}}$ and RBE ${ }^{\mathrm{WT}} / \mathrm{WT}$ AZs (Figure 4D). Finally, we quantified the number of $\mathrm{Ca}_{\mathrm{V}}$ 1.3-immunofluorescent structures per contact (as indicated by PSD-95 immunofluorescence). While more than $80 \%$ of RBE ${ }^{\mathrm{WT} / \mathrm{WT}}$ synapses displayed a single cluster, over $60 \%$ of the $\mathrm{RBE}^{\mathrm{KO} / \mathrm{KO}}$ synapses contained two or more $\mathrm{Ca}^{2+}$-channel clusters (Figure 4E). Hence, the average number of Cav1.3-immunofluorescent structures was significantly higher at $\mathrm{RBE}^{\mathrm{KO} / \mathrm{KO}}$ synapses compared to RBE ${ }^{\mathrm{WT} / \mathrm{WT}}(2.06 \pm 0.09$, S.D. $=1.16 ; n=178$ spots, $\mathrm{N}=3$ vs. $1.16 \pm 0.03$, S.D. = 0.38; $n=183$ spots, $N=2 ; p<0.0001$, Mann-Whitney-Wilcoxon test) and we likely underestimated this difference due to the low resolution of 2D-STED in the z-axis. In summary, our results indicate that RIBEYE-disruption transforms the single ribbon-type $A Z$ into a complex presynaptic organization with multiple conventional-like AZs facing the postsynaptic bouton.

\section{Altered operating range of presynaptic $\mathrm{Ca}^{2+}$-influx at ribbonless IHC synapses}

Next, we combined whole-cell patch-clamp with confocal $\mathrm{Ca}^{2+}$-imaging of IHCs to study $\mathrm{Ca}^{2+}$-influx at the whole IHC and single synapse levels using $5 \mathrm{mM}\left[\mathrm{Ca}^{2+}\right]_{e}$ to augment the signal to noise. Using step-depolarizations in conditions that isolated the $\mathrm{Ca}^{2+}$-current (see Materials and methods), we probed the amplitude and voltage-dependence of $\mathrm{IHC} \mathrm{Ca}{ }^{2+}$-influx (Figure 5A). The amplitude of $\mathrm{Ca}^{2+}$-influx (Figure $5 \mathrm{~A}_{i}$; for $\mathrm{Ca}^{2+}$-current density, see Figure $5 \mathrm{~A}_{i i}$ ) was unaltered in $\mathrm{RBE}{ }^{\mathrm{KO} / \mathrm{KO}}$ IHCs ($151 \pm 12.9 \mathrm{pA}$, S.D. $=59 \mathrm{pA} ; n=21 \mathrm{IHCs}, \mathrm{N}=8$ in $\mathrm{RBE}^{\mathrm{KO} / \mathrm{KO}}$ vs. $-161 \pm 15.4 \mathrm{pA}$, S.D. $=71 \mathrm{pA}$; $n=21 \mathrm{IHCs}, \mathrm{N}=9$ in RBE ${ }^{\mathrm{WT} / \mathrm{WT}} ; \mathrm{p}=0.62$, t-test), in agreement with findings in retinal bipolar neurons (Maxeiner et al., 2016) but in contrast to our previous findings in ribbon-deficient IHCs of bassoon mutant mice (Khimich et al., 2005; Frank et al., 2010; Jing et al., 2013). Kinetics of $\mathrm{Ca}^{2+}$-channel activation were unchanged (Figure 5B), whereas inactivation kinetics were slightly faster in the $\mathrm{RBE}^{\mathrm{KO} / \mathrm{KO}} \mathrm{IHCs}$ (smaller residual $\mathrm{Ca}^{2+}$-current at $200 \mathrm{~ms}$ of depolarization (normalized to the peak current): $0.82 \pm 0.007$, S.D. $=0.02 ; n=10 \mathrm{lHCs}, \mathrm{N}=5$ for $\mathrm{RBE}^{\mathrm{KO} / \mathrm{KO}}$ vs. $0.85 \pm 0.01$, S.D. = 0.04; $n=11 \mathrm{lHCs}, \mathrm{N}=7$, in the RBE ${ }^{\mathrm{WT} / \mathrm{WT}}$ condition; $\mathrm{p}=0.017$, Mann-Whitney-Wilcoxon test; Figure 5D). When analyzing the voltage-dependence of $\mathrm{Ca}^{2+}$-channel activation (Figure $5 \mathrm{C}$ ), we found a small (2 

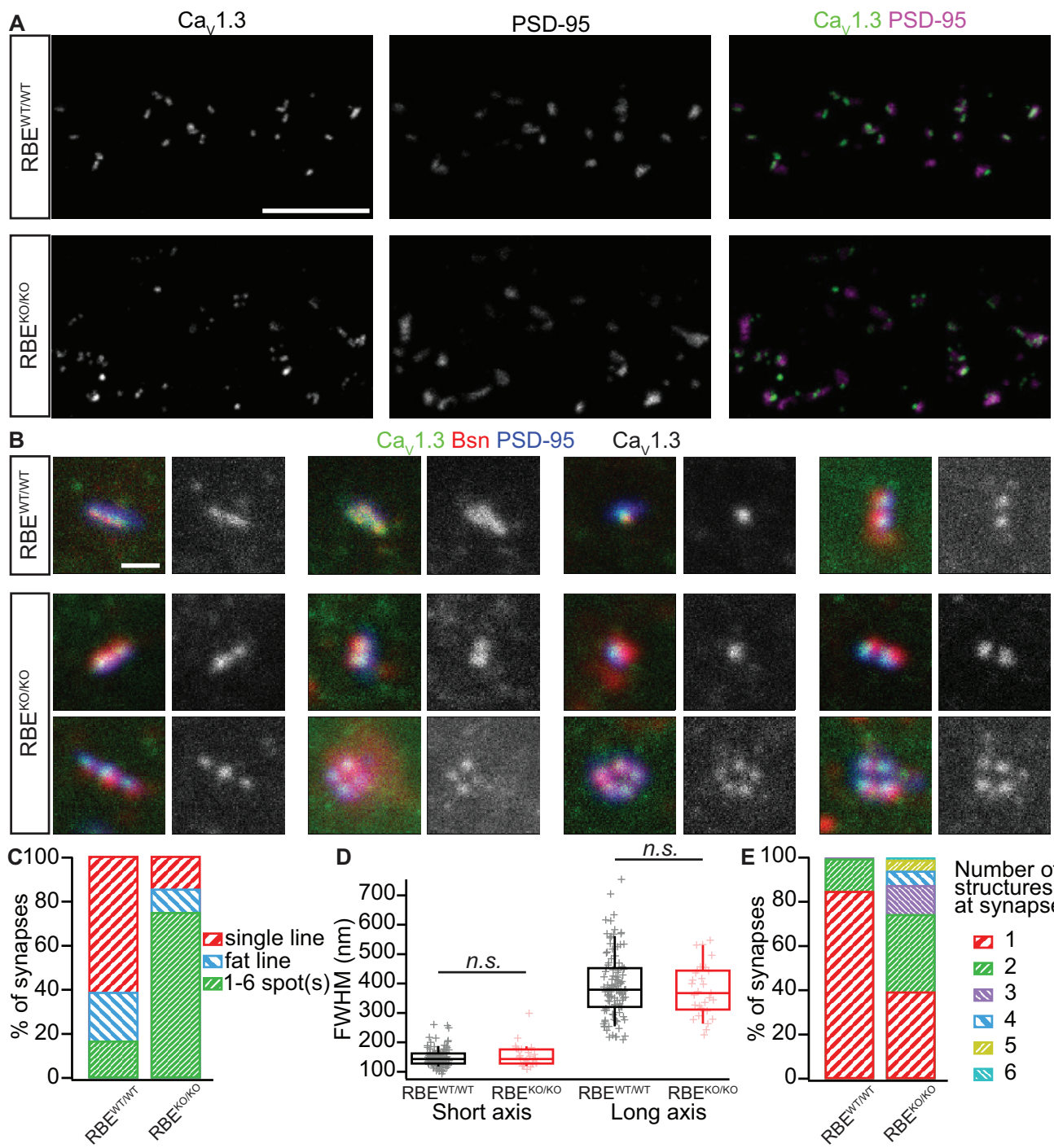

D
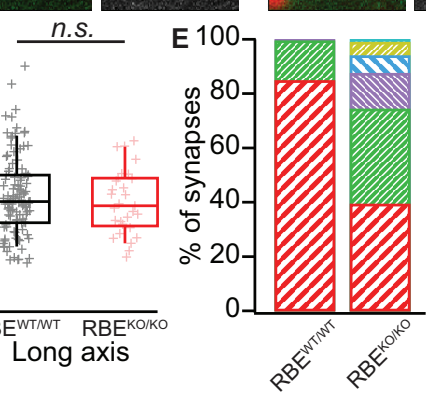

Number of at synapse

[a 1

ए1 2

为 3

$\triangle 4$

ए 5

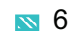

Figure 4. Analyzing AZ $\mathrm{Ca}^{2+}$-channel clusters using confocal and STED immunofluorescence microscopy. (A) Maximal projections of confocal sections from organs of Corti immunolabeled for Cav1.3 $\mathrm{Ca}^{2+}$-channels (left column) and PSD-95 (middle column). The merged pictures (right column) show their juxtaposition in both conditions. While we mostly found one linear/spot-like $\mathrm{Ca}^{2+}$-channel cluster per PSD in RBEWT/WT IHCs (top row), we often observed several spots per PSD in RBE ${ }^{\mathrm{KO} / \mathrm{KO}} \mathrm{HCs}$ (bottom row). Scale bar $=5 \mu \mathrm{m}$. (B) Triple co-labeling of Cav1.3 (green), bassoon (red), and PSD-95 (blue) at several IHC AZs from RBE ${ }^{W T / W T}$ and RBE ${ }^{\mathrm{KO} / K O}$ mice, imaged in 2D-STED (Cav1.3 and PSD-95) and confocal mode (bassoon), showing that Cav1.3 $\mathrm{Ca}^{2+}$-channels cluster at AZs in IHCs of both genotypes. Cav1.3 immunofluorescence is displayed in gray next to the merged image for better visualization. Scale bar $=500 \mathrm{~nm}$. (C) $178 \mathrm{RBE}^{\mathrm{WT} / \mathrm{WT}}$ and $183 \mathrm{RBE}^{\mathrm{KO} / \mathrm{KO}}$ synapses were categorized according to the pattern of Cav1.3

immunofluorescence found by assigning them to a group of either line-shaped clusters, fat line-shaped clusters or one/multiple spots. A markedly higher fraction of synapses was found to display a spot-like Cav1.3-signal in RBE ${ }^{\mathrm{KO} / \mathrm{KO}}$ than in RBE ${ }^{\mathrm{WT} / \mathrm{WT}}$ IHCs. (D) Fitting of a 2D-Gaussian function to the immunofluorescence data of the line-shaped Cav1.3 clusters showed no difference in terms of size between RBE ${ }^{\mathrm{WT} / \mathrm{WT}}$ and RBE ${ }^{\mathrm{KO} / \mathrm{KO}}$ clusters, as estimated by the full width at half maximum of the Gaussian's short and long axis. Box plots show 10, 25, 50, 75 and $90^{\text {th }}$ percentiles with individual data points overlaid. (E) Quantification of the number of fluorescent structures (lines or spots) labeled by the anti-Cav1.3 antibody at RBE ${ }^{\text {WT/WT }}$ and $\mathrm{RBE}^{\mathrm{KO} / \mathrm{KO}}$ synapses showed a significantly increased number in the knockout ( $p<0.0001$, Mann-Whitney-Wilcoxon test).

DOI: https://doi.org/10.7554/eLife.29275.009

$\mathrm{mV}$ ) but significant depolarizing shift of the potential of half-maximal $\mathrm{Ca}^{2+}$-channel activation, $\mathrm{V}_{\mathrm{h}}$ (Figure $5 C_{i},-22.96 \pm 0.43 \mathrm{mV}$, S.D. $=2.39 \mathrm{mV} ; n=21 \mathrm{IHCs}, \mathrm{N}=8$ in $\mathrm{RBE}^{\mathrm{KO} / \mathrm{KO}}$ vs. $-25.04 \pm 0.65$ $\mathrm{mV}, \mathrm{S} . \mathrm{D} .=2.98 \mathrm{mV} ; \mathrm{n}=21 \mathrm{IHCs}, \mathrm{N}=9$ in RBE ${ }^{\mathrm{WT} / \mathrm{WT}} ; \mathrm{p}=0.017$, t-test). When analyzed in a smaller data set recorded in $2 \mathrm{mM}\left[\mathrm{Ca}^{2+}\right]_{\mathrm{e}}$ the depolarized $\mathrm{V}_{\mathrm{h}}$-shift did not reach statistical significance (data 


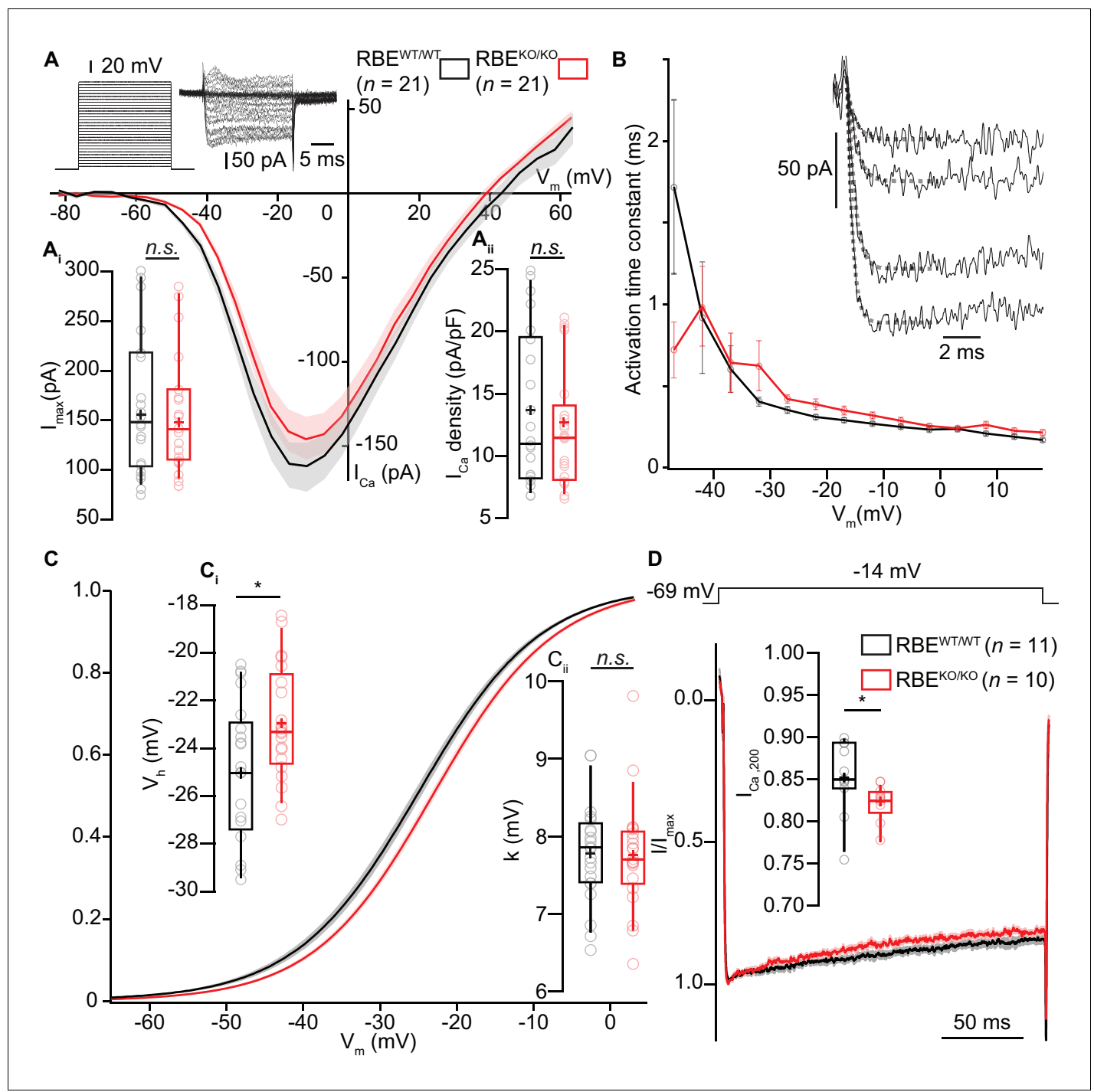

Figure 5. $\mathrm{IHC} \mathrm{Ca}^{2+}$-influx shows normal amplitude but a mild depolarized shift in voltage-dependence. (A) IV-relationship of the whole-cell Ca ${ }^{2+}$ current in RBE ${ }^{\mathrm{WT} / \mathrm{WT}}$ (black, $n=21$ cells, $\mathrm{N}=8$ ) and $\mathrm{RBE}^{\mathrm{KO} / \mathrm{KO}}$ (red, $n=21$ cells, $\mathrm{N}=8$ ) IHCs show comparable (sign-inverted) current amplitudes $\left(\mathrm{A}_{\mathrm{i}}\right.$, $\mathrm{p}=0.62$, t-test) and density $\left(\mathrm{A}_{\mathrm{i} i}, \mathrm{p}=0.37\right.$, t-test.). Mean (line) \pm S.E.M. (shaded areas) are displayed as for $(\mathbf{C}, \mathrm{D})$. The protocol, consisting of $20 \mathrm{~ms}$ steps of $5 \mathrm{mV}$ from -82 to $+63 \mathrm{mV}$, as well as exemplary resulting currents, aredisplayed in the left. Box plots show 10, 25, 50, 75 and 90 th percentiles with individual data points overlaid, means are shown as crosses, as for (C, D). (B) Activation time constants (mean \pm S.E.M.) of Ca ${ }^{2+}$-currents at different potentials were obtained by fitting a power exponential equation to the first $5 \mathrm{~ms}$ of the current traces, revealing no differences between conditions. (C) Fractional activation of the whole-cell $\mathrm{Ca}^{2+}$-current derived from the IV-relationships (A) was fitted to a Boltzmann function. (Ci) Box plots of the voltage for half-maximal activation $V_{h}$ and $V_{h}$-estimates of individual IHCs show a depolarized shift of the fractional activation of the Cav $1.3 \mathrm{Ca}^{2+}$-channels in the $\mathrm{RBE}^{\mathrm{KO} / \mathrm{KO}} \mathrm{IHCs}(\mathrm{p}=0.029$, t-test). (Cii) Box plots of the voltage-sensitivity or slope factor $\mathrm{k}$ and $\mathrm{k}$-estimates of individual IHCs illustrate comparable voltage sensitivity between both conditions ( $p=0.67$, t-test). (D) Average peak-normalized $\mathrm{Ca}^{2+}$-currents resulting from $200 \mathrm{~ms}$ depolarizations to -14 $\mathrm{mV}$. We observe an enhanced inactivation in ribbonless IHCs, quantified as a reduced residual $\mathrm{Ca}^{2+}$-current (inset). ( $n=10 \mathrm{cells}, \mathrm{N}=5 \mathrm{for} \mathrm{RBE}{ }^{\mathrm{KO} / \mathrm{KO}}$ and $n=11$ cells, $N=7$ in the RBE ${ }^{\text {WT }}$ WT; $p=0.017$, Mann-Whitney-Wilcoxon test).

not shown). The average voltage-sensitivity of activation (slope factor $\mathrm{k}$ ) was not altered (5 $\mathrm{mM}$ $\left[\mathrm{Ca}^{2+}\right]_{\mathrm{e}}: \mathrm{p}=0.67$, t-test, Figure $\left.5 \mathrm{C}_{\mathrm{ii}}\right)$. Together, this suggests a RIBEYE/ribbons-mediated regulation of $\mathrm{IHC} \mathrm{Ca}{ }^{2+}$-channels affecting their voltage-range of operation as well as their inactivation kinetics.

We then used the low-affinity $\mathrm{Ca}^{2+}$-indicator dye Fluo-4FF $(800 \mu \mathrm{M})$ to study $\mathrm{Ca}^{2+}$-influx at individual IHC AZs (Frank et al., 2009) using a spinning-disk confocal microscope that allows rapid registering and recording of the majority of the IHC synapses (Figure 6A, Ohn et al., 2016). We chose conditions in which the $\mathrm{Ca}^{2+}$-indicator fluorescence approximates synaptic $\mathrm{Ca}^{2+}$-influx (Frank et al., 
A
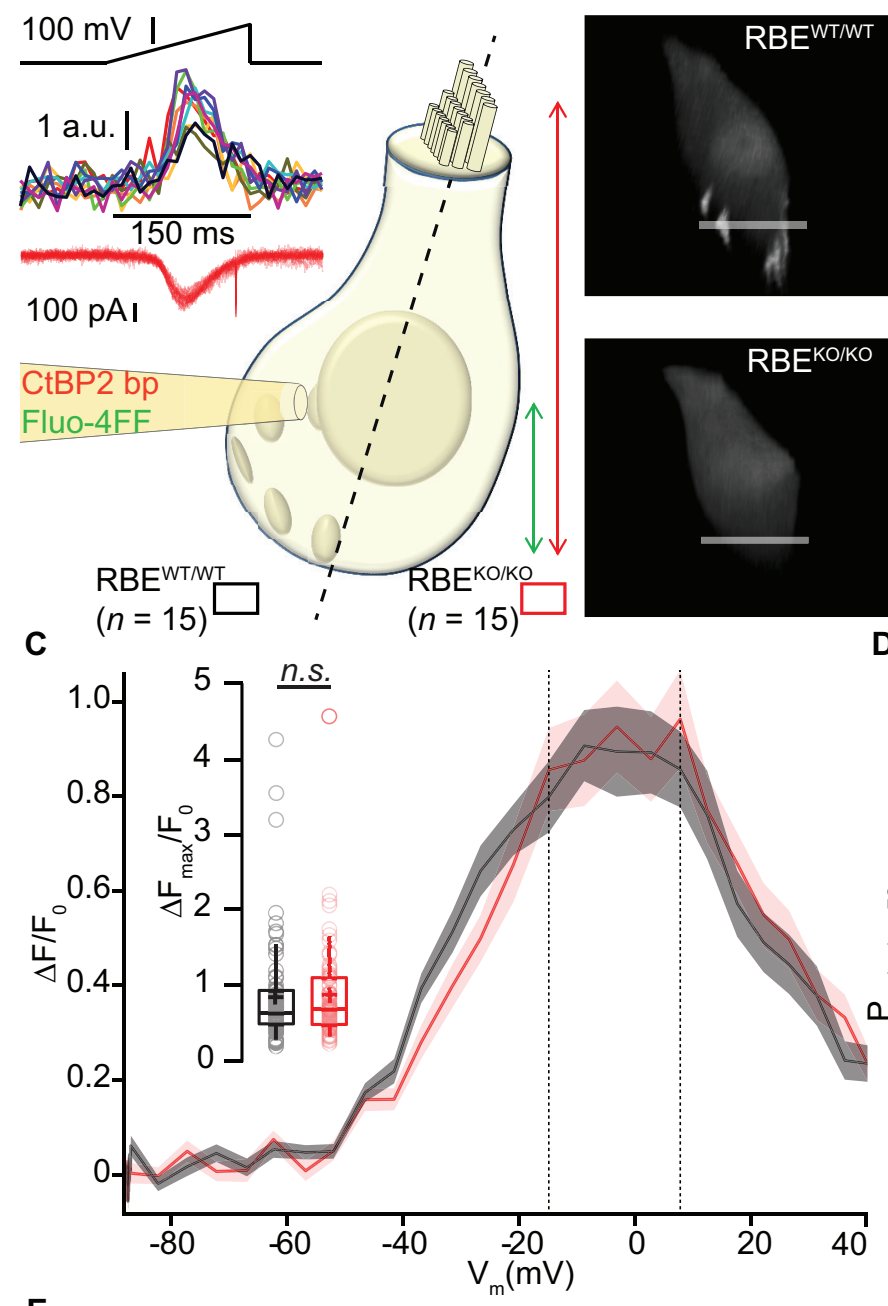

E

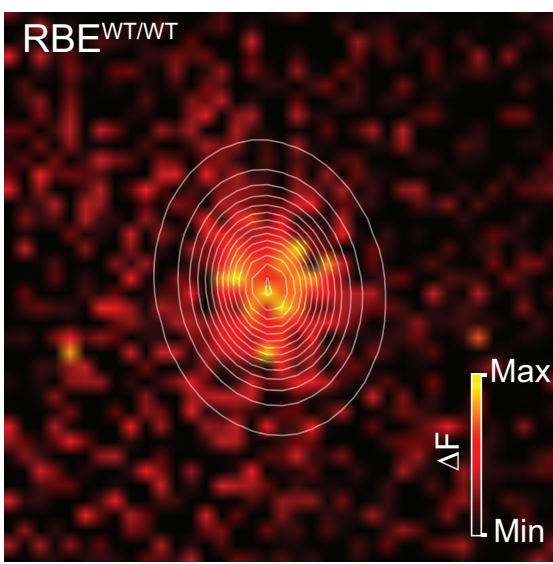

B
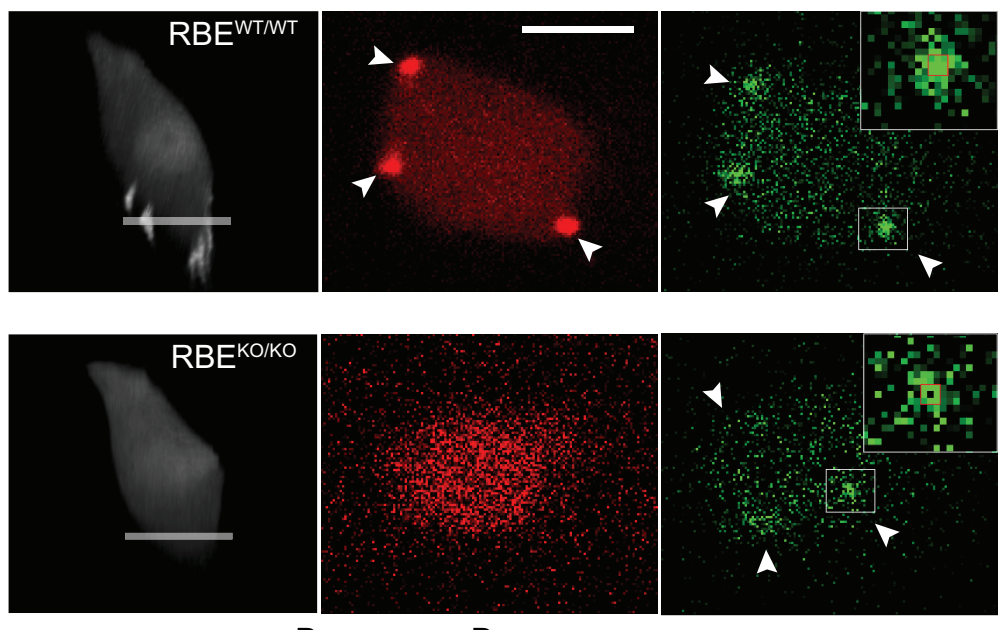

D 1
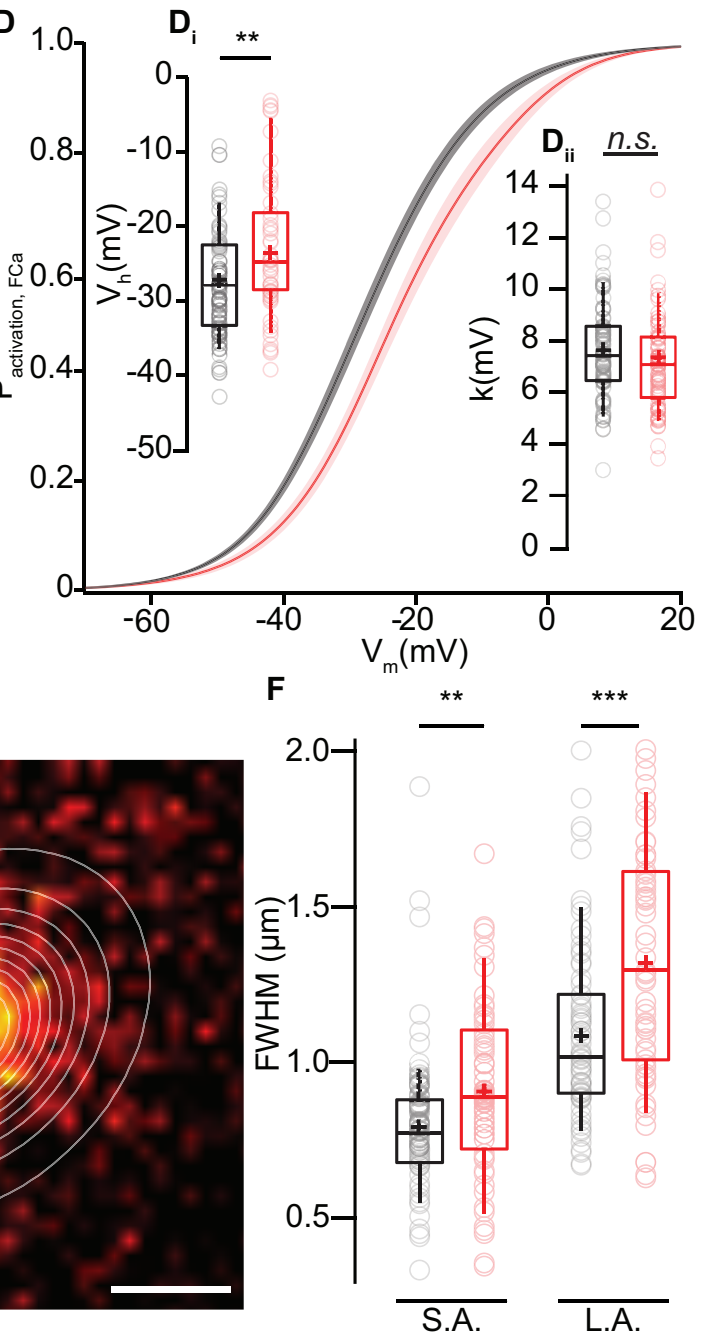

Figure 6. Synaptic $\mathrm{Ca}^{2+}$-influx shows normal amplitude but shifted voltage-dependence and broader spread. (A, B) IHCs were patch-clamped at the modiolar basolateral face, loaded with TAMRA-CtBP2-binding peptide and the low affinity $\mathrm{Ca}^{2+}$-indicator Fluo-4FF, and scanned in the red channel after loading for 4 min to image TAMRA-labeled ribbons, nuclei, and cytosol. 3D projection of TAMRA fluorescence shows the absence of ribbons in $\mathrm{RBE}^{\mathrm{KO} / \mathrm{KO}} \mathrm{IHCs}$ (B: $3 \mathrm{D}$ projection and red channel). Voltage-ramps from -87 to $+63 \mathrm{mV}$ during $150 \mathrm{~ms}$ ( $\mathrm{A}$ : left top) were used to trigger

Figure 6 continued on next page 
Figure 6 continued

synaptic hotspots of Fluo-4FF fluorescence (A: left middle, $10 \mathrm{AZs}$ in one exemplary RBE ${ }^{\mathrm{WT} / W T}$ IHC, $\mathrm{B}$ : green channel, marked by arrowheads; $\Delta \mathrm{F}$ :

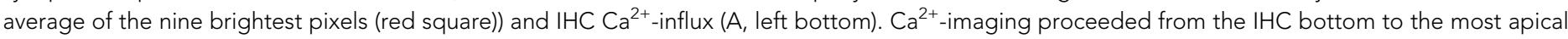
ribbon in RBE ${ }^{\mathrm{WT}} / \mathrm{WT}$, and from IHC bottom to $+12 \mu \mathrm{m}$ (typically reaching the bottom of nucleus) in $\mathrm{RBE}^{\mathrm{KO} / \mathrm{KO}}$. Scale bar $=5 \mu \mathrm{m}$. (C) FV-relationship ( $\Delta \mathrm{F} /$ $F_{0}$ vs. depolarization level in ramp, protocol as in A): approximating the voltage-dependence of synaptic Ca ${ }^{2+}$-influx.Mean (line) \pm S.E.M. (shaded areas) are displayed as for (D). (Ci) $\Delta \mathrm{F}_{\text {max }} / \mathrm{F}_{0}$ was calculated by averaging 5 values at the FV-peak (between the dotted lines) and was comparable between $\mathrm{RBE}^{\mathrm{WT} / \mathrm{WT}}\left(\mathrm{n}=78 \mathrm{AZs}\right.$ for 15 cells, $\mathrm{N}=8$ ) and RBE ${ }^{\mathrm{KO} / \mathrm{KO}} \mathrm{IHCs}(n=61 \mathrm{AZs}$ for 15 cells, $N=7$ ) ( $p=0.20$, Mann-Whitney-Wilcoxon test). Box plots show 10, $25,50,75$ and $90^{\text {th }}$ percentiles with individual data points overlaid, means are shown as crosses, as for (D, F). (D) Fractional activation curves derived from fits to the FV-relationships (C) were fitted to a Boltzmann function. Mean (line) \pm S.E.M. (shaded areas) are displayed. (Di) The voltage for halfmaximal activation $V_{h}$ was significantly different between RBE ${ }^{\mathrm{WT} / \mathrm{WT}}$ ( $n=68 \mathrm{AZs}$ for $15 \mathrm{IHCs}, \mathrm{N}=8$ ) and RBE ${ }^{\mathrm{KO} / \mathrm{KO}}$ ( $n=55 \mathrm{AZs}$ for $15 \mathrm{lHCs}, \mathrm{N}=7$ ) $\mathrm{AZs}$ $\left(p=0.0029\right.$, t-test), while the voltage-sensitivity or slope factor $k$ (Dii) not ( $p=0.42$, t-test). (E) Exemplary $\Delta F$ pictures of Fluo- $4 F F$ hotspots at RBE ${ }^{W T / W T}$ (left) and RBE ${ }^{K O} / K O$ (right) synapses fitted and overlaid by 2D-Gaussian functions to estimate spatial extent as full width at half maximum (FWHM) for the short axis (S.A.) and the long axis (L.A.). Scale bar $=1 \mu \mathrm{m}$. (F) Ribbonless synapses of RBE ${ }^{\mathrm{KO} / \mathrm{KO}}$ IHCs showed a greater spatial spread of the Fluo4FF fluorescence change. FWHM calculated from the Gaussian fitting to the Fluo-4FF fluorescence hotspot was larger for both axes in RBE ${ }^{\mathrm{KO} / \mathrm{KO}}(\mathrm{n}=61$ AZs for $15 \mathrm{IHCs}, \mathrm{N}=8$ ) compared to RBE ${ }^{W T / W T}$ ( $n=74$ AZs for $15 \mathrm{IHCs}, \mathrm{N}=7$ ) (L.A.: $p=0.00016$; S.A.: $p=0.0029$, t-test).

DOl: https://doi.org/10.7554/eLife.29275.011

The following figure supplement is available for figure 6:

Figure supplement 1. Semi-quantitative immunofluorescence analysis of the three main proteinaceous $\mathrm{Ca}^{2+}$-buffers. DOI: https://doi.org/10.7554/eLife.29275.012

2009; Ohn et al., 2016) and henceforth refer to synaptic $\mathrm{Ca}^{2+}$-influx when describing observations based on hotspots of $\mathrm{Ca}^{2+}$-indicator fluorescence at the basolateral IHC membrane. Prior to analysis of synaptic $\mathrm{Ca}^{2+}$-influx, we imaged fluorescently-conjugated CtBP2-binding peptide (Zenisek et al., 2004), which bound to the ribbon-occupied AZs in RBE ${ }^{\text {WT/WT }}$ IHCs while it only caused nuclear and diffuse cytosolic fluorescence in the ribbonless RBE ${ }^{\mathrm{KO} / K O} \mathrm{IHCs}$ (Figure 6B). We then employed ramp-depolarizations to assess amplitude and voltage-dependence of $\mathrm{Ca}^{2+}$-influx at the synapses located in the subnuclear, basal part of the IHCs (Figure 6A). We found comparable maximal amplitudes of the baseline-normalized fluorescence change $\left(\Delta F / F_{0}, 0.88 \pm 0.08, S . D .=0.66 ; n=61 \mathrm{AZs}\right.$ in $15 \mathrm{IHCs}, \mathrm{N}=7$ for RBE ${ }^{\mathrm{KO} / \mathrm{KO}}$ vs. $0.85 \pm 0.08$, S.D. $=0.68 ; n=78 \mathrm{AZs}$ in $15 \mathrm{lHCs}, \mathrm{N}=8$ for RBE ${ }^{\mathrm{WT} / \mathrm{WT} \text {; }}$ $\mathrm{p}=0.20$, Mann-Whitney-Wilcoxon test; Figure $6 \mathrm{C}$ ). This is compatible with an unaltered number of synaptic $\mathrm{Ca}^{2+}$-channels at $\mathrm{AZs}$ of $\mathrm{RBE}^{\mathrm{KO} / \mathrm{KO}} \mathrm{IHCs}$ and consistent with our observations of normal whole-cell $\mathrm{Ca}^{2+}$-current amplitudes. As previously reported (Frank et al., 2009; Ohn et al., 2016), there was a substantial variation of the maximal $\Delta F / F_{0}$ among the $A Z s$, which was also comparable between $A Z s$ of both genotypes (c.v. $=0.75$ for $\mathrm{RBE}^{\mathrm{KO} / \mathrm{KO}}$ vs. c.v. $=0.80$ for $\mathrm{RBE}{ }^{\mathrm{WT} / \mathrm{WT}}$ ).

Next, we analyzed the voltage-dependence of activation for the synaptic $\mathrm{Ca}^{2+}$-influx as previously described (Ohn et al., 2016). Analysis of fractional activation revealed a depolarized shift in $V_{h}$ by on average $5 \mathrm{mV}$ in $\mathrm{RBE}^{\mathrm{KO} / \mathrm{KO}} \mathrm{IHCs}(-22.76 \pm 1.25 \mathrm{mV}$, S.D. $=9.26 \mathrm{mV} ; n=55 \mathrm{AZs}$ in $15 \mathrm{IHCs}, \mathrm{N}=7$ for $\mathrm{RBE}^{\mathrm{KO} / \mathrm{KO}}$ vs. $-27.37 \pm 0.90 \mathrm{mV}$, S.D. $=7.48 \mathrm{mV} ; \mathrm{n}=68 \mathrm{AZs}$ in $15 \mathrm{IHCs}, \mathrm{N}=8$ for RBE WT/WT; $\mathrm{p}=0.0029$, t-test; Figure $6 \mathrm{D}, \mathrm{Di})$, while the slope factor of voltage-dependent activation was unaltered $\left(p=0.42\right.$, t-test, Figure $\left.6 D_{i i}\right)$. Such a shift in the operating range of synaptic $\mathrm{Ca}^{2+}$-influx is expected to alter spontaneous and sound-evoked transmitter release (see below and Ohn et al., 2016). Finally, we studied the spatial extent of the synaptic $\mathrm{Ca}^{2+}$-signals and estimated Full Width Half Maximum (FWHM) by fitting 2D Gaussian functions to the hotspots of $\mathrm{Ca}^{2+}$-indicator fluorescence and found a greater spread of $\mathrm{Ca}^{2+}$-signals at $\mathrm{RBE}^{\mathrm{KO} / \mathrm{KO}}$ AZs (Figure 6E, F; long axis (L. A.) $=1317 \pm 49 \mathrm{~nm}$, S.D. $=384 \mathrm{~nm}$, short axis (S.A.) $=906 \pm 36 \mathrm{~nm}$, S.D. $=284 \mathrm{~nm} ; n=61$ AZs in 15 IHCs, $N=7$ vs. L.A. $=1083 \pm 33 \mathrm{~nm}$, S.D. $=283 \mathrm{~nm} ;(p=0.00016$, t-test), S.A. $=793 \pm 27 \mathrm{~nm}, \mathrm{~S}$. D. $=233 \mathrm{~nm},\left(p=0.0029\right.$, t-test); $n=74 \mathrm{AZs}$ in $15 \mathrm{lHCs}, \mathrm{N}=8$ for RBE $\left.{ }^{\mathrm{WT} / \mathrm{WT}}\right)$. This larger spread of the presynaptic $\mathrm{Ca}^{2+}$-signals is in agreement with the presence of several Cav1.3-immunofluorescent clusters at $\mathrm{RBE}^{\mathrm{KO} / \mathrm{KO}}$ synapses. In order to exclude lower $\mathrm{IHC} \mathrm{Ca}{ }^{2+}$-buffering to contribute to the observed larger spread of presynaptic $\mathrm{Ca}^{2+}$-signals, we performed semi-quantitative immunofluorescence analysis for the three major cytosolic $\mathrm{Ca}^{2+}$-buffers, the EF-hand $\mathrm{Ca}^{2+}$-binding proteins parvalbumin- $\alpha$, calretinin and calbindin-28k (Pangršič et al., 2015). We did not find any significant differences in their immunofluorescence intensity between IHCs of both genotypes (in arbitrary units, parvalbumin intensity: $2.24 \pm 0.15$, S.D. $=1.04$ for $\mathrm{RBE}^{\mathrm{KO} / \mathrm{KO}}$ vs. $1.88 \pm 0.15, \mathrm{~S} . \mathrm{D}=1.01$ for $\mathrm{RBE}^{\mathrm{WT} /}$ WT,$p=0.08$; calbindin intensity: $0.82 \pm 0.06$, S.D. $=0.43$ for $\mathrm{RBE}^{\mathrm{KO} / \mathrm{KO}}$ vs. $0.95 \pm 0.07$, S.D. $=0.49$ for 
$\mathrm{RBE}^{\mathrm{WT} / \mathrm{WT}}, \mathrm{p}=0.23$; calretinin intensity: $0.91 \pm 0.04$, S.D. $=0.26$ for $\mathrm{RBE}^{\mathrm{KO} / \mathrm{KO}}$ vs. $0.82 \pm 0.04, \mathrm{~S}$. D. $=0.28$ for RBE ${ }^{\mathrm{WT} / \mathrm{WT}}, \mathrm{p}=0.09 ; n=49$ cells and $\mathrm{N}=4$ for both conditions, Mann-Whitney-Wilcoxon test for all; Figure 6-figure supplement 1).

\section{IHC exocytosis is normal for strong depolarizations but mildly reduced for weak ones}

The ribbon has been proposed to play a crucial role in the exocytosis of SVs at the IHC AZ (Khimich et al., 2005). Therefore, we monitored stimulated exocytosis of SVs with perforated-patch whole-cell recordings of exocytic membrane capacitance changes $\left(\Delta C_{m}\right)$. Using IHCs from 2/3-weekold $\mathrm{RBE}^{\mathrm{WT} / \mathrm{WT}}$ and $\mathrm{RBE}^{\mathrm{KO} / \mathrm{KO}}$ mice, we found that $\Delta \mathrm{C}_{\mathrm{m}}$ in response to step-depolarizations to the potential that elicits maximal $\mathrm{Ca}^{2+}$-influx $(-14 \mathrm{mV})$ were not different between $\mathrm{IHCs}$ with or without ribbons. Both, fast exocytosis elicited by depolarizations of up to $20 \mathrm{~ms}$, attributed to the fusion of the readily releasable pool of SVs (RRP, Moser and Beutner, 2000), and longer stimuli, thought to reflect sustained exocytosis, ongoing SV replenishment and fusion, were unaltered in $\mathrm{RBE}{ }^{\mathrm{KO} / \mathrm{KO}} \mathrm{IHCs}$

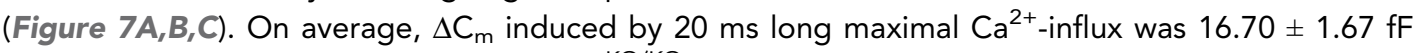
(S.D. $=5.80 \mathrm{fF} ; n=12$ cells, $\mathrm{N}=7$ ) for $\mathrm{RBE}^{\mathrm{KO} / \mathrm{KO}}$ compared to $15.22 \pm 0.98 \mathrm{fF}$ (S.D. $=3.26 \mathrm{fF} ; n=11$ cells, $\mathrm{N}=8$ ) for RBE ${ }^{\mathrm{WT} / W T}$. Exocytic $\Delta \mathrm{C}_{\mathrm{m}}$ elicited by $200 \mathrm{~ms}$ long maximal $\mathrm{Ca}^{2+}$-influx (same $\mathrm{IHCs}$ as for $20 \mathrm{~ms}$ ), on average, amounted to $62.09 \pm 5.40 \mathrm{fF}$ (S.D. $=18.70 \mathrm{fF}$ ) for $\mathrm{RBE}^{\mathrm{KO} / \mathrm{KO}}$ versus $63.28 \pm 6.64 \mathrm{fF}$ (S.D. $=22.04 \mathrm{fF})$ for RBE ${ }^{\mathrm{WT} / \mathrm{WT}}$.

Moreover, trains of 20 step-depolarizations to $-17 \mathrm{mV}$ of $20 \mathrm{~ms}$ pulse duration did not reveal impaired exocytosis in $\mathrm{RBE}^{\mathrm{KO} / \mathrm{KO}} \mid \mathrm{HC}$, even when the inter-stimulus interval time was as short as 160 ms (Figure 7D; $n=11$ cells, $N=5$ for RBE ${ }^{\mathrm{WT} / \mathrm{WT}}$ and $n=13$ cells, $\mathrm{N}=8$ for $\mathrm{RBE}^{\mathrm{KO} / \mathrm{KO}}$ ). We further explored RRP recovery from partial depletion using a paired-pulse protocol (two strong $20 \mathrm{~ms}$ depolarizations to $-14 \mathrm{mV}$ separated by $50,110,260$ and $510 \mathrm{~ms}$ inter-pulse intervals; Figure 7E,F). RRP recovery, estimated as the $\Delta C_{m}$ ratio of the second and the first pulse, was not altered in $\mathrm{RBE}^{\mathrm{KO} / \mathrm{KO}}$ $\mathrm{IHCs}$ at least when probing RRP exocytosis with maximal $\mathrm{Ca}^{2+}$-influx from a hyperpolarized resting potential (Figure 7F). These data are in strong contrast to our previous findings in IHCs of bassoon mutant mice, which we had equivalently analyzed. There, the loss of synaptic ribbons, combined with a loss of functional bassoon resulted in profound deficits in exocytosis (Khimich et alo, 2005; Frank et al., 2010; Jing et al., 2013).

Given the finding of a small depolarized shift in the operating range of $\mathrm{Ca}^{2+}$-channels in $\mathrm{RBE}^{\mathrm{KO} /}$ KO IHCs (Figure 6D), we also probed the voltage-dependence of $\Delta C_{m}$ elicited by $100 \mathrm{~ms}$ step-depolarizations (Figure $7 \mathrm{G}, \mathrm{H}$ ). In agreement with the results obtained at maximal $\mathrm{Ca}^{2+}{ }_{\text {-influx, we did not }}$ find significant differences in $\Delta \mathrm{C}_{\mathrm{m}}$ for stronger depolarizations (e.g. pulses to $-39 \mathrm{mV}$ elicited an average $\Delta \mathrm{C}_{\mathrm{m}}$ of $20.67 \pm 7.46 \mathrm{fF}, \mathrm{S} . \mathrm{D} .=23.58 \mathrm{fF}, n_{\min }=10 \mathrm{IHCs}, \mathrm{N}=9$ for RBE $\mathrm{KO}^{\mathrm{KOO}}$ vs. $24.12 \pm 4.04$ $\mathrm{fF}, \mathrm{S} . \mathrm{D} .=13.98 \mathrm{fF}, \mathrm{n}_{\min }=10 \mathrm{lHCs}, \mathrm{N}=9$ for RBE ${ }^{\mathrm{WT} / \mathrm{WT}} ; \mathrm{p}=0.20$; Mann-Whitney-Wilcoxon test). However, for weaker depolarizations in the range of physiological receptor potentials (Russell and Sellick, 1983), we observed a subtle but significant reduction in exocytosis for RBE ${ }^{\mathrm{KO} / K O} \mathrm{IHCs}$ (Figure $7 \mathrm{H}, \mathrm{p}=0.0115, \mathrm{p}=0.0295$ and $\mathrm{p}=0.1321$ for $-45,-43$ and $-41 \mathrm{mV}$; without definitive outliers as determined by Graphpad Prism: $p=0.0017, p=0.0042$ and $p=0.0489$, respectively; Mann-WhitneyWilcoxon test for all). For instance, depolarization to $-45 \mathrm{mV}$ elicited a $\Delta \mathrm{C}_{\mathrm{m}}$ of $4.79 \pm 2.26 \mathrm{fF}$ for $\mathrm{RBE}^{\mathrm{KO} / \mathrm{KO}}$ (S.D. $=7.14 \mathrm{fF} ; \mathrm{n}_{\min }=10$ cells, $\mathrm{N}=9$ ) compared to $9.85 \pm 1.60 \mathrm{fF}$ for $\mathrm{RBE}^{\mathrm{WT} / \mathrm{WT}}$ (S. D. $=5.05 \mathrm{fF} ; n_{\min }=10$ cells, $\left.\mathrm{N}=8\right)$. The $\mathrm{Ca}^{2+}$-current integral $\left(\mathrm{Ca}^{2+}\right.$-charge, $\left.\mathrm{Q}_{\mathrm{Ca}}\right)$, as well, tended to be reduced for $\mathrm{RBE}^{\mathrm{KO} / \mathrm{KO}} \mathrm{IHC}$ at these mild depolarizations, which, however, did not reach statistical significance (e.g. $\mathrm{Q}_{\mathrm{Ca}}$ for $-45 \mathrm{mV}: 3.90 \pm 0.49 \mathrm{pC}$, S.D. $=1.54 \mathrm{pC}$ for $\mathrm{RBE}^{\mathrm{KO} / \mathrm{KO}}$ vs. $5.15 \pm 0.54 \mathrm{pC}$, S.D. $=1.72 \mathrm{pC}$ for RBE ${ }^{\mathrm{WT} / W T} ; \mathrm{p}=0.1053$; t-test). In summary, we found exocytosis to be unaltered for strong depolarizations but mildly decreased for more physiological stimuli in $\mathrm{RBE}^{\mathrm{KO} / \mathrm{KO}} \mathrm{IHCs}$, which is in line with the findings of the companion paper by Becker et al..

\section{Lack of synaptic ribbons impairs synchronous activation of the auditory pathway}

Next, we studied sound encoding in $\mathrm{RBE}^{\mathrm{KO} / \mathrm{KO}}$ mice in vivo. First, we recorded auditory brainstem responses $(A B R)$ and found a significant reduction in the amplitude of wave I that reflects the SGN compound action potential $\left(1.14 \pm 0.13 \mu \mathrm{V}\right.$, S.D. $=0.38 \mu \mathrm{V}, \mathrm{N}=10$ for $\mathrm{RBE}^{\mathrm{KO} / \mathrm{KO}}$ vs. $3.30 \pm 0.51 \mu \mathrm{V}$, S.D. $=1.54 \mu \mathrm{V}, \mathrm{N}=10$ for RBE ${ }^{\mathrm{WT} / \mathrm{WT}}, \mathrm{p}=0.0007$, NPMC test). This indicates less synchronous $\mathrm{SGN}$ 


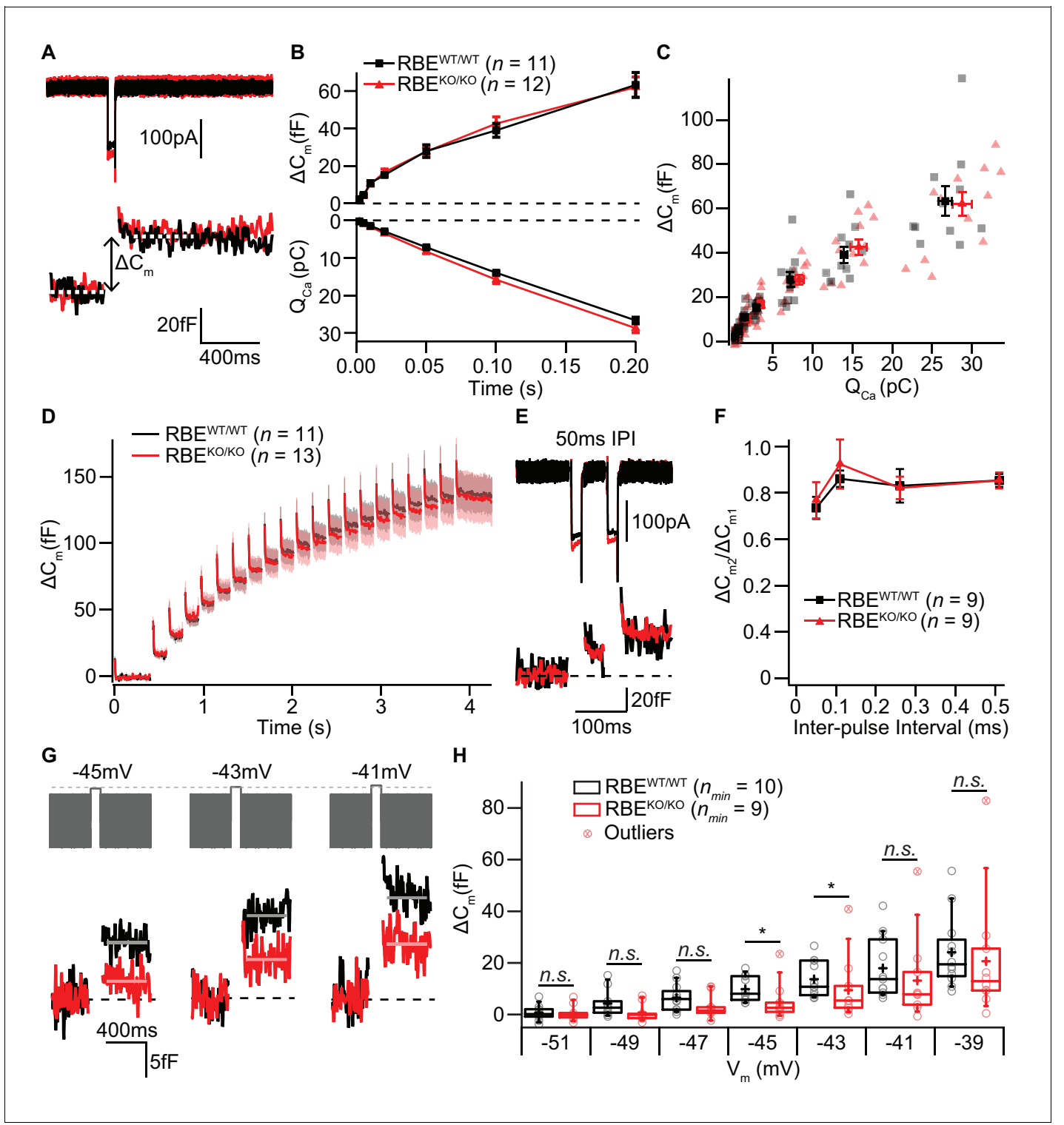

Figure 7. $\mathrm{HC}$ exocytosis is normal for strong depolarizations but mildly reduced for weak ones. (A) Representative $\mathrm{Ca}^{2+}$-currents (top) and corresponding low passed-filtered membrane capacitance $\left(\Delta \mathrm{C}_{m}\right)$ traces recorded from RBE ${ }^{\mathrm{WT} / W T}$ and RBE ${ }^{\mathrm{KO} / \mathrm{KO}}$ IHCs upon $50 \mathrm{~ms}$ depolarizations from -69 to $-14 \mathrm{mV}$. (B) Cumulative exocytosis (exocytic $\Delta \mathrm{C}_{\mathrm{m}}$, top) and corresponding $\mathrm{Ca}^{2+}$-charge $\left(\mathrm{Q}_{\mathrm{Ca}}\right.$, bottom) of RBE ${ }^{\mathrm{WT} / \mathrm{WT}}$ ( $n=11 \mathrm{cells}, \mathrm{N}=8$ ) and $\mathrm{RBE}^{\mathrm{KO} / \mathrm{KO}}$ ( $n=12$ cells, $\mathrm{N}=7$ ) IHCs as a function of stimulus duration (2 to $200 \mathrm{~ms}$ to $-14 \mathrm{mV}$ ) were unaltered in RBE ${ }^{\mathrm{KO} / \mathrm{KO}} \mathrm{IHCs}$. Data is presented as mean \pm S.E.M as for $(F)$. (C) Relating $\Delta C_{m}$ to the corresponding $Q_{C a}$ indicated comparable $\mathrm{Ca}^{2+}$ efficiency of exocytosis between RBE ${ }^{\text {WT/WT }}$ and RBE ${ }^{\text {KO/ }}$ $\mathrm{KO}$ IHCs. Mean \pm S.E.M. for each pulse duration is presented in black and red; individual IHCs data points are overlaid. (D) Mean $\Delta C_{m}$ traces (shaded areas: S.E.M.) in response to trains of $20 \mathrm{~ms}$ depolarizations from -87 to $-17 \mathrm{mV}$ (20 stimuli separated by $160 \mathrm{~ms})$ of RBE ${ }^{\mathrm{WT} / \mathrm{WT}}$ ( $n=11 \mathrm{cells}$, $\mathrm{N}=5$ ) and $\mathrm{RBE}^{\mathrm{KO} / \mathrm{KO}}\left(n=13\right.$ cells, $\mathrm{N}=8$ ) IHCs show comparable exocytic $\Delta \mathrm{C}_{\mathrm{m}}$. (E) Representative low pass-filtered $\Delta \mathrm{C}_{\mathrm{m}}$ traces in response to a pair of $20 \mathrm{~ms}$ pulses to $-17 \mathrm{mV}$, separated by a $50 \mathrm{~ms}$ of inter-pulse interval (IPI). (F) Ratios of exocytosis $\left(\Delta \mathrm{C}_{\mathrm{m} 2} / \Delta \mathrm{C}_{\mathrm{m} 1}\right)$ to a pair of $20 \mathrm{~ms}$ pulses with varying interpulse intervals $\left(50,110,260\right.$ and $510 \mathrm{~ms}$ ) reveal a comparable recovery from RRP depletion between $\mathrm{RBE}^{\mathrm{WT} / \mathrm{WT}}(n=9 \mathrm{cells}, \mathrm{N}=6)$ and RBE ${ }^{\mathrm{KO} / \mathrm{KO}}$ IHCs ( $n=9$ cells, $N=6$ ). (G) Representative low pass-filtered $\Delta C_{m}$ traces in response to 100 ms step-depolarizations to $-45,-43$ and $-41 \mathrm{mV}$. (H) Box plot and single values of $\Delta \mathrm{C}_{m}$ elicited by $100 \mathrm{~ms}$ step-depolarizations of RBEWT/WT $\left(n_{\min }=10\right.$ cells, $\mathrm{N}=8$ ) and RBE ${ }^{\mathrm{KO} / \mathrm{KO}}\left(n_{\min }=9\right.$ cells, $\left.\mathrm{N}=9\right) \mathrm{IHCs}$ to different potentials. Exocytic $\Delta C_{m}$ of $R B E^{K O} / K O$ IHCs was reduced for mild depolarizations $(-45,-43$ and $-41 \mathrm{mV} ; p=0.0115, p=0.0295$ and $p=0.1321$, respectively; $p=0.0017, p=0.0042$ and $p=0.0489$, without definitive outliers; Mann-Whitney-Wilcoxon test), but comparable to RBEWT/WT IHCs at stronger depolarizations ( $-39 \mathrm{mV} ; \mathrm{p}=0.2030$, Mann-Whitney-Wilcoxon test). Box plots show 10, 25, 50, 75 and $90^{\text {th }}$ percentiles with the individual data points overlaid.

DOl: https://doi.org/10.7554/eLife.29275.013 
activation in the absence of synaptic ribbons (Figure $8 A, B$ ). The subsequent ABR waves (Figure 8figure supplement 1) were normal in amplitude (waves II, IV and V, while wave III was reduced) indicating a degree of central auditory compensation for the sound encoding deficit, for example via coincidence detection of converging SGN input in the cochlear nucleus (Joris et alo, 1994;

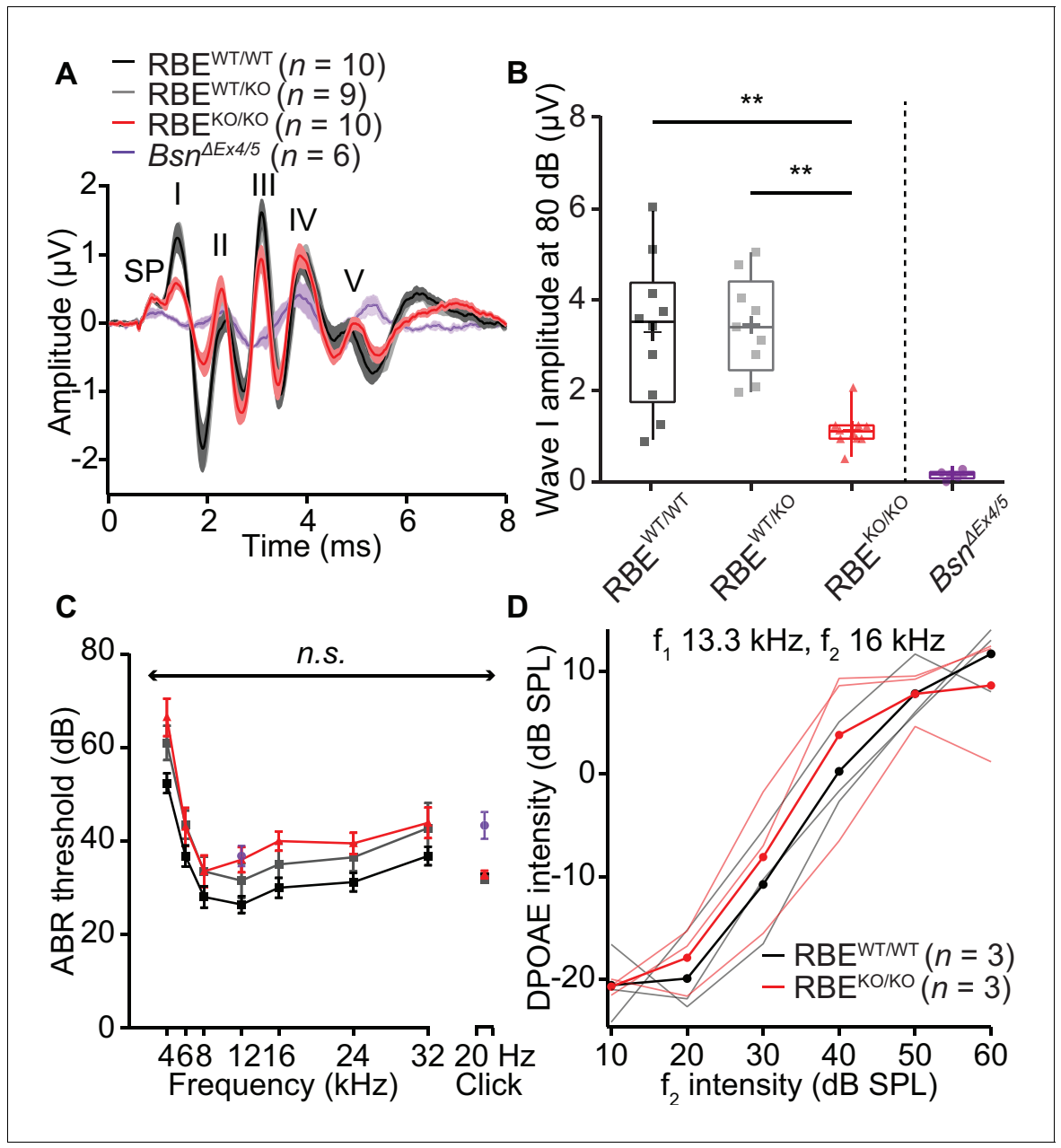

Figure 8. Auditory brainstem responses indicate impaired synchronous $S G N$ activation in $R B E^{K O} / K O$ mice. (A) Average $A B R$ waveforms in response to $80 \mathrm{~dB}$ clicks $\left(n=N=10\right.$ for $R B E^{K O} / K O$ and $R B E^{W T / W T}, N=9$ for RBE ${ }^{W T / K O}$ at 6 weeks of age) showed a reduced amplitude of $A B R$ wave I in $\mathrm{RBE}^{\mathrm{KO} / \mathrm{KO}}$ mice indicating an impairment of SGN activation, which is quantified in (B). This decrease was not as pronounced as in SGNs lacking bassoon (Bsn ${ }^{\Delta E \times 4 / 5}$, data from Jing et al., 2013). The central ABR waves were better preserved, except for wave III. SP: summating potential (hair cell receptor potential), roman numerals (I-V): ABR waves generated along the early auditory pathway. Mean (lines) \pm S.E.M. (shaded areas) are displayed. (B) ABR wave I was significantly reduced in RBE ${ }^{\mathrm{KO} / \mathrm{KO}}$ mice as compared to $\operatorname{RBE}^{\mathrm{WT} / W T}$ and $\operatorname{RBE}^{\mathrm{WT} / K O}$ ( $p=0.0051$ and $p=0.0017$, respectively, NPMC test). No statistical significance was observed between responses recorded in $\mathrm{RBE}^{\mathrm{WT} / \mathrm{WT}}$ and $\mathrm{RBE} \mathrm{WT}^{\mathrm{WTO}}$ mice ( $\mathrm{p}>0.9999, \mathrm{NPMC}$ test). Data from $B s n^{\Delta E \times 4 / 5}$ are shown for comparison. Box plots show 10, 25, 50, 75 and $90^{\text {th }}$ percentiles with the individual data points overlaid, means are shown as crosses. (C) ABR thresholds were comparable in RBE ${ }^{\text {WT/WT }}$, $\mathrm{RBE}^{\mathrm{WT} / \mathrm{KO}}$ and $\mathrm{RBE}{ }^{\mathrm{KO} / \mathrm{KO}}$ for tone burst-driven (n.s., Tukey's test) and click-driven ABRs (n.s., NPMC test). Previously published data for $B s n^{\Delta E \times 4 / 5}$ showed elevated thresholds as a response to short stimuli (click $20 \mathrm{~Hz}$ ) but a similar threshold level at $12 \mathrm{kHz}$. (D) DPOAE amplitude in response to pairs of simultaneous sine waves $\left(f_{1}\right.$ and $f_{2}$, frequencies indicated on panel) at increasing stimulus intensity ( $f_{1}$ intensity $10 \mathrm{~dB}$ above $f_{2}$ in all cases). Mean (thick lines) and data from individual mice ( $n=\mathrm{N}=3$ in $\mathrm{RBE}^{\mathrm{WT} / \mathrm{WT}}$ and $\left.\mathrm{RBE}^{\mathrm{KO} / \mathrm{KO}}\right)$ are displayed.

DOI: https://doi.org/10.7554/eLife.29275.014

The following figure supplement is available for figure 8:

Figure supplement 1. Quantification of $A B R$ waves II- $\mathrm{V}$ amplitude in $\mathrm{RBE}^{\mathrm{KO} / \mathrm{KO}}$ mice. 
Strenzke et al., 2009). We found a non-significant trend of ABR threshold to be increased across all frequencies in $\mathrm{RBE}^{\mathrm{KO} / \mathrm{KO}}$ mice (approximately $10 \mathrm{~dB}$ across all frequencies, Figure $8 \mathrm{C}$; refer to the companion paper Becker et al. showing significantly increased ABR-thresholds based on a larger sample, $\mathrm{N}=28 \mathrm{RBE}^{\mathrm{KO} / \mathrm{KO}}$ mice vs. $22 \mathrm{RBE}^{\mathrm{WT} / \mathrm{WT}}$ mice). Cochlear amplification, probed by recordings of distortion product otoacoustic emissions (DPOAE, Figure $8 D$ ), was intact in $\mathrm{RBE}^{\mathrm{KO} / \mathrm{KO}}$ mice. Additionally, RBE ${ }^{\mathrm{WT} / K O}$ mice showed no significant changes in ABR wave I amplitudes and ABR thresholds (Figure 8), suggesting that the subtle morphological differences observed for afferent synapses of $\mathrm{RBE}^{\mathrm{WT} / \mathrm{KO}}$ IHCs by electron and confocal-immunofluorescence microscopy did not turn into a deficit of sound coding measurable by ABR recordings. The wave I amplitude reduction and ABR threshold elevation were much less pronounced than in bassoon mutant mice (Khimich et al., 2005; Buran et al., 2010; Jing et al., 2013).

We then turned to in vivo extracellular recordings from single auditory neurons by targeting glass microelectrodes to where the auditory nerve enters the anteroventral cochlear nucleus (AVCN) in the brainstem (Taberner and Liberman, 2005; Jing et al., 2013). 'Putative' SGNs (hereafter dubbed SGN for simplicity) were identified based on the depth of electrode position and their firing response to pure-tone stimulation (primary-like peristimulus time histogram and latency, Figure 9) and analyzed in separation from 'putative' cochlear nucleus neurons (Figure 10). Since all firing of the individual SGN is thought to be driven by transmitter release from a single IHC AZ (Heil et al., 2007; Liberman, 1978; Robertson and Paki, 2002), these recordings provide insight into single AZ function. We first assessed the spontaneous firing activity and found an increased abundance of $\mathrm{SGNs}$ with low spontaneous firing rates in $\mathrm{RBE}^{\mathrm{KO} / \mathrm{KO}}$ mice $(72 \%$ with rates $<10 \mathrm{~Hz}, n=43 \mathrm{SGNs}$, $\mathrm{N}=9$ vs. $50 \%$ in RBE ${ }^{\mathrm{WT} / \mathrm{WT}}, n=40 \mathrm{SGNs}, \mathrm{N}=8 ; \mathrm{p}=0.0267$, Kolmogorov-Smirnov test; Figure 9A). Frequency tuning was intact in $\mathrm{RBE}^{\mathrm{KO} / \mathrm{KO}} \mathrm{SGNs}$ (Figure 9B): the sharpness of tuning expressed by the $\mathrm{Q}_{10 \mathrm{~dB}}$ (width of tuning curve $10 \mathrm{~dB}$ above threshold at the characteristic frequency $\left(\mathrm{C}_{\mathrm{f}}\right)$ normalized by $C_{f}$ ) was comparable (mean: $9.28 \pm 1.01$, S.D. $=6.32$ and median: 7.41 for RBE ${ }^{\mathrm{KO} / \mathrm{KO}} \mathrm{SGNs}$, $n=39$ SGNs, $N=9$ vs. mean: $12.50 \pm 1.98$, S.D. $=11.91$ and median: 8.36 for RBE ${ }^{W T / W T}$ SGNs, $n=36$ SGNs, $N=9 ; p=0.28$, Mann-Whitney-Wilcoxon test). However, the sound threshold at $C_{f}$ was significantly elevated by almost $20 \mathrm{~dB}$ in $\mathrm{RBE}{ }^{\mathrm{KO} / \mathrm{KO}}$ mice $(35.60 \pm 3.45 \mathrm{~dB} \mathrm{SPL}, \mathrm{S} . \mathrm{D} .=22.66 \mathrm{~dB} \mathrm{SPL}$ for RBE ${ }^{\mathrm{KO} / \mathrm{KO}} \mathrm{SGNs}, n=43 \mathrm{SGNs}, \mathrm{N}=9$ vs. $16.05 \pm 2.47 \mathrm{~dB}$ SPL, S.D. $=15.42 \mathrm{~dB}$ SPL for RBE ${ }^{\mathrm{WT} / \mathrm{WT}}$ SGNs, $n=39$ SGNs, $N=9, p<0.0001$, Mann-Whitney-Wilcoxon test; Figure 9C). Given the normal frequency tuning and DPOAE, this threshold increase seems unlikely to result from a putative functional cochlear deficit upstream of the IHCs.

Next, we studied the firing response of SGNs to 50 ms tone bursts (at $C_{f}$ and $30 \mathrm{~dB}$ above sound threshold, 200 ms inter-stimulus interval), which is governed by the presynaptic glutamate release and postsynaptic spike generation. The peak firing rate at sound onset is thought to reflect the initial rate of release from the SV-occupied release sites of the RRP ('standing RRP', [Oesch and Diamond, 2011; Pangršič et al., 2012]). Refractoriness and the decline of release rate due to partial depletion of the standing RRP likely dominate the subsequent spike rate adaptation. Finally, the adapted firing rate reports SV replenishment and subsequent fusion (reviewed in Pangršič et al., 2012; Rutherford and Moser, 2016). We observed reduced spike rates of SGNs from RBE ${ }^{\mathrm{KO} / \mathrm{KO}}$ mice (Figure 9D,E) both at sound onset ( $p=0.0001, n=39 \mathrm{SGNs}, \mathrm{N}=8$ in $\mathrm{RBE}^{\mathrm{KO} / \mathrm{KO}}$ and $n=38 \mathrm{SGNs}$, $\mathrm{N}=9$ in RBE ${ }^{W T} / \mathrm{WT}$, t-test) and after short-term adaptation ( $p=0.0023$, Mann-Whitney-Wilcoxon test). Both, peak and adapted rates were similarly affected by the RIBEYE-disruption, indicated by the scatter plot of peak vs. adapted rates (Figure 9E). A significant peak rate reduction was also observed at higher stimulation frequencies $(10 \mathrm{~Hz}$, Figure $9 \mathrm{G}-H)$. The spike rates were better preserved in RBE ${ }^{\mathrm{KO} / \mathrm{KO}} \mathrm{SGNs}$ than in SGNs of bassoon mutant mice (Bsn ${ }^{\Delta e x 4 / 5}$ data of Jing et al. (2013), purple data in Figure 9G-I). We approximated adaptation within the $50 \mathrm{~ms}$ response by singleexponential fitting since double exponential fitting did not regularly report two temporally discernible components in RBE ${ }^{\mathrm{KO} / \mathrm{KO}} \mathrm{SGNs}$. The mean apparent adaptation time constant reported by single-exponential fitting were significantly slowed in $\mathrm{RBE}^{\mathrm{KO} / \mathrm{KO}} \mathrm{SGNs}(9.83 \pm 0.50 \mathrm{~ms}, \mathrm{~S} . \mathrm{D}$. $=2.85 \mathrm{~ms}$, median: $10.46 \mathrm{~ms}, \mathrm{n}=32 \mathrm{SGNs}, \mathrm{N}=8)$ as compared to RBE ${ }^{W T} / \mathrm{WT}$ SGNs $(8.71 \pm 0.50 \mathrm{~ms}, \mathrm{~S}$. D. $=3.05 \mathrm{~ms}$, median: $8.73 \mathrm{~ms}, n=37 \mathrm{SGNs}, \mathrm{N}=9, \mathrm{p}=0.033$, Mann-Whitney-Wilcoxon test). The results of double-exponential fitting of $\mathrm{RBE}^{\mathrm{WT} / \mathrm{WT}}$ and $\mathrm{RBE}^{\mathrm{KO} / \mathrm{KO}} \mathrm{SGNs}$ support the slowed adaptation kinetics and are presented in Table 1. As expected for the reduced peak firing rate, we found prolonged first spike latency which also showed greater temporal jitter (Figure 9F). The reduced peak firing rate together with increased first spike latency jitter likely explain the reduction in $A B R$ 
A

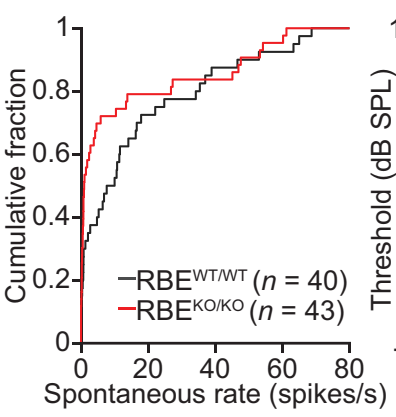

D $50 \mathrm{~ms}$ tone burst $5 \mathrm{~Hz}$

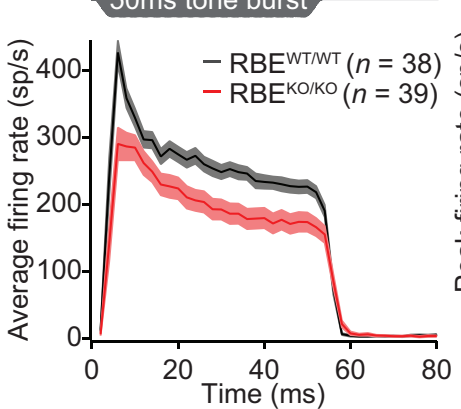

G

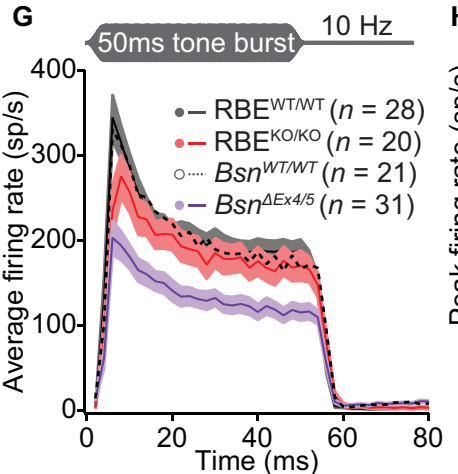

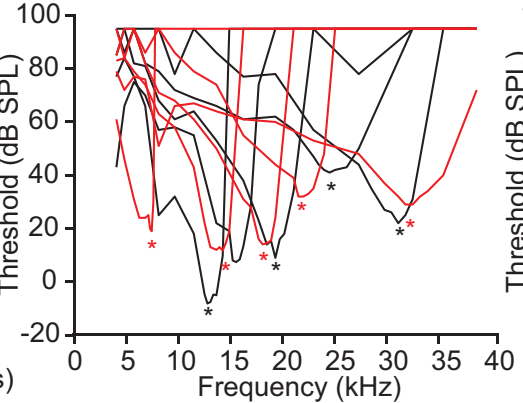

C

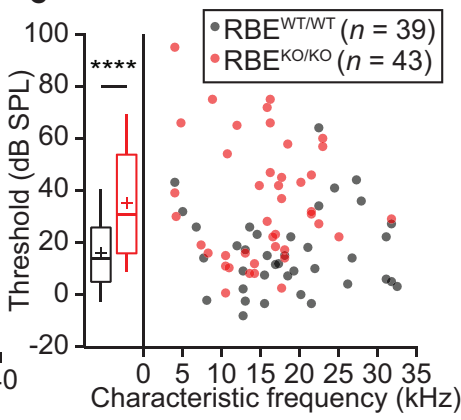

E
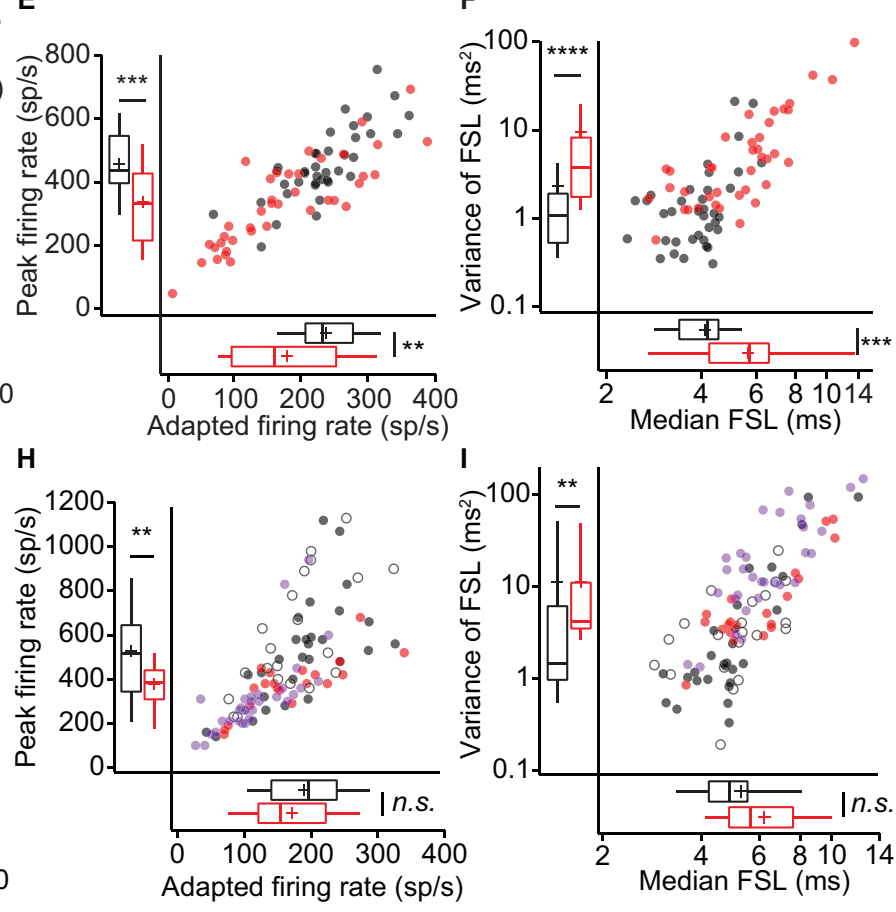

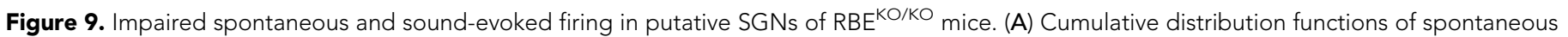

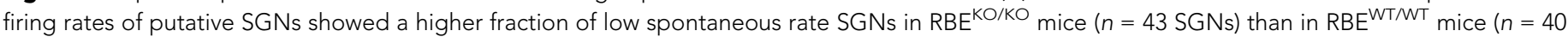

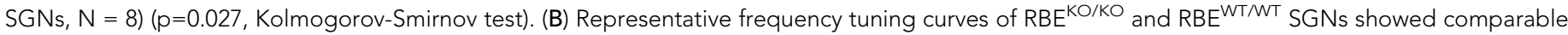

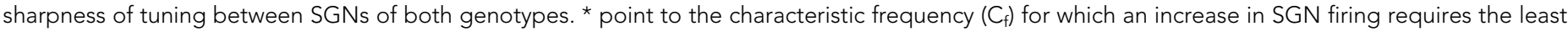
sound pressure level. (C) Thresholds at $\mathrm{C}_{f}$ of RBE ${ }^{\mathrm{KO} / \mathrm{KO}} \mathrm{SGNs}$ ( $n=43 \mathrm{SGNs}, \mathrm{N}=9$ ) were higher than those in RBE ${ }^{W T / W T}$ mice ( $n=39 \mathrm{SGNs}$, $\mathrm{N}=9$ )

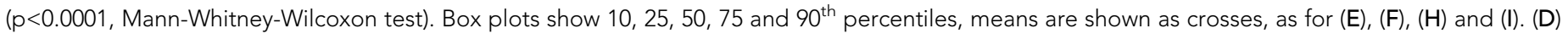

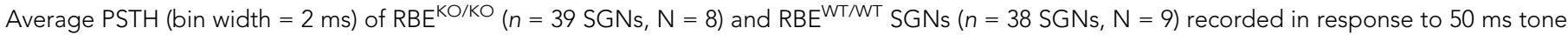
bursts at $\mathrm{C}_{\mathrm{f}} 30 \mathrm{~dB}$ above threshold at a stimulus rate of $5 \mathrm{~Hz}$. The time course of adaptation of RBE ${ }^{\mathrm{KO} / \mathrm{KO}} \mathrm{SGNs}$ (calculated by fitting a single

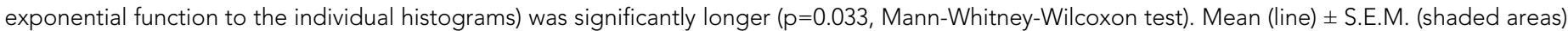
are displayed as for (G). (E) Scatterplot of peak firing rate (bin with highest rate at sound onset) and adapted firing rate (averaged $35-45 \mathrm{~ms}$ from

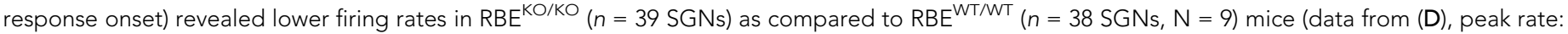
$p=0.0001$, adapted rate: $p=0.0023$, Mann-Whitney-Wilcoxon test). (F) Increased latency (data from (D), $p=0.0002)$ and variance of latency ( $<<0.0001$,

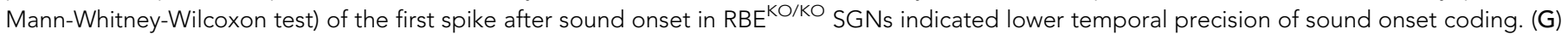

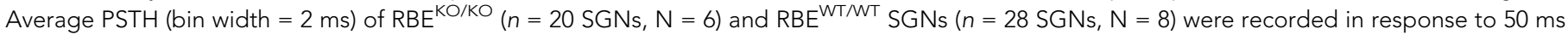
tone bursts at $\mathrm{C}_{\mathrm{f}} 30 \mathrm{~dB}$ above threshold at a stimulus rate of $10 \mathrm{~Hz}$ and showed a similar adapted response in $\mathrm{RBE}$ KO/KO as compared to lower

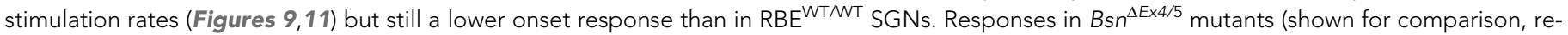

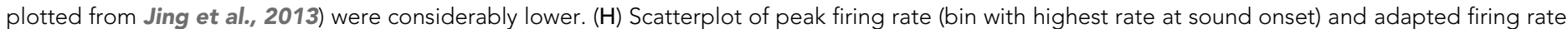
(averaged 35-45 ms from response onset) show decreased onset firing rates in RBE ${ }^{K O} / \mathrm{KO}$ as compared to RBE ${ }^{\mathrm{WT} / \mathrm{WT}}$ mice (data from (G), $\mathrm{p}=0.0093$,

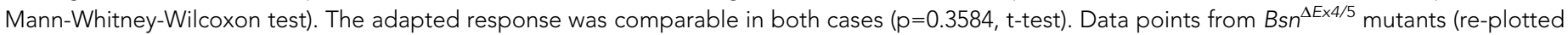

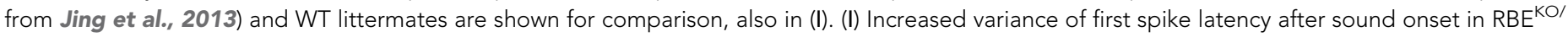
Figure 9 continued on next page 
Figure 9 continued

KO SGNs ( $p=0.0089$, Mann-Whitney-Wilcoxon test) and comparable latencies were observed at this stimulation frequency (data from (G), $p=0.0761$, Mann-Whitney-Wilcoxon test).

wave I amplitude. The firing of putative AVCN neurons was better preserved: putative bushy cells showed normal sound driven rates and chopper cells only a mild reduction in peak rate (Figure 10).

Next, we explored the encoding of sound intensity by estimating the mean firing rate during 50 $\mathrm{ms}$ tone bursts at different sound pressure levels. These 'rate-level functions' (Figure 11A) indicated that the spike rate increase with the sound pressure level ( $p=0.068, n=24 \mathrm{SGNs}, \mathrm{N}=8$ in RBE $\mathrm{KO}^{\mathrm{KOO}}$ and $n=19$ SGNs, $N=7$ in RBE ${ }^{W T / W T}$, Mann-Whitney-Wilcoxon test, Figure 11-figure supplement $1 \mathrm{~A}$ ) and the dynamic range of sound coding (sound pressure level for which the spike rate changes from 10-90\%, Figure 11-figure supplement 1B, $p=0.3044$, t-test) were not significantly altered. We then used transposed tones $\left(C_{f}\right.$ at $500 \mathrm{~Hz}$ modulation frequency) in order to probe for the temporal fidelity and reliability of firing in $\mathrm{RBE}^{\mathrm{KO} / \mathrm{KO}} \mathrm{SGNs}$ in the steady state (Figure 11D). These experiments corroborated the reduced maximal firing rate of $\mathrm{RBE}^{\mathrm{KO} / \mathrm{KO}} \mathrm{SGNs}(n=22 \mathrm{SGNs}, \mathrm{N}=7$ in $\mathrm{RBE}^{\mathrm{KO} / \mathrm{KO}}$ and $n=15 \mathrm{SGNs}, \mathrm{N}=6$ in $\mathrm{RBE}^{\mathrm{WT} / \mathrm{WT}}, \mathrm{p}<0.0001$, t-test) and indicated that the temporal precision of sound coding is impaired also in the steady state (reduced Synchronization Index: $p=0.0043$, t-test).

In order to further scrutinize the potential role of the synaptic ribbon in vesicle replenishment, we studied the response to prolonged tone-stimulation (Figure $11 \mathrm{~B}, 500 \mathrm{~ms}$ at $\mathrm{C}_{\mathrm{f}}$ and $30 \mathrm{~dB}$ above threshold, 2 s inter-stimulus interval). The peak rate was better preserved in RBE ${ }^{K O} / K O S G N$ s than

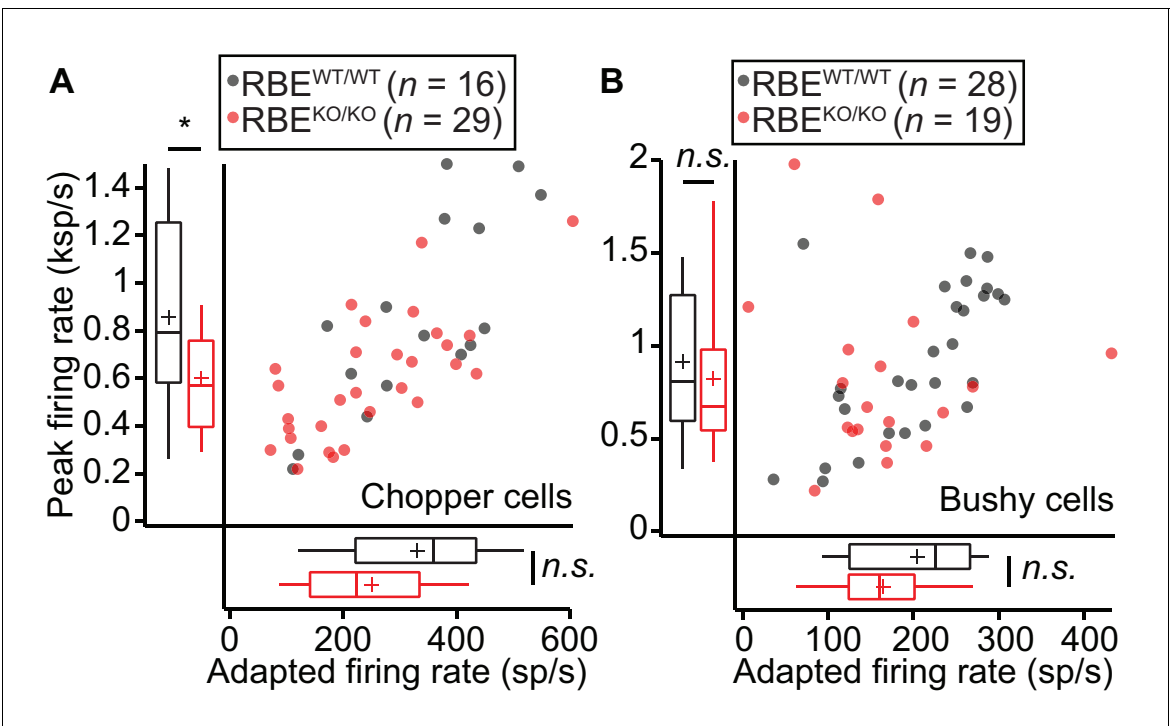

Figure 10. Responses to pure tones in cochlear nucleus cells showed that the mutant phenotype is partially compensated in higher stations of the auditory pathway. (A) Quantification of peak and adapted responses to 50 ms tone burst stimulation, $30 \mathrm{~dB}$ above threshold at $\mathrm{C}_{\mathrm{f}}$ in $\mathrm{SGNs}$ displaying a chopper discharge pattern (periodically alternating phases of high firing rates with low firing rates, typical of multipolar cells in the posterior ventral cochlear nucleus), showed that the differences in rate faded away opposite to those shown at the level of the auditory nerve. Peak rates were still significantly lower in $\mathrm{RBE}^{\mathrm{KO} / \mathrm{KO}}(n=29 \mathrm{SGNs}, \mathrm{N}=9)$ as compared to $\operatorname{RBE}^{W T / W T}(n=16 \mathrm{SGNs}, \mathrm{N}=9$ ) mice ( $p=0.0303$, Mann-Whitney-Wilcoxon test), while the adapted rate showed a non-significant trend towards reduction in $\operatorname{RBE}^{\mathrm{KO} / \mathrm{KO}}\left(\mathrm{p}=0.0538\right.$, t-test). Box plots show $10,25,50,75$ and $90^{\text {th }}$ percentiles and means are shown as crosses, as for (B). (B) Same recordings paradigm as (A) performed in a fiber with bushy cell discharge pattern (similar to the one found in SGNs, typical also in this type of cochlear nucleus neurons) showed comparable responses in both peak ( $p=0.2601$, Mann-Whitney-Wilcoxon test) and adapted rate ( $p=0.0510$, Mann-Whitney-Wilcoxon test) in $\operatorname{RBE}^{\mathrm{KO} / \mathrm{KO}}(n=19 \mathrm{SGNs}, \mathrm{N}=7)$ and RBE ${ }^{\mathrm{WT} / \mathrm{WT}}(n=28 \mathrm{SGNs}, \mathrm{N}=10)$. DOI: https://doi.org/10.7554/eLife.29275.017 


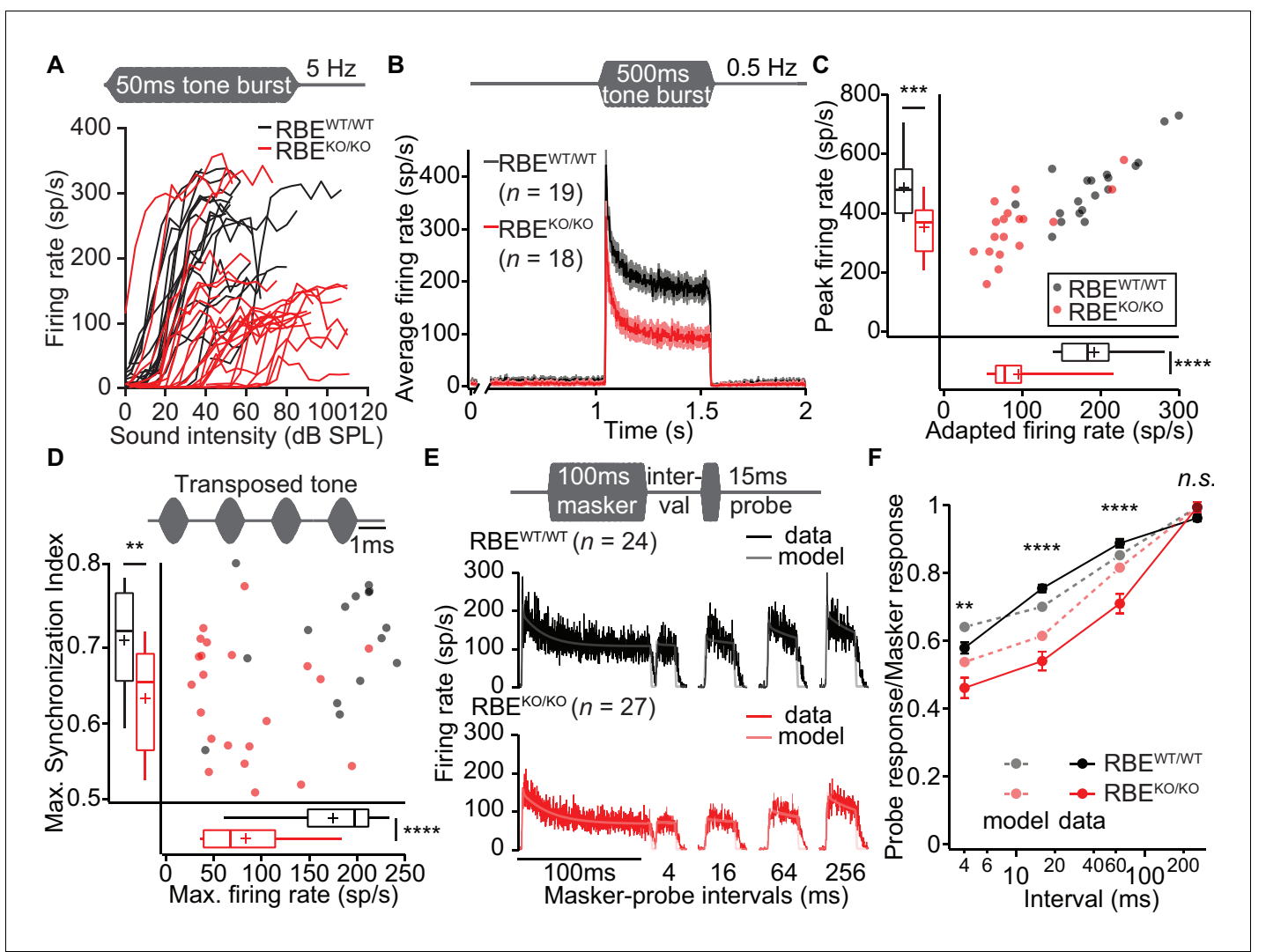

Figure 11. Lack of ribbons impairs vesicle replenishment in $\mathrm{RBE}^{\mathrm{KO} / \mathrm{KO}}$ mice. (A) Intensity coding was largely preserved for suprathreshold sound stimulation: rate-level functions (average increase in spike rate with stimulus intensity) of SGNs in response to 50 ms tone bursts at $\mathrm{C}_{\mathrm{f}}$ at $5 \mathrm{~Hz}$ corroborated the notion of increased sound threshold but were otherwise comparable in $\operatorname{RBE}^{\mathrm{KO} / \mathrm{KO}}(n=24 \mathrm{SGNs}, \mathrm{N}=8)$ and RBE ${ }^{\mathrm{WT} / \mathrm{WT}}$ ( $n=19 \mathrm{SGNs}$, $\mathrm{N}=7$ ) mice, for quantification see Figure 11-figure supplement $1 \mathrm{C}$. (B) Average PSTH recorded in response to $50 \times 500 \mathrm{~ms}$ tone bursts at $\mathrm{C}_{f}$ and 30 $\mathrm{dB}$ above threshold at a stimulus rate of $0.5 \mathrm{~Hz}$ (bin width $=2 \mathrm{~ms}$ ): the onset response to this stimulus was preserved better in $\mathrm{RBE}{ }^{\mathrm{KO} / \mathrm{KO}} \mathrm{SGNs}(n=18$ $\mathrm{SGNs}, \mathrm{N}=7$ ) as compared to higher stimulation rates (Figure 9), but it was still lower than in RBEWT/WT SGNs $(n=19 \mathrm{SGNs}$, $\mathrm{N}=7$ ), as well as the adapted firing rate. Mean (line) \pm S.E.M. (shaded areas) are displayed. (C) Scatterplot of peak (highest 2 ms bin) and adapted (averaged $405-415$ ms from response onset) firing rates: significantly reduced peak ( $p=0.0005$, t-test) and adapted ( $<<0.0001$, Mann-Whitney-Wilcoxon test) rates in RBE ${ }^{K O} / K O$ SGNs. Box plots show 10, 25, 50, 75 and $90^{\text {th }}$ percentiles and means are shown as crosses, as for (D). (D) Temporal precision and reliability of sound coding is impaired in RBE ${ }^{\mathrm{KO} / \mathrm{KO}} \mathrm{SGN}$ s. Synchronization of firing to stimulus and firing rates (reflecting spike probability) were reduced when probed with amplitude-modulated (transposed) tones (continuous stimulation with a carrier frequency at $\mathrm{C}_{\mathrm{f}}$ and at a modulation frequency of $500 \mathrm{~Hz}$ ) ( $p=0.0043$, t-test, for synchronization index, and $p<0.0001$, t-test, for firing rate, when comparing RBE ${ }^{\mathrm{KO} / \mathrm{KO}}(n=22 \mathrm{SGNs}, \mathrm{N}=7)$ and RBE ${ }^{\mathrm{WT} / \mathrm{WT}}$ ( $n=15$ $\mathrm{SGNs}, \mathrm{N}=6$ ) SGNs). (E) Forward masking experiments were used to study presynaptic SV dynamics: a stimulus complex consisting of a 100 ms masker stimulus, a silent interval of 4/16/64/256 ms and a $15 \mathrm{~ms}$ probe (both at the characteristic frequency, $30 \mathrm{~dB}$ above threshold) was presented at $2 \mathrm{~Hz}$. The averaged data after alignment of $\mathrm{RBE}^{\mathrm{WT} / \mathrm{NT}}\left(n=24 \mathrm{SGNs}, \mathrm{N}=7\right.$, black) and RBE ${ }^{\mathrm{KO} / \mathrm{KO}}(n=27 \mathrm{SGNs}, \mathrm{N}=8$, red) SGNs responses are displayed as described in Materials and methods. On top of the data we present a fit of a biophysical model (light gray and pink lines) to the data used to study the SV dynamics at the AZ. The refilling and fusion rate constant during spontaneous and stimulated conditions as well as the number of occupied release sites are provided in Table 2. (F) Recovery of onset response showed as ratio of probe and masker response (number of spikes during first 10 ms, mean \pm S.E.M), and prediction (dashed lines) derived from the model fit shown in (E). Recovery was slower in RBE ${ }^{\mathrm{KO} / \mathrm{KO}} \mathrm{SGNs}(n=27 \mathrm{SGNs}, \mathrm{N}=8)$ as compared to RBE ${ }^{W T} / W T$ ( $n=24$ SGNs, $N=7$ ) with significant differences in the ratio after $4 \mathrm{~ms}(p=0.0019, t$-test), 16 ms ( $p<0.0001$, Mann-WhitneyWilcoxon test), and $64 \mathrm{~ms}$ masker-probe intervals ( $p<0.0001$, Mann-Whitney-Wilcoxon test), but not after $256 \mathrm{~ms}$ ( $p=0.0835, t$-test).

DOI: https://doi.org/10.7554/eLife.29275.019

The following figure supplement is available for figure 11:

Figure supplement 1. Rate-level functions and dynamic range remained unchanged in $\mathrm{RBE}^{\mathrm{KO} / \mathrm{KO}}$

DOI: https://doi.org/10.7554/eLife.29275.020

seen with shorter inter-stimulus interval (e.g. 200 ms, Figure 9C), likely reflecting more complete SVreplenishment (i.e. larger standing RRP) owing to the longer recovery interval ( $2 \mathrm{~s}$ vs. $200 \mathrm{~ms}$ ). However, the adapted spike rate of $\mathrm{RBE}^{\mathrm{KO} / \mathrm{KO}} \mathrm{SGNs}$ was even more reduced than found with $50 \mathrm{~ms}$ tone bursts (to about half of that for RBEWT/WT SGNs, Figure 11B,C) highlighting the impaired SV 
Table 1. Average double-exponential fitting results to peristimulus time histograms obtained by 50 ms tone bursts $30 \mathrm{~dB}$ above threshold at $\mathrm{C}_{\mathrm{f}}(200 \mathrm{~ms}$ inter-stimulus interval).

\begin{tabular}{llllll} 
& Fast time constant & Slow time constant & Amplitude fast component & Amplitude slow component & R \\
\hline RBE $^{\text {WT/WT }}$ & $6.31 \pm 0.77$ & $95.63 \pm 24.21$ & $484.61 \pm 104.76$ & $58.42 \pm 12.90$ & $0.93 \pm 0.00$ \\
\hline RBE $^{\text {KO/KO }}$ & $18.79 \pm 6.91$ & $101.66 \pm 27.77$ & $310.56 \pm 73.60$ & $64.92 \pm 107.49$ \\
\hline p-value & 0.0045 & 0.3580 & 0.0519 & 0.5475 & $0.91 \pm 0.01$ \\
\hline
\end{tabular}

replenishment during prolonged stimulation. Finally, we evaluated presynaptic vesicle pool dynamics by recording and modeling responses to forward masking protocols (Harris and Dallos, 1979), that are thought to reflect depletion and recovery of the RRP (Figure 11E,F; Figure 11-figure supplement $1 \mathrm{C}$ ). We approximated the recovery from forward masking by single exponential fitting (Figure 11-figure supplement $1 \mathrm{C})$ to provide an estimate of the kinetics of vesicle pool replenishment. The time constant of recovery was prolonged in RBE ${ }^{K O} / K O S G N s(90.80 \pm 8.66 \mathrm{~ms}, \mathrm{~S}$. D. $=45.00 \mathrm{~ms}, n=27 \mathrm{SGNs}, \mathrm{N}=8$ vs. $33.53 \pm 5.74 \mathrm{~ms}, \mathrm{~S} . \mathrm{D} .=28.11 \mathrm{~ms}$, in RBE ${ }^{\mathrm{WT}} / \mathrm{WT} \mathrm{SGNs}, n=24$ SGNs, $N=7, p<0.0001$, Mann-Whitney-Wilcoxon test) indicating slowed RRP replenishment in the absence of the ribbon. We noted that SGNs showed considerably lower spontaneous and evoked rates during the forward masking paradigm compared to other stimulus protocols (compare Figure 11B E), likely due to enhanced RRP depletion with the more extended stimulation in this protocol.

Amplitude and waveform of the forward masking responses were consistent with a two-fold reduction of the number of contributing vesicular release sites of the RRP ( $\mathrm{N}_{\text {slot }}$ : contributing release sites during forward masking, $\mathrm{N}_{\text {slot }}$ : the contributing release sites for the same $A Z$ s during tone bursts at $5 \mathrm{~Hz}$ stimulation). The ratio $\mathrm{N}_{\text {slots }} / \mathrm{N}^{\prime}$ slots was estimated to be 2.2 for $\mathrm{RBE}{ }^{\mathrm{KO} / \mathrm{KO}}$ and 2.3 for $\mathrm{RBE}^{\mathrm{WT} / W T}$ from the drop in spontaneous and evoked SGN spiking rates. We used a previously developed biophysical model of RRP dynamics and spike generation (Frank et al., 2010; Jung et al., $2015 b)$ to extract information on fusion and replenishment rate constants as well as the $\mathrm{N}_{\text {slot }}$ by fitting the responses to tone bursts, that is PSTHs with 100, 200 and 2000 ms inter-stimulus interval, and also the forward masking spiking data across all recovery intervals (Figure 11E). Importantly, only the first $50 \mathrm{~ms}$ of the 500 ms stimulus response during the PSTH 2000 were included in the fit; any later adaptation processes were disregarded, as they were not accounted by the model's equations.

The results of model fitting suggested that during the forward masking only about half of all release sites $\left(\mathrm{N}_{\text {slots }}\right)$ were engaged in the response $\left(\mathrm{N}^{\prime}{ }_{\text {slots }}\right)$. Throughout, $\mathrm{RBE}^{\mathrm{KO} / \mathrm{KO}} \mathrm{SGNs}$ showed a lower fusion rate than the RBE ${ }^{\mathrm{WT} / \mathrm{WT}} \mathrm{SGNs}$, reflecting the reduced onset response in RBE $\mathrm{KO}^{\mathrm{K} O}$ SGNs. When more recovery time was allowed, that is in the $0.5 \mathrm{~Hz}$ tone burst and the forward masking, where recovery times from around 250 to $500 \mathrm{~ms}$ occured between the probe and subsequent masker, the onset response improved in $\mathrm{RBE}^{\mathrm{KO} / \mathrm{KO}}$. Consequently, the estimated fusion rate almost reached the level of RBE ${ }^{W T} / W T$ in the forward masking fits. With the scaling factors of approximately 2 , the estimates for the number of release sites were consistent between tone bursts and forward

Table 2. Parameters for the biophysical model capturing the release dynamics during forwardmasking and repetitive tone burst experiments

\begin{tabular}{|c|c|c|c|c|}
\hline & \multicolumn{2}{|c|}{ Forward masking } & \multicolumn{2}{|c|}{ All tone bursts - global fit } \\
\hline & $\mathrm{RBE}^{\mathrm{WT}} / \mathrm{WT}$ & $\mathrm{RBE}^{\mathrm{KO} / \mathrm{KO}}$ & $\mathrm{RBE}^{\mathrm{WT} / \mathrm{WT}}$ & $\mathrm{RBE}^{\mathrm{KO} / \mathrm{KO}}$ \\
\hline $\mathrm{N}_{\text {slots }}$ & 8 & 6.5 & 15.6 & 13.7 \\
\hline$k_{\text {refill, stim }}$ & 29.5 & 18.2 & 18.4 & 19.9 \\
\hline$k_{\text {refill, spont }}$ & 13.3 & 7.1 & 5.59 & 2.71 \\
\hline $\mathrm{k}_{\text {fusion, stim }}$ & 32.2 & 31 & 82.5 & 45.1 \\
\hline $\mathrm{k}_{\text {fusion, spont }}$ & 0.6 & 0.9 & 0.63 & 0.54 \\
\hline
\end{tabular}

DOI: https://doi.org/10.7554/eLife.29275.021 
masking data, and in both cases only slightly smaller for the RBE ${ }^{\mathrm{KO} / \mathrm{KO}}$ (Table 2). Taken together the fits from forward masking and tone bursts suggest that the total number of release sites (RRP) was only slightly reduced at ribbonless synapses of $\mathrm{RBE} \mathrm{KO}^{\mathrm{KOO}} \mathrm{IHCs}$. However, a strong firing response at sound onset, that is release of a large standing RRP, required longer recovery indicating more efficient SV replenishment in the presence of ribbons, which is reflected in the larger refilling rate constants estimated by the model of RBE ${ }^{\mathrm{WT} / \mathrm{WT}}$ synapses.

\section{Discussion}

The role of the synaptic ribbon has remained a topic of intense research. Here, we studied the structure and function of IHC afferent synapses with SGNs in mice lacking RIBEYE, the core component of the synaptic ribbon. Morphologically, synapses of $\mathrm{RBE}^{\mathrm{KO} / \mathrm{KO}} \mathrm{IHCs}$ did not simply lack ribbons but instead appeared transformed to contacts where release from multiple AZ feeds into one postsynaptic bouton. Synaptic transmission was impaired at the ribbonless IHC synapses of RBE ${ }^{\mathrm{KO} / K O}$ mice. Spontaneous SGN firing was reduced, sound-evoked firing had higher sound thresholds, lower peak and adapted rates, recovered more slowly from adaptation and had a greater temporal jitter. Modelling of synaptic sound encoding corroborated the notion of reduced rates of SV fusion and replenishment at the ribbonless synapses of $\mathrm{RBE}^{\mathrm{KO} / \mathrm{KO}}$ mice. Analysis of IHC function revealed a small depolarized shift in the operating range of the synaptic Cav1.3 $\mathrm{Ca}^{2+}$-channels, which likely contributes to the reduced spontaneous and evoked firing rates and to the elevated sound thresholds of $\mathrm{RBE}^{\mathrm{KO} / \mathrm{KO}} \mathrm{SGNs} . \Delta \mathrm{C}_{\mathrm{m}}$ recordings revealed a mild reduction of exocytosis but only for weaker depolarizations, which we primarily attribute to the depolarized shift of $\mathrm{Ca}^{2+}$-channel activation. In summary, our data support a role of the ribbon in vesicle replenishment and $\mathrm{Ca}^{2+}$-channel regulation at the AZs as required for synchronous activation of SGNs in normal hearing. However, our analysis of $\mathrm{RBE}^{\mathrm{KO} / \mathrm{KO}}$ mice likely underestimated the role of the ribbon due to substantial compensation that is best illustrated by the striking transformation of $A Z$ morphology and the mild ex vivo phenotype of $\mathrm{IHC} \mathrm{Ca}{ }^{2+}$-influx and exocytosis.

\section{RIBEYE deletion transforms IHC synapses to 'conventional-like' presynaptic ultrastructure, where multiple ribbonless AZs collectively maintain large complements of $\mathrm{Ca}^{2+}$-channels and SVs}

Our work confirms the central role of RIBEYE for forming synaptic ribbons (Schmitz et al., 2000; Magupalli et al., 2008; Maxeiner et al., 2016). We did not observe structures reminiscent of 'ghost ribbons' reported for ribeye mutants in zebrafish neuromast hair cells (Lv et al., 2016) in IHCs of $\mathrm{RBE}^{\mathrm{KO} / \mathrm{KO}}$ mice. These ghost ribbons were characterized as a halo of synaptic vesicles around a nonelectron-dense area that resembled in size, though smaller, and shape to a synaptic ribbon. In zebrafish, two gene copies of ribeye (ribeye $a$ and b) exist, making it harder to achieve a complete knockout (Lv et al., 2016; Van Epps et al., 2004). In keeping with this notion, Lv et al. found residual immunofluorescence of ribeye $a$ in the double mutants. Hence, we speculate that residual RIBEYE, possibly together with other scaffold proteins such as piccolo, might have formed the observed electron-translucent SV-framed structures (Lv et al., 2016). In contrast, immunofluorescence, as well as electron microscopy, revealed the complete absence of RIBEYE and ribbons in IHCs of RBE ${ }^{\mathrm{KO} / K O}$ mice in our work and the companion study (Becker et al.), which is in agreement with findings in the $\mathrm{RBE}^{\mathrm{KO} / \mathrm{KO}}$ mouse retina (Maxeiner et al., 2016).

IHC synapses normally employ a single ribbon-type AZ. But in the absence of RIBEYE, there were typically two or more ribbonless AZs, akin to multiple conventional AZs (Figure 2). These ribbonless 'conventional' $A Z s$ at RBE ${ }^{K O / K O} I H C$ synapses consist mostly of roundish PDs, each with a cluster of SVs, of which approximately one third were directly adjacent to the plasma membrane (membraneproximal: MP-SVs). Using electron tomography we found that about two-thirds of the MP-SVs were tethered to the AZ membrane, which was comparable to RBE ${ }^{\mathrm{WT}} / \mathrm{WT}$ AZs (Figure 3). We speculate that SVs associated with the PD, but not facing the membrane (PDA-SVs), serve to replenish the release sites once tethered MP-SVs fused, and that the ribbonless PD more likely acts in long-range SV tethering to the AZ in analogy to what is considered for conventional AZs (Cole et al., 2016; Fernández-Busnadiego et al., 2013; Siksou et al., 2007). We assume that absence of RIBEYE does not alter SV size since electron tomography, which provides the most reliable estimation of SV size, did not reveal differences in SV diameter between $\mathrm{RBE}^{\mathrm{KO} / K O}$ and $\mathrm{RBE}^{\mathrm{WT} / \mathrm{WT}} \mathrm{AZs}$, at least when 
considering all SVs. The RBE ${ }^{\mathrm{KO} / K O} \mathrm{PDs}$, like in RBE ${ }^{\mathrm{WT} / \mathrm{WT}}$, contained bassoon, Cav1.3, and RIM2, but lacked piccolino which is likely part of the ribbon in RBEWT/WT (Figure 1) (Dick et al., 2001; Khimich et al., 2005; Limbach et al., 2011; Regus-Leidig et al., 2013).

To some extent, the multi-AZ morphology is reminiscent of IHC synapses prior to synaptic maturation (Huang et al., 2012; Sendin et al., 2007; Sobkowicz et al., 1982; Wong et al., 2014). While we cannot rule out some sort of developmental delay of RIBEYE-deficient IHCs, we suspect that the morphological transformation into a multi-AZ morphology reflects a compensatory effort. Reasons for our interpretation include (i) the same morphological phenotype of RBE ${ }^{\mathrm{KO} / \mathrm{KO}} \mathrm{IHCs}$ at 8 months of age (Figure 2), (ii) the finding of highly regular PDs at RBE ${ }^{\mathrm{KO} / K O} \mathrm{IHC}$ synapses, which differs from less well-defined PDs at immature AZs (Wong et al., 2014), (iii) the typical continuous and large PSD of RBE ${ }^{\mathrm{KO} / \mathrm{KO}} \mathrm{IHCs}$ synapses (Figure 2, see also the accompanying paper by Becker et al.) as a characteristic of a mature synapse, rather than the several smaller PSD patches at developing IHC synapses (Wong et alo, 2014), and (iv) the synaptically confined Cav1.3 $\mathrm{Ca}^{2+}$-channel clusters, normal amplitude of $\mathrm{IHC} \mathrm{I}_{\mathrm{Ca}}$ and mature amplitude of synaptic $\mathrm{Ca}^{2+}$-signals, rather than massive extrasynaptic Cav1.3 abundance and larger whole-cell $\mathrm{I}_{\mathrm{Ca}}$ but smaller synaptic $\mathrm{Ca}^{2+}$-signals in immature $\mathrm{IHCs}$ (Wong et al., 2014; Zampini et al., 2010). The multi-AZ morphology of the RBE ${ }^{\mathrm{KO} / K O}$ IHC synapses was also corroborated by high- and super-resolution microscopy of bassoon and Cav1.3 immunofluorescence (Figure 4). The organization in several smaller $\mathrm{Ca}^{2+}$-channel clusters likely explains the broader spread of the presynaptic $\mathrm{Ca}^{2+}$-signal at $\mathrm{RBE}^{\mathrm{KO} / \mathrm{KO}}$ synapses (Figure 6). In contrast to bassoon mutant mice (Frank et al., 2010; Jing et al., 2013), the number of synaptic $\mathrm{Ca}^{2+}$-channels was not reduced in $\mathrm{RBE}^{\mathrm{KO} / \mathrm{KO}}$ mice as shown by normal amplitudes of whole-cell $\mathrm{I}_{\mathrm{Ca}}$ and synaptic $\mathrm{Ca}^{2+}$ signals. Therefore, the loss of synaptic $\mathrm{Ca}^{2+}$-channels from the bassoon-deficient ribbonless IHC synapses, indicates a role of bassoon in promoting $\mathrm{Ca}^{2+}$-channel tethering at the $\mathrm{AZ}$ likely via interaction with RIM-binding protein (Davydova et al., 2014), which was previously shown to interact with Cav1.3 $\mathrm{Ca}^{2+}$-channels (Hibino et al., 2002) and is required for establishing a normal $\mathrm{Ca}^{2+}$-channel complement of the IHC AZ (Krinner et al., 2017).

Interestingly, we observed changes in $\mathrm{Ca}^{2+}$-channel function in $\mathrm{RBE}^{\mathrm{KO} / \mathrm{KO}} \mathrm{IHCs}$ : the voltagedependence of $\mathrm{Ca}^{2+}$-channel activation was slightly, but significantly, shifted to more depolarized potentials both at the levels of whole-cell $\mathrm{Ca}^{2+}$-current $\left(\mathrm{V}_{\mathrm{h}}+2 \mathrm{mV}\right)$ and synaptic $\mathrm{Ca}^{2+}$-influx at individual synapses $\left(\mathrm{V}_{\mathrm{h}}+5 \mathrm{mV}\right.$ ) (at $5 \mathrm{mM}\left[\mathrm{Ca}^{2+}\right]_{\mathrm{e}}$, Figure 5 and 6). Similar as in this study, an enhanced inactivation (Figure 5) of $\mathrm{I}_{\mathrm{Ca}}$ was also found in bassoon-deficient $\mathrm{IHCs}$, while their $\mathrm{V}_{\mathrm{h}}$ was actually mildly shifted in the opposite direction $\left(-3 \mathrm{mV}\right.$ for imaging of synaptic $\left.\mathrm{Ca}^{2+}\right)$ and unaltered at the level of the whole-cell $\mathrm{I}_{\mathrm{Ca}}$ (Frank et al., 2010). One potential reason for why the depolarized $\mathrm{V}_{\mathrm{h}}$-shift of the synaptic $\mathrm{Ca}^{2+}$-influx was greater than that of the whole-cell $\mathrm{Ca}^{2+}$-influx is the contribution of extrasynaptic $\mathrm{Ca}^{2+}$-channels to the whole-cell $\mathrm{Ca}^{2+}$-influx. They are thought to contribute approximately $30 \%$ of the $\mathrm{Ca}^{2+}$-influx (Brandt et alo, 2005) and are not regulated by RIBEYE/ribbon. In order to test whether the depolarized $\mathrm{V}_{\mathrm{h}}$-shift of synaptic $\mathrm{Ca}^{2+}$ translates into changes in transmitter release, we recorded exocytic $\Delta \mathrm{C}_{\mathrm{m}}$ for different depolarization potentials. A small, but significant reduction of exocytosis for weak depolarizations in $\mathrm{RBE}^{\mathrm{KO} / \mathrm{KO}} \mathrm{IHCs}$ (Figure 7, seen also in the accompanying paper by Becker et al.) suggests that the $V_{h}$ shift is relevant for hair cell transmission (see also below). How enhanced $\mathrm{I}_{\mathrm{Ca}}$ inactivation might affect sound encoding is addressed by work on $\mathrm{Ca}^{2+}$-binding proteins (CaBPs) that are thought to antagonize calmodulin's role in mediating $\mathrm{I}_{\mathrm{Ca}}$ inactivation (Lee et al., 1999; Peterson et al., 1999). Among the several CaBPs expressed in IHCs (Cui et al., 2007; Picher et al., 2017; Schrauwen et al., 2012; Yang et al., 2006), CaBP2 is defective in human genetic hearing loss DFNB93 (Picher et al., 2017; Schrauwen et al., 2012) and required for hearing likely via inhibition of $\mathrm{IHC} \mathrm{I}_{\mathrm{Ca}}$ inactivation (Picher et alı, 2017). However, deletion of CaBP4 in mice caused only a very mild increase of $\mathrm{I}_{\mathrm{Ca}}$ inactivation similar to the one found here and did not alter auditory brainstem responses (Cui et al., 2007). Future studies need to address how RIBEYE/ribbons mechanistically regulate the function and spatial organization of $\mathrm{Ca}^{2+}$ channels.

\section{What can the RIBEYE knock-out tell us about the function of the ribbon in sensory coding?}

Over some decades, research on retinal photoreceptors and bipolar cells, on hair cells of the inner ear and the lateral lines, on electroreceptors as well as pineal cells, has aimed to elucidate the function(s) of the synaptic ribbon. Current hypotheses state that the ribbon functions in (i) replenishing 
release sites ([Bunt, 1971; Frank et alı, 2010; von Gersdorff et alı, 1996; Lenzi et al., 2002; Maxeiner et al., 2016; Snellman et al., 2011; Vaithianathan and Matthews, 2014] for a deviating view see \{Jackman et al., 2009]), potentially by facilitated diffusion of SVs on the ribbon surface towards the site of consumption (Graydon et al., 2014) and SV priming (Grabner and Zenisek, 2013; Snellman et al., 2011), (ii) establishing a large complement of vesicular release sites and $\mathrm{Ca}^{2+}$-channels at the active zone (Frank et al., 2010; Khimich et al., 2005), which remained hard to disentangle from potential function of bassoon (Frank et al., 2010; Jing et al., 2013), (iii) ensuring close spatial coupling of $\mathrm{Ca}^{2+}$-channels and vesicular release sites (Maxeiner et al., 2016) or enhancing presynaptic $\mathrm{Ca}^{2+}$-signals by limiting diffusional $\mathrm{Ca}^{2+}$-spread (Graydon et al., 2011), (iv) contributing to multivesicular release (Graydon et al., 2011; Jing et al., 2013; Mehta et al., 2013), and (v) contributing to SV reformation from endocytosed membranes (Jung et alı, 2015b; Khimich et al., 2005; Schwarz et al., 2011). Clearly, SV-replenishment was impaired at the ribbon-less IHC synapses of RBE ${ }^{\mathrm{KO} / \mathrm{KO}}$ mice. This is shown by slowed recovery from forward-masking and the use-dependent reduction of peak and adapted firing rates, which we further scrutinized by modeling. Therefore, our study supports a role of the ribbon in vesicle replenishment, which is also found in the accompanying paper by Becker et al.. Why RRP-recovery was not significantly altered when probed with pairs 20 ms long maximal $\mathrm{Ca}^{2+}$-influx by membrane capacitance measurements in IHCs (Figure 7) will need to be addressed in future studies, ideally using paired pre-and postsynaptic recordings of synaptic transmission with depolarizations of varying strength.

Each of the ribbon-manipulations employed to analyze its role has strengths, but also weaknesses, such as changes in other AZ proteins and long-term compensatory processes (e.g. bassoon deletion), complex manipulations (e.g. diurnal changes or hibernation: [Hull et al., 2006; Mehta et alı, 2013; Spiwoks-Becker et al., 2004]) and photoablation (Mehta et al., 2013; Snellman et al., 2011). Genetic RIBEYE manipulations (Lv et al., 2016; Maxeiner et al., 2016; Sheets et al., 2011; Van Epps et al., 2004) have the greatest molecular specificity, but in some cases, were incomplete, and to some extent masked by compensation. In fact, our study of IHCs, unlike the situation for bipolar cell retinal ribbons (Maxeiner et al., 2016), suggests that some features of the IHC ribbon-type $A Z$ can be very well replaced by a ribbonless multi-AZ morphology: the synaptic complement of $\mathrm{Ca}^{2+}$-channels and $\mathrm{SVs}$, as well as exocytic $\Delta \mathrm{C}_{\mathrm{m}}$ elicited by strong depolarizations, were similar. Therefore, we likely underestimated the role of the ribbon in sound encoding in our present study.

For sound encoding at the afferent synapses between IHCs and SGNs, we observed some commonalities and differences with the bassoon mutants and $\mathrm{RBE}^{\mathrm{KO} / \mathrm{KO}}$ mice, whereby the stronger phenotype of bassoon mutants suggests additive effects of bassoon and ribbon loss. Recordings from single SGNs indicate reduced peak and adapted release rates at the IHC synapses of ribbonless synapses, as well as impaired temporal precision of coding. High temporal precision is a hallmark of synaptic sound encoding (e.g. (Köppl, 1997). Reduced release rates or smaller EPSC sizes would increase the temporal jitter (Buran et al., 2010; Li et al., 2014; Rutherford et al., 2012; Wittig and Parsons, 2008). Reduced spike rates and increased jitter of release likely explain the reduced ABR wave I amplitude in both mutants. A striking difference from bassoon mutants, however, is that sound encoding in $\mathrm{RBE}^{\mathrm{KO} / \mathrm{KO}}$ mice was impaired substantially, despite unaltered exocytic $\Delta \mathrm{C}_{\mathrm{m}}$ upon strong stimulation. We propose two mechanisms with likely additive effects to explain this surprising finding: i) the small depolarized shift of synaptic $\mathrm{Ca}^{2+}$-channels might contribute the lower spontaneous and evoked firing rates as well as higher sound thresholds of SGNs and ii) the reduced SVreplenishment might not suffice to balance the rate of consumption leading to a smaller standing RRP in vivo, while the arrest of exocytosis in the voltage-clamped IHCs for tens of seconds likely enables complete filling of the release sites (max. standing RRP). The changes in $\mathrm{Ca}^{2+}$-channel gating observed in IHCs of RBE ${ }^{\mathrm{KO} / K O}$ mice were unexpected, as so far, a direct or indirect interaction of RIBEYE and $\mathrm{Ca}^{2+}$-channels have not been described. Clearly, future studies, including studies on the potential regulation of $\mathrm{Ca}_{\mathrm{v}} 1.3 \mathrm{Ca}^{2+}$-channels by RIBEYE and piccolino in heterologous expression systems, paired pre- and postsynaptic recordings, as well as further computational modeling, are required. 


\section{Materials and methods}

\section{Key resources table}

\begin{tabular}{|c|c|c|c|c|}
\hline $\begin{array}{l}\text { Reagent type (species) } \\
\text { or resource }\end{array}$ & Designation & Source or reference & Identifiers & Additional information \\
\hline $\begin{array}{l}\text { Strain, strain background } \\
\text { (Mus musculus) }\end{array}$ & $\begin{array}{l}\text { Constitutive RIBEYE knockout, } \\
\text { C57BL/6 background }\end{array}$ & PMID: 26929012 & & \\
\hline Antibody & CtBP2 (mouse monoclonal) & BD Biosciences & 612044 & $1: 200$ \\
\hline Antibody & PSD-95 (mouse monoclonal) & Sigma Aldrich & P246-100ul & $1: 200$ \\
\hline Antibody & $\begin{array}{l}\text { Bassoon SAP7f407 } \\
\text { (mouse monoclonal) }\end{array}$ & Abcam & ab82958 & $1: 200$ \\
\hline Antibody & Bassoon (guinea pig polyclonal) & Synaptic Systems & 141004 & $1: 500$ \\
\hline Antibody & RIM2 (rabbit polyclonal) & Synaptic Systems & 140103 & $1: 100$ \\
\hline Antibody & Cav1.3 (rabbit polyclonal) & Alomone Labs & ACC 005 & $\begin{array}{l}1: 75 \\
\text { or } 1: 100\end{array}$ \\
\hline Antibody & Piccolino (rabbit polyclonal) & Regus-Leidig et al. (2013) & & $1: 500$ \\
\hline Antibody & $\begin{array}{l}\text { Parvalbumin } \alpha \\
\text { (guinea pig polyclonal) }\end{array}$ & Synaptic Systems & 195004 & $1: 1000$ \\
\hline Antibody & $\begin{array}{l}\text { Calbindin } 28 \text { k } \\
\text { (mouse monoclonal) }\end{array}$ & Swant & $07(F)$ & $1: 500$ \\
\hline Antibody & Calretinin (rabbit polyclonal) & Swant & 1893-0114 & $1: 1000$ \\
\hline Antibody & $\begin{array}{l}\text { Alexa Fluor } 488 \text { conjugated } \\
\text { anti-rabbit (goat polyclonal) }\end{array}$ & Invitrogen & A11008 & $1: 200$ \\
\hline Antibody & $\begin{array}{l}\text { Alexa Fluor } 488 \text { conjugated } \\
\text { anti-guinea-pig (goat polyclonal) }\end{array}$ & Invitrogen & A11004 & $1: 200$ \\
\hline Antibody & $\begin{array}{l}\text { Alexa Fluor } 568 \text { conjugated } \\
\text { anti-mouse (goat polyclonal) }\end{array}$ & Invitrogen & A11073 & $1: 200$ \\
\hline Antibody & $\begin{array}{l}\text { Alexa Fluor } 647 \text { conjugated } \\
\text { anti-rabbit (donkey polyclonal) }\end{array}$ & Invitrogen & A31573 & $1: 200$ \\
\hline Antibody & $\begin{array}{l}\text { STAR580 conjugated } \\
\text { anti-mouse (goat polyclonal) }\end{array}$ & Abberior & 2-0002-005-1 & $1: 200$ \\
\hline Antibody & $\begin{array}{l}\text { STAR580 conjugated } \\
\text { anti-rabbit (goat polyclonal) }\end{array}$ & Abberior & 2-0012-005-8 & $1: 200$ \\
\hline Antibody & $\begin{array}{l}\text { STAR635p conjugated } \\
\text { anti-mouse (goat polyclonal) }\end{array}$ & Abberior & 2-0002-007-5 & $1: 200$ \\
\hline Antibody & $\begin{array}{l}\text { STAR635p conjugated } \\
\text { anti-rabbit (goat polyclonal) }\end{array}$ & Abberior & 2-0012-007-2 & $1: 200$ \\
\hline Software, algorithm & Patchmaster or Pulse & $\begin{array}{l}\text { http://www.heka.com/products/ } \\
\text { products_main.html\#soft_pm }\end{array}$ & RRID:SCR_000034 & \\
\hline Software, algorithm & IGOR Pro & $\begin{array}{l}\text { http://www.wavemetrics.com/products } \\
\text { /igorpro/igorpro.htm }\end{array}$ & RRID:SCR_000325 & \\
\hline Software, algorithm & Patchers Power Tools & $\begin{array}{l}\text { http://www3.mpibpc.mpg.de/groups } \\
\text { /neher/index.php?page=software }\end{array}$ & RRID:SCR_001950 & \\
\hline Software, algorithm & MATLAB & $\begin{array}{l}\text { http://www.mathworks.com/ } \\
\text { products/matlab/ }\end{array}$ & RRID:SCR_001622 & \\
\hline Software, algorithm & Gatan Microscopy Suite & $\begin{array}{l}\text { http://www.gatan.com/products/tem } \\
\text {-analysis/gatan-microscopy-suite-software }\end{array}$ & RRID:SCR_014492 & $\begin{array}{l}\text { DigitalMicrograph } \\
\text { scripting }\end{array}$ \\
\hline Software, algorithm & Reconstruct & PMID: 15817063 & & \\
\hline Software, algorithm & Serial-EM & PMID: 16182563 & & \\
\hline Software, algorithm & $3 d m o d$ & PMID: 8742726 & & \\
\hline Software, algorithm & IMOD & http://bio3d.colorado.edu/imod & RRID:SCR_003297 & \\
\hline Software, algorithm & Genetic fit algorithm & Sanchez del Rio and Pareschi, 2001 & doi: $10.1117 / 12.411624$ & \\
\hline Software, algorithm & Fiji & http://fiji.sc & RRID:SCR_002285 & \\
\hline
\end{tabular}

Continued on next page 
Continued

\begin{tabular}{llll}
$\begin{array}{l}\text { Reagent type (species) } \\
\text { or resource }\end{array}$ & Designation & Source or reference & Identifiers \\
\hline Software, algorithm & ImageJ & https://imagej.nih.gov/ij/ & RRID:SCR_003070 \\
\hline Software, algorithm & Imaris & http://www.bitplane.com/imaris/imaris & RRID:SCR_007370 \\
\hline Software, algorithm & Excel & microsoft.com/mac/excel & \\
\hline Software, algorithm & Origin & $\begin{array}{l}\text { http://www.originlab.com/index. } \\
\text { aspx?go=PRODUCTS/Origin }\end{array}$ & RRID:SCR_014212 \\
\hline Software, algorithm & GraphPad Prism & $\begin{array}{l}\text { https://www.graphpad.com/ } \\
\text { scientific-software/prism/ }\end{array}$ & RRID:SCR_015807 \\
\hline Software, algorithm & Java Statistical Classes library & Bertie, 2002 & \\
\hline
\end{tabular}

\section{Animals}

Constitutive RIBEYE knockout mice (RBE ${ }^{\mathrm{KO} / \mathrm{KO}}$ derived from Ctbp2 $2^{\text {tm } 1.2 S u d}$ by Cre-recombination) were generated by Maxeiner and colleagues (Maxeiner et al., 2016) and were back-crossed to C57BL/6 for five generations (corresponding to a C57BL/6 background contribution of $>95 \%$ ). All experiments complied with national animal care guidelines and were approved by the University of Göttingen Board for Animal Welfare and the Animal Welfare Office of the State of Lower Saxony (permit number: 14-1391). The colony was maintained by mating heterozygous mice. Whenever possible, experiments were performed in parallel on mutant mice and their wildtype littermates. However, the experimental schedule did not always permit this and we occasionally used individual mice from the same colony but without littermate controls. Moreover, for some experiments giving rise to Figure 6-figure supplement 1 and Figure 7E,F,G,H we also used C57BI/6 wild-type mice and combined their results with those of wildtype littermate controls.

\section{Patch-clamp and confocal $\mathrm{Ca}^{2+}$-imaging}

The apical 2/3 turns of organs of Corti from P14 to P28 aged mice were freshly dissected in HEPES Hank's solution containing (in mM): $5.36 \mathrm{KCl}, 141.7 \mathrm{NaCl}, 10 \mathrm{HEPES}, 0.5 \mathrm{MgSO}_{4} 7 \mathrm{H}_{2} \mathrm{O}, 1 \mathrm{MgCl}_{2^{-}}$ $6 \mathrm{H}_{2} \mathrm{O}, 5.6 \mathrm{D}$-glucose, and $3.4 \mathrm{~L}$-glutamine $(\mathrm{pH} \mathrm{7.2,}-300 \mathrm{mOsm})$. The basolateral membranes of the IHCs were exposed by carefully removing the surrounding cells with a suction pipette. All experiments were conducted at room temperature $\left(20-25^{\circ} \mathrm{C}\right)$.

Perforated-patch-clamp recordings were performed as described previously (Moser and Beutner, 2000). The pipette solution contained (in $\mathrm{mM}$ ): $130 \mathrm{Cs}$-gluconate, 10 tetraethylammonium (TEA)-Cl, 10 4-AP, $10 \mathrm{HEPES}, 1 \mathrm{MgCl}_{2}$, as well as $300 \mu \mathrm{g} / \mathrm{ml}$ amphotericin $\mathrm{B}$ (pH 7.2, 280 mOsm). The extracellular solution contained the following (in mM): $112 \mathrm{NaCl}, 35 \mathrm{TEA}-\mathrm{Cl}, 2.8 \mathrm{KCl}, 1 \mathrm{MgCl}, 1 \mathrm{CsCl}, 10$ HEPES, $2 \mathrm{CaCl}_{2}$, and $11.1 \mathrm{D}$-glucose $(\mathrm{pH} \mathrm{7.2,} \mathrm{305} \mathrm{mOsm).} \mathrm{External} \mathrm{solution} \mathrm{change} \mathrm{was} \mathrm{done} \mathrm{by}$ bath exchange through a perfusion system.

For live-imaging, the patch pipette solution contained (in $\mathrm{mM}$ ): $111 \mathrm{Cs}$-glutamate, $1 \mathrm{MgCl}_{2}, 1$ $\mathrm{CaCl}_{2}, 10$ EGTA, 13 TEA-Cl, 20 HEPES, 4 Mg-ATP, $0.3 \mathrm{Na}-\mathrm{GTP}$ and 1 L-Glutathione (pH 7.3, 290 mOsm). To visualize the $\mathrm{Ca}^{2+}$-hotspots and the ribbons, the $\mathrm{Ca}^{2+}$-indicator Fluo-4FF penta- $\mathrm{K}^{+}$salt (0.8 mM, Life Technologies, Germany) and the TAMRA-conjugated CtBP2/RIBEYE-binding dimer peptide (10 $\mu \mathrm{M}$, Biosynthan, Germany) were added to the intracellular solution. The extracellular solution contained the following (in $\mathrm{mM}$ ): $2.8 \mathrm{KCl}, 102 \mathrm{NaCl}, 10 \mathrm{HEPES}, 1 \mathrm{CsCl}_{2}, 1 \mathrm{MgCl}_{2}, 5 \mathrm{CaCl}_{2}$, 35 TEA-Cl, and 11.1 D-Glucose (pH 7.2, 300 mOsm).

EPC-10 amplifiers controlled by Patchmaster or Pulse software (HEKA Elektronik, Germany) were used for the measurements. IHCs were held at $-87 \mathrm{mV}$ or $-69 \mathrm{mV}$. All voltages were corrected for liquid junction potential offline $(17 \mathrm{mV}$ or $14 \mathrm{mV}$, depending on intra- and extracellular solutions used) and voltage-drops across the series resistance $\left(R_{s}\right)$. Currents were leak corrected using a $p / 10$ protocol in exocytosis experiments. Recordings were discarded when the leak current exceeded -55 pA, $R_{\mathrm{s}}$ exceeded $30 \mathrm{M} \Omega$ (for perforated-patch) or $15 \mathrm{M} \Omega$ within 4 min after break-in (for ruptured-patch), or $\mathrm{Ca}^{2+}$-current rundown exceeded $25 \%$. All passive electrical properties of the patchclamp recording experiments are detailed in Supplementary file 2.

Exocytosis was studied by measuring the membrane capacitance increments $\left(\Delta C_{m}\right)$ using the Lindau-Neher technique (Lindau and Neher, 1988). Cells were stimulated by step depolarizations of 
different durations to -17 or $-14 \mathrm{mV}$, or by $100 \mathrm{~ms}$ pulses to voltages ranging from -53 to -37 $\mathrm{mV}$. A resting interval of 10-100 s between the stimuli was used. Each protocol was applied two to three times and only IHCs with reproducible exocytosis during the rounds were included. For display, traces were subjected to 1, 5 or 10 pass Binomial Smoothing using Igor Pro. Current-voltage relationships ('IVs') displayed in Figure 5A (ruptured-patch, 3-week-old mice) were obtained by applying $20 \mathrm{~ms}$ depolarizing step pulses of increasing voltage from -82 to $63 \mathrm{mV}$ in $5 \mathrm{mV}$ steps.

$\mathrm{Ca}^{2+}$-imaging was performed with a spinning disk confocal scanner (CSU22, Yokogawa, Germany) mounted on an upright microscope (Axio Examiner, Zeiss, Germany) with 63x, 1.0 NA objective (W Plan-Apochromat, Zeiss). Images were acquired by a scientific CMOS camera (Neo, Andor, Germany). $\mathrm{Ca}^{2+}$-indicator F4FF and TAMRA-conjugated peptide were excited by diodepumped solid-state lasers with $491 \mathrm{~nm}$ and $561 \mathrm{~nm}$ wavelength, respectively (Cobolt AB). The spinning disk was set to $2000 \mathrm{rpm}$ to synchronize with the $10 \mathrm{~ms}$ acquisition time of the camera.

Using a piezo positioner for the objective (Piezosystem, Germany), a scan of the entire cell was performed $4 \mathrm{~min}$ after breaking into the cell, taking sections each $0.5 \mu \mathrm{m}$ at an exposure time of 0.5 $s$ in the red (TAMRA-peptide) channel from the bottom to the top of the cell. In order to study the voltage-dependence of $\mathrm{Ca}^{2+}$-indicator fluorescence increments at the synapses, the confocal scans were acquired every $0.5 \mu \mathrm{m}$ from the bottom to the top ribbon in the RBE ${ }^{W T} / \mathrm{WT}$ mice. For the $\mathrm{RBE}^{\mathrm{KO} / \mathrm{KO}}$ mice, the scanning was done from the bottom of the cell to $+12 \mu \mathrm{m}$, which on average corresponds to the bottom of the nucleus. $\mathrm{Ca}^{2+}$-currents were evoked by applying a voltage ramp stimulus from -87 to $+63 \mathrm{mV}$ during $150 \mathrm{~ms}(1 \mathrm{mV} / \mathrm{ms})$ in each focal plane. Simultaneously, fluorescence measurements were made in the green channel (Fluo-4FF) with a frame rate of $100 \mathrm{~Hz}$. In order to overcome the limitations of the frame rate and increase the voltage resolution of the fluorescent signal acquired, the voltage ramp protocol was applied twice, once shifted by $5 \mathrm{~ms}$ such that for any given frame during the second ramp the voltage was shifted by $5 \mathrm{mV}$ compared to the first stimulus. Alternating planes were acquired to avoid photobleaching encountered with the consecutive plane acquisition.

\section{Immunohistochemistry, confocal and high resolution STED imaging}

Apical turns of organs of Corti from 3-week-old mice were prepared for 'whole-mount imaging' as described in (Ohn et al., 2016). In brief, the samples were fixed either in formaldehyde (4\%, $10 \mathrm{~min}$ on ice), or methanol $\left(20 \mathrm{~min}\right.$ at $\left.-20^{\circ} \mathrm{C}\right)$. Afterwards, the following primary antibodies were used: mouse anti-CtBP2 (1:200, BD Biosciences, Germany 612044), mouse anti-PSD-95 (1:200, Sigma Aldrich, Germany P246-100ul), mouse anti-bassoon SAP7f407 (1:200, Abcam, Germany, ab82958), guinea pig anti-bassoon (1:500, Synaptic Systems, Germany, 141 004), rabbit anti-RIM2 (1:100, Synaptic Systems 140 103), rabbit anti-Ca 1.3 (1:75 or 1:100, Alomone Labs, Germany, ACC 005), rabbit anti-piccolino (1:500, kind gift of JH Brandstätter; see Regus-Leidig et al., 2013), guinea pig antiparvalbumin $\alpha$ (1:1000, Synaptic Systems, 195 004), mouse anti-calbindin 28 k (1:500, Swant, Germany, 07(F)), and rabbit anti-calretinin (1:1000, Swant 1893-0114). Secondary antibodies used were Alexa Fluor 488 conjugated anti-rabbit, Alexa Fluor 488 conjugated anti-guinea-pig, Alexa Fluor 568 conjugated anti-mouse, and Alexa Fluor 647 conjugated anti-rabbit (1:200, Invitrogen, Germany, A11008, A11004, A11073, and A31573 respectively). For high resolution STED microscopy, STAR580 and STAR635p conjugated anti-rabbit and anti-mouse (1:200, Abberior, Germany, 2-0002-005-1, 2-0012-005-8, 2-0002-007-5, and 2-0012-007-2) have been used as secondary antibodies. Images were acquired using either a Leica SP5 with a 1.4 NA 63x oil immersion objective or an Abberior Instruments Expert Line STED microscope, with excitation lasers at 488, 561, and $633 \mathrm{~nm}$ and STED lasers at $595 \mathrm{~nm}, 1 \mathrm{~W}$, and $775 \mathrm{~nm}, 1.2 \mathrm{~W}$, using a 1.4 NA 100x oil immersion objective, either in confocal or in 2D-STED mode. Images were adjusted for brightness and contrast using ImageJ.

\section{Systems physiology: Auditory Brainstem Responses (ABR), Distortion Product Otoacoustic Emissions (DPOAE) and extracellular recordings from SGNs}

ABR, DPOAE and extracellular recordings from single SGNs were performed essentially as described before (Jing et alı, 2013; Strenzke et al., 2016). ABR and DPOAE recordings were performed on 6week-old mice. For extracellular recordings from individual SGNs, 6 to 10 week-old mice were 
anesthetized by i.p. injection of urethane $(1.32 \mathrm{mg} / \mathrm{kg})$, xylazine $(5 \mathrm{mg} / \mathrm{kg})$ and buprenorphine $(0.1$ $\mathrm{mg} / \mathrm{kg}$ ), a tracheostomy was performed and the mice were then placed in a stereotactic system. After partial removal of the occipital bone and cerebellum to expose the anteroventral cochlear nucleus (AVCN), a glass microelectrode was advanced through the posterior AVCN portion to reach the auditory nerve. Acoustic stimulation was provided by an open field Avisoft ScanSpeak Ultrasonic Speaker (Avisoft Bioacoustics, Germany), and 'putative' SGNs (auditory nerve fibers formed by the central SGN axons) were identified and distinguished from cochlear nucleus neurons based on their stereotactic position (>1.1 mm from the surface of the cochlear nucleus), spontaneous and noiseburst induced firing, peristimulus time histogram (PSTH), regularity of firing, and first spike latency. Recordings were performed using TDT system III hardware and an ELC-03XS amplifier (NPI Electronics, Germany), offline analysis using waveform-based spike detection using custom-written MATLAB software (Source code 1).

\section{Transmission electron microscopy}

Conventional embedding

Conventional embedding of organs of Corti was essentially performed as described previously (Wong et al., 2014). In brief, here P21, 6 weeks and 8 months old mice were used. The apical turn of organs of Corti were dissected in phosphate-buffer saline (PBS) and fixed for $1 \mathrm{hr}$ on ice with $4 \%$ paraformaldehyde and $0.5 \%$ glutaraldehyde in PBS $(\mathrm{pH} 7.4)$. After an additional fixation overnight on ice with $2 \%$ glutaraldehyde in $0.1 \mathrm{M}$ sodium cacodylate buffer $(\mathrm{pH} 7.2)$, samples were washed in $0.1 \mathrm{M}$ sodium cacodylate buffer and placed in $1 \%$ osmium tetroxide $(\mathrm{v} / \mathrm{v}$ in $0.1 \mathrm{M}$ sodium cacodylate buffer) on ice for $1 \mathrm{hr}$. Next, samples were washed twice in $0.1 \mathrm{M}$ sodium cacodylate buffer (10 min each, on ice) and further in distilled water and subsequently en bloc stained with $1 \%$ uranyl acetate ( $v / v$ in distilled water) for $1 \mathrm{hr}$ on ice. Uranyl acetate treated samples were briefly washed three times in distilled water, dehydrated using a series of increasing ethanol concentration and finally embedded in epoxy resin (Agar $100 \mathrm{kit}$, Plano, Germany) and polymerized for $48 \mathrm{hr}$ at $70^{\circ} \mathrm{C}$. An Ultracut $\mathrm{E}$ microtome (Leica Microsystems, Germany) equipped with a $35^{\circ}$ diamond knife (Diatome, Switzerland) was used to obtain ultrathin sections $(70-75 \mathrm{~nm})$ of the specimen. Sections were transferred to $1 \%$ formvar-coated ( $\mathrm{w} / \mathrm{v}$ in water-free chloroform) copper slot grids (ATHENE copper slot grids, 3.05 $\mathrm{mm} \varnothing, 1 \mathrm{~mm} \times 2 \mathrm{~mm}$; Plano, Germany) and subsequently stained with uranyl acetate replacement solution (UAR-EMS) (Science Services, Germany) and Reynold's lead citrate. The specimens were investigated at $80 \mathrm{kV}$ with a JEM1011 transmission electron microscope (JEOL, Germany) and micrographs acquired at 10,000-x magnification using a Gatan Orius 1200A camera (Gatan, Germany, using the Digital Micrograph software package). Serial 3D reconstructions of ultrathin sections were generated with the program Reconstruct (Fiala, 2005).

\section{High-pressure freezing/freeze-substitution (HPF/FS) and electron tomography}

High-pressure freezing, freeze-substitution followed by electron tomography were essentially performed as described previously (Vogl et al., 2015; Jung et al., 2015a). After freeze-substitution and embedding in epoxy resin (Agar $100 \mathrm{kit}$, Plano, Germany), $250 \mathrm{~nm}$ semithin sections for electron tomography were obtained on an Ultracut E ultramicrotome (Leica Microsystems, Germany) with a $35^{\circ}$ diamond knife (Diatome, Switzerland). Sections were placed on $1 \%$ formvar-coated $(\mathrm{w} / \mathrm{v}$ in water-free chloroform) copper 100 mesh grids (ATHENE, Plano, Germany, $3.05 \mathrm{~mm} \varnothing$ ) and poststained with UAR-EMS (Science Services, Germany) and Reynold's lead citrate.

For electron tomography, $10 \mathrm{~nm}$ gold particles (British Bio Cell/Plano, Germany) were applied to both sides of the stained grids. Single tilt series at 12,000-x magnification, mainly from -60 to $+60^{\circ}$ (if only fewer angles were possible, the tomograms were only accepted for quantification if the quality was sufficient) were acquired with an $1^{\circ}$ increment at a JEM2100 (JEOL, Germany)) transmission electron microscope at $200 \mathrm{kV}$ using the Serial-EM software (Mastronarde, 2005). The tomograms were generated using the IMOD package etomo and models were generated using $3 \mathrm{dmod}$ (Kremer et al., 1996). 


\section{Data analysis}

Live-imaging and IHC-patch-clamp data were analyzed using custom programs in lgor Pro 6.3 (Wavemetrics, Portland, OR, USA; Source Code 2). For analysis of IV-curves, the evoked $\mathrm{Ca}^{2+}$-current was averaged from 5 to $10 \mathrm{~ms}$ after the start of the depolarization. The total $\mathrm{Ca}^{2+}$-charge was estimated by taking the integral of the leak-subtracted current during the depolarization step. For most protocols, $\Delta C_{m}$ was estimated as the difference between the mean of $C_{m} 400 \mathrm{~ms}$ after and before the depolarization (the initial $60 \mathrm{~ms}$ after the end of depolarization were skipped). For paired pulse experiments, the calculation of the mean of $C_{m}$ before and after the depolarization was limited to the time remaining in the inter pulse interval after skipping (the initial $30 \mathrm{~ms}$ after the end of depolarization were skipped).

$\Delta \mathrm{F}$ images were generated by subtracting the fluorescence intensity inside the cell at the resting state $\left(F_{0}\right.$, an average of 10 frames) from the one at the depolarized state (an average of 6 frames during voltage ramp protocol). $\Delta \mathrm{F}$ was calculated as the average of a $3 \times 3$ pixel square placed in the region showing the greatest intensity increase within the fluorescence hotspot. Maximal $\Delta F$ $\left(\Delta \mathrm{F}_{\text {max }}\right)$ was the average of $5 \Delta \mathrm{F}$ values obtained between -17 and $+8 \mathrm{mV}$ during the voltage ramp (around the peak $\mathrm{Ca}^{2+}$-influx). Only $\mathrm{AZs}$ presenting a $\Delta \mathrm{F}_{\max }$ greater than the mean of the fluorescence intensity plus two standard deviations at rest were considered for further analysis. For analysis of the voltage dependence of synaptic $\mathrm{Ca}^{2+}$-signals, raw traces were fitted to the following

$$
F(V)=F_{0}+\frac{f_{v} \cdot\left(V_{r}-V\right)}{1+e^{\frac{\left(V_{h}-V\right)}{k}}}
$$

where $f_{v}$ is the fluorescence-voltage-relationship $\Delta \mathrm{F} / \Delta \mathrm{V}$ obtained by linear fitting to the FV-curve in the range of 3 to $23 \mathrm{mV}, V_{r}$ the reversal potential of $65.6 \mathrm{mV}$, and $V$ the command voltage, in order to obtain $V_{h}$, the voltage of half-maximal activation, and $k$, the slope factor. The spatial extent of the synaptic $\mathrm{Ca}^{2+}$-signals was estimated by fitting of a 2D Gaussian function to the fluorescent hotspot using a genetic fit algorithm (Sanchez del Rio and Pareschi, 2001) to obtain the full width at half maximum in the long and short axis. For each spot, the calculations were made at those confocal sections where the intensity of the spot was strongest.

Activation time constants of $\mathrm{Ca}^{2+}$-currents at differing potentials were obtained by fitting to the first $5 \mathrm{~ms}$ of the current traces the following equation:

$$
f(t)=y_{0}+A \times\left(1-e^{\left(\frac{-x}{\tau}\right)}\right)^{2}
$$

Confocal and STED immunofluorescence images were analyzed and z-projected with Fiji software and further analyzed using Igor Pro. For synapse counting, co-localized pre- and postsynaptic immunofluorescent spots were counted manually. The spatial extent of the line-shaped $\mathrm{Ca}^{2+}$-channel clusters was estimated by fitting a 2D Gaussian function to the individual clusters in 2D STED images to obtain the full width at half maximum in the long and short axis. The areas of the PSD were calculated by the following formula: area $=\pi \times$ (Long Axis/2) (Short Axis/2). The semi-quantitative immunofluorescence analysis of the proteinaceous $\mathrm{Ca}^{2+}$-buffers was performed by calculating the mean immunofluorescence intensity of a volume $(40(\mathrm{X}) \times 40(\mathrm{Y}) \times 4(\mathrm{Z})$ voxels or $2.8 \times 2.8 \times 2 \mu \mathrm{m})$ below the nucleus and above the synapses. This and the count and intensity of the CtBP2 immunofluorescent spots have been analyzed in Imaris 7.6.5 with custom Matlab routines (Source Code 4).

For extracellular SGN recordings, PSTHs were calculated as average firing rates across 200 presentations of $50 \mathrm{~ms}$ or $500 \mathrm{~ms}$ tone bursts presented at $0.1 \mathrm{~s} / 0.2 \mathrm{~s}$ or $2 \mathrm{~s}$ intervals, resp. (PSTH at 10/ $5 \mathrm{~Hz}$ and $0.5 \mathrm{~Hz}$ ) at $\mathrm{C}_{\mathrm{f}}, 30 \mathrm{~dB}$ above the threshold and binned at a width of $2 \mathrm{~ms}$. Peak rate was determined as the largest bin of the PSTH in a time window 3-11 ms after stimulus onset. Adapted rate was averaged in a window spanning $35-45 \mathrm{~ms}$ or $405-415 \mathrm{~ms}$ after stimulus onset (for PSTH at $5 \mathrm{~Hz}$ and $0.5 \mathrm{~Hz}$, respectively). Rate level functions were acquired using $50 \mathrm{~ms}$ tone bursts presented at $\mathrm{C}_{\mathrm{f}}$ and at $5 \mathrm{~Hz}$. 25 repetitions for each stimulus intensity (5 dB steps) were recorded. Maximal steepness was calculated as the maximal increase in spike rate between two consecutive $5 \mathrm{~dB}$ increment steps. Dynamic range was calculated by using sigmoidal fits in the rate level functions as described in and measuring the range of sound pressure between $10 \%$ and $90 \%$ of maximal firing rate. For amplitude modulation analysis, synchronization index was calculated as described by 
(Goldberg and Brown, 1969). Synchronization index estimation was only considered valid when at least 15 spikes occurred in a $3 \mathrm{~s}$ time window and the Rayleigh statistic was below 13.8.

For analysis of forward masking experiments, spike counts in a $10 \mathrm{~ms}$ interval starting from responses of both masker and probe onset were determined and presented as the ratio of probe and masker responses for at least 25 repetitions for every masker-probe interval from each unit. Exponential fitting to the plots of each individual SGN approximated the recovery kinetics.

Quantitative analysis of electron microscopy data was performed with ImageJ for conventional embedded samples and with IMOD for HPF/FS tomograms. According to the presence of ribbonoccupied and ribbonless synapses, we considered the following analysis criteria:

For ribbon-occupied synapses, membrane-proximal synaptic vesicles (MP-SVs, within a distance of $\leq 25 \mathrm{~nm}$ from the $A Z$ membrane and $\leq 80 \mathrm{~nm}$ from the presynaptic density) and ribbon-associated synaptic vesicles (RA-SVs, first layer around the ribbon with a maximum distance of $80 \mathrm{~nm}$ from the vesicle membrane to the ribbon) were counted (Figure $2 \mathrm{~J}$, random sections analysis according to Strenzke et al., 2016 and Figure 3G for tomograms according to 2D-random section analysis criteria). The tomogram analysis parameters were further modified, as used in Jung et al., 2015a. Here, the MP-SVs were defined as vesicles with $\leq 50 \mathrm{~nm}$ from the AZ membrane and with the shortest distance from the vesicle membrane to the presynaptic density of $\leq 100 \mathrm{~nm}$, excluding RA-SVs (Figure 3-figure supplement 1A). For random sections, SV diameters were calculated by the averaged measurements of the horizontal and vertical axis. The ribbon size was measured in height and width, taking the longest axis of the ribbon excluding the PD, and the edges of the synaptic ribbon were traced manually using ImageJ. The length of the PD was measured along the AZ membrane (Figure 2J).

For ribbonless synapses, a presynaptic density-associated synaptic vesicle (PDA-SVs) pool was defined considering all clustered vesicles $\leq 80 \mathrm{~nm}$ around the PD that did not fulfill the criteria of a MP-SV (see above, also Figure $2 \mathrm{~J}$ for random sections). The MP-SV pool, as well as the SV diameter and PD length, were analyzed as for the ribbon-occupied synapses. For tomograms, the PDA-SV pool was defined as the SVs in the first layer $\leq 80 \mathrm{~nm}$ to the PD, excluding the MP-SVs. The MP-SV pool criteria are the same as described in the previous paragraph (Figure 3G, according to 2D-random section and Figure 3-figure supplement 1A, according to Jung et al., 2015a). For tomograms, the according pools were further distinguished into tethered and non-tethered vesicles (Figure 3G and Figure 3-figure supplement 1A). All vesicles were annotated using a spherical 'scattered object' at its maximum projection in the tomogram, encompassing the outer leaflet of the vesicles. The vesicle radii were determined automatically (Helmprobst et al., 2015) with the program 'imodinfo option - $p$ ' of the IMOD software package (Kremer et al., 1996).

\section{Release site model of RRP release and replenishment}

The coding of sound onset differs among the various SGNs in time due to different durations of the traveling wave, synaptic delays and conduction times. To obtain an average PSTH for modeling that is not smeared out due to such differences between units, the individual PSTHs were aligned beforehand by shifting their timing relative to each other. Onset detection was based on a change in spike statistics. For spontaneous activity, the 99.5 percentile of spike counts was determined. Next, the time at which response rises to twice this percentile was found. This is certainly a point within the sound response. Finally going back from this point, a drop back baseline activity, that is below the percentile was detected and used as onset time. Aligned PSTH from all units were averaged. This averaged PSTH from the forward masking data were fit with a model waveform using a genetic fit algorithm implemented in IGOR Pro (Wavemetrics, Lake Oswego, OR, USA). The purpose of the model is to give insight into the dynamics of SV cycling at the average IHC AZ. More specifically, the notion of $\mathrm{Ca}^{2+}$-nanodomain-like control of RRP exocytosis (Brandt et al., 2005; Graydon et al., 2011; Pangršic et alo, 2015; Wong et alo, 2014), as well as the limited MP-SVs at the AZ (see Figures 2 and 3) motivates the notion of a limited, quasi-fixed number of available vesicular release sites or slots, $\mathrm{N}_{\text {slot }}($ Frank et al., 2010; Wong et al., 2014) that constitute the RRP. Each of these sites can be either empty or occupied by a release ready SV (whereby all filled slots constitute the 'standing' RRP) and at each time point, a release ready SV will fuse with a certain probability described by the fusion rate constant $k_{\text {fus. }}$. Its value depends on the sound pressure level in a relation we assume to be linear within the dynamic range of the synapse/fiber. While the sound pressure level rises from silence to saturation $k_{\text {fus }}$ increases from $k_{\text {fus, spont }}$ to $k_{\text {fus,stim. }}$. The refilling of empty 
sites is described by a refill rate constant $k_{\text {refill, }}$ which also depends on the sound intensity $\left(k_{\text {refill, spont }}\right.$ to $\mathrm{k}_{\text {refill,stim }}$ ).

The state of the release site was described by:

$$
\frac{d N_{\text {slot }}^{\text {filled }}(t)}{d t}=k_{\text {refill }}(t) N_{\text {slot }}-N_{\text {slot }}^{\text {filled }}(t)\left(k_{\text {fus }}(t)+k_{\text {refill }}(t)\right)
$$

Although this equation is formulated for SV fusion rates, a scaling factor $f$ can be used to account for the fraction of fusion events that cannot successfully trigger an action potential (AP) despite sufficient neural excitability for example because of the too small size of the elicited excitatory post synaptic current. This factor effectively operates as if the number of release sites was scaled down. The scaled equation then gives a rate R of potentially supra-threshold EPSCs as the product of the number of occupied release sites, the fusion rate constant and the scaling factor $f$ :

$$
R(t)=k_{\text {fus }}(t) . f \cdot N_{\text {slot }}^{\text {filled }}(t)
$$

The stationary solutions of Equation 3 together with Equation 4 determine steady state occupancy and steady state event rates:

$$
\begin{gathered}
N_{\text {solt }}^{\text {filled }} \mid \begin{array}{l}
\text { condition } \\
\text { stradystate }
\end{array}=\frac{K_{\text {refill }}^{\text {condition }}}{K_{\text {fusion }}^{\text {condition }}+K_{\text {refill }}^{\text {condition }}} \cdot N_{\text {solt }} \\
\left.R\right|_{\mid \begin{array}{l}
\text { stradystate } \\
\text { condition }
\end{array}}=\frac{K_{\text {fusion }}^{\text {condition }} \cdot f \cdot K_{\text {refill }}^{\text {condition }}}{K_{\text {fusion }}^{\text {condition }}+K_{\text {refill }}^{\text {condion }}} \cdot N_{\text {solt }}
\end{gathered}
$$

In this equation, 'condition' is either silence or saturating sound pressure level.

In order to connect the postsynaptic event rate of potentially supra-threshold EPSCs to the actual AP rate, refractoriness is considered as a combination of an absolute refractory period $t_{a b s,}$ during which the probability of an EPSC to trigger an AP is zero, with a relative refractory period during which this trigger probability returns to one with an exponential time course characterized by $\tau_{\text {rel }}$ (Berry and Meister, 1998). This description of refractoriness can be applied to the 'driving' EPSC rate $\mathrm{R}$ by means of a delayed differential equation. The equation is motivated by the concept of three possible states of the SGN: 'absolute refractory', 'relative refractory' or 'available' (fully excitable). At any point, the probability that the SGN turns from 'available' to 'refractory' is proportional to the rate $\mathrm{R}(\mathrm{t})$. The return back to 'available' happens 'delayed' by $\mathrm{t}_{\mathrm{abs}}$ and with a probability that is proportional to $1 / \tau_{\text {rel }}$.

$$
\begin{gathered}
\frac{d f_{\text {avail }}(t)}{d t}=\frac{f_{\text {relref }}(t)}{\tau_{\text {rel }}}-f_{\text {avail }}(t) \cdot f \cdot R(t) \\
\frac{d f_{\text {relref }}(t)}{d t}=f_{\text {avail }}\left(t-t_{\text {abs }}\right) \cdot f \cdot R\left(t-t_{\text {abs }}\right)-\frac{f_{\text {relref }}(t)}{\tau_{\text {rel }}}
\end{gathered}
$$

Together with Equation 6 the stationary solution of this description of refractoriness connects the observable steady state rates during silence and stimulation to the rate constants $k_{\text {refill }}$ und $k_{\text {fusion }}$ :

$$
\text { AP_Rate }\left.\right|_{\text {strady state }} ^{\text {condition }}=\frac{K_{\text {fusion }}^{\text {condition }} \cdot f \cdot K_{\text {refill }}^{\text {condion }}}{K_{\text {fusion }}^{\text {condition }}+K_{\text {refill }}^{\text {condion }}} \cdot N_{\text {solt }} \cdot \frac{1}{1+R \mid \begin{array}{l}
\text { condition } \\
\text { stradystate }
\end{array}\left(t_{\text {abs }}+\tau_{\text {rel }}\right)}
$$

To go beyond the description of steady state event rates and to use the model for a parameterized description of the actual time course of experimentally observed PSTHs acquired during forward masking (Figure 11E), it is necessary to define the relation between the applied stimulus and the fusion and refill rate constants. For the experimental data presented here, the stimulus level was increased from silence to $30 \mathrm{~dB}$ above fiber threshold within a $4 \mathrm{~ms}$ ramp having a quarter of a $\sin ^{2}$ shape. It was assumed that $k_{\text {fusion }}$ and $k_{\text {refill }}$ follow the stimulus increase simultaneously. The ordinary differential and delayed differential equations above were combined into a fit function. PSTHs (one masker followed by one probe) were averaged per genotype for each masker probe interval $(4,16$, 64 and $256 \mathrm{~ms}$ ) and were fitted in parallel with one parameter set. During experiments, trials were acquired in immediate succession without gaps. Therefore, the model implements cyclic boundary conditions for the occupancy of the slots. This model only captures the short term processes, 
assuming that a set of experiments, for example forward masking trials quickly lead to a steady state. Slow adapting processes were not explicitly modeled. The observed drop of the apparent number of available slots in the forward masking experiments was described here as a change in the number of slots from $\mathrm{N}_{\text {slots }}$ to a reduced capacity $\mathrm{N}^{\prime}$ slots and for a given spiral ganglion neuron that was tested with tone bursts and forward masking, the ratio $\mathrm{N}_{\text {slots }} / \mathrm{N}^{\prime}$ slots could be estimated from the change in rates (see Results).

\section{Statistical analysis}

The data were analyzed using Matlab (Mathworks), Excel, Igor Pro 6 (Wavemetrics), Origin 9.0 (Microcal Software), and GraphPad Prism (GraphPad Software). Averages were expressed as mean \pm standard error of the mean (S.E.M.). For every dataset, the standard deviation (S.D.), number of replicates $(n)$ and animals $(N)$ were indicated. For Figure $7, n_{\min }$ corresponds to the minimum number of cells included in the analysis of each depolarization potential given that the number of cells for each potential differs. In order to compare two samples, data sets were tested for normal distribution (Jarque-Bera test, D'Agostino and Pearson omnibus normality test or the Shapiro-Wilk test) and equality of variances (F-test), followed by two-tailed unpaired Student's t-test, or, when data were not normally distributed and/or variance was unequal between samples, the unpaired two-tailed Mann-Whitney-Wilcoxon test was used. Cumulative distributions in Figure 9A were statistically compared using the Kolmogorov-Smirnov test. The ROUT method ( $\mathrm{Q}=0.1 \%$ ) from GraphPad Prism was used to identify definitive outliers for Figure $7 \mathrm{H}$.

For multiple comparisons, statistical significance was calculated by using one-way ANOVA test (two-way ANOVA in the case of ABR thresholds) followed by Tukey's test for normally distributed data or Kruskal-Wallis (K-W) test followed by non-parametric multiple comparisons test (NPMC) for non-normally distributed data.

For SV diameter quantifications in random sections, a custom-written routine using Java Statistical Classes library (JSC) (Bertie, 2002) was utilized for statistical analysis (Source code 5). Due to the tied ranks of SV diameter measurements obtained for random sections, their S.E.M. was used as a tolerance value for the usage of Kruskal-Wallis test as suggested by Bertie et al. in JSC library (Bertie, 2002), where two values were treated as equal if their difference was $\leq$ S.E.M.. The non-significant difference between samples is reported as n.s., significant differences are reported as ${ }^{*} p<0.05$, ${ }^{\star *} \mathrm{p}<0.01,{ }^{\star * *} \mathrm{p}<0.001,{ }^{\star * \star *} \mathrm{p}<0.0001$.

\section{Acknowledgements}

We thank N Dietrich, T Goldak, S Gerke, C Senger-Freitag and I Herfort for expert technical assistance, Gerhard Hoch for software and analysis development, and Frank Schmitz for providing $\mathrm{RBE}^{\mathrm{KO} / \mathrm{WT}}$ mice.

\section{Additional information}

Funding

\begin{tabular}{lll} 
Funder & Grant reference number & Author \\
\hline $\begin{array}{l}\text { Deutsche Forschungsge- } \\
\text { meinschaft }\end{array}$ & $\begin{array}{l}\text { Collaborative Research } \\
\text { Center 889, projects A2, A6, Nicola Strenzk } \\
\text { and A7 }\end{array}$ & $\begin{array}{l}\text { Carolin Wichmann } \\
\text { Tobias Moser }\end{array}$ \\
\hline $\begin{array}{l}\text { Deutsche Forschungsge- } \\
\text { meinschaft }\end{array}$ & $\begin{array}{l}\text { Tobias Moser Leibniz } \\
\text { program MO 896/51 }\end{array}$ & $\begin{array}{l}\text { Tobias Moser } \\
\text { Max-Planck-Gesellschaft }\end{array}$ \\
\hline Max-Planck-Fellowship & Tobias Moser \\
\hline Niedersächsisches Vorab & & Tobias Moser
\end{tabular}

The funders had no role in study design, data collection and interpretation, or the decision to submit the work for publication. 
Author contributions

Philippe Jean, Data curation, Formal analysis, Investigation, Visualization, Writing-original draft, Writing-review and editing, PJ performed immunohistochemistry and confocal immunofluorescence microscopy, patch-clamp, Ca2+ imaging, computational modeling and prepared the manuscript; David Lopez de la Morena, Data curation, Formal analysis, Investigation, Visualization, Writing-original draft, Writing-review and editing, DL performed in vivo extracellular recordings from single SGNs and AVCN neurons, and contributed to the manuscript; Susann Michanski, Data curation, Formal analysis, Investigation, Visualization, Writing—original draft, Writing-review and editing, SM performed conventional embedding and electron microscopy of random and serial sections, contributed to high-pressure freezing and to the manuscript; Lina María Jaime Tobón, Data curation, Formal analysis, Investigation, Visualization, Writing-original draft, Writing-review and editing, LMJT performed patch-clamp capacitance measurements and contributed to the manuscript; Rituparna Chakrabarti, Data curation, Formal analysis, Investigation, Visualization, Writing—original draft, Writing-review and editing, RC performed high-pressure freezing, freeze-substitution and electron tomography, and contributed to the manuscript; Maria Magdalena Picher, Data curation, Formal analysis, Investigation, Visualization, Writing-original draft, MMP performed patch-clamp capacitance measurements and contributed to the manuscript; Jakob Neef, Data curation, Software, Formal analysis, Supervision, Investigation, Visualization, Methodology, Writing-original draft, JN performed immunohistochemistry, confocal and STED immunofluorescence microscopy, co-supervised PJ and LMJT, and contributed to the manuscript; SangYong Jung, Data curation, Formal analysis, Investigation, SYJ performed immunohistochemistry and confocal immunofluorescence microscopy; Mehmet Gültas, Software, Formal analysis, Methodology, MG contributed a statistical analysis of electron microscopy data; Stephan Maxeiner, Resources, Writing-original draft, SM contributed mutant mice and genetic expertise; Andreas Neef, Conceptualization, Software, Formal analysis, Supervision, Methodology, Writing-original draft, AN designed the study, performed and supervised computational modeling, and contributed to the manuscript; Carolin Wichmann, Conceptualization, Supervision, Visualization, Writing-original draft, Writing-review and editing, CW designed the study, supervised electron microscopy and tomography, and contributed to the manuscript; Nicola Strenzke, Conceptualization, Software, Supervision, Methodology, NS designed the study and supervised in vivo extracellular recordings from single SGNs; Chad Grabner, Conceptualization, Writing-original draft, CG designed the study and contributed to the manuscript; Tobias Moser, Conceptualization, Resources, Supervision, Funding acquisition, Validation, Visualization, Methodology, Writing-original draft, Project administration, Writing-review and editing, TM designed the study, prepared the manuscript and co-supervised PJ, DL and LMJT

\section{Author ORCIDs}

Philippe Jean (iD) http://orcid.org/0000-0001-5325-1370

David Lopez de la Morena (iD) http://orcid.org/0000-0003-0835-2732

Susann Michanski (D) https://orcid.org/0000-0001-5893-1981

Lina María Jaime Tobón (iD http://orcid.org/0000-0002-6752-7750

Maria Magdalena Picher (D) https://orcid.org/0000-0003-0722-3883

Jakob Neef (iD) http://orcid.org/0000-0002-4757-9385

Andreas Neef (iD) https://orcid.org/0000-0003-4445-7478

Carolin Wichmann (iD http://orcid.org/0000-0001-8868-8716

Tobias Moser (iD) http://orcid.org/0000-0001-7145-0533

Ethics

Animal experimentation: All experiments complied with national animal care guidelines and were approved by the University of Göttingen Board for Animal Welfare and the Animal Welfare Office of the State of Lower Saxony (permit number: 14-1391).

Decision letter and Author response

Decision letter https://doi.org/10.7554/eLife.29275.031

Author response https://doi.org/10.7554/eLife.29275.032 


\section{Additional files}

Supplementary files

- Source code 1. Matlab scripts for the waveform-based spike detection.

DOI: https://doi.org/10.7554/eLife.29275.022

- Source code 2. Live calcium imaging analysis.

DOI: https://doi.org/10.7554/eLife.29275.023

- Source Code 3. Routines for the analysis of whole-cell capacitance measurements

DOI: https://doi.org/10.7554/eLife.29275.024

- Source Code 4. Matlab scripts for the count andintensity of immunofluorescent spots.

DOI: https://doi.org/10.7554/eLife.29275.025

- Source code 5. Java routine for the SV diameter quantifications in random sections

DOI: https://doi.org/10.7554/eLife.29275.026

- Supplementary file 1. Modified tomogram analysis. The table depicts the modified tomogram analysis performed according to Jung et al., 2015a displaying the mean \pm (S.E.M.), (S.D.), p-values, sample size and statistical tests used to compare $\mathrm{RBE}^{\mathrm{WT} / \mathrm{WT}}$ and $\mathrm{RBE}^{\mathrm{KO} / \mathrm{KO}}$. Refer to Figure 3-figure supplement 1 for the graphs.

DOI: https://doi.org/10.7554/eLife.29275.027

- Supplementary file 2. Passive electrical properties of patch-clamp recording experiments.

The table shows the mean \pm (S.E.M.) of the passive electrical properties across all ruptured and perforated patch-clamp recording experiments in $\mathrm{RBE}^{\mathrm{WT} / \mathrm{WT}}$ and $\mathrm{RBE}^{\mathrm{KO} / \mathrm{KO}}$ conditions.

DOI: https://doi.org/10.7554/eLife.29275.028

- Transparent reporting form

DOI: https://doi.org/10.7554/eLife.29275.029

\section{References}

Bartoletti TM, Jackman SL, Babai N, Mercer AJ, Kramer RH, Thoreson WB. 2011. Release from the cone ribbon synapse under bright light conditions can be controlled by the opening of only a few $\mathrm{Ca}(2+)$ channels. Journal of Neurophysiology 106:2922-2935. DOI: https://doi.org/10.1152/jn.00634.2011, PMID: 21880934

Berry MJ, Meister M., 1998. Refractoriness and neural precision. Journal of Neuroscience 18:2200-2211. PMID: 9482804

Bertie A. 2002. Java applications for teaching statistics. MSOR Connections 2:78-81. DOI: https://doi.org/10. 11120/msor.2002.02030078

Brandt A, Khimich D, Moser T. 2005. Few CaV1.3 channels regulate the exocytosis of a synaptic vesicle at the hair cell ribbon synapse. Journal of Neuroscience 25:11577-11585. DOI: https://doi.org/10.1523/JNEUROSCI. 3411-05.2005, PMID: 16354915

Brandt A, Striessnig J, Moser T. 2003. CaV1.3 channels are essential for development and presynaptic activity of cochlear inner hair cells. Journal of Neuroscience 23:10832-10840. PMID: 14645476

Bunt AH. 1971. Enzymatic digestion of synaptic ribbons in amphibian retinal photoreceptors. Brain Research 25: 571-577. DOI: https://doi.org/10.1016/0006-8993(71)90461-6, PMID: 5544325

Buran BN, Strenzke N, Neef A, Gundelfinger ED, Moser T, Liberman MC. 2010. Onset coding is degraded in auditory nerve fibers from mutant mice lacking synaptic ribbons. Journal of Neuroscience 30:7587-7597. DOI: https://doi.org/10.1523/JNEUROSCI.0389-10.2010, PMID: 20519533

Cole AA, Chen X, Reese TS. 2016. A Network of three types of filaments organizes synaptic vesicles for storage, mobilization, and docking. Journal of Neuroscience 36:3222-3230. DOI: https://doi.org/10.1523/JNEUROSCl. 2939-15.2016, PMID: 26985032

Cui G, Meyer AC, Calin-Jageman I, Neef J, Haeseleer F, Moser T, Lee A. 2007. Ca2+-binding proteins tune Ca2 +-feedback to Cav1.3 channels in mouse auditory hair cells. The Journal of physiology 585:791-803. DOI: https://doi.org/10.1113/jphysiol.2007.142307, PMID: 17947313

Davydova D, Marini C, King C, Klueva J, Bischof F, Romorini S, Montenegro-Venegas C, Heine M, Schneider R, Schröder MS, Altrock WD, Henneberger C, Rusakov DA, Gundelfinger ED, Fejtova A. 2014. Bassoon specifically controls presynaptic P/Q-type $\mathrm{Ca}(2+)$ channels via RIM-binding protein. Neuron 82:181-194. DOI: https://doi.org/10.1016/j.neuron.2014.02.012, PMID: 24698275

Dick O, Hack I, Altrock WD, Garner CC, Gundelfinger ED, Brandstätter JH. 2001. Localization of the presynaptic cytomatrix protein piccolo at ribbon and conventional synapses in the rat retina: comparison with Bassoon. Journal of Comparative Neurology 439:224-234. DOI: https://doi.org/10.1002/cne.1344, PMID: 11596050 
Dick O, tom Dieck S, Altrock WD, Ammermüller J, Weiler R, Garner CC, Gundelfinger ED, Brandstätter JH. 2003. The presynaptic active zone protein bassoon is essential for photoreceptor ribbon synapse formation in the retina. Neuron 37:775-786. DOI: https://doi.org/10.1016/S0896-6273(03)00086-2, PMID: 12628168

Dou H, Vazquez AE, Namkung Y, Chu H, Cardell EL, Nie L, Parson S, Shin HS, Yamoah EN. 2004. Null mutation of alpha1D Ca2+ channel gene results in deafness but no vestibular defect in mice. Journal of the Association for Research in Otolaryngology : JARO 5:215-226. DOI: https://doi.org/10.1007/s10162-003-4020-3, PMID: 15357422

Fernández-Busnadiego R, Asano S, Oprisoreanu AM, Sakata E, Doengi M, Kochovski Z, Zürner M, Stein V, Schoch S, Baumeister W, Lucić V. 2013. Cryo-electron tomography reveals a critical role of RIM1 $\alpha$ in synaptic vesicle tethering. The Journal of Cell Biology 201:725-740. DOl: https://doi.org/10.1083/jcb.201206063, PMID: 23712261

Fiala JC. 2005. Reconstruct: a free editor for serial section microscopy. Journal of Microscopy 218:52-61. DOI: https://doi.org/10.1111/j.1365-2818.2005.01466.x, PMID: 15817063

Frank T, Khimich D, Neef A, Moser T. 2009. Mechanisms contributing to synaptic Ca2+ signals and their heterogeneity in hair cells. PNAS 106:4483-4488. DOI: https://doi.org/10.1073/pnas.0813213106, PMID: 1 9246382

Frank T, Rutherford MA, Strenzke N, Neef A, Pangršič T, Khimich D, Fejtova A, Fetjova A, Gundelfinger ED, Liberman MC, Harke B, Bryan KE, Lee A, Egner A, Riedel D, Moser T. 2010. Bassoon and the synaptic ribbon organize $\mathrm{Ca}^{2}+$ channels and vesicles to add release sites and promote refilling. Neuron 68:724-738. DOI: https://doi.org/10.1016/j.neuron.2010.10.027, PMID: 21092861

Goldberg JM, Brown PB. 1969. Response of binaural neurons of dog superior olivary complex to dichotic tonal stimuli: some physiological mechanisms of sound localization. Journal of Neurophysiology 32:613-636. DOI: https://doi.org/10.1152/jn.1969.32.4.613, PMID: 5810617

Grabner CP, Zenisek D. 2013. Amperometric resolution of a prespike stammer and evoked phases of fast release from retinal bipolar cells. Journal of Neuroscience 33:8144-8158. DOI: https://doi.org/10.1523/JNEUROSCI. 5062-12.2013, PMID: 23658155

Graydon CW, Cho S, Li GL, Kachar B, von Gersdorff H, Gersdorff Hvon. 2011. Sharp Ca ${ }^{2+}$ nanodomains beneath the ribbon promote highly synchronous multivesicular release at hair cell synapses. Journal of Neuroscience 31: 16637-16650. DOI: https://doi.org/10.1523/JNEUROSCI.1866-11.2011, PMID: 22090491

Graydon CW, Zhang J, Oesch NW, Sousa AA, Leapman RD, Diamond JS. 2014. Passive diffusion as a mechanism underlying ribbon synapse vesicle release and resupply. Journal of Neuroscience 34:8948-8962. DOI: https:// doi.org/10.1523/JNEUROSCI.1022-14.2014, PMID: 24990916

Hallermann S, Fejtova A, Schmidt H, Weyhersmüller A, Silver RA, Gundelfinger ED, Eilers J. 2010. Bassoon speeds vesicle reloading at a central excitatory synapse. Neuron 68:710-723. DOI: https://doi.org/10.1016/j. neuron.2010.10.026, PMID: 21092860

Harris DM, Dallos P. 1979. Forward masking of auditory nerve fiber responses. Journal of Neurophysiology 42 : 1083-1107. DOI: https://doi.org/10.1152/jn.1979.42.4.1083, PMID: 479921

Heil P, Neubauer H, Irvine DR, Brown M. 2007. Spontaneous activity of auditory-nerve fibers: insights into stochastic processes at ribbon synapses. Journal of Neuroscience 27:8457-8474. DOI: https://doi.org/10.1523/ JNEUROSCI.1512-07.2007, PMID: 17670993

Helmprobst F, Frank M, Stigloher C. 2015. Presynaptic architecture of the larval zebrafish neuromuscular junction. Journal of Comparative Neurology 523:1984-1997. DOI: https://doi.org/10.1002/cne.23775, PMID: 25766140

Hibino H, Pironkova R, Onwumere O, Vologodskaia M, Hudspeth AJ, Lesage F. 2002. RIM binding proteins (RBPs) couple Rab3-interacting molecules (RIMs) to voltage-gated $\mathrm{Ca}(2+)$ channels. Neuron 34:411-423. DOI: https://doi.org/10.1016/S0896-6273(02)00667-0, PMID: 11988172

Hildebrand JD, Soriano P. 2002. Overlapping and unique roles for C-terminal binding protein 1 (CtBP1) and CtBP2 during mouse development. Molecular and Cellular Biology 22:5296-5307. DOI: https://doi.org/10. 1128/MCB.22.15.5296-5307.2002, PMID: 12101226

Huang LC, Barclay M, Lee K, Peter S, Housley GD, Thorne PR, Montgomery JM. 2012. Synaptic profiles during neurite extension, refinement and retraction in the developing cochlea. Neural Development 7:38-17. DOI: https://doi.org/10.1186/1749-8104-7-38, PMID: 23217150

Hull C, Studholme K, Yazulla S, von Gersdorff H. 2006. Diurnal changes in exocytosis and the number of synaptic ribbons at active zones of an ON-type bipolar cell terminal. Journal of Neurophysiology 96:2025-2033. DOI: https://doi.org/10.1152/jn.00364.2006, PMID: 16738212

Jackman SL, Choi SY, Thoreson WB, Rabl K, Bartoletti TM, Kramer RH. 2009. Role of the synaptic ribbon in transmitting the cone light response. Nature Neuroscience 12:303-310. DOI: https://doi.org/10.1038/nn.2267, PMID: 19219039

Jarsky T, Tian M, Singer JH. 2010. Nanodomain control of exocytosis is responsible for the signaling capability of a retinal ribbon synapse. Journal of Neuroscience 30:11885-11895. DOI: https://doi.org/10.1523/JNEUROSCI. 1415-10.2010, PMID: 20826653

Jing Z, Rutherford MA, Takago H, Frank T, Fejtova A, Khimich D, Moser T, Strenzke N. 2013. Disruption of the presynaptic cytomatrix protein bassoon degrades ribbon anchorage, multiquantal release, and sound encoding at the hair cell afferent synapse. Journal of Neuroscience 33:4456-4467. DOI: https://doi.org/10.1523/ JNEUROSCI.3491-12.2013, PMID: 23467361 
Johnson SL, Olt J, Cho S, von Gersdorff H, Marcotti W. 2017. The Coupling between Ca2+ channels and the exocytotic $\mathrm{Ca} 2+$ sensor at hair cell ribbon synapses varies tonotopically along the mature cochlea. The Journal of Neuroscience 37:2471-2484. DOI: https://doi.org/10.1523/JNEUROSCI.2867-16.2017, PMID: 28154149

Joris PX, Carney LH, Smith PH, Yin TC. 1994. Enhancement of neural synchronization in the anteroventral cochlear nucleus. I. Responses to tones at the characteristic frequency. Journal of Neurophysiology 71:10221036. DOI: https://doi.org/10.1152/jn.1994.71.3.1022, PMID: 8201399

Jung S, Maritzen T, Wichmann C, Jing Z, Neef A, Revelo NH, Al-Moyed H, Meese S, Wojcik SM, Panou I, Bulut H, Schu P, Ficner R, Reisinger E, Rizzoli SO, Neef J, Strenzke N, Haucke V, Moser T. 2015b. Disruption of adaptor protein $2 \mu(\mathrm{AP}-2 \mu)$ in cochlear hair cells impairs vesicle reloading of synaptic release sites and hearing. The EMBO Journal 34:2686-2702. DOI: https://doi.org/10.15252/embj.201591885, PMID: 26446278

Jung S, Oshima-Takago T, Chakrabarti R, Wong AB, Jing Z, Yamanbaeva G, Picher MM, Wojcik SM, Göttfert F, Predoehl F, Michel K, Hell SW, Schoch S, Strenzke N, Wichmann C, Moser T. 2015a. Rab3-interacting molecules $2 \alpha$ and $2 \beta$ promote the abundance of voltage-gated $\mathrm{CaV} 1.3 \mathrm{Ca} 2+$ channels at hair cell active zones. PNAS 112:E3141-E3149. DOI: https://doi.org/10.1073/pnas.1417207112, PMID: 26034270

Khimich D, Nouvian R, Pujol R, Tom Dieck S, Egner A, Gundelfinger ED, Moser T. 2005. Hair cell synaptic ribbons are essential for synchronous auditory signalling. Nature 434:889-894. DOI: https://doi.org/10.1038/ nature03418, PMID: 15829963

Kremer JR, Mastronarde DN, McIntosh JR. 1996. Computer visualization of three-dimensional image data using IMOD. Journal of Structural Biology 116:71-76. DOI: https://doi.org/10.1006/jsbi.1996.0013, PMID: 8742726

Krinner S, Butola T, Jung S, Wichmann C, Moser T. 2017. RIM-binding protein 2 promotes a large number of $\mathrm{CaV} 1.3 \mathrm{Ca} 2+$-channels and contributes to fast synaptic vesicle replenishment at hair cell active zones. Frontiers in Cellular Neuroscience 11:334. DOI: https://doi.org/10.3389/fncel.2017.00334, PMID: 29163046

Köppl C. 1997. Phase locking to high frequencies in the auditory nerve and cochlear nucleus magnocellularis of the barn owl, Tyto alba. Journal of Neuroscience 17:3312. PMID: 9096164

Lagnado L, Schmitz F. 2015. Ribbon synapses and visual processing in the retina. Annual Review of Vision Science 1:235-262. DOI: https://doi.org/10.1146/annurev-vision-082114-035709, PMID: 28532378

Lee A, Wong ST, Gallagher D, Li B, Storm DR, Scheuer T, Catterall WA. 1999. Ca2+/calmodulin binds to and modulates P/Q-type calcium channels. Nature 399:155-159. DOI: https://doi.org/10.1038/20194, PMID: 10335 845

Lenzi D, Crum J, Ellisman MH, Roberts WM. 2002. Depolarization redistributes synaptic membrane and creates a gradient of vesicles on the synaptic body at a ribbon synapse. Neuron 36:649-659. DOI: https://doi.org/10. 1016/S0896-6273(02)01025-5, PMID: 12441054

Li GL, Cho S, von Gersdorff H. 2014. Phase-locking precision is enhanced by multiquantal release at an auditory hair cell ribbon synapse. Neuron 83:1404-1417. DOI: https://doi.org/10.1016/j.neuron.2014.08.027, PMID: 251 99707

Liberman MC. 1978. Auditory-nerve response from cats raised in a low-noise chamber. The Journal of the Acoustical Society of America 63:442-455. DOI: https://doi.org/10.1121/1.381736, PMID: 670542

Limbach C, Laue MM, Wang X, Hu B, Thiede N, Hultqvist G, Kilimann MW. 2011. Molecular in situ topology of Aczonin/Piccolo and associated proteins at the mammalian neurotransmitter release site. PNAS 108:E392E401. DOI: https://doi.org/10.1073/pnas.1101707108, PMID: 21712437

Lindau M, Neher E. 1988. Patch-clamp techniques for time-resolved capacitance measurements in single cells. Pflugers Archiv European Journal of Physiology 411:137-146. DOI: https://doi.org/10.1007/BF00582306, PMID: 3357753

Lv C, Stewart WJ, Akanyeti O, Frederick C, Zhu J, Santos-Sacchi J, Sheets L, Liao JC, Zenisek D. 2016. Synaptic ribbons require ribeye for electron density, proper synaptic localization, and recruitment of calcium channels. Cell Reports 15:2784-2795. DOI: https://doi.org/10.1016/j.celrep.2016.05.045, PMID: 27292637

Magupalli VG, Schwarz K, Alpadi K, Natarajan S, Seigel GM, Schmitz F. 2008. Multiple RIBEYE-RIBEYE interactions create a dynamic scaffold for the formation of synaptic ribbons. Journal of Neuroscience 28:79547967. DOI: https://doi.org/10.1523/JNEUROSCI.1964-08.2008, PMID: 18685021

Mastronarde DN. 2005. Automated electron microscope tomography using robust prediction of specimen movements. Journal of Structural Biology 152:36-51. DOI: https://doi.org/10.1016/j.jsb.2005.07.007, PMID: 16182563

Maxeiner S, Luo F, Tan A, Schmitz F, Südhof TC. 2016. How to make a synaptic ribbon: RIBEYE deletion abolishes ribbons in retinal synapses and disrupts neurotransmitter release. The EMBO Journal 35:1098-1114. DOI: https://doi.org/10.15252/embj.201592701, PMID: 26929012

Mehta B, Snellman J, Chen S, Li W, Zenisek D. 2013. Synaptic ribbons influence the size and frequency of miniature-like evoked postsynaptic currents. Neuron 77:516-527. DOI: https://doi.org/10.1016/j.neuron.2012. 11.024, PMID: 23395377

Mendoza Schulz A, Jing Z, Sánchez Caro JM, Wetzel F, Dresbach T, Strenzke N, Wichmann C, Moser T. 2014. Bassoon-disruption slows vesicle replenishment and induces homeostatic plasticity at a CNS synapse. The EMBO Journal 33:n/a-527. DOI: https://doi.org/10.1002/embj.201385887, PMID: 24442636

Moser T, Beutner D. 2000. Kinetics of exocytosis and endocytosis at the cochlear inner hair cell afferent synapse of the mouse. PNAS 97:883-888. DOI: https://doi.org/10.1073/pnas.97.2.883, PMID: 10639174

Moser T, Vogl C. 2016. New insights into cochlear sound encoding. F1000Research 5:2081. DOl: https://doi.org/ 10.12688/f1000research.8924.1 
Neef J, Urban NT, Ohn T-L, Frank T, Jean P, Hell SW, Willig KI, Moser T. 2018. Quantitative optical nanophysiology of $\mathrm{Ca} 2+$ signaling at inner hair cell active zones. Nature Communications 9. DOI: https://doi. org/10.1038/s41467-017-02612-y

Oesch NW, Diamond JS. 2011. Ribbon synapses compute temporal contrast and encode luminance in retinal rod bipolar cells. Nature Neuroscience 14:1555-1561. DOI: https://doi.org/10.1038/nn.2945, PMID: 22019730

Ohn TL, Rutherford MA, Jing Z, Jung S, Duque-Afonso CJ, Hoch G, Picher MM, Scharinger A, Strenzke N, Moser T. 2016. Hair cells use active zones with different voltage dependence of $\mathrm{Ca} 2+$ influx to decompose sounds into complementary neural codes. PNAS 113:E4716-E4725. DOI: https://doi.org/10.1073/pnas.1605737113, PMID: 27462107

Pangršič T, Gabrielaitis M, Michanski S, Schwaller B, Wolf F, Strenzke N, Moser T. 2015. EF-hand protein Ca2+ buffers regulate $\mathrm{Ca} 2+$ influx and exocytosis in sensory hair cells. PNAS 112:E1028-E1037. DOI: https://doi.org/ 10.1073/pnas.1416424112, PMID: 25691754

Pangršič T, Reisinger E, Moser T. 2012. Otoferlin: a multi-C2 domain protein essential for hearing. Trends in Neurosciences 35:671-680. DOI: https://doi.org/10.1016/j.tins.2012.08.002, PMID: 22959777

Peterson BZ, DeMaria CD, Adelman JP, Yue DT. 1999. Calmodulin is the Ca2+ sensor for Ca2+-dependent inactivation of L-type calcium channels. Neuron 22:549-558. DOI: https://doi.org/10.1016/S0896-6273(00) 80709-6, PMID: 10197534

Picher MM, Gehrt A, Meese S, Ivanovic A, Predoehl F, Jung S, Schrauwen I, Dragonetti AG, Colombo R, Van Camp G, Strenzke N, Moser T, Camp GV. 2017. Ca2+-binding protein 2 inhibits Ca2+-channel inactivation in mouse inner hair cells. PNAS 114:E1717-E1726. DOI: https://doi.org/10.1073/pnas.1617533114, PMID: 281837 97

Platzer J, Engel J, Schrott-Fischer A, Stephan K, Bova S, Chen H, Zheng H, Striessnig J. 2000. Congenital deafness and sinoatrial node dysfunction in mice lacking class D L-type Ca2+ channels. Cell 102:89-97. DOI: https://doi.org/10.1016/S0092-8674(00)00013-1, PMID: 10929716

Regus-Leidig H, Ott C, Löhner M, Atorf J, Fuchs M, Sedmak T, Kremers J, Fejtová A, Gundelfinger ED, Brandstätter JH. 2013. Identification and immunocytochemical characterization of Piccolino, a novel Piccolo splice variant selectively expressed at sensory ribbon synapses of the eye and ear. PLoS One 8:e70373. DOI: https://doi.org/10.1371/journal.pone.0070373, PMID: 23936420

Robertson D, Paki B. 2002. Role of L-type Ca2+ channels in transmitter release from mammalian inner hair cells. II. Single-neuron activity. Journal of Neurophysiology 87:2734-2740. DOI: https://doi.org/10.1152/jn.2002.87.6. 2734, PMID: 12037175

Russell IJ, Sellick PM. 1983. Low-frequency characteristics of intracellularly recorded receptor potentials in guinea-pig cochlear hair cells. The Journal of Physiology 338:179-206. DOI: https://doi.org/10.1113/jphysiol. 1983.sp014668, PMID: 6875955

Rutherford MA, Chapochnikov NM, Moser T. 2012. Spike encoding of neurotransmitter release timing by spiral ganglion neurons of the cochlea. Journal of Neuroscience 32:4773-4789. DOI: https://doi.org/10.1523/ JNEUROSCI.4511-11.2012, PMID: 22492033

Rutherford MA, Moser T. 2016. The Ribbon Synapse Between Type I Spiral Ganglion Neurons and Inner Hair Cells. In: Dabdoub A, Fritzsch B, Popper A. N, Fay R. R (Eds). The Primary Auditory Neurons of the Mammalian Cochlea. New York: Springer. p. 117-156. DOI: https://doi.org/10.1007/978-1-4939-3031-9_5

Safieddine S, El-Amraoui A, Petit C. 2012. The auditory hair cell ribbon synapse: from assembly to function. Annual Review of Neuroscience 35:509-528. DOI: https://doi.org/10.1146/annurev-neuro-061010-113705, PMID: 22715884

Sanchez del Rio M, Pareschi G. 2001. Global optimization and reflectivity data fitting for x-ray multilayer mirrors by means of genetic algorithms. Advances in X-Ray Optics 88-96. DOI: https://doi.org/10.1117/12.411624

Schmitz F, Königstorfer A, Südhof TC. 2000. RIBEYE, a component of synaptic ribbons: a protein's journey through evolution provides insight into synaptic ribbon function. Neuron 28:857-872. DOI: https://doi.org/10. 1016/S0896-6273(00)00159-8, PMID: 11163272

Schrauwen I, Helfmann S, Inagaki A, Predoehl F, Tabatabaiefar MA, Picher MM, Sommen M, Zazo Seco C, Oostrik J, Kremer H, Dheedene A, Claes C, Fransen E, Chaleshtori MH, Coucke P, Lee A, Moser T, Van Camp G. 2012. A mutation in CABP2, expressed in cochlear hair cells, causes autosomal-recessive hearing impairment. The American Journal of Human Genetics 91:636-645. DOI: https://doi.org/10.1016/j.ajhg.2012. 08.018, PMID: 22981119

Schwarz K, Natarajan S, Kassas N, Vitale N, Schmitz F. 2011. The synaptic ribbon is a site of phosphatidic acid generation in ribbon synapses. Journal of Neuroscience 31:15996-16011. DOI: https://doi.org/10.1523/ JNEUROSCI.2965-11.2011, PMID: 22049442

Sendin G, Bulankina AV, Riedel D, Moser T. 2007. Maturation of ribbon synapses in hair cells is driven by thyroid hormone. Journal of Neuroscience 27:3163-3173. DOI: https://doi.org/10.1523/JNEUROSCI.3974-06.2007, PMID: 17376978

Sheets L, Trapani JG, Mo W, Obholzer N, Nicolson T. 2011. Ribeye is required for presynaptic Ca(V)1.3a channel localization and afferent innervation of sensory hair cells. Development 138:1309-1319. DOI: https://doi.org/ 10.1242/dev.059451, PMID: 21350006

Siksou L, Rostaing P, Lechaire JP, Boudier T, Ohtsuka T, Fejtová A, Kao HT, Greengard P, Gundelfinger ED, Triller A, Marty S. 2007. Three-dimensional architecture of presynaptic terminal cytomatrix. Journal of Neuroscience 27:6868-6877. DOI: https://doi.org/10.1523/JNEUROSCI.1773-07.2007, PMID: 17596435 
Sjostrand FS. 1958. Ultrastructure of retinal rod synapses of the guinea pig eye as revealed by three-dimensional reconstructions from serial sections. Journal of Ultrastructure Research 2:122-170. DOI: https://doi.org/10. 1016/S0022-5320(58)90050-9, PMID: 13631744

Smith CA, Sjostrand FS. 1961. Structure of the nerve endings on the external hair cells of the guinea pig cochlea as studied by serial sections. Journal of Ultrastructure Research 5:523-556. DOI: https://doi.org/10.1016/S00225320(61)80025-7, PMID: 13914158

Snellman J, Mehta B, Babai N, Bartoletti TM, Akmentin W, Francis A, Matthews G, Thoreson W, Zenisek D. 2011. Acute destruction of the synaptic ribbon reveals a role for the ribbon in vesicle priming. Nature Neuroscience 14:1135-1141. DOI: https://doi.org/10.1038/nn.2870, PMID: 21785435

Sobkowicz HM, Rose JE, Scott GE, Slapnick SM. 1982. Ribbon synapses in the developing intact and cultured organ of Corti in the mouse. Journal of Neuroscience 2:942-957. PMID: 7097321

Spiwoks-Becker I, Glas M, Lasarzik I, Vollrath L. 2004. Mouse photoreceptor synaptic ribbons lose and regain material in response to illumination changes. European Journal of Neuroscience 19:1559-1571. DOI: https:// doi.org/10.1111/j.1460-9568.2004.03198.x, PMID: 15066152

Strenzke N, Chakrabarti R, Al-Moyed H, Müller A, Hoch G, Pangrsic T, Yamanbaeva G, Lenz C, Pan KT, Auge E, Geiss-Friedlander R, Urlaub H, Brose N, Wichmann C, Reisinger E. 2016. Hair cell synaptic dysfunction, auditory fatigue and thermal sensitivity in otoferlin lle515Thr mutants. The EMBO Journal 35:2519-2535. DOI: https:// doi.org/10.15252/embj.201694564, PMID: 27729456

Strenzke N, Chanda S, Kopp-Scheinpflug C, Khimich D, Reim K, Bulankina AV, Neef A, Wolf F, Brose N, XuFriedman MA, Moser T. 2009. Complexin-l is required for high-fidelity transmission at the endbulb of Held auditory synapse. Journal of Neuroscience 29:7991-8004. DOI: https://doi.org/10.1523/JNEUROSCI.0632-09. 2009, PMID: 19553439

Taberner AM, Liberman MC. 2005. Response properties of single auditory nerve fibers in the mouse. Journal of Neurophysiology 93:557-569. DOI: https://doi.org/10.1152/jn.00574.2004, PMID: 15456804

tom Dieck S, Altrock WD, Kessels MM, Qualmann B, Regus H, Brauner D, Fejtová A, Bracko O, Gundelfinger ED, Brandstätter JH. 2005. Molecular dissection of the photoreceptor ribbon synapse: physical interaction of Bassoon and RIBEYE is essential for the assembly of the ribbon complex. The Journal of cell biology 168:825836. DOI: https://doi.org/10.1083/jcb.200408157, PMID: 15728193

Vaithianathan T, Matthews G. 2014. Visualizing synaptic vesicle turnover and pool refilling driven by calcium nanodomains at presynaptic active zones of ribbon synapses. PNAS 111:8655-8660. DOI: https://doi.org/10 1073/pnas.1323962111, PMID: 24912160

Van Epps HA, Hayashi M, Lucast L, Stearns GW, Hurley JB, De Camilli P, Brockerhoff SE. 2004. The zebrafish nrc mutant reveals a role for the polyphosphoinositide phosphatase synaptojanin 1 in cone photoreceptor ribbon anchoring. Journal of Neuroscience 24:8641-8650. DOI: https://doi.org/10.1523/JNEUROSCI.2892-04.2004, PMID: 15470129

Vogl C, Cooper BH, Neef J, Wojcik SM, Reim K, Reisinger E, Brose N, Rhee JS, Moser T, Wichmann C. 2015. Unconventional molecular regulation of synaptic vesicle replenishment in cochlear inner hair cells. Journal of Cell Science 128:638-644. DOI: https://doi.org/10.1242/jcs.162099, PMID: 25609709

von Gersdorff H, Vardi E, Matthews G, Sterling P. 1996. Evidence that vesicles on the synaptic ribbon of retinal bipolar neurons can be rapidly released. Neuron 16:1221-1227. DOI: https://doi.org/10.1016/S0896-6273(00) 80148-8, PMID: 8663998

Wichmann C, Moser T. 2015. Relating structure and function of inner hair cell ribbon synapses. Cell and Tissue Research 361:95-114. DOI: https://doi.org/10.1007/s00441-014-2102-7, PMID: 25874597

Wittig JH, Parsons TD. 2008. Synaptic ribbon enables temporal precision of hair cell afferent synapse by increasing the number of readily releasable vesicles: a modeling study. Journal of Neurophysiology 100:17241739. DOI: https://doi.org/10.1152/jn.90322.2008, PMID: 18667546

Wong AB, Rutherford MA, Gabrielaitis M, Pangrsic T, Göttfert F, Frank T, Michanski S, Hell S, Wolf F, Wichmann C, Moser T. 2014. Developmental refinement of hair cell synapses tightens the coupling of $\mathrm{Ca} 2+$ influx to exocytosis. The EMBO Journal 33:n/a-264. DOI: https://doi.org/10.1002/embj.201387110, PMID: 24442635

Yang PS, Alseikhan BA, Hiel H, Grant L, Mori MX, Yang W, Fuchs PA, Yue DT. 2006. Switching of Ca2+dependent inactivation of $\mathrm{Ca}(\mathrm{v}) 1.3$ channels by calcium binding proteins of auditory hair cells. Journal of Neuroscience 26:10677-10689. DOI: https://doi.org/10.1523/JNEUROSCI.3236-06.2006, PMID: 17050707

Zampini V, Johnson SL, Franz C, Lawrence ND, Münkner S, Engel J, Knipper M, Magistretti J, Masetto S,

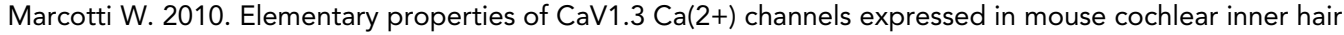
cells. The Journal of Physiology 588:187-199. DOI: https://doi.org/10.1113/jphysiol.2009.181917, PMID: 1 9917569

Zenisek D, Horst NK, Merrifield C, Sterling P, Matthews G. 2004. Visualizing synaptic ribbons in the living cell. Journal of Neuroscience 24:9752-9759. DOI: https://doi.org/10.1523/JNEUROSCI.2886-04.2004, PMID: 15525760 
Endophilin-A regulates presynaptic $\mathrm{Ca} 2+$ influx and synaptic vesicle recycling in auditory hair cells

Jana Kroll*, Lina M Jaime Tobón*, Christian Vogl*, Jakob Neef, Ilona

Kondratiuk, Melanie König, Nicola Strenzke, Carolin Wichmann, Ira Milosevic, Tobias Moser

*These authors contributed equally to the work

EMBO J (2019)38:e100116

DOI: https://doi.org/10.15252/embj.2018100116 


\title{
Endophilin-A regulates presynaptic $\mathrm{Ca}^{2+}$ influx and synaptic vesicle recycling in auditory hair cells
}

\author{
Jana Kroll ${ }^{1,2,3,4, \dagger}$ (D), Lina M Jaime Tobón ${ }^{3,4,5,6,7, \dagger}$ D , Christian Vog $\left.\right|^{3,5,7,8, \dagger}$ (D), Jakob Neef ${ }^{3,5,6,7}$, \\ Ilona Kondratiuk ${ }^{1}$ D) , Melanie König ${ }^{1,3}$, Nicola Strenzke ${ }^{3,7,9}$ (D), Carolin Wichmann 2,3,7, ${ }^{*}$ (D), \\ Ira Milosevic ${ }^{1,3,{ }^{* *}}$ (D) \& Tobias Moser $3,5,6,7,{ }^{* * *}$ (iD
}

\begin{abstract}
Ribbon synapses of cochlear inner hair cells (IHCs) operate with high rates of neurotransmission; yet, the molecular regulation of synaptic vesicle (SV) recycling at these synapses remains poorly understood. Here, we studied the role of endophilins-A1-3, endocytic adaptors with curvature-sensing and curvature-generating properties, in mouse IHCs. Single-cell RT-PCR indicated the expression of endophilins-A1-3 in IHCs, and immunoblotting confirmed the presence of endophilin-A1 and endophilin-A2 in the cochlea. Patch-clamp recordings from endophilin-A-deficient IHCs revealed a reduction of $\mathrm{Ca}^{2+}$ influx and exocytosis, which we attribute to a decreased abundance of presynaptic $\mathrm{Ca}^{2+}$ channels and impaired SV replenishment. Slow endocytic membrane retrieval, thought to reflect clathrin-mediated endocytosis, was impaired. Otoferlin, essential for IHC exocytosis, co-immunoprecipitated with purified endophilin-A1 protein, suggestive of a molecular interaction that might aid exocytosis-endocytosis coupling. Electron microscopy revealed lower SV numbers, but an increased occurrence of coated structures and endosome-like vacuoles at IHC active zones. In summary, endophilins regulate $\mathrm{Ca}^{2+}$ influx and promote SV recycling in IHCs, likely via coupling exocytosis to endocytosis, and contributing to membrane retrieval and SV reformation.
\end{abstract}

Keywords electron microscopy; endocytosis; membrane capacitance; ribbon synapse; super-resolution microscopy

Subject Categories Neuroscience

DOI 10.15252/embj.2018100116 | Received 22 June 2018 | Revised 17 December 2018 | Accepted 20 December 2018 | Published online 7 February 2019

The EMBO Journal (2019) 38: e100116

\section{Introduction}

Ribbon synapses of auditory IHCs faithfully convert acoustic signals into an action potential code in spiral ganglion neurons (SGNs). Individual presynaptic active zones (AZs) of IHCs are thought to drive firing in a single SGN at rates of up to hundreds of $\mathrm{Hz}$ for as long as the sound continues (Safieddine et al, 2012; Wichmann \& Moser, 2015; Rutherford \& Moser, 2016). Exocytosis of synaptic vesicles (SVs) at IHC AZs is both, fast and indefatigable. It seems to operate independently of classical neuronal SNARE proteins, Munc13-like priming factors, or complexins (Strenzke et al, 2009; Nouvian et al, 2011; Vogl et al, 2015), but, instead, involves the deafness gene product otoferlin (Roux et al, 2006; Pangrsic et al, 2010). Probably the most important coordinator of synaptic transmission is $\mathrm{Ca}^{2+}$ that enters IHCs primarily through presynaptic voltage-gated $\mathrm{Ca}_{V} 1.3 \mathrm{Ca}^{2+}$ channels and mediates excitation-secretion coupling (Platzer et al, 2000; Brandt et al, 2003, 2005; Weiler et al, 2014; Wong et al, 2014). At IHC AZs, $\mathrm{Ca}^{2+}$ channels are present in defined numbers, organized in a stripe-like manner, and show little inactivation, which enables reliable information transfer during sustained stimulation (Brandt et al, 2005; Frank et al, 2009, 2010; Ohn et al, 2016; Neef et al, 2018). Interestingly, $\mathrm{Ca}^{2+}$ channels have been reported to interact with otoferlin (Ramakrishnan et al, 2009) and with endophilins (Chen et al, 2003), the protein family under study here.

To sustain high transmission rates, IHCs need to balance exocytosis by equally efficient SV recycling (Siegel \& Brownell, 1986; Parsons et al, 1994; Moser \& Beutner, 2000; Beutner et al, 2001; Lenzi et al, 2002; Trapani et al, 2009; Neef et al, 2014; Revelo et al, 2014; Jung et al, 2015). Here, at least three kinetically distinct forms

1 Synaptic Vesicle Dynamics Group, European Neuroscience Institute (ENI), University Medical Center Göttingen, Göttingen, Germany

2 Molecular Architecture of Synapses Group, Institute for Auditory Neuroscience, InnerEarLab and Center for Biostructural Imaging of Neurodegeneration, University Medical Center Göttingen, Göttingen, Germany

3 Collaborative Research Center 889, University of Göttingen, Göttingen, Germany

4 Göttingen Graduate School for Neuroscience and Molecular Biosciences, University of Göttingen, Göttingen, Germany

5 Institute for Auditory Neuroscience and InnerEarLab, University Medical Center Göttingen, Göttingen, Germany

6 Synaptic Nanophysiology Group, Max Planck Institute for Biophysical Chemistry, Göttingen, Germany

7 Auditory Neuroscience Group, Max Planck Institute for Experimental Medicine, Göttingen, Germany

8 Presynaptogenesis and Intracellular Transport in Hair Cells Group, Institute for Auditory Neuroscience and InnerEarLab, University Medical Center Göttingen, Göttingen, Germany

9 Auditory Systems Physiology Group and InnerEarLab, Department of Otolaryngology, University of Göttingen Medical Center, Göttingen, Germany *Corresponding author. Tel: +49 55139 61128; Fax: +49 551 3912950; E-mail: carolin.wichmann@med.uni-goettingen.de

**Corresponding author. Tel: +49 551 3912379; Fax: +49 551 3912346; E-mail: i.milosevic@eni-g.de

***Corresponding author. Tel: +49 55139 63070; Fax: +49 551 3912950; E-mail: tmoser@gwdg.de

${ }^{\dagger}$ These authors contributed equally to this work 
of endocytic membrane retrieval-rapid (300 ms), fast (4 s), and slow (20 s half-time recovery) - have been described for IHCs (Moser \& Beutner, 2000; Beutner et al, 2001; Neef et al, 2014). However, to date, knowledge of the molecular entities mediating these kinetically distinct forms of endocytosis in IHCs remains scarce. In line with findings at conventional synapses (Ferguson \& De Camilli, 2012; Kononenko \& Haucke, 2015), work on endocytosis in IHCs has indicated a role of dynamins (Boumil et al, 2010; Neef et al, 2014), synaptojanin-1 (Trapani et al, 2009), and clathrin (Siegel \& Brownell, 1986; Neef et al, 2014; Jung et al, 2015) in slow endocytosis that most likely represents clathrin-mediated endocytosis [CME, recently reviewed in Pangrsic and Vogl (2018)].

Surprisingly, genetic disruption of the clathrin adaptor AP-2 did not noticeably affect endocytic membrane retrieval in IHCs (Jung et al, 2015). However, in AP- $2 \mu$ mutants, the abundance of clathrincoated structures near the presynaptic AZs was reduced and large membranous organelles (endosome-like vacuoles, ELVs) accumulated after stimulation (Jung et al, 2015) similar to findings at conventional synapses (Kononenko et al, 2014). This supports the notion that, next to CME, bulk retrieval (reviewed in Kokotos and Cousin (2015)) may play a prominent role in hair cells (Lenzi et al, 2002; Neef et al, 2014; Revelo et al, 2014; Jung et al, 2015). SVs are then rapidly reformed from endocytosed membranes (Kamin et al, 2014; Revelo et al, 2014), which seems to employ clathrin-dependent and clathrin-independent mechanisms (Jung et al, 2015). Importantly, the processes of exocytosis and endocytosis are intimately coupled and tightly coordinated-both at classical neuronal and IHC ribbon synapses - and the proper function of both types of synapses depends on this coupling (Haucke et al, 2011; Wichmann \& Moser, 2015; Milosevic, 2018). In IHCs, AP-2, which interacts with otoferlin (Duncker et al, 2013; Jung et al, 2015), has been implicated in exocytosis and endocytosis coupling (Jung et al, 2015).

In neurons, a range of molecular key players have been identified that orchestrate endocytic membrane retrieval and SV reformation (Kononenko \& Haucke, 2015; Milosevic, 2018); yet, their respective relevance for these processes in IHCs remains unclear. In this context, one interesting molecular target is the evolutionary conserved family of endophilin-A proteins (henceforth "endophilin”), which are involved in endocytic membrane retrieval and uncoating in neurons of invertebrates (Verstreken et al, 2002, 2003; Schuske et al, 2003) and mammals (Milosevic et al, 2011; Watanabe et al, 2018). The current view on mammalian endophilins (A1-A3) pictures them as hubs of a protein network that coordinates cargo packing, bud constriction, actin assembly, and recruitment of factors needed for fission and uncoating (Saheki \& Camilli, 2012). Structurally, endophilins contain a BAR domain that senses and induces membrane curvature, as well as a SH3 domain that recruits the GTPase dynamin and the $\mathrm{PI}(4,5) \mathrm{P}_{2}$ phosphatase synaptojanin-1 to clathrin-coated pits (Verstreken et al, 2002, 2003; Schuske et al, 2003; Perera et al, 2006; Ferguson et al, 2009; Simunovic et al, 2017). Upon fission, $\mathrm{PI}(4,5) \mathrm{P}_{2}$ degradation initiates the shedding of clathrin adaptor proteins from the endocytosed membranes, ultimately leading to the uncoating of SVs (Schuske et al, 2003; Verstreken et al, 2003; Milosevic et al, 2011; Pechstein et al, 2015; Watanabe et al, 2018).

To clarify a potential contribution of endophilins in IHC presynaptic physiology, we performed a comprehensive functional and morphological analysis encompassing single-cell RT-PCR, immunoblotting, electron microscopy, immunohistochemistry, patch-clamp recordings, biochemical interaction studies, and auditory systems physiology using constitutive endophilin knockout mice.

\section{Results}

\section{All three endophilins are expressed in the cochlea}

To investigate the expression of endophilin genes in the organ of Corti and, more specifically, in IHCs, we collected mRNA from IHCs of the apical cochlear coil of Wt mice (C57BL/6J, 2 weeks old, i.e., right after hearing onset). After reverse transcription, we performed single-cell multiplex-nested real-time PCR. In these experiments, all three endophilin-A transcripts (i.e., A1, A2, and A3; Fig 1A and B) could be detected in all tested single IHC samples that were also positive for the housekeeping gene HPRT (see Appendix Table S1 for primer sequences). Importantly, we could not detect endophilin mRNAs in our negative control samples, i.e., a small volume of bath solution that was collected in close proximity to the IHC row prior to and directly after the extraction of the IHC cytoplasm. We note that, while this approach does not provide a quantitative assessment of expression levels due to the nature of the amplification procedure with nested primer pairs, it reliably indicates the presence of endophilin-A1-3 mRNAs in IHCs.

To further investigate endophilin protein expression in situ, we have tested several commercially available as well as custom-made anti-endophilin-A antibodies (see Materials and Methods) in various fixation and permeabilization conditions; yet, we did not obtain specific immunolabeling in the organ of Corti. Therefore, we proceeded to perform immunoblotting with KO-verified antibodies (Milosevic et al, 2011) on cochlear samples of all genotypes using hippocampal and cerebellar tissue extracts as positive controls. Here, we detected bands with the expected molecular weight of endophilin-A1 ( $\sim 39 \mathrm{kD})$ and endophilin-A2 $(\sim 42 \mathrm{kD})$ in $\mathrm{Wt}$ cochleae. These bands were absent in cochlear lysates of the respective KO genotypes, hence strongly suggesting target specificity (Fig 1C- $\mathrm{C}^{\prime} ; \gamma$-adaptin was used as an independent loading control). Taken together, these data indicate the expression of endophilinsA1-3 in IHCs and show the presence of endophilin-A1 and endophilin-A2 protein in the murine cochlea.

\section{Endophilin promotes $\mathrm{Ca}^{2+}$ influx and efficient SV replenishment in IHCs}

Next, we employed perforated patch-clamp recordings to assess the role of endophilins in presynaptic IHC function. Since the cumulative loss of all three endophilin genes is perinatally lethal, we first prepared organotypic cultures of organs of Corti harvested from endophilin-A1/2/3 triple KO (TKO; see Materials and Methods for exact genetic descriptions and breeding schemes of endophilin mutants) and endophilin-A1/3 double KO (1/3DKO) mice, as well as C57BL/6J pups within 3-8 h after birth. Thereafter, organs of Corti were maintained in culture for 1 week to enable synaptic maturation and the otoferlin-dependence of exocytosis to be established (Sobkowicz et al, 1982; Vogl et al, 

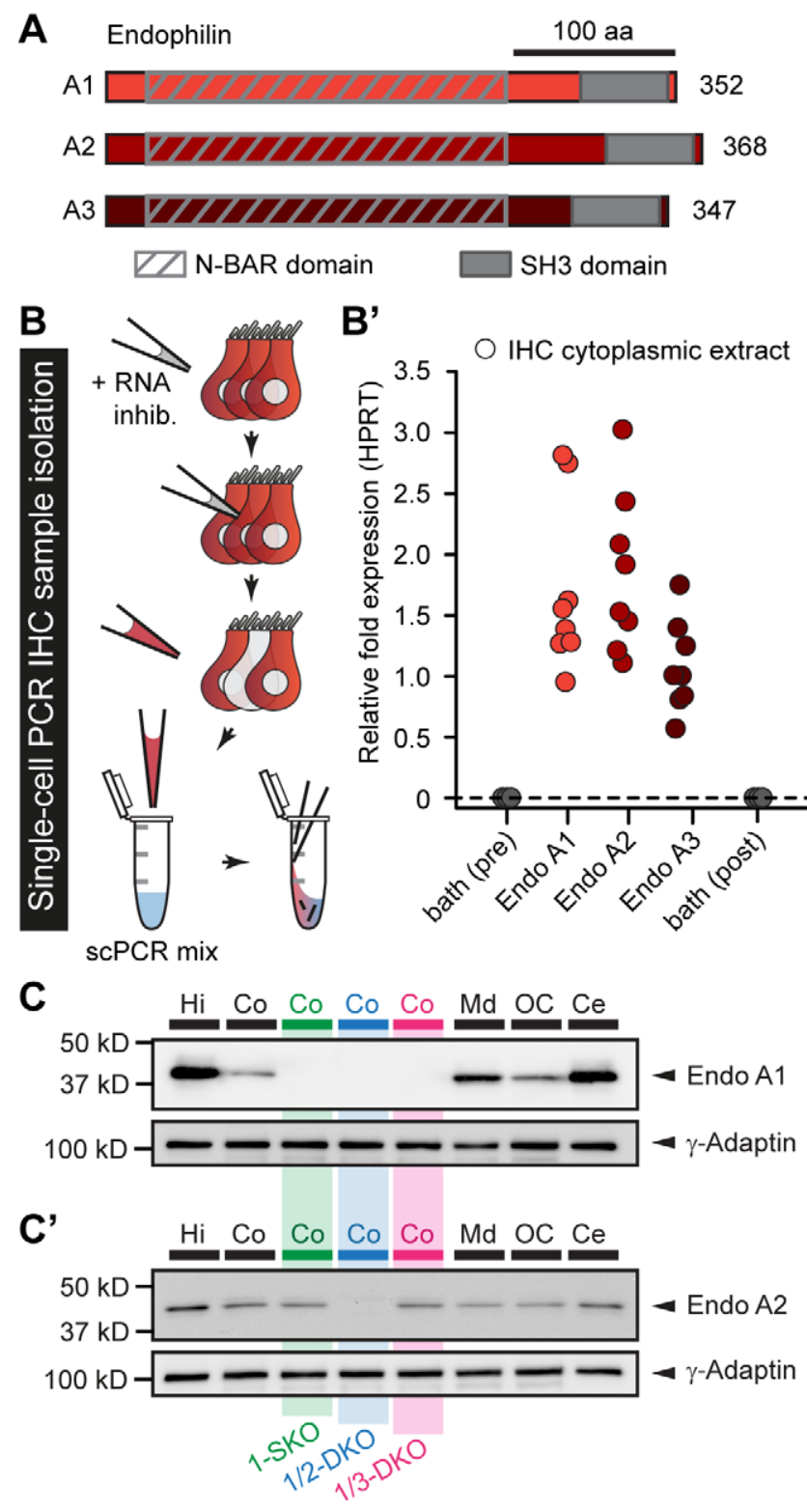

2015) prior to detailed electrophysiological analysis (Fig $2 A^{\prime}$ ). In order to boost depolarization-induced exocytosis, whole-cell $\mathrm{Ca}^{2+}$ currents $\left(\mathrm{I}_{\mathrm{Ca}}\right)$ and the ensuing exocytic membrane capacitance changes $\left(\Delta \mathrm{C}_{\mathrm{m}}\right)$ were recorded at an elevated extracellular $\mathrm{Ca}^{2+}$ concentration of $10 \mathrm{mM}\left(\left[\mathrm{Ca}^{2+}\right]_{\mathrm{e}} ; 1.3 \mathrm{mM}\right.$ is considered physiological). In these experiments, IHCs of $1 / 3$-DKOs exhibited a $25 \%$ reduction of the presynaptic $\mathrm{I}_{\mathrm{Ca}}$ and TKO IHCs showed a nonsignificant trend toward smaller $\mathrm{I}_{\mathrm{Ca}}$ (Fig 2A and $\mathrm{A}^{\prime \prime}, \mathrm{I}_{\max }$ : $-317 \pm 27.3 \mathrm{pA}$ for TKO IHCs, $-298 \pm 22.9 \mathrm{pA}$ for $1 / 3-\mathrm{DKO}$, and $-403 \pm 32.2 \mathrm{pA}$ for $\mathrm{Wt}$; one-way ANOVA, $F(2,26)=3.89$, $P=0.0334$; post hoc Tukey's test: $P=0.046 \mathrm{Wt}$ versus $1 / 3-\mathrm{DKO}$; $P=0.103 \mathrm{Wt}$ versus TKO for the maximal $\mathrm{I}_{\mathrm{Ca}}$ elicited by depolarization to $-17 \mathrm{mV}$ ). Interestingly, $\mathrm{I}_{\mathrm{Ca}}$ of $\mathrm{TKO}$ IHCs showed enhanced inactivation, as evident from a significantly reduced fraction of $\mathrm{I}_{\mathrm{Ca}}$ remaining at $100 \mathrm{~ms}$ of depolarization, which was

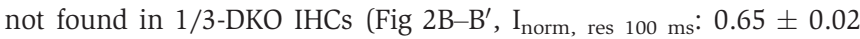
for TKO IHCs, $0.73 \pm 0.02$ for $1 / 3-\mathrm{DKO}$, and $0.71 \pm 0.02$ for Wt;
Figure 1. Endophilin-A expression in the cochlea.

A Schematic domain overview of endophilins-A1-3, highlighting the BAR and $\mathrm{SH} 3$ domains.

B-B' Schematic overview of the sample collection procedure for single-cell RT-PCR (SCPCR). Single IHC cytoplasms from acutely dissected organs of Corti of C57BL/6J (Wt) mice after hearing onset were aspirated and processed for SCPCR as depicted. ( $\left.B^{\prime}\right)$ Expression analysis of endophilinsA1-3 from individually isolated IHC cytoplasms using RT-PCR from a representative experimental run. Please note that for these experiments, negative bath control samples from before and after the isolation procedure were an essential requirement to ensure lack of contamination from cellular debris in the bath solution. HPRT was used as a housekeeping gene.

C-C' Immunoblotting of tissue lysates from postnatal day (p) $15 \mathrm{Wt}(\mathrm{C} 57 \mathrm{BL} / 6 \mathrm{6})$, 1-SKO, 1/2-DKO, and 1/3-DKO revealed protein expression of endophilin$\mathrm{A} 1$ and endophilin-A2 in the murine cochlea and ensured antibody specificity. Unfortunately, none of the commercially available endophilin-A3 antibodies we tested in these experiments gave a specific signal for A3 in cochlear extracts, but rather appeared to (also) detect A1. $\gamma$-Adaptin was used as loading control. All antibody epitopes localize to the distinct $\mathrm{C}$-terminal regions of the different endophilin-A family members. $\mathrm{Hi}$, hippocampus; Ce, cerebellum; Co, pooled cochleae from a single individual of the indicated genotype; Md, modioli (micro-dissected and pooled from $10 \mathrm{Wt}$ animals); OC, organs of Corti (micro-dissected and pooled from $10 \mathrm{Wt}$ animals).

Source data are available online for this figure.

one-way ANOVA, $F(2,26)=4.89, P=0.0158$; post hoc Tukey's test: $P=0.046$ for TKO versus Wt; $P=0.794$ for $1 / 3$-DKO versus $\mathrm{Wt}$ ). Such $\mathrm{I}_{\mathrm{Ca}}$ reduction and enhanced $\mathrm{I}_{\mathrm{Ca}}$ inactivation suggest $\mathrm{a}$ functional interaction of endophilins and $\mathrm{Ca}^{2+}$ channel complexes, which is in line with previous biochemical interaction studies (Chen et al, 2003).

Recordings of exocytic changes in membrane capacitance $\left(\Delta \mathrm{C}_{\mathrm{m}}\right)$ showed impaired exocytosis. Exocytosis of the readily releasable pool (RRP), as approximated by $\Delta \mathrm{C}_{\mathrm{m}}$ responses to 20 ms depolarizations, was significantly attenuated in $1 / 3$ DKO IHCs and tended to be reduced in TKO IHCs (one-way ANOVA, F(3, $33)=5.35, P=0.0041$; post hoc Tukey's test: $P=0.075$ for $\mathrm{Wt}$ versus TKO; $P=0.006$ for $\mathrm{Wt}$ versus $1 / 3-\mathrm{DKO}$ ). Similarly, sustained exocytosis, probed by 100-ms-long depolarizations, tended to be attenuated in both genotypes (Fig 2C-C", KruskalWallis statistic $(\mathrm{KWS})=10.93, P=0.0121$; post hoc Dunn's test: $P=0.220$ for Wt versus TKO; $P=0.025$ for $W t$ versus $1 / 3$-DKO). In contrast, no significant difference was found for responses to short stimuli ( $<10 \mathrm{~ms}$, also see Appendix Tables S2 and S3), indicating that endophilins are dispensable for SV fusion. In order to disentangle the reduction of exocytosis caused by diminished $\mathrm{I}_{\mathrm{Ca}}$ from a potential impairment of SV replenishment in the absence of endophilin-A1 and endophilin-A3, we attempted to match the decreased $\mathrm{I}_{\mathrm{Ca}}$ amplitudes by performing additional recordings from $\mathrm{Wt}(\mathrm{C} 57 \mathrm{BL} / 6 \mathrm{~J}) \mathrm{IHCs}$ at lower $\left[\mathrm{Ca}^{2+}\right]_{\mathrm{e}}$ (i.e., $6 \mathrm{mM}$ instead of $10 \mathrm{mM}$; Fig $2 \mathrm{C}^{\prime}-\mathrm{D}$ ). Under these conditions, $\mathrm{I}_{\mathrm{Ca}}$ of $\mathrm{Wt}$ IHCs closely resembled the ones of endophilin-deficient mutant IHCs. However, the extent of exocytosis from Wt IHCs still exceeded that of cultured 1/3-DKO and TKO IHCs for depolarizations $\geq 10 \mathrm{~ms}$ and remained comparable to the data acquired at $10 \mathrm{mM}\left[\mathrm{Ca}^{2+}\right]_{\mathrm{e}}$ (Fig $\left.2 \mathrm{C}^{\prime}-\mathrm{C}^{\prime \prime}\right)$. Hence, the reduction in $\mathrm{I}_{\mathrm{Ca}}$ in the endophilin mutants cannot fully account for the observed impairment of exocytosis, suggesting an additional requirement for 
A

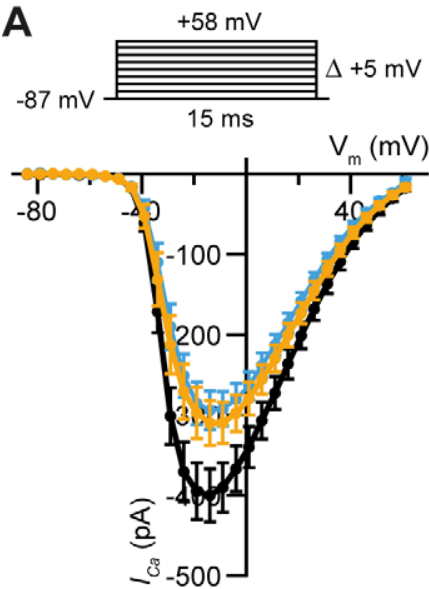

B
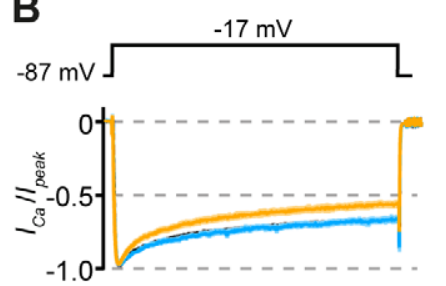

$20 \mathrm{~ms}$
A'
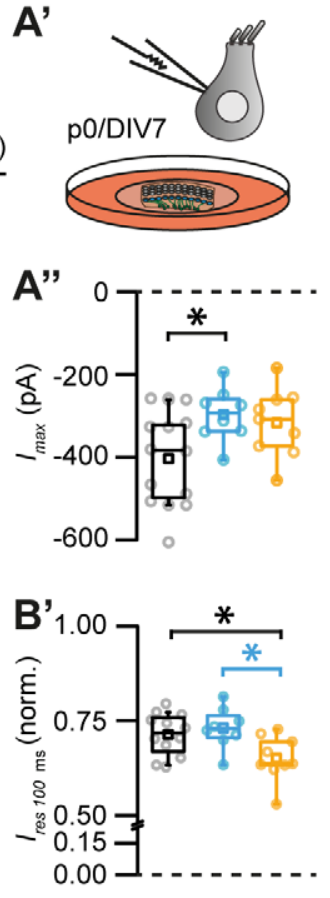
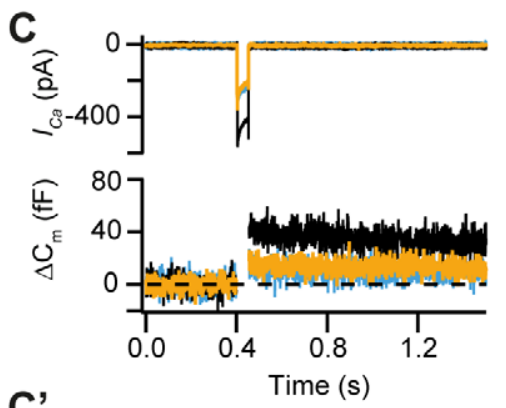

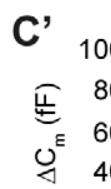
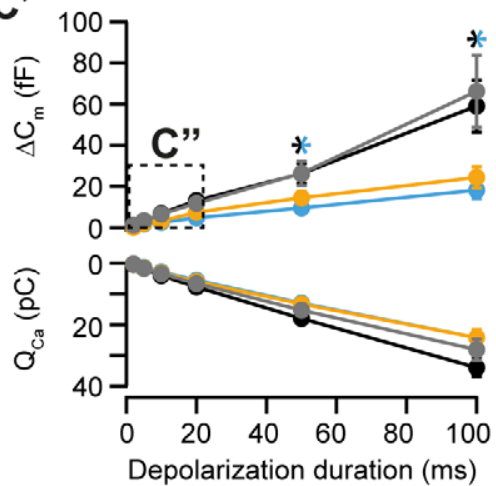

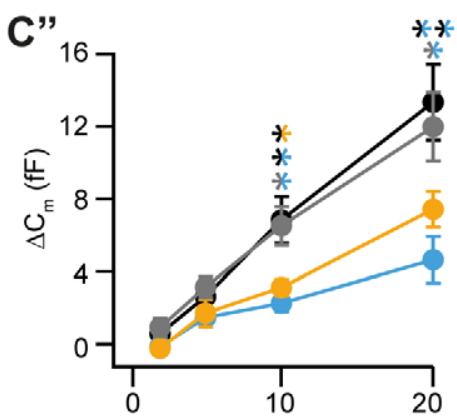

Depolarization duration ( $\mathrm{ms}$ )

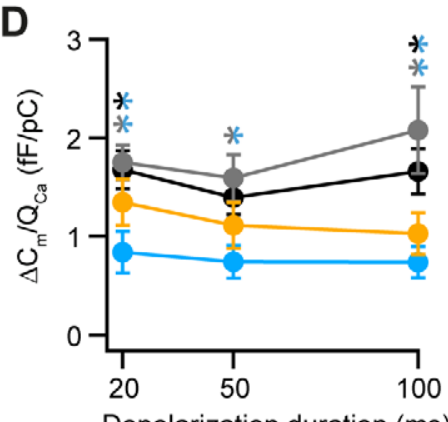

Wt $10 \mathrm{mM}\left[\mathrm{Ca}^{2+}\right]_{e}(\mathrm{n}=12 ; \mathrm{N}=9 ; \mathrm{C}=5)$

Wt $6 \mathrm{mM}\left[\mathrm{Ca}^{2+}\right]_{e}(n=8 ; N=5 ; C=4)$

$\rightarrow$ 1/3-DKO $10 \mathrm{mM}\left[\mathrm{Ca}^{2+}\right]_{e}(\mathrm{n}=8 ; \mathrm{N}=7 ; \mathrm{C}=3)$

1/2/3-TKO $10 \mathrm{mM}\left[\mathrm{Ca}^{2+}\right]_{\mathrm{e}}(\mathrm{n}=9 ; \mathrm{N}=7 ; \mathrm{C}=4)$

Figure 2. Reduced presynaptic $\mathrm{Ca}^{2+}$ currents and exocytosis in endophilin-deficient IHCs maintained in organotypic culture.

A-A" Ca ${ }^{2+}$ current-voltage relationships evoked by incremental $15-\mathrm{ms}$ step depolarizations from $-87 \mathrm{mV}$ to $+58 \mathrm{mV}$ revealed a $\sim 25 \%$ reduction of $\mathrm{I}_{\mathrm{Ca}}$ in $1 / 3 \mathrm{DKO}$ and TKO mice. $\left(A^{\prime}\right)$ Due to the perinatal lethality of TKO mice, detailed electrophysiological characterization of TKO IHCs had to be performed on organotypically cultured organs of Corti after 7 days in vitro (DIV). C57BL/6] (Wt) and 1/3-DKO served as controls. Please note that all recordings from cultured IHCS were performed at $\left[\mathrm{Ca}^{2+}\right]_{\mathrm{e}}$ of $10 \mathrm{mM}$ to maximize IHC exocytic performance. (A") Quantification and statistical analysis of individual maximum $\mathrm{I}_{\mathrm{Ca}}$ amplitudes $\left(I_{\max }\right)$ of the respective genotypes revealed a significant reduction in $I_{\max }$ in both endophilin mutant genotypes $\left({ }^{*} P=0.046\right.$, one-way ANOVA with post hoc Tukey's test).

B-B $\mathrm{B}^{\prime} \quad \mathrm{Ca}^{2+}$ current inactivation was probed by test pulses of $100 \mathrm{~ms}$ to the $I_{\max }$ potential and revealed a significantly stronger inactivation phenotype in TKO IHCs when directly compared to Wt and 1/3-DKO cells. (B') Quantification and statistical analysis of the residual current $\left(I_{\text {res }} 100 \mathrm{~ms}\right)$ at the end of the test pulse ${ }^{\star} P=0.046$, one-way ANOVA with post hoc Tukey's test).

$C-C^{\prime \prime}$ Representative $\mathrm{I}_{\mathrm{Ca}}$ (upper panel) and $\mathrm{C}_{\mathrm{m}}$ (lower panel) in response to a $50 \mathrm{~ms}$ depolarizing step to the potential eliciting $I_{\max }$. $\left(\mathrm{C}^{\prime}\right)$ Exocytic $\Delta \mathrm{C}_{\mathrm{m}}$ and corresponding $\mathrm{Q}_{\mathrm{ca}}$ elicited by depolarizations of stimulus durations from 2 to $100 \mathrm{~ms}$ for all respective genotypes and at $\left[\mathrm{Ca}^{2+}\right]_{\mathrm{e}}=6 \mathrm{mM}$ for a second set of recordings from wild-type IHCS to experimentally approximate the decreased $I_{C a}$ observed in the endophilin mutants. $\left(C^{\prime \prime}\right)$ Magnification of the initial, short depolarizing steps (2$20 \mathrm{~ms}$ ) for clarity. Exocytic $\Delta C_{m}$ of cultured endophilin-deficient IHCs was strongly reduced ( ${ }^{*} P<0.05$; ${ }^{\star *} P<0.01$; one-way ANOVA with post hoc Tukey's or nonparametric K-W with post hoc Dunn's test; please also refer to Appendix Tables S2 and S3 for detailed statistical analysis).

D The reduced $\mathrm{Ca}^{2+}$ efficiency of exocytosis $\left(\Delta \mathrm{C}_{\mathrm{m}} / \mathrm{Q}_{\mathrm{Ca}}\right)$ in endophilin-deficient IHCs indicates that diminished $\mathrm{Ca}^{2+}$ influx cannot fully account for the reduction of exocytosis $\left({ }^{*} P<0.05\right.$; ${ }^{* *} P<0.01$; one-way ANOVA with post hoc Tukey's or non-parametric K-W with post hoc Dunn's test; please also refer to Appendix Table $S 4$ for detailed statistical analysis).

Data information: For panels (A-D), the following numbers of replicates were used: Wt $10 \mathrm{mM}\left[\mathrm{Ca}^{2+}\right]_{\mathrm{e}}$ number of cells $(n)=12$, number of animals $(\mathrm{N})=9$, number of organotypic cultures (C) $=5$; Wt $6 \mathrm{mM}\left[\mathrm{Ca}^{2+}\right]_{\mathrm{e}} n=8 / \mathrm{N}=5 / \mathrm{C}=4$; 1/3-DKO $10 \mathrm{mM}\left[\mathrm{Ca}^{2+}\right]_{\mathrm{e}} n=8 / \mathrm{N}=7 / \mathrm{C}=3$; TKO $10 \mathrm{mM}\left[\mathrm{Ca}{ }^{2+}\right]_{\mathrm{e}} n=9 / \mathrm{N}=7 / \mathrm{C}=4$. Error bars in $\left(\mathrm{C}^{\prime}-\mathrm{D}\right)$ indicate the SEM; box plots in $\left(\mathrm{A}^{\prime \prime}\right)$ and $\left(\mathrm{B}^{\prime}\right)$ illustrate the median with the interquartile range, whiskers indicate $10-90 \%$ of data points, and the squares present the respective mean value.

endophilin-A1 and endophilin-A3 in exocytosis, e.g., in vesicle replenishment. This is further illustrated by plotting $\Delta \mathrm{C}_{\mathrm{m}}$ versus the corresponding charge of $\mathrm{I}_{\mathrm{Ca}}$, which shows a reduced efficiency of $\mathrm{I}_{\mathrm{Ca}}$ to drive exocytosis (Fig 2D; individual statistics for all depolarization durations can be found in Appendix Table S4). Taken together, patch-clamp analysis of organotypically cultured IHCs from endophilin mutants revealed reduced $\mathrm{I}_{\mathrm{Ca}}$ amplitudes alongside altered $\mathrm{I}_{\mathrm{Ca}}$ inactivation and impaired exocytosis. Moreover, our data imply a partial functional dispensability of endophilin-A2 in the presence of endophilin-A1 and endophilin-A3, since both $\mathrm{I}_{\mathrm{Ca}}$ and $\Delta \mathrm{C}_{\mathrm{m}}$ amplitudes were statistically indistinguishable between 1/3-DKO and TKO IHCs.

Guided by these results, we focused our further perforated patchclamp studies on $\mathrm{I}_{\mathrm{Ca}}$ and exocytosis of 1-SKO and 1/3-DKO IHCs after hearing onset (at p14-17) and at near physiological $\left[\mathrm{Ca}^{2+}\right]_{\mathrm{e}}$ ( $2 \mathrm{mM})$. Analogous to our observations from cultured immature IHCs, we found a statistically significant reduction of $\mathrm{I}_{\mathrm{Ca}}$ of $\sim 20 \%$ for 1-SKO and 1/3-DKO IHCs under these conditions (Fig 3A-A'; $\mathrm{I}_{\max }:-128 \pm 5.0 \mathrm{pA}$ for 1 -SKO IHCs, $-129 \pm 2.1 \mathrm{pA}$ for $1 / 3-\mathrm{DKO}$, and $-153 \pm 5.0 \mathrm{pA}$ for Wt; KWS $=18.72 ; P<0.0001$; post hoc 

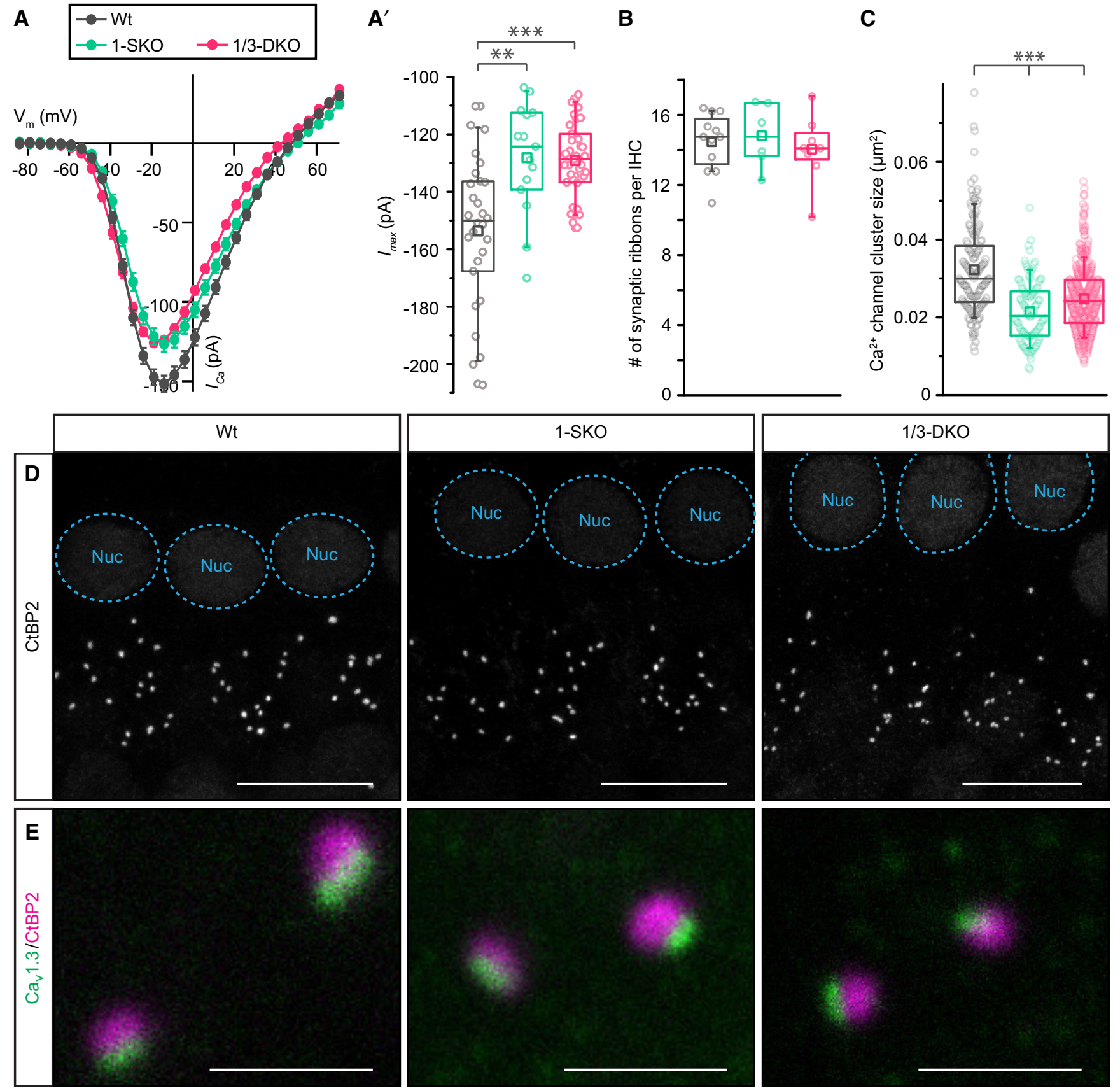

Figure 3. Disruption of endophilins reduces $\mathrm{Ca}^{2+}$ influx of $\mathrm{IHCs}$ after hearing onset.

A Ca ${ }^{2+}$ current-voltage relationships in response to 10-ms step depolarizations. ( $\left.A^{\prime}\right)$ The peak of $\mathrm{Ca}^{2+}$ influx was significantly reduced in endophilin 1 -SKO

$\left.{ }^{* *} P=0.0024\right)$ and $1 / 3-$ DKO $\left.{ }^{* \star \star} P=0.0003\right)$ when compared to Wt $(\mathrm{Wt} n=30 / \mathrm{N}=20 ; 1-\mathrm{SKO} n=15 / \mathrm{N}=9 ; 1 / 3-\mathrm{DKO} n=39 / \mathrm{N}=20$; non-parametric K-W with post hoc Dunn's correction).

B Quantification of CtBP2-labeled ribbons revealed a number of approximately 14-15 ribbon synapses per IHC comparable across all genotypes (Wt $n=12 / \mathrm{N}=9$; 1SKO $n=6 / N=4 ; 1 / 3-$ DKO $n=8 / N=6$; one-way ANOVA, $P=0.717$ ).

C Quantification of the cross-sectional area of Cav1.3 immunofluorescence revealed approx. 34\% smaller clusters in 1-SKOs and 24\% smaller clusters in 1/3-DKOs (Wt $n=153 / \mathrm{N}=2 ; 1-\mathrm{SKO} n=102 / \mathrm{N}=2 ; 1 / 3-\mathrm{DKO} n=441 / \mathrm{N}=4 ;{ }^{* \star *} \mathrm{P}<0.0001 ; \mathrm{K}-\mathrm{W}$ and post hoc Dunn's test).

D Representative maximum projections of confocal sections from organs of Corti of p15 Wt, 1-SKO, and 1/3-DKO mice stained for CtBP2 to label synaptic ribbons. Please note that CtBP2 expression is also found in the nucleus (nuclei are highlighted by dashed circles in the individual panels). Scale bars: $10 \mu \mathrm{m}$.

E 2D STED images of IHC ribbon synapses stained for CtBP2 (magenta) and Cav1.3 (green). Cav1.3 Ca ${ }^{2+}$ channels remain clustered at AZs of 1-SKO and 1/3-DKO IHCs. Scale bars: $1 \mu \mathrm{m}$.

Data information: Box plots in $\left(A^{\prime}-C\right)$ illustrate the median with the interquartile range, whiskers indicate $10-90 \%$ of data points, and the squares present the respective mean value. 
Dunn's test: $P=0.0024$ for Wt versus 1 -SKO and $P=0.0003$ for $\mathrm{Wt}$ versus $1 / 3$-DKO for the maximal $\mathrm{I}_{\mathrm{Ca}}$ elicited by depolarization to $-14 \mathrm{mV}) . \mathrm{I}_{\mathrm{Ca}}$ inactivation at $200 \mathrm{~ms}$ of depolarization was not altered in 1-SKO, 1/2 DKO, and 1/3-DKO IHCs (Fig EV1; $\mathrm{I}_{\text {norm, res } 200 \mathrm{~ms}}: 0.72 \pm 0.02$ for 1 -SKO IHCs, $0.74 \pm 0.01$ for $1 / 2$-DKO, $0.73 \pm 0.02$ for $1 / 3$-DKO, and $0.70 \pm 0.02$ for Wt; one-way ANOVA, $F(3,55)=0.95, P=0.4218)$, likely suggesting functional redundancy between the different endophilin genes.

In order to explore potential reasons for the observed reduction of $\mathrm{I}_{\mathrm{Ca}}$, such as a decreased number of IHC synapses or a lower abundance of $\mathrm{Ca}_{\mathrm{V}} 1.3 \mathrm{Ca}^{2+}$ channels per $\mathrm{AZ}$, we performed semi-quantitative immunohistochemistry on IHCs from 2-week-old Wt, 1-SKO, and $1 / 3$-DKO mice. Firstly, we counted the number of synaptic ribbons per IHC as a proxy of the number of afferent synapses (Khimich et al, 2005; Meyer et al, 2009, Fig 3B and D) and found no difference between the genotypes (one-way ANOVA, $F(2,23)=0.337$, $P=0.717)$. Secondly, all ribbons appeared anchored at the $\mathrm{AZ}$ (Fig 3E and electron microscopy data in Fig 8A, see below), where presynaptic $\mathrm{Ca}^{2+}$ channels remained clustered (Fig 3E). In line with the reduced $\mathrm{I}_{\mathrm{Ca}}$ amplitudes, super-resolution 2D STED imaging of $\mathrm{Ca}_{\mathrm{V}} 1.3$ immunofluorescence revealed a significant decrease in crosssectional area of presynaptic $\mathrm{Ca}^{2+}$ channel clusters of 1-SKO and 1/3DKO IHCs (Fig 3C and E; Wt: $0.032 \pm 0.001 \mu \mathrm{m}^{2}$ versus 1-SKO: $0.021 \pm 0.001 \mu \mathrm{m}^{2}$ versus $1 / 3$-DKO: $0.024 \pm 0.001 \mu \mathrm{m}^{2} ; \mathrm{KWS}=$ 76.14, $P<0.0001$; post hoc Dunn's test: $P<0.0001$ for Wt versus 1 -SKO and $P<0.0001$ for Wt versus $1 / 3$-DKO).

Next, we tested the exocytic capacity of endophilin-deficient IHC ribbon synapses after the onset of hearing by measuring exocytic $\Delta \mathrm{C}_{\mathrm{m}}$ in response to step depolarizations to $-14 \mathrm{mV}$ eliciting maximal $\mathrm{I}_{\mathrm{Ca}}$ at near physiological $\left[\mathrm{Ca}^{2+}\right]_{\mathrm{e}}(2 \mathrm{mM}$, Fig 4$)$. In line with our observations from organotypically cultured IHCs, we found sustained exocytosis to be reduced in 1/3-DKO IHCs (Fig 4A and B; e.g., $\Delta \mathrm{C}_{\mathrm{m}, 200 \mathrm{~ms}}$ for $1 / 3$-DKO IHCs was $55.11 \pm 4.8 \mathrm{fF}$ versus $88.16 \pm 13.11 \mathrm{fF}$ for Wt IHCs; KWS $=6.03, P=0.0490$; post hoc Dunn's test: $P=0.0470$ for $1 / 3$-DKO versus Wt; individual Tukey's/ post hoc Dunn's results for all other depolarization durations can be found in Appendix Tables S5 and S6). Likewise, when approximating the rate of sustained exocytosis as $\left(\Delta \mathrm{C}_{\mathrm{m}}, 200 \mathrm{~ms}-\Delta \mathrm{C}_{\mathrm{m}, 20 \mathrm{~ms}}\right) /$ $180 \mathrm{~ms}$, we found a tendency for lower rates in endophilin-deficient IHCs $(0.25 \pm 0.02 \mathrm{fF} / \mathrm{ms}$ for $1 / 3-\mathrm{DKO}, 0.27 \pm 0.03 \mathrm{fF} / \mathrm{ms}$ for $1-\mathrm{SKO}$ versus $0.42 \pm 0.06 \mathrm{fF} / \mathrm{ms}$ for $\mathrm{Wt}$; KWS $=5.223, P=0.0734)$. This trend remained also after normalizing to the integrated $\mathrm{I}_{\mathrm{Ca}}\left(\mathrm{Q}_{\mathrm{Ca}}\right)$, suggesting an exocytic deficit beyond that explained by reduced $\mathrm{Ca}^{2+}$ influx, as already seen in cultured IHCs (Fig 4C; e.g., for $200 \mathrm{~ms}: 2.54 \pm 0.19 \mathrm{fF} / \mathrm{pC}$ for $1 / 3$-DKO versus $3.34 \pm 0.52 \mathrm{fF} / \mathrm{pC}$ for 1-SKO versus $3.81 \pm 0.45 \mathrm{fF} / \mathrm{pC}$ for $\mathrm{Wt}$; $\mathrm{KWS}=6.70, P=0.0352$, post hoc Dunn's test: $P=0.0323$ for $1 / 3-\mathrm{DKO}$ versus $\mathrm{Wt}$ and $P>0.99$ for 1-SKO versus Wt; individual post hoc Tukey's/Dunn's results for all other depolarization durations can be found in Appendix Table S7).

Finally, to assess the consequences of endophilin disruption at the level of the auditory system, we recorded auditory brainstem responses (ABRs) from 1-SKO, 1/3-DKO and Wt mice at 6 weeks of age. ABRs reflect the synchronous activation of auditory neurons of the various stages of the early auditory pathway; e.g., Jewett wave 1 represents the compound action potential of the SGNs, and Jewett waves 2 and 3 reflect signal propagation in the cochlear nucleus (Melcher et al, 1996). Curiously, despite the morphological and physiological deficiencies found at the synapses of endophilin-deficient IHCs, ABR thresholds and amplitudes were comparable between Wt, 1-SKO and 1/3 DKO mice (Fig EV2; note that 1/2-DKO and TKO mice could not be tested due to their premature lethality).

In summary, our combined functional and morphological data indicate a role of endophilins in promoting the abundance of $\mathrm{Ca}_{\mathrm{V}} 1.3$ $\mathrm{Ca}^{2+}$ channels at IHC AZs. In addition, our data suggest that endophilins are required for efficient SV replenishment to IHC AZs as required for sustained exocytosis.

\section{Endophilin-A1 interacts with otoferlin, a key player in hair cell exocytosis}

Our patch-clamp recordings implied that the reduced $\mathrm{Ca}^{2+}$ currents seen in endophilin mutants cannot fully account for the deficits in SV replenishment, and hence, we focused our search on putative presynaptic effector molecules that may be regulated by endophilins. One such potential candidate is the multi- $\mathrm{C}_{2}$ domain protein otoferlin-a key player that is essentially required for IHC exocytosis (Roux et al, 2006; Pangrsic et al, 2010; Vogl et al, 2016). Otoferlin has previously been shown to interact with endocytic adaptor protein AP-2 (Duncker et al, 2013; Jung et al, 2015), and this interaction might serve the clearance of release sites after SV fusion (Jung et al, 2015), therefore placing otoferlin in the same subcellular framework where endophilins are thought to operate. We characterized otoferlin levels and its subcellular distribution in endophilin mutant IHCs using semi-quantitative immunohistochemistry (Fig 5). Interestingly, our analysis revealed a reduction of overall IHC otoferlin immunofluorescence intensity by $28.4 \pm 3.5 \%$ in $1 / 3$-DKOs (Fig 5A-C; unpaired $t$-test, $t=6.71, P<0.0001 \mathrm{Wt}$ versus $1 / 3$-DKO) that however was much less pronounced than in IHCs of AP- $2 \mu$ mutants (by approximately 70\%) (Jung et al, 2015). Furthermore, when assessing the subcellular distribution pattern of otoferlin in mutant IHCs via line profile analysis along the longitudinal IHC axis, we found comparable patterns of otoferlin distribution, but a reduction in the overall signal intensity in $1 / 3$ DKO IHCs (Fig 5C). In contrast, the fluorescence intensity of myosin 6, previously characterized as regulator of IHC maturation and interaction partner of otoferlin (Roux et al, 2009) as well as of AP-2, seemed unchanged in response to the disruption of endophilins (Fig EV3 and Appendix Fig S1, respectively).

In light of the reduction of otoferlin even in the partial absence of endophilin, we aimed to better understand the relation of these proteins. Thus, we tested whether otoferlin interacts with endophilin-A1 by immunoprecipitation. An interaction between otoferlin and endophilin-A1 in vitro was revealed by specific enrichment of endophilin in anti-GFP immunoprecipitates from HeLa cells expressing endophilin-A1-mRFP and EGFP-otoferlin (Fig 5D and $\mathrm{D}^{\prime}$ ). Notably, using an alternative experimental approach, bead-coupled EGFP-otoferlin was able to bind highly purified endophilin-A1 (Fig $5 \mathrm{E}$ and $\mathrm{E}^{\prime}$ ), thereby suggesting an interaction of endophilin-A1 and otoferlin in both systems. In IHCs, such an interaction might aid the coupling of exocytosis and endocytosis.

\section{Endophilin is involved in endocytic membrane retrieval in IHCs}

Next, we performed $C_{m}$ measurements to study whether endophilin deficiency alters endocytic membrane retrieval following 
A
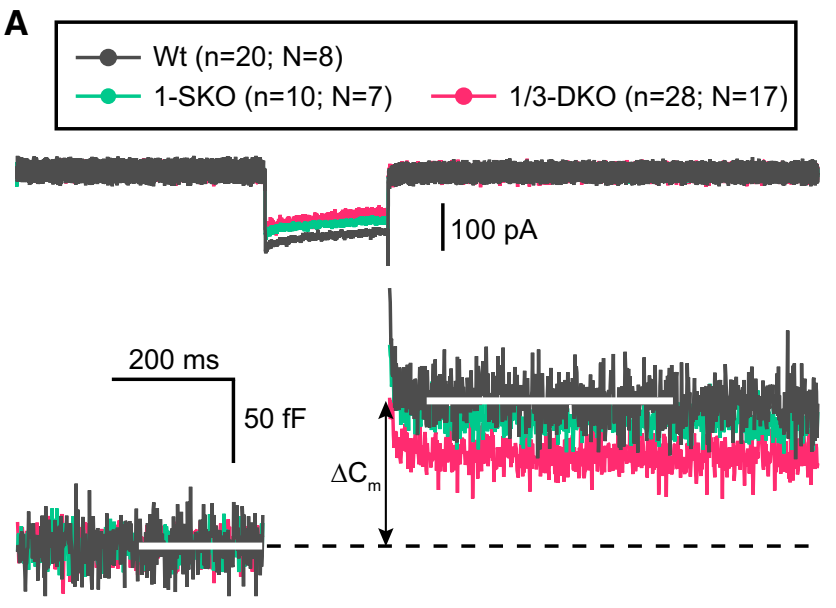

\section{B}

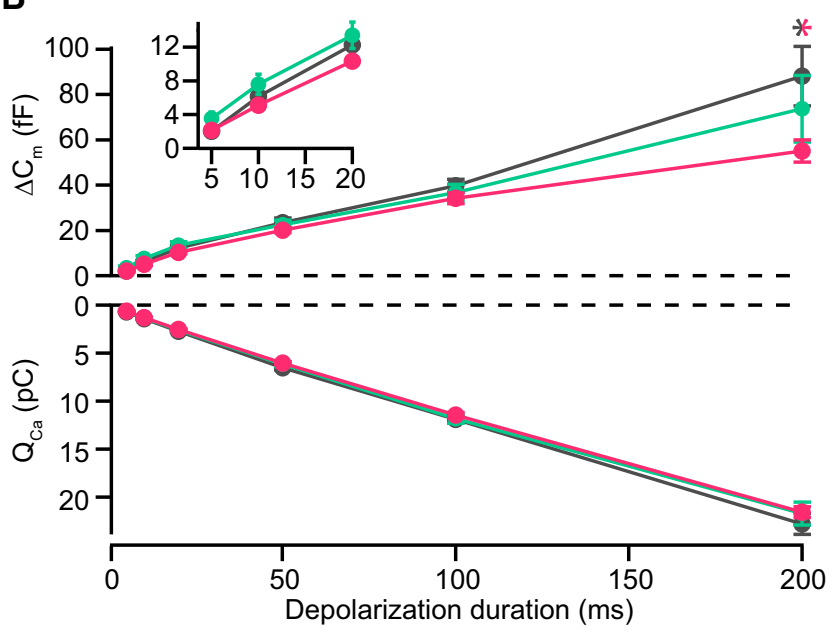

C

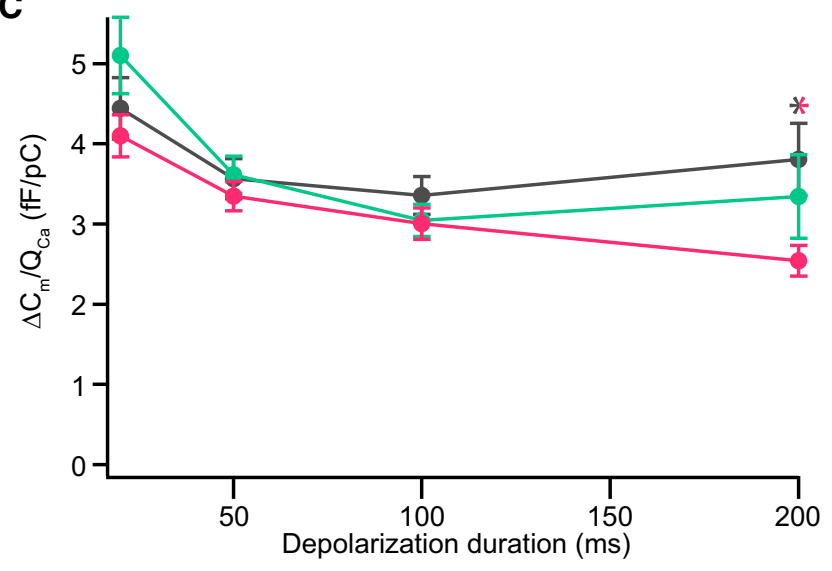

depolarization-induced exocytosis in IHCs after hearing onset. We employed short and long step depolarizations to $-14 \mathrm{mV}$ to trigger different amounts of exocytosis (Fig 6). In IHCs, short depolarizations (20 ms, recruiting the RRP) predominantly result in a slow, near linear post-stimulus $\mathrm{C}_{\mathrm{m}}$ decline back to baseline, which we assume to reflect CME (Neef et al, 2014). Long depolarizations (200 ms) - turning over in excess of 3-4 times the equivalent of the $\mathrm{RRP}$-additionally evoke an exponentially decaying $\mathrm{C}_{\mathrm{m}}$ component that likely involves bulk retrieval (Neef et al, 2014). In these
Figure 4. Sustained exocytosis is impaired in endophilin-deficient IHCs.

A Representative $\mathrm{Ca}^{2+}$ currents (upper panel) and exocytic membrane capacitance increments $\left(\Delta C_{m}\right.$; lower panel) in response to a $200-\mathrm{ms}$ depolarizing step to $-14 \mathrm{mV}$.

B Exocytic $\Delta \mathrm{C}_{\mathrm{m}}$ (top) and corresponding $\mathrm{Ca}^{2+}$ current integrals $\mathrm{Q}_{\mathrm{Ca}}$ (bottom) of $\mathrm{Wt}$ and endophilin-deficient IHCs in response to voltage steps from -84 to $-14 \mathrm{mV}$ of variable stimulus duration (5-200 ms). In 1/3-DKO IHCs, our data indicate reduced sustained exocytosis to a strong depolarization (200 ms; ${ }^{*} P=0.0470, \mathrm{~K}-\mathrm{W}$ with post hoc Dunn's test; please also refer to Appendix Tables S5 and S6 for detailed statistical analysis). Inset: Initial $\Delta C_{m}$ showed comparable RRP exocytosis in endophilin-deficient IHCs.

C Ca ${ }^{2+}$ efficiency to drive exocytosis was reduced for strong depolarizations (200 ms) in 1/3-DKO IHCs ( ${ }^{*} P=0.0323, \mathrm{~K}-\mathrm{W}$ with post hoc Dunn's test; please also refer to Appendix Table $\mathrm{S7}$ for detailed statistical analysis).

Data information: For panels (A-C), the numbers of replicates were Wt $n=20$ / $N=8 ; 1-S K O n=10 / N=7 ; 1 / 3-D K O n=28 / N=17$. Data represent averages \pm SEM.

experiments, we also included 1/2-DKO IHCs into the analysis of the endocytic membrane retrieval as a prominent role of endophilin-A2 in scission and SV reformation had been reported previously (Renard et al, 2015). After a 20-ms depolarization, the endocytic $\mathrm{C}_{\mathrm{m}}$ decline was significantly slowed in IHCs of both $1 / 2$ DKO and 1/3-DKO (Fig 6A, C and D). Here, the mean slope of a line fit to the endocytic $C_{m}$ decline amounted to $-0.51 \mathrm{fF} / \mathrm{s}$ and $-0.50 \mathrm{fF} / \mathrm{s}$ for $1 / 2$-DKO and 1/3-DKO IHCs, respectively, compared to $-0.84 \mathrm{fF} / \mathrm{s}$ in Wt IHCs (Fig 6D; one-way ANOVA, $F(2$, $35)=5.87, P=0.0063$; post hoc Tukey's test: $P=0.0144$ for $1 / 2$ DKO versus $\mathrm{Wt}$ and $P=0.0144$ for $1 / 3-\mathrm{DKO}$ versus $\mathrm{Wt}$ ). Moreover, there was a trend toward a delayed $C_{m}$ return to baseline for IHCs of both mutants that did not reach statistical significance (Fig 6C, one-way ANOVA, $F(2,35)=1.67, P=0.2025$ ). A trend toward slower linear $C_{m}$ decline was also observed in the responses to a 200-ms depolarization (following the exponential phase of retrieval; Fig 6B and D; individual statistics for all endocytosis parameters can be found in Appendix Table S8). These data suggest a reduced rate of membrane retrieval by CME in the absence of endophilins-A1/2 or endophilins-A1/3 in IHCs. In addition, we found a trend toward a smaller and slower exponential phase of $\mathrm{C}_{\mathrm{m}}$ decline in 1/2-DKO and 1/3-DKO IHCs following $200 \mathrm{~ms}$ of depolarization, which however did not reach statistical significance (Fig 6E and F; yet, these data reach statistical significance, if statistical outliers are excluded for the time constant, KWS $=8.52$ and $P=0.0141$ ). In conclusion, our $C_{m}$ measurements indicate a role of endophilins particularly in the slow component of IHC endocytic membrane retrieval, likely reflecting CME.

\section{Endophilin deficiency impairs vesicle uncoating and synaptic vesicle reformation}

In order to further characterize the deficits in endocytosis revealed by electrophysiology, we performed extensive morphological analyses using electron microscopy and electron tomography. Consistent with the immunohistochemical data, the general morphology and plasma membrane anchoring of synaptic ribbons at presynaptic AZs of all genotypes appeared normal (Fig 8A).

In a first step, we used random ultrathin sections to explore the abundance of small, clear, and uncoated vesicles (diameter $<70 \mathrm{~nm}$, likely representing SVs), endosome-like vacuoles (ELVs), 

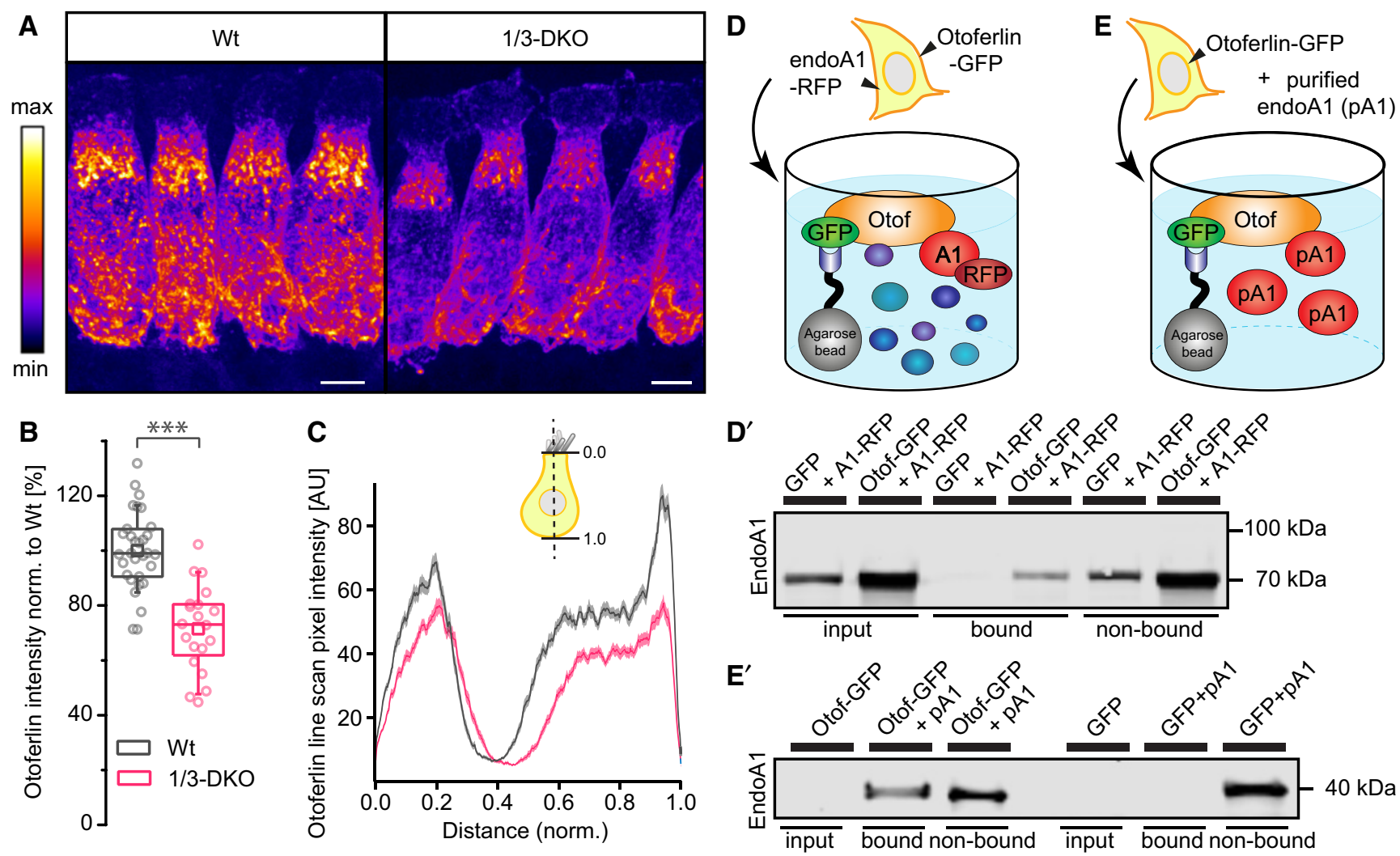

Figure 5. Disruption of endophilins causes a reduction of IHC otoferlin levels.

A Confocal maximum projections of otoferlin-immunolabeled IHCs from p15 Wt and 1/3-DKO mice illustrated with an intensity-coded lookup table where brighter colors indicate higher intensity. Scale bars: $5 \mu \mathrm{m}$.

B Otoferlin levels were reduced by approx. $28.4 \pm 3.5 \%$ in 1/3-DKO IHCS compared to Wt IHCs (relative intensities normalized to Wt $t_{\text {avg }}$; Wt $n=31$ images (229 IHCs)/ $N=5$ organs of Corti (three animals); $1 / 3-D K O n=20$ images (196 IHCS) $/ N=5$ organs of Corti (four animals); unpaired Student's $t$-test; ${ }^{* \star \star} P<0.001$; box plots illustrate the median with the interquartile range, whiskers indicate $10-90 \%$ of data points, and the squares present the respective mean value).

C Normalized otoferlin intensity line profiles through single IHCS of the respective genotypes at a longitudinal central plane through the nucleus from apical (0.0) to basal (1.0) revealed similar distribution patterns of otoferlin in IHCS of Wt and 1/3-DKO, but an overall reduction of otoferlin levels throughout the entire cell in 1/3DKOs. Traces indicate the mean pixel intensity \pm SEM; Wt $n=123 / \mathrm{N}=3 ; 1 / 3-\mathrm{DKO} n=124 / \mathrm{N}=3$.

$D$, E Otoferlin interacts with endophilin-A1. (D, D') Interaction of otoferlin and endophilin-A1 detected by co-IP in HeLa cells co-expressing GFP-otoferlin and RFPendophilin-A1. Otoferlin-GFP was immunoprecipitated (IP) by GFP-Trap beads, and blots were probed with a KO-validated anti-endophilin-A1 antibody. (E, E') Exogenously overexpressed GFP-otoferlin was immunoprecipitated using GFP-Trap beads and incubated with purified endophilin-A1 (pA1). IP was then followed by immunoblotting with an anti-endophilin-A1 antibody.

Source data are available online for this figure.

and clathrin-coated membrane profiles within a radius of $1 \mu \mathrm{m}$ from the synaptic ribbon (Fig 7A). For this analysis, we excluded the SVs directly adjacent to the presynaptic ribbon and considered them in a separate analysis (see below). The total number of small uncoated cytosolic vesicles was elevated in 1-SKO IHCs, but reduced in 1/2-DKO IHCs, while 1/3-DKOs displayed unaltered vesicle counts compared to Wt (Fig 7B; KWS $=130, P<0.0001$; post hoc Dunn's results can be found in Appendix Table S9). Additionally, the area covered by ELVs was increased in 1/2-DKO and 1/3-DKO (Fig 7C; KWS $=70.17, P<0.0001$; post hoc Dunn's results can be found in Appendix Table S9), suggesting compromised reformation of SVs from ELVs in these genotypes. Quantification of clathrin-coated profiles included the following: (i) coated vesicles with diameters $<70 \mathrm{~nm}$, (ii) fully coated vacuoles with diameters $>70 \mathrm{~nm}$, (iii) coated pits at the plasma membrane, and (iv) coated pits budding from ELVs. We observed an overall significantly increased number of coated structures in IHCs of 1SKOs, and, even more prevalently, in 1/3-DKOs and 1/2-DKO compared to Wt (Fig 7D; KWS $=66.1, P<0.0001$ ). While 1 -SKO AZs exhibited more coated vesicles, numbers of coated vacuoles and coated pits budding from the plasma membrane were increased at AZs of both DKOs (Fig 7D'; coated SVs: KWS = 27.13, $P<0.0001$; coated vacuoles: KWS $=33, P<0.0001$; coated pits: KWS $=79.67, P<0.0001$; coated pits at ELVs: $K W S=6.503$, $P=0.0895$; post hoc Dunn's results for all data sets can be found in Appendix Table S9). These data point towards impaired SV uncoating, taking effect already in 1-SKOs as well as additional deficits in fission and/or membrane bending in $1 / 2$-DKOs and $1 / 3$ DKOs during CME.

Secondly, we analyzed small vesicles in direct ribbon proximity (diameters $<70 \mathrm{~nm}$, clear, and uncoated), hereafter categorized as SVs, using electron microscopy of random ultrathin sections 
A
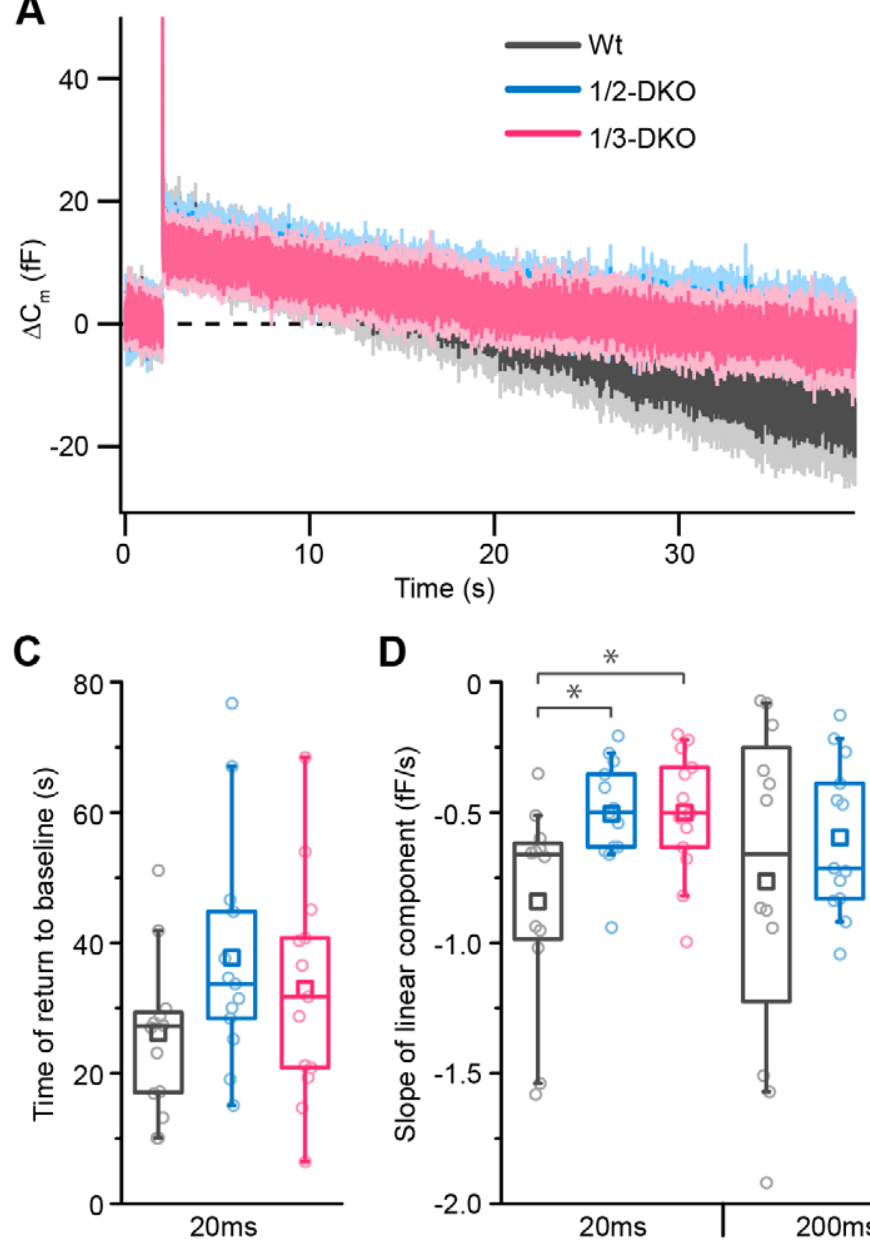

D

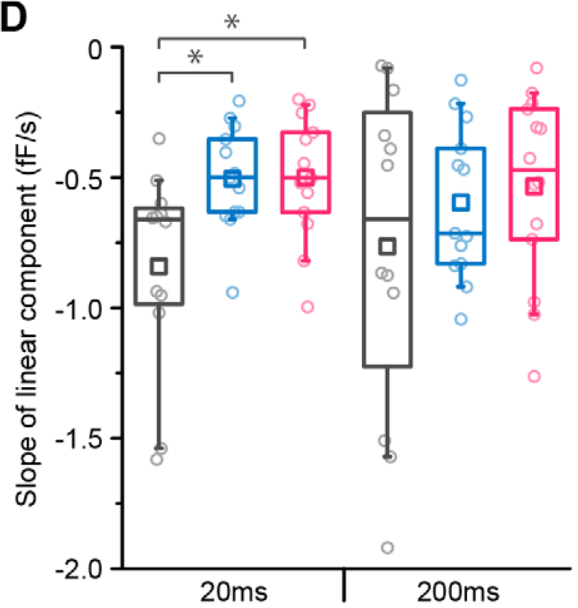

B

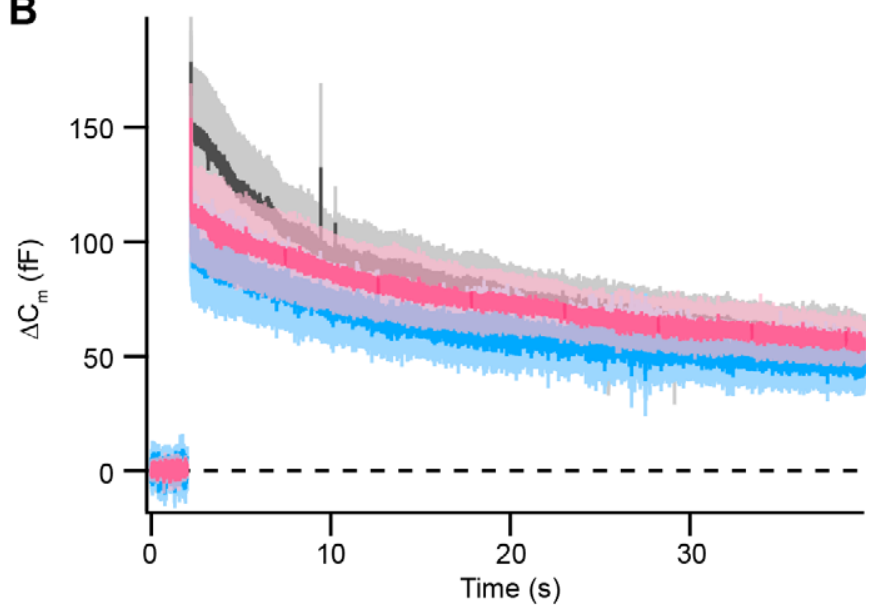

E

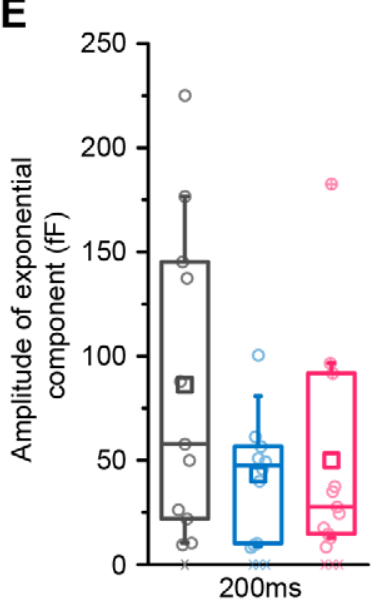

$\mathbf{F}$

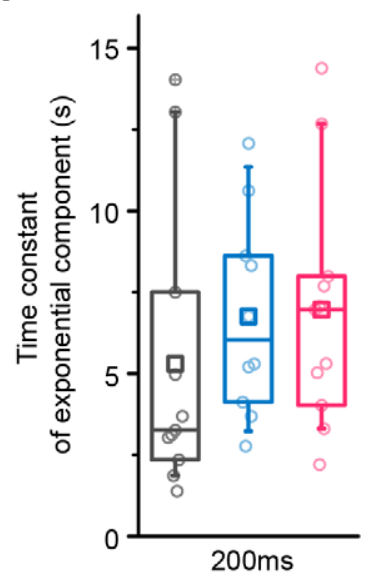

Figure 6. Slowed endocytic membrane retrieval in endophilin-deficient IHCs.

A, B Endocytosis was assessed by determining the decrease in $C_{m}$ during 20 s post-depolarization in perforated patch-clamp recordings from $\mathrm{Wt}$, $1 / 2-\mathrm{DKO}$ and $1 / 3-\mathrm{DKO}$ IHCS. The two kinetic components are presented as average $\Delta \mathrm{C}_{\mathrm{m}}$ recordings after (A) $20 \mathrm{~ms}(\mathrm{Wt} n=12 / \mathrm{N}=8 ; 1 / 2-\mathrm{DKO} n=13 / \mathrm{N}=7 ; 1 / 3-\mathrm{DKO} n=13 / \mathrm{N}=10)$ and (B) $200 \mathrm{~ms}(\mathrm{Wt} n=12 / \mathrm{N}=8 ; 1 / 2-\mathrm{DKO} n=13 / \mathrm{N}=6 ; 1 / 3-\mathrm{DKO} n=14 / \mathrm{N}=10)$ depolarization stimuli.

C Average time of return to baseline obtained by fitting a linear function to the $C_{m}$ data following a 20-ms depolarization.

D Average slope of the linear component of endocytosis for 20-ms and 200-ms depolarizing pulses, obtained from fitting $C_{m}$ data for $20 \mathrm{~s}$ after cessation of the 20 ms depolarizations or for the last $10 \mathrm{~s}$ of the $C_{m}$ recording for 200 -ms depolarizations $\left({ }^{\star} P<0.05\right.$; one-way ANOVA with post hoc Tukey's or non-parametric K-W with post hoc Dunn's test).

E, F Average amplitude (E) and time constant (F) of the exponential component obtained from fits to $\mathrm{C}_{\mathrm{m}}$ data following $200-\mathrm{ms}$ depolarization $\left(n_{\text {exp fit }}=11\right.$ for Wt, 10 for $1 / 2-D K O$, and 11 for 1/3-DKO; $x$ data points in (E) correspond to IHCs with no exponential component in $C_{m}$ ). $\otimes$ corresponds to statistically identified outliers.

Data information: For panels (A, B), data represent grand averages \pm SEM; for ( $-F)$, box plots illustrate the median with the interquartile range, whiskers indicate 10 $90 \%$ of data points, and the squares present the respective mean value. Please also refer to Appendix Table S8 for detailed statistical analysis.

(Fig $8 \mathrm{~A}-\mathrm{D}$ ). Here, we observed an overall reduction of SVs at the AZs of $1 / 2$-DKO and 1/3-DKO IHCs. We further categorized SVs into ribbon-associated SVs (RA-SVs) and membrane-proximal SVs (MPSVs) (Fig 8B). The number of RA-SVs was significantly reduced in 1/2-DKO and 1/3-DKO IHCs compared to Wt (Fig 8C: one-way ANOVA, $F(3,417)=38.4, P<0.0001$; post hoc Tukey's analysis for all data sets can be found in Appendix Table S11) with the membrane-distal half of the ribbon being affected to a greater extent than the membrane-proximal half (Fig $8 \mathrm{C}^{\prime}: \quad \mathrm{KWS}=82.4$, $P<0.0001$; post hoc Dunn's analysis can be found in Appendix Table S11). Likewise, the number of MP-SVs was reduced in IHCs lacking endophilins-A1/A2 or endophilins-A1/A3 (Fig 8D: one-way ANOVA, $F(3,425)=17.6, P<0.0001$; post hoc Tukey's results for all data sets can be found in Appendix Table S11).

In order to further decipher the distribution of vesicles and ELVs at the ribbon in 3D, we additionally performed 3D electron tomography, which offers an improved axial resolution to revisit our analysis of $1 / 2$-DKO and $1 / 3$-DKO IHCs (Fig $8 \mathrm{E}-\mathrm{J}$ ). Here, the reconstruction of the electron tomograms (Fig 8E, an overview of the analysis parameters and criteria can be found in Fig $8 \mathrm{~F}$ ) corroborated the above notions and revealed a statistically significant reduction of RA-SVs (Fig 8G: one-way ANOVA, $F(2,21)=3.899, P=0.036$; post hoc Tukey's test: $P=0.023$ for $1 / 2$-DKO versus $W t$ and $P=0.14$ for 1/3-DKO versus Wt) and of MP-SVs (Fig 8H; one-way ANOVA, 

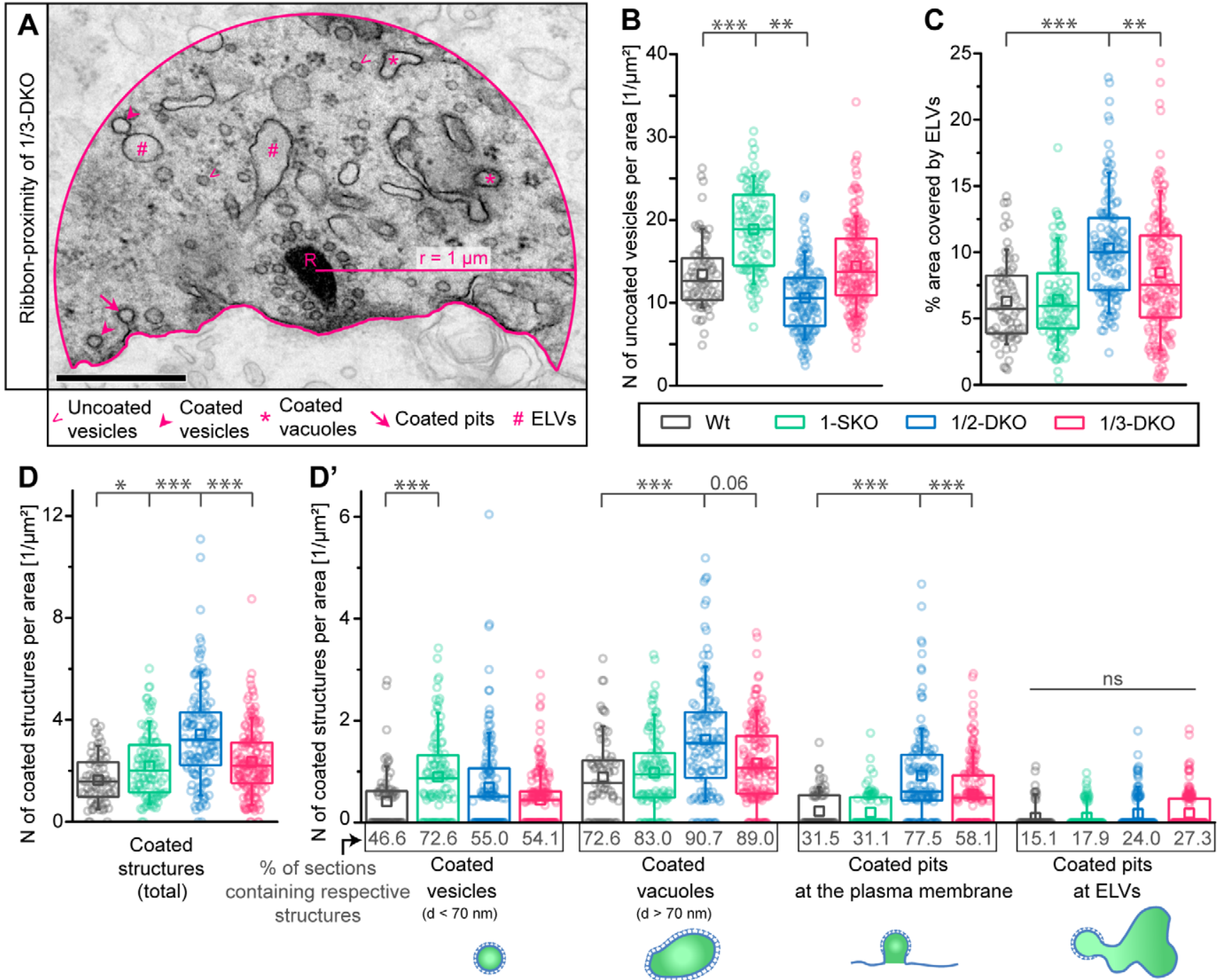

Figure 7. Decreased number of small vesicles, accumulations of coated structures and ELVs at IHC synapses of endophilin mutants.

A Representative electron micrograph illustrating the region of interest for analysis within a radius of $r=1 \mu \mathrm{m}$ from the ribbon center (exemplary for a 1/3-DKO specimen). Graphical aids indicate small vesicles, ELVs, and different subpopulations of coated structures quantified in (B-D). Scale bar: $500 \mathrm{~nm}$.

B While an increased number of small uncoated vesicles could be observed at 1-SKO AZs, reduced numbers of uncoated vesicles were present in a $1 \mu \mathrm{m}$ radius around the ribbon of 1/2-DKOs. RA-SVs and MP-SVs were excluded from this quantification.

C Accumulations of ELVs occurred in 1/2-DKO and 1/3-DKO IHCS, as measured by the relative cumulative ELV area per section.

D Increased overall number of coated structures in endophilin mutants. ( $\mathrm{D}^{\prime}$ ) Prominent accumulations of coated pits could be observed in 1/2-DKO and 1/3-DKO IHCS alongside a shift toward more coated vacuoles (d $>70 \mathrm{~nm}$ ) in 1/2-DKO IHCS and 1/3-DKO IHCS.

Data information: For panels (B-D), the following numbers of replicates were used: Wt $n=72 / \mathrm{N}=2 ; 1$-SKO $n=106 / \mathrm{N}=3 ; 1 / 2-\mathrm{DKO} n=129 / \mathrm{N}=3 ; 1 / 3$-DKO $n=172 /$ $N=6$. Box plots illustrate the median with the interquartile range, whiskers indicate 10-90\% of data points, and the squares present the respective mean value; $\mathrm{K}-\mathrm{W}$ followed by Dunn's test; ${ }^{*} P<0.05$; ${ }^{* *} P<0.01$; ${ }^{* \star *} P<0.001$. Please also refer to Appendix Tables $\$ 9$ and $\$ 10$ for detailed statistical analysis.

$F(2,21)=4.912, P=0.0178$; post hoc Tukey's test: $P=0.014$ for $1 /$ 2 -DKO versus $\mathrm{Wt}$ and $P=0.051$ for $1 / 3$-DKO versus $\mathrm{Wt}$ ) in $1 / 2$-DKO IHCs. The fraction of RA-SVs of the total count of SVs at the AZ was not significantly affected (one-way ANOVA; $F(2,21)=0.4416$, $P=0.6488$; see Appendix Fig S2B). Studying SV diameters with the greater precision of tomography, we found larger SVs at $1 / 2$-DKO AZs, but not at 1/3-DKO AZs (Fig 8I; one-way ANOVA, $F(2,21)=11.59, P=0.0004$; post hoc Tukey's test: $P=0.0215$ for $1 / 2$-DKO versus $\mathrm{Wt}$; $P=0.176$ for $1 / 3$-DKO versus $\mathrm{Wt}$; $P=0.0003$ for $1 / 2$-DKO versus $1 / 3$-DKO). Moreover, tomograms showed an increased abundance of ELVs at 1/2-DKO AZs and a non-significant trend for more ELVs at 1/3-DKO AZs (Fig 8J; one-way ANOVA, $F(2,21)=11.59, P=0.0004$; post hoc Tukey's test: $P=0.015$ for $1 /$ 2 -DKO versus $\mathrm{Wt}$; $P=0.136$ for $1 / 3$-DKO versus $\mathrm{Wt}$ ).

Finally, we examined the effects of potassium stimulation on the ultrastructure of endophilin-deficient IHCs. In these experiments, we subjected acutely dissected 1/2-DKOs-the genotype with the most perturbed presynaptic morphology - and Wt organs of Corti to a stimulatory solution ( $15 \mathrm{~min}, 50 \mathrm{mM} \mathrm{KCl}, 5 \mathrm{mM} \mathrm{CaCl}_{2}$ ) prior to immediate chemical fixation (Fig 9A). Analogous to the 

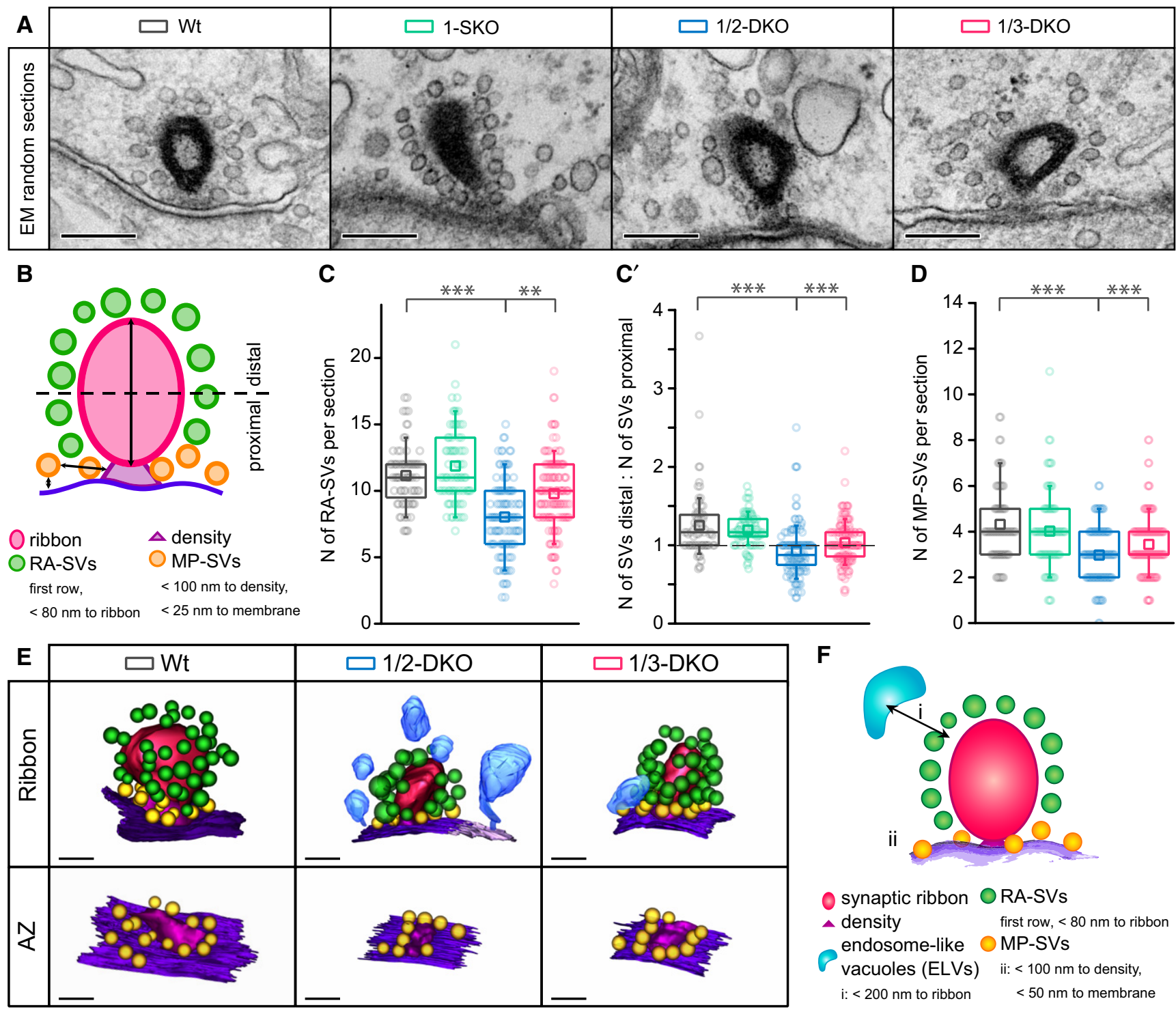

$\mathbf{F}$
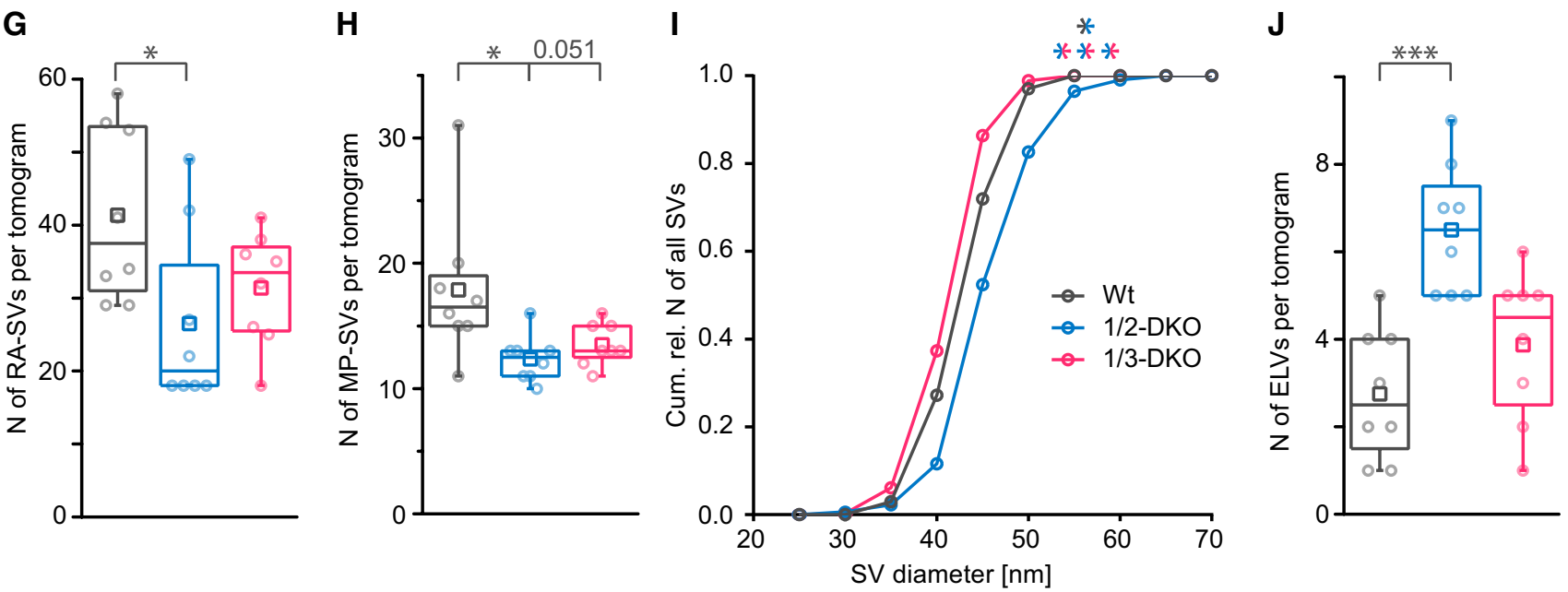

Figure 8. 
Figure 8. Absence of endophilins causes changes in SV number and distribution at IHC ribbon synapses.

A Representative electron micrographs of random ultrathin sections from IHC ribbon synapses of p15 mutants lacking different endophilin-A alleles. Scale bars: $200 \mathrm{~nm}$.

B Schematic illustration depicting the analysis criteria for random section electron micrographs (not drawn to scale). Synaptic vesicles (SVs) were categorized as ribbon-associated SVs (RA-SVs, green) or membrane-proximal SVs (MP-SVs, yellow).

C, D Reduced numbers of RA-SVs (C) and of MP-SVs (D) in IHCS of endophilin mutants with the strongest reduction observed in 1/2-DKO mice. One-way ANOVA followed by post hoc Tukey's test; ${ }^{* \star} P<0.01 ;{ }^{* \star *} P<0.001$

$\mathrm{C}^{\prime} \quad$ Reduced ratio of RA-SVs in the distal half of the ribbon over RA-SVs in its proximal half in 1/2-DKO and 1/3-DKO IHCS. K-W followed by Dunn's test; $\star \star \star P<0.001$.

E Representative 3D reconstructions of EM tomograms of 250-nm sections from Wt and endophilin DKO mutants. Please note the accumulations of ELVs in IHCs of endophilin 1/2-DKOs. Analysis was performed on eight tomograms from two animals per genotype. Scale bars: $100 \mathrm{~nm}$.

F Schematic illustration depicting the analysis criteria for tomograms (not drawn to scale). SVs were categorized as RA-SVs (green) and as MP-SVs (yellow). ELVs (light blue) were counted if the smallest distance between ELV and ribbon was $<200 \mathrm{~nm}$.

G Significantly reduced numbers of RA-SVs in 1/2-DKO IHCS (one-way ANOVA followed by post hoc Tukey's test; ${ }^{\star} P=0.0229$ ).

$\mathrm{H} \quad$ Significantly reduced numbers of MP-SVs in $1 / 2-\mathrm{DKO}$ and a strong trend $(P=0.0511$ ) toward less MP-SVs in $1 / 3-\mathrm{DKO}$ IHCS (one-way ANOVA followed by post hoc Tukey's test; $\left.{ }^{*} P=0.0138\right)$

Increased SV diameter in 1/2-DKOs, but unchanged SV diameter in 1/3-DKOs (one-way ANOVA followed by post hoc Tukey's test; ${ }^{\star} P=0.0154$ ).

J Accumulation of ELVs with a minimal distance to the ribbon of less than $200 \mathrm{~nm}$ in 1/2-DKOs (one-way ANOVA followed by post hoc Tukey's test; ${ }^{* \star \star} P=0.0002$ ).

Data information: Analysis was performed on random sections of ribbon-occupied AZs derived from several IHCS per genotype; the following numbers of replicates were used: Wt $n=78$ sections from $N=2$ animals; 1 -SKO $n=95, N=3 ; 1 / 2$-DKO $n=135, N=3 ; 1 / 3$-DKO $n=176, N=6$. Box plots illustrate the median with the interquartile range, whiskers indicate $10-90 \%$ of data points, and the squares present the respective mean value. For panels (C-E), please also refer to Appendix Tables S11 and S12 for detailed statistical analysis.

unstimulated probes, we compared SV pools in direct vicinity of the ribbon. Here, both, RA- and MP-SV numbers were significantly lower in stimulated 1/2-DKO compared to stimulated Wt (Fig 9B and C; Mann-Whitney test, for RA-SVs: $U=368, P<0.0001$; for MP-SVs: $U=783, P=0.0438$ ). Moreover, all findings made within the analyzed $1 \mu \mathrm{m}$ perimeter of the ribbon of unstimulated $1 / 2$-DKO IHCs compared to unstimulated Wt IHCs were also present in the stimulated IHCs of 1/2-DKO (compared to stimulated Wt IHCs), but to a greater extent. We found (i) reduced numbers of small uncoated cytosolic vesicles (Fig 9D; Student's $t$-test, $t=4.67$; $P<0.0001$ ) and (ii) vast accumulations of ELVs (Fig 9E; Mann-Whitney test, $U=264 ; P<0.0001)$. The formation of coated pits at ELVs was more frequently seen in stimulated $1 / 2$-DKO than in stimulated $\mathrm{Wt}$ or unstimulated samples (Fig 9A and $\mathrm{F}^{\prime}$; Mann-Whitney test, $U=623, P=0.001$; see also Appendix Table S13 for the comparisons of other coated structures), again pointing toward a function of endophilin in clathrin-dependent SV reformation from ELVs.

In summary, our electrophysiological and ultrastructural analyses indicate that loss of endophilins from IHCs impaired, but did not abolish, presynaptic endocytosis. Fission and uncoating of clathrincoated membranous structures, e.g., processes such as clathrindependent SV reformation, seem to be facilitated by-but do not essentially require - endophilins in IHCs.

\section{Discussion}

The presented morphological and physiological analysis of IHC ribbon synapses lacking endophilins provides novel insights into the molecular regulation of transmitter release and membrane retrieval in IHCs. Based on our findings, we propose that the main functions of the endophilin family at IHC synapses are to promote (i) the organization of $\mathrm{Ca}^{2+}$ channel clusters and presynaptic $\mathrm{Ca}^{2+}$ influx, (ii) replenishment of SVs to the release sites, (iii) coupling of exocytosis and endocytosis, probably via interaction with otoferlin, (iv) endocytic membrane retrieval, and (v) SV reformation from clathrincoated structures (summarized in the cartoon in Fig 10).

\section{Endophilin-A1 positively regulates the abundance and modulates the function of Cav1.3 channels at IHC synapses}

While endophilins are indispensable for life, they do not seem to be required for the development and survival of the organ of Corti, even though single-cell RT-PCR indicated the expression of all three endophilins in IHCs. Given the perinatal lethality of endophilin TKO mice, we analyzed their IHCs in organotypic cultures, which did not show obvious developmental or gross morphological deficits. They did, however, show a reduction of depolarization-evoked $\mathrm{Ca}^{2+}$ currents and enhanced inactivation kinetics (in the TKO), features that could partly also be recapitulated in the viable 1 -SKOs and $1 / 3$ DKO mice after hearing onset. Moreover, STED microscopy of $\mathrm{Ca}_{\mathrm{V}} 1.3$ immunofluorescence indicated a reduction in $\mathrm{Ca}^{2+}$ channel cluster size at the presynaptic AZ membrane of endophilin mutants. These observations provide insights into the functional consequences of the interaction of endophilins with $\mathrm{Ca}^{2+}$ channels that had previously been reported by biochemistry (Chen et al, 2003). To our knowledge, this is the first report showing such a positive regulation of $\mathrm{Ca}^{2+}$ channel abundance and function (i.e., inhibiting inactivation) by endophilins. The $\mathrm{Ca}^{2+}$ current inactivation phenotype we observed in organotypically cultured IHCs of TKO mice appeared to be compensated in the other mutants studied-likely by expression of the remaining endophilins-and hence seems to require the complete loss of all endophilins to become apparent. Notably, the observed decrease in whole-cell $\mathrm{I}_{\mathrm{Ca}}$ was not accompanied by (i) detachment of synaptic ribbons, (ii) loss of afferent synapses, or (iii) mislocalization of presynaptic $\mathrm{Ca}_{\mathrm{V}} 1.3 \mathrm{Ca}^{2+}$ channels. Indeed, the channels remained tightly clustered in the AZ membrane underneath the normally sized synaptic ribbon, as shown by super-resolution microscopy. Instead, our data reflect a reduced presynaptic abundance of $\mathrm{Ca}_{V} 1.3 \mathrm{Ca}^{2+}$ channels, as demonstrated by the decreased size of presynaptic $\mathrm{Ca}^{2+}$ channel clusters of 1-SKOs and 1/3-DKOs. Future studies should test how endophilins promote a large $\mathrm{Ca}^{2+}$ channel complement at the IHC AZ and whether such positive regulation of $\mathrm{Ca}^{2+}$ channel abundance is found also at other synapses. 

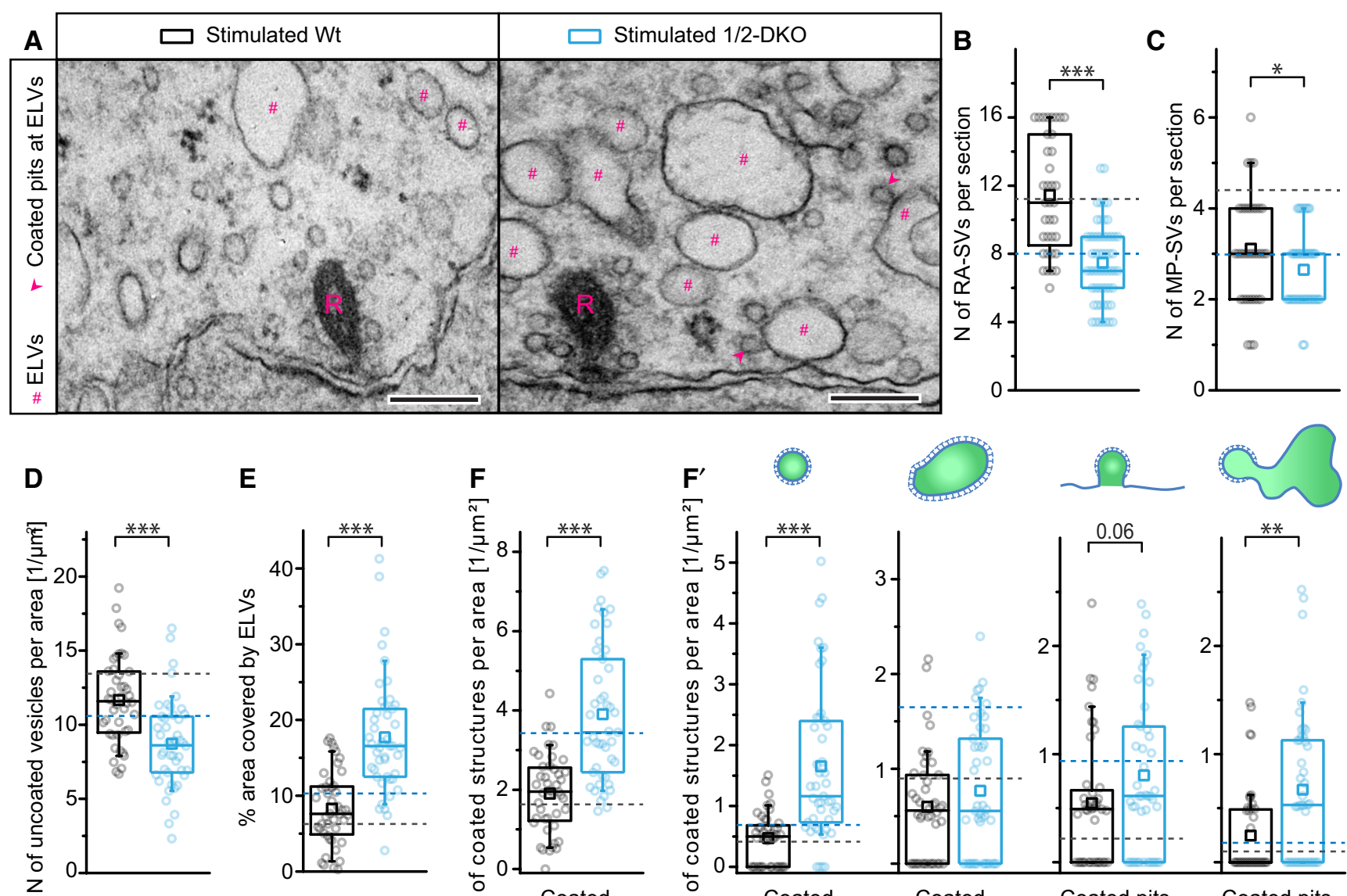

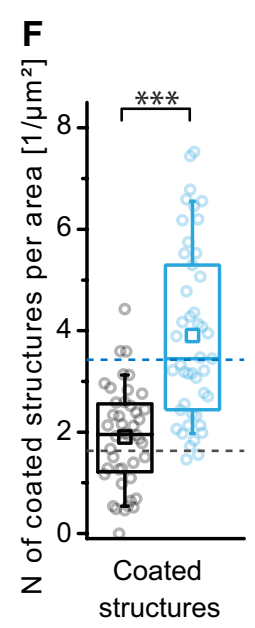

(total)

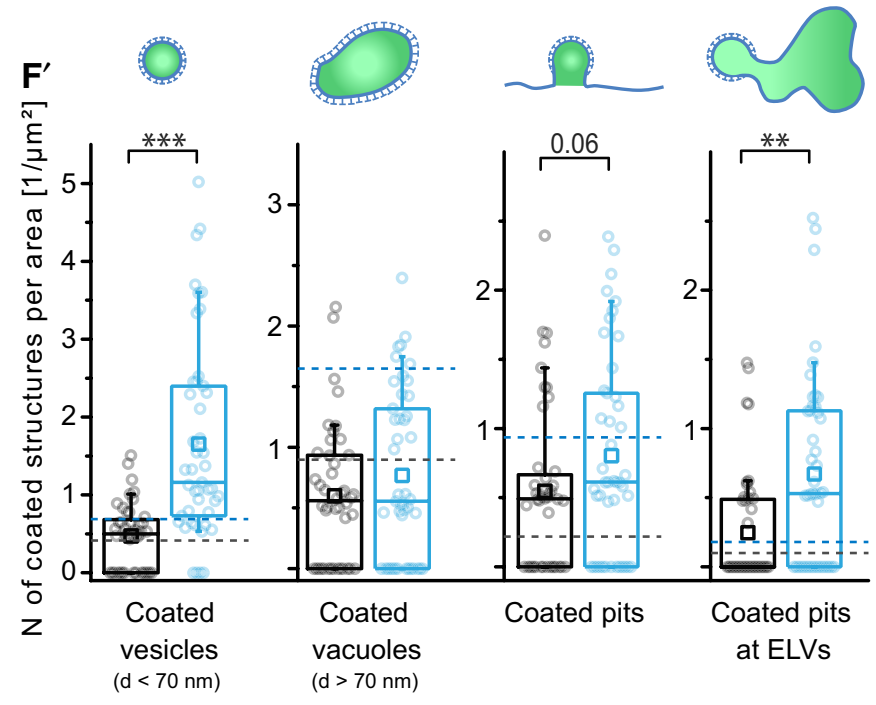

Figure 9. High $\mathrm{K}^{+}$stimulation leads to accumulations of ELVs and an increased occurrence of coated pits at ELVs in endophilin 1/2-DKOs.

A Representative electron micrographs of Wt and 1/2-DKO specimen indicate accumulations of ELVs and high numbers of coated pits forming from ELVs in ribbon

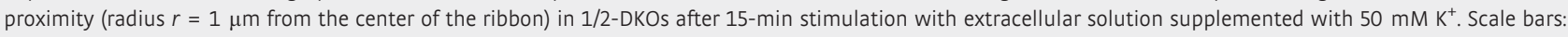
$200 \mathrm{~nm}$.

B Significantly reduced numbers of RA-SVs in IHCS of 1/2-DKO compared to Wt after stimulation, as quantified using random ultrathin sections of ribbon-occupied AZs from several IHCS per genotype. Mann-Whitney test; ${ }^{* *} P<0.001$

C Numbers of MP-SVs were slightly reduced in stimulated 1/2-DKOs compared to stimulated Wt. Mann-Whitney test; ${ }^{*} P<0.05$.

D Numbers of small uncoated vesicles were reduced in a $1 \mu \mathrm{m}$ radius around the ribbon of 1/2-DKOs compared to Wt after stimulation. RA-SVs and MP-SVs were excluded from this quantification. Student's $t$-test; ${ }^{* \star} p<0.001$.

E Stimulated 1/2-DKOs exhibited accumulations of ELVs in comparison with stimulated Wt. Mann-Whitney test; *** $<0.001$

F Increased overall number of coated structures in stimulated endophilin 1/2-DKOs compared to stimulated Wt. (F') Both, coated vesicles and coated pits forming at ELVs, were significantly increased in 1/2-DKOs after stimulation. Mann-Whitney test; ${ }^{\star \star} P<0.01$; ${ }^{* \star *} P<0.001$.

Data information: Stimulated Wt $n=36$ sections from $N=2$ animals; stimulated 1/2-DKO $n=76 / N=3$; dashed lines indicate mean values of the unstimulated conditions from the respective genotypes for reference. Box plots illustrate the median with the interquartile range, whiskers indicate $10-90 \%$ of data points, and the squares present the respective mean value. For panels (B-F), please also refer to Appendix Table S13 for detailed statistical analysis.

\section{Endophilins facilitate endocytic membrane retrieval and reformation of SVs}

Our focus on mature IHCs, moving away from cultured TKO cells, leaves room for functional compensation given that endophilins have previously been shown to exhibit redundancy for several cellular functions (Milosevic et al, 2011; Murdoch et al, 2016). Nonetheless, we found a modest accumulation of clathrin-coated pits (CCPs) in the proximity of IHC ribbon-type AZs, suggesting that even partial absence of endophilins becomes rate-limiting for the fission process at
IHC synapses. Indeed, this hypothesis is strongly supported by our finding of a reduced rate in the slow (linear) component of endocytic membrane retrieval in 1/2-DKO and 1/3-DKO IHCs that has previously been attributed to CME (Neef et al, 2014). In neurons, endophilin is recruited to CCPs prior to membrane fission (Ringstad et al, 1999; Perera et al, 2006; Ferguson et al, 2009; Milosevic et al, 2011; Sundborger et al, 2011), and several other studies have reported an accumulation of CCPs following perturbation of endophilin function (Ringstad et al, 1999; Gad et al, 2000; Schuske et al, 2003; Verstreken et al, 2003; Sundborger et al, 2011). Yet, no significant 


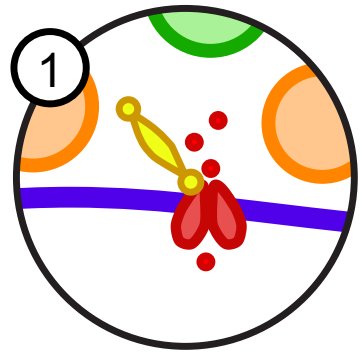

$\mathrm{Ca}_{\mathrm{v}} 1.3$ functional modulation reductions of $\mathrm{Ca}^{2+}$ influx alongside a reduced size but preserved shape of $\mathrm{Ca}_{\vee} 1.3$ clusters

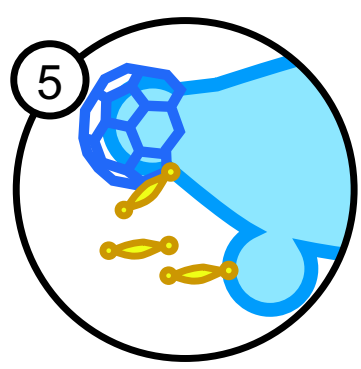

SV formation from ELVs

reduced SV numbers, ELV accumulations and reduction of sustained exocytosis beyond what is expected based on lowered $\mathrm{Ca}^{2+}$ influx 3

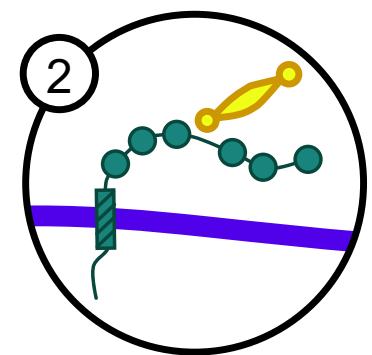

Coupling of exo- and endocytosis direct interaction of endophilin and otoferlin

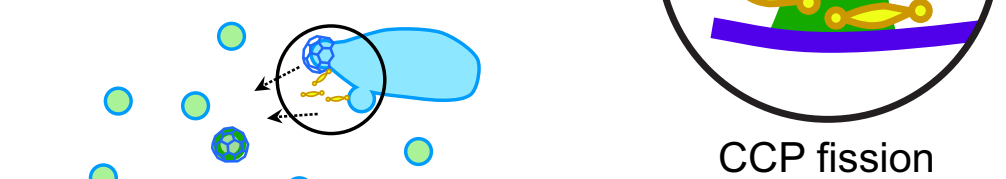

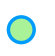

0
O

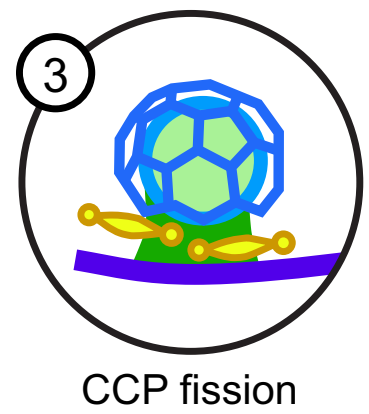

increased numbers of coated pits and slower rates of $\mathrm{IHC}$ endocytic membrane retrieval
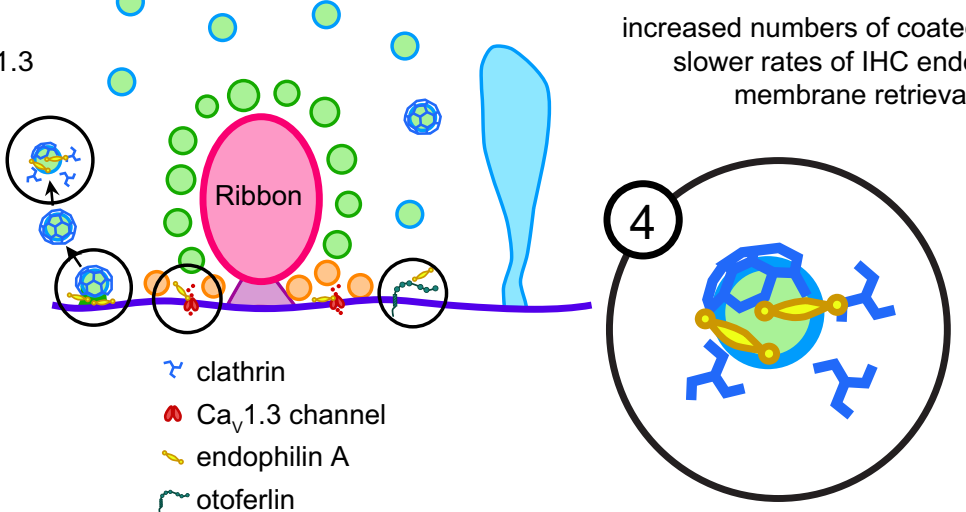

SV uncoating

accumulations of coated structures in proximity of the ribbon

Figure 10. Putative functions of endophilins in IHCs.

All three endophilin-A genes are present in IHCs. We found two novel functions of endophilin-A1: a positive regulation of IHC presynaptic Ca ${ }^{2+}$ influx and Ca ${ }^{2+}$ channel abundance (1) and a physical interaction of endophilin-A1 with the exocytic protein otoferlin that might contribute to exocytosis-endocytosis coupling (2). Moreover, like in neurons, all endophilins in IHCs seem to be involved in fission (3) and uncoating (4) of clathrin-coated organelles supporting CME, and, furthermore, promote SV reformation from endosomal intermediates (5).

accumulation of CCPs was detected at cortical synapses at rest in endophilin 1/2-DKO and TKO mice (Milosevic et al, 2011), suggesting that fission was not rate-limiting in this model. Given that endophilins interact directly with dynamin 1 (Ringstad et al, 1999; Anggono \& Robinson, 2007), they likely promote dynamin's recruitment to the neck of CCPs in IHCs, as they do in neurons (Perera et al, 2006; Ferguson et al, 2009; Milosevic et al, 2011). Furthermore, endophilins have been reported to have a direct role in dynamin-independent scission of tubular membrane necks in vitro (Renard et al, 2015; Simunovic et al, 2017). It is therefore likely that endophilin promotes fission in IHCs via recruitment of dynamins; however, a dynamin-independent role of endophilin in fission remains possible.

Not only the higher numbers of coated pits but also larger coated vacuoles in IHCs of both endophilin DKOs may serve as an indicator for the impaired fission. Alternatively or in addition, endophilin may, as previously reported in invertebrates (Bai et al, 2010), be required for membrane bending prior to the pit formation, thus resulting in larger coated structures. Curiously, the number of coated vesicles was significantly increased in 1-SKO IHCs, but not in 1/2-DKO or 1/3-DKO IHCs, which have an overall much stronger phenotype. Yet, if the numbers of coated vesicles and coated vacuoles are pulled together, one can notice a shift toward larger coated structures in the endophilin 1/2-DKO and 1/3-DKO IHCs. One possible explanation here is that the recruitment of dynamin(s) and/or the fission process may not be rate-limiting in the absence of endophilin-A1 alone. The recruitment of synaptojanin-1 and/or other factors involved in uncoating is already disturbed in synapses of endophilin A1-SKOs, though. This finding is in agreement with Milosevic et al (2011) who reported accumulations of CCVs at murine cortical synapses of $1 / 2$-DKO and TKO mice.

In addition to the increased occurrence of coated pits and coated vesicles in the proximity of IHC ribbon synapses, we observed a 
prominent accumulation of other recycling intermediates with a clathrin coat. Most strikingly in stimulated 1/2-DKO IHCs, coated pits accumulated at ELVs, indicating that fission also displays the rate-limiting step here. Consistent with a role of endophilins in SV reformation, we further found an increased occurrence of ELVs at endophilin 1/2-DKO mutant AZs and a larger area covered by ELVs in both DKO IHCs. Similar accumulation of ELVs can also be found in AP-2-deficient IHCs, which however, unlike endophilin mutants, showed a clear reduction of coated structures in the ribbon's vicinity (Jung et al, 2015). One likely hypothesis is that clathrin recruitment is disturbed in AP-2 mutants, whereas in endophilin mutants, fission and/or uncoating limits endocytic membrane retrieval and SV reformation.

Future studies should address to which extent the impaired SV reformation contributes to the reduction of vesicle replenishment during sustained exocytosis observed in our $\mathrm{C}_{\mathrm{m}}$ recordings in endophilin-deficient IHCs (see below). Importantly, while a prominent reduction in the number of SVs and protein levels of several main SV proteins had been observed at cortical synapses, we detected only mild-albeit statistically significant-alterations in vesicle numbers of endophilin mutants (as reported in Figs 7 and 8). It is not trivial to test the levels and distribution of proteins in this system, in part since IHCs do not use the same molecular machinery as cortical neurons: for example, (i) IHC exocytosis operates without classical neuronal SNAREs including synaptobrevin-2 (Nouvian et al, 2011) and (ii) since various antibodies against SV proteins are found to be non-specific in our hands in IHCs (e.g., synaptojanin-1), thus limiting the tools available to examine SV proteins in IHCs. Nevertheless, while we observed altered levels/distribution of otoferlin and $\mathrm{Ca}^{2+}$ channels, we did not detect obvious differences in the levels and/or distribution of AP-2 or myosin 6, which has previously been reported to be required for ribbon synapse maturation and function (Roux et al, 2009), or AP-2.

\section{Endophilins are involved in SV replenishment at IHC AZs}

Double and triple mutants of endophilins showed reduced sustained exocytosis, likely caused by impaired recruitment of SVs to the release sites of the IHC AZ. It is unlikely that the deficits in sustained exocytosis solely reflect the slowed reformation of SVs (see above), as the overall numbers of small, uncoated vesicles were unaltered in 1/3-DKOs. Here, to our knowledge for the first time, we describe an interaction of endophilin-A1 with otoferlin, a critical component in the processes of synaptic release and SV replenishment in IHCs (Roux et al, 2006; Pangrsic et al, 2010; Vogl et al, 2016). This interaction appears especially relevant in light of the previously described observations. In fact, in mouse mutants suffering from reduced otoferlin levels, such as the otoferlin mutants pachanga (Pangrsic et al, 2010) or otoferlin Ile515Thr (Strenzke et al, 2016), as well as a knockout mouse model for AP- $2 \mu$ (Jung et al, 2015), sustained exocytosis is likewise disturbed.

However, the mechanism at work here seems to be different from what was observed in AP- $2 \mu \mathrm{KO}$. While endophilin-A1, like AP-2, is an interaction partner of otoferlin (Duncker et al, 2013; Jung et al, 2015), lack of endophilin does not cause a drastic decrease in IHC otoferlin levels or alter the subcellular distribution of otoferlin, as seen in AP- $2 \mu$-deficient IHCs. In fact, genetic loss of $\mathrm{AP}-2 \mu$ results in dramatically decreased otoferlin expression and a significant reduction in sustained exocytosis, as well as a profound hearing loss, as assessed by ABR recordings. Importantly, AAVmediated AP- $2 \mu$ rescue succeeded in restoring otoferlin levels of AP$2 \mu$ mutant IHCs to $\sim 50 \%$ of Wt levels, which sufficed to fully restore the observed exocytosis phenotype and drastically improved ABR performance. Based on these findings, we would suspect that the overall reduction of $\sim 25 \%$ of otoferlin levels in endophilin 1/3-DKO should not have any major effects on IHC exocytic performance in the endophilin mutants. Therefore, we propose that the impairment of sustained exocytosis is not due to the reduction of otoferlin levels. The precise mechanism(s) by which endophilins promote replenishment of SVs to the release site remain to be investigated in future studies. The reduced number of RA-SVs at endophilin-deficient AZs might indicate that endophilins are required for efficient resupply of SVs to the ribbon. Given that endophilins are capable of interacting with actin and/or actin-modifying proteins such as intersectin (Ferguson et al, 2009; Soda et al, 2012; Vehlow et al, 2013; Pechstein et al, 2015; Yang et al, 2015), as well as that the F-actin cytoskeleton is important for SV exocytosis in IHCs (Vincent et al, 2015; Guillet et al, 2016), an additional role of endophilin in SV resupply to the ribbon appears likely. Alternatively or in addition, endophilin binding to otoferlin at the release site might facilitate the lateral diffusion of fused vesicular proteolipids (site clearance) as proposed for AP-2 (Jung et al, 2015).

\section{Materials and Methods}

\section{Animals}

All experiments complied with national animal care guidelines and were approved by the University Medical Center Göttingen Board for animal welfare and the animal welfare office of the state of Lower Saxony. Constitutive knockout mice for endophilin-A1 $\left(E 1^{-/-}\right)$, endophilin-A2 $\left(E 2^{-/-}\right)$, endophilin-A3 $\left(\mathrm{E}^{-/-}\right)$, described in Milosevic et al (2011), were employed in two separate breeding schemes: (i) perinatally lethal $\mathrm{E} 1^{-/-} \mathrm{E} 2^{-/}-\mathrm{E} 3^{-/-}$(hereafter dubbed TKO) mice, as well as viable $\mathrm{E} 1^{-/-} \mathrm{E} 2^{+/-} \mathrm{E} 3^{-/-}$and $\mathrm{E} 1^{-/-} \mathrm{E} 2^{+/}$ ${ }^{+} \mathrm{E} 3^{-/-}$mice (hereafter pooled, as we did not find significant differences in IHC physiology and morphology and dubbed 1/3DKOs), were obtained from breeding $\mathrm{E} 1^{-/-} \mathrm{E} 2^{+/-} \mathrm{E} 3^{-/-}$mice and (ii) mating of $\mathrm{E} 1^{-/-} \mathrm{E} 2^{+/-}$mice yielded $\mathrm{E} 1^{-/-} \mathrm{E} 2^{-/-}$mice (hereafter dubbed 1/2-DKOs), and $\mathrm{E} 1^{-/-} \mathrm{E} 2^{+/-}$and $\mathrm{E} 1^{-/-} \mathrm{E} 2^{+/+}$(hereafter pooled and dubbed 1-SKOs). $\mathrm{E} 1^{+/+} \mathrm{E} 2^{+/+} \mathrm{E} 3^{+/+}$with the same genetic background (approximately 80\% C57BL/6J $+20 \%$ SV129) were bred to generate wild-type controls (Wt) for electron microscopy, immunohistochemistry, and physiology experiments. For gene expression studies and a set of cell physiology experiments, we employed C57BL/6J mice as Wt controls. Most experiments were performed at 2-3 weeks of age (after hearing onset around postnatal day p12 in mice; Mikaelian \& Ruben, 1965), except for (i) cell physiology on TKO mice, which due to perinatal lethality were used within hours after birth to prepare organotypic cultures of organs of Corti, and (ii) auditory brainstem responses that were recorded at 6-8 weeks (taking into consideration that the C57BL/6J background is genetically predisposed for early onset age-related hearing loss; Shnerson \& Pujol, 1981). Both male and female mice were used for all experimental paradigms. 


\section{Single-cell RT-PCR}

To determine the expression of the three endophilin- $A$ genes in IHCs, we isolated mRNA from single IHCs of C57BL/6J mice at p14-16. In these experiments, individual IHCs were harvested from the apical coils of freshly dissected organs of Corti after cleaning off supporting cells. The filtered bath solution contained (in mM) $5.36 \mathrm{KCl}, 141 \mathrm{NaCl}, 0.5 \mathrm{MgSO}_{4} \cdot 7 \mathrm{H}_{2} \mathrm{O}, 10$ HEPES, $1 \mathrm{MgCl}_{2}, 1.3$ $\mathrm{CaCl}_{2}(\mathrm{pH} 7.2, \sim 300 \mathrm{mOsm} / \mathrm{l})$ and was continuously perfused at high rate $(1.7-3.3 \mathrm{ml} / \mathrm{min})$ to clear off cell debris. Individual IHCs were aspirated into a glass pipette containing $8 \mu \mathrm{l}$ of intracellular solution ( $135 \mathrm{mM} \mathrm{KCl,} 10 \mathrm{mM}$ HEPES, $0.5 \mathrm{mM} \mathrm{MgCl}_{2} \cdot 6 \mathrm{H}_{2} \mathrm{O}$ ). The pipette content was then transferred into first-strand cDNA synthesis mix containing after dilution (in $\mathrm{mM}$ ): 50 Tris- $\mathrm{HCl}$ ( $\mathrm{pH} 8.4$ ), 50 $\mathrm{KCl}, 5 \mathrm{MgCl}_{2}, 10 \mathrm{DTT}$, and 50 units of SuperScript II Reverse Transcriptase (Invitrogen, Carlsbad, CA) and 40 units of RNaseOUT ribonuclease inhibitor (Invitrogen). Aspirated bath solution in close proximity to the IHCs before and after IHC harvesting was used as a negative control. Reverse transcription (RT) was performed with SuperScript ${ }^{\mathrm{TM}}$ II RT and SuperScript ${ }^{\circledR}$ First-Strand Synthesis System for RT-PCR according to the manufacturer's instructions using oligo(dT) primers and random hexamers (Invitrogen). After ethanol precipitation, cDNA was pre-amplified with 20 cycles of a multiplex PCR, and the reaction product was used to perform quantitative real-time polymerase chain reaction (qPCR) using SYBR $^{\circledR}$ Green (Thermo Fisher). Sequences of endophilin-A-specific primers are listed in Appendix Table S1. Each sample was processed as technical duplicates using QuantStudio ${ }^{\mathrm{TM}} 6$ Flex (Life Technologies). Data were analyzed with QuantStudio ${ }^{\text {TM }}$ Real-Time PCR software.

\section{Immunoblotting of cochlear extracts}

Detection of endophilin-A1-3 protein from cochlear lysates was performed by Western blotting essentially as described in Vogl et al (2017). In brief, p15 animals of all respective genotypes were sacrificed and cochleae, hippocampi, and cerebellar fragments were dissected in ice-cold PBS. Additionally, modioli and organs of Corti were carefully micro-dissected and respectively pooled from $10 \mathrm{Wt}$ animals to assess expression in the two tissue fractions. Pooled cochleae of individual animals as well as the selected other tissue samples were transferred to fresh tubes containing a modified RIPA lysis buffer with the following composition (in mM): Tris- $\mathrm{HCl}(\mathrm{pH}$ 7.5) (50), $\mathrm{NaCl}$ (150), Na-deoxycholate (1), EDTA (1), PMSF (1), NaF (1), 1\% IGEPAL (v/v), 0.25\% (w/v), PhosSTOP phosphatase inhibitors, and complete protease inhibitors (Roche Holding AG, Basel, Switzerland). After manual homogenization of the tissue with a Teflon plunger and initial incubation for $30 \mathrm{~min}$ on ice, samples were transferred to a rotating wheel and gently lysed for an additional $2.5 \mathrm{~h}$ at $4^{\circ} \mathrm{C}$. Subsequently, samples were centrifuged at $1,000 \mathrm{~g}$ for $15 \mathrm{~min}$ at $4^{\circ} \mathrm{C}$ to precipitate large cell debris and bone fragments. Thereafter, the protein concentrations of the sample supernatants were determined using a BCA protein determination assay (Thermo Fisher) and the remaining samples were boiled for $6 \mathrm{~min}$ at $95^{\circ} \mathrm{C}$ in SDS sample buffer $(62.5 \mathrm{mM}$ Tris- $\mathrm{HCl}$ ( $\mathrm{pH} \mathrm{6.8),}$ $10 \%$ (v/v) glycerin, $2 \%$ SDS (w/v), $5 \%$ (v/v) $\beta$-mercaptoethanol, $0.002 \%(\mathrm{w} / \mathrm{v})$ brome phenol blue). Then, $10 \mu \mathrm{g}$ of protein per sample was subjected to $12 \%$ SDS-PAGE, transferred onto PVDF membranes, and-after blocking with $5 \%$ (w/v) milk powder dissolved in TBS-Tween-probed with the following primary antibodies: goat anti-endophilin-A1 (sc-10875; Santa Cruz), rabbit antiendophilin-A2 (a kind gift of Pietro de Camilli, Yale University, USA), goat anti-endophilin-A3 (sc-10880; Santa Cruz or Abcam, ab184008), and mouse anti- $\gamma$-adaptin (Cat. No.: 610386; BD Transduction Laboratories). After extensive washing, species-specific peroxidase-conjugated secondary antibodies were applied for chemiluminescent detection on an Intas ChemoCam imaging platform. In our experiments, none of the tested A3 antibodies showed selectivity for A3 but rather appeared to (also) detect A1.

\section{Immunohistochemistry and confocal microscopy of immunolabeled hair cells}

Freshly dissected apical cochlear turns of 2-week-old mice were fixed with (i) $4 \%$ formaldehyde (FA) in phosphate-buffered saline (PBS) - depending on the experimental paradigm either for 20 or for $60 \mathrm{~min}$-on ice, or (ii) for $20 \mathrm{~min}$ in methanol at $-20^{\circ} \mathrm{C}$ ( $\mathrm{Ca}_{\mathrm{V}} 1.3$-staining). Thereafter, specimens were washed in PBS and incubated for $1 \mathrm{~h}$ in goat serum dilution buffer (GSDB: 16\% normal goat serum, $450 \mathrm{mM} \mathrm{NaCl}, 0.3 \%$ Triton X-100, $20 \mathrm{mM}$ phosphate buffer, $\mathrm{pH}$ 7.4) in a wet chamber at room temperature. Primary antibodies were dissolved in GSDB and applied for $3 \mathrm{~h}$ at room temperature $\left(\mathrm{Ca}_{V} 1.3\right.$-staining), or overnight at $4^{\circ} \mathrm{C}$ in a wet chamber. After washing $3 \times 10 \mathrm{~min}$ (wash buffer: $450 \mathrm{mM} \mathrm{NaCl}, 20 \mathrm{mM}$ phosphate buffer, $0.3 \%$ Triton X-100), the tissue was incubated with GSDB-diluted secondary antibodies in a light-protected wet chamber for $1 \mathrm{~h}$ at room temperature. Then, the specimens were washed in wash buffer and finally in $5 \mathrm{mM}$ phosphate buffer and mounted onto glass microscope slides with mounting medium (Mowiol). The following primary antibodies were used: mouse monoclonal anti-CtBP2 (also recognizing the ribbon protein RIBEYE, 1:200, BD Biosciences, Cat. No. 612044), mouse antiotoferlin (1:300, Abcam, Cat. No. ab53233), rabbit anti-vGlut3 (1:300, Synaptic Systems, Cat. No. 135 203), rabbit anti-Ca 1.3 (1:30, Alomone Labs, Cat. No. ACC 005), and rabbit polyclonal myosin 6 (1:300, Proteus Biosciences Inc., \#25-6791). The secondary antibodies used for (i) confocal microscopy were goat anti-rabbit Alexa Fluor 488 and goat anti-mouse Alexa Fluor 568 (1:200, Invitrogen, Cat. No. A 11008 and A 11004, respectively), and (ii) STED were STAR 580-tagged goat anti-mouse (1:200, Abberior, Cat. No. 2-0002-005-1) and STAR 635P-tagged goat anti-rabbit (1:200, Abberior, Cat. No. 2-0012-007-2). Confocal images were acquired using a laser scanning confocal microscope (Leica TCS SP5, Leica Microsystems GmbH, Mannheim, Germany, and Zeiss LSM800, Carl Zeiss AG, Oberkochen, Germany) with $488 \mathrm{~nm}$ (Ar) and $561 \mathrm{~nm}$ (He-Ne) lasers for excitation and $1.4 \mathrm{NA} 63 \times$ oil immersion objectives. Z-axis stacks from comparable tonotopic regions were acquired with a pixel size of $80 \times 80 \mathrm{~nm}$ and step size of (i) $0.6 \mu \mathrm{m}$ for otoferlin quantifications and (ii) $0.25 \mu \mathrm{m}$ for ribbon counting. 2D STED images were acquired with a pixel size of $15 \times 15 \mathrm{~nm}$ using an Abberior Instruments Expert Line $775 \mathrm{~nm}$ 2-color STED microscope (Abberior Instruments, Göttingen, Germany), with excitation lasers at 561 and $640 \mathrm{~nm}$ and a STED laser at $775 \mathrm{~nm}, 1.2 \mathrm{~W}$, using a $1.4 \mathrm{NA} 100 \times$ oil immersion objective. Images were processed using ImageJ (http://imagej.net/) and assembled for display in Adobe Illustrator software. 


\section{Patch clamp of IHCs from cultured neonatal organs of Corti}

Organotypic cultures of perinatal (p0) mice were prepared as described previously (Nouvian et al, 2011; Reisinger et al, 2011; Vogl et al, 2015). Briefly, organs of Corti were dissected from TKO, 1/3-DKO, or C57BL/6J mice in HEPES-HBSS supplemented with $250 \mathrm{ng} / \mathrm{ml}$ fungizone (Life Technologies) and $10 \mu \mathrm{g} / \mathrm{ml}$ Penicillin G (Sigma-Aldrich), mounted on Cell-Tak ${ }^{\mathrm{TM}}$-coated coverslips (BD Biosciences) and incubated in DMEM/F12 with 5\% FBS. During the culture period of 1 week, half of the medium was replaced with fresh culture medium every second day. After 7 days in culture, perforated patch-clamp recordings were performed on apical coil IHCs using an extracellular solution containing (in mM) $103 \mathrm{NaCl}$, $2.8 \mathrm{KCl}, 1 \mathrm{MgCl}_{2}, 10$ HEPES, 35 TEA-Cl, $11.2 \mathrm{D}$-glucose, $10 \mathrm{CaCl}_{2}$, and apamin $(100 \mathrm{nM})$ to inhibit small conductance (SK) $\mathrm{K}^{+}$channels ( $\mathrm{pH} 7.2, \sim 300 \mathrm{mOsm} / \mathrm{l}$ ), and an intracellular solution containing (in mM) 129 Cs-gluconate, 10 TEA-Cl, 10 4-AP, 10 HEPES, 1 $\mathrm{MgCl}_{2}$, and amphotericin B $(300 \mu \mathrm{g} / \mathrm{ml})(\mathrm{pH} 7.2, \sim 280 \mathrm{mOsm} / \mathrm{l})$. In a subset of experiments, the extracellular $\mathrm{Ca}^{2+}$ concentration $\left(\left[\mathrm{Ca}^{2+}\right]_{\mathrm{e}}\right)$ was lowered to $6 \mathrm{mM}$ to reduce presynaptic $\mathrm{Ca}^{2+}$ influx in C57BL/6J mice; this was balanced by a complementary increase in $\mathrm{NaCl}$ to maintain overall ionic strength. All experiments were done at room temperature $\left(22-24^{\circ} \mathrm{C}\right)$ using an EPC10 amplifier with PatchMaster software (HEKA, Elektronik, Lambrecht, Germany). $\mathrm{Ca}^{2+}$ current and membrane capacitance measurements were performed using the Lindau-Neher technique (Lindau \& Neher, 1988; Moser \& Beutner, 2000). Currents were leak-subtracted with a $\mathrm{p} / 10$ protocol. Liquid junction potentials $(17 \mathrm{mV})$ were corrected offline.

\section{Patch clamp of IHCs in acutely dissected organs of Corti}

Patch-clamp recordings from IHCs of freshly dissected organs of Corti (apical coil) from p14-18 mice were essentially performed as described previously (Moser \& Beutner, 2000). For recordings of $\mathrm{Ca}^{2+}$ current, exocytosis, and endocytosis, the pipette solution contained (in mM) 129 Cs-gluconate, 10 TEA-Cl, 10 4-AP, 10 HEPES, $1 \mathrm{MgCl}_{2}$, and amphotericin B $(300 \mu \mathrm{g} / \mathrm{ml})$ (pH 7.2, $\sim 280 \mathrm{mOsm} / \mathrm{l}$ ). The extracellular solution contained (in $\mathrm{mM}$ ) 105 $\mathrm{NaCl}, 35 \mathrm{TEA}-\mathrm{Cl}, 2.8 \mathrm{KCl}, 2 \mathrm{CaCl}_{2}, 1 \mathrm{MgCl}_{2}, 5$ 4-AP, $1 \mathrm{CsCl}, 10$ HEPES, and 11.1 D-glucose (pH 7.2, 300 mOsm/l). EPC-9 amplifiers (HEKA) controlled by Pulse software (HEKA) were used for measurements. All voltages were corrected offline for the liquid junction potential $(14 \mathrm{mV})$. Currents were leak-corrected using a p/ 10 protocol for exocytosis or a p/20 protocol for endocytosis. Cells with leak currents exceeding $-35 \mathrm{pA}$ at $-84 \mathrm{mV}$ holding potential or with a series resistance higher than $30 \mathrm{M} \Omega$ were excluded from the analysis. Current-voltage (IV) relationships were obtained by applying 10-ms pulses of increasing voltage. For capacitance $\left(\mathrm{C}_{\mathrm{m}}\right)$ measurements, IHCs were stimulated by depolarizations of different durations to $-14 \mathrm{mV}$ at intervals of $20-120 \mathrm{~s}$.

\section{Electron microscopy and tomography}

Conventionally embedded samples for electron microscopy and tomography were prepared as described previously (Jung et al, 2015). Apical turns of the organ of Corti were acutely dissected and either stimulated for $15 \mathrm{~min}$ at room temperature with a solution containing (in mM) $50 \mathrm{KCl}, 95 \mathrm{NaCl}, 1 \mathrm{MgCl}_{2}, 5 \mathrm{CaCl}_{2}, 10$ HEPES and subsequently fixed for $1 \mathrm{~h}$ on ice with $4 \%$ PFA and $0.5 \%$ glutaraldehyde in PBS, or immediately fixed for $1 \mathrm{~h}$ on ice with the same fixative. Subsequently, samples were post-fixed overnight on ice with secondary fixative comprising $2 \%$ glutaraldehyde in $0.1 \mathrm{M}$ sodium cacodylate buffer, $\mathrm{pH}$ 7.2. The next day, samples were washed thrice in sodium cacodylate buffer, before $1 \%$ osmium tetroxide ( $(\mathrm{v} / \mathrm{v})$ in $0.1 \mathrm{M}$ sodium cacodylate buffer) was applied for post-fixation for $1 \mathrm{~h}$ on ice. After $2 \times 10 \mathrm{~min}$ washing steps in sodium cacodylate buffer and three brief washing steps in distilled water, the samples were stained en bloc with $1 \%$ uranyl acetate $((\mathrm{v} / \mathrm{v})$ in distilled water) for $1 \mathrm{~h}$ on ice and briefly washed with distilled water. The dehydration was performed on ice in solutions with increasing ethanol concentrations, and the samples were subsequently infiltrated in Epon resin (100\% EtOH/Epon 1:1 (v/v), 30 and $90 \mathrm{~min} ; 100 \%$ Epon, overnight), placed into embedding molds, and polymerized for $48 \mathrm{~h}$ at $70^{\circ} \mathrm{C}$. $65-\mathrm{nm}$ sections of the embedded samples were obtained approaching from the anterior edge using an ultramicrotome (UC6, Leica Microsystems) and placed on formvar-coated $2 \times 1 \mathrm{~mm}$ copper slot grids. Sections were post-fixed and post-stained with uranyl acetate or uranyl acetate replacement (Science Services, Munich) and lead citrate following standard protocols. Micrographs were acquired with a JEM 1011 electron microscope (JEOL) equipped with a Gatan Orius 1200A camera using the Digital Micrograph software package at a 5,000fold to 12,000-fold magnification.

Electron tomography was essentially performed as described previously (Jung et al, 2015; Strenzke et al, 2016). Briefly, 250-nm sections were applied to formvar-coated copper mesh grids and post-stained as described above. $10 \mathrm{~nm}$ gold beads were applied to both sides of the grid as fiducial markers. Tilt series were acquired with a JEOL electron microscope (JEM 2100) from $-60^{\circ}$ to $+60^{\circ}$ with an increment of $1^{\circ}$ using Serial-EM software. Tomograms were generated using the IMOD package etomo, and models were generated using $3 \mathrm{dmod}$ (bio3d.colorado.edu/imod/).

\section{Auditory brainstem responses}

Recordings of ABRs (auditory brainstem responses) and DPOAE (distortion product otoacoustic emissions) were performed as previously described (Jing et al, 2013). In brief, mice were anesthetized with a combination of ketamine $(125 \mathrm{mg} / \mathrm{kg})$ and xylazine $(2.5 \mathrm{mg} /$ kg) i.p. The core temperature was maintained constant at $37^{\circ} \mathrm{C}$ using a heat blanket (Hugo Sachs Elektronik-Harvard Apparatus). For stimulus generation, presentation, and data acquisition, we used the TDT II system run by BioSig software (Tucker Davis Technologies) (MathWorks). Tone bursts $(4 / 6 / 8 / 12 / 16 / 24 / 32 \mathrm{kHz}, 10 \mathrm{~ms}$ plateau, $1 \mathrm{~ms} \cos ^{2}$ rise/fall) or clicks of $0.03 \mathrm{~ms}$ were presented at $40 \mathrm{~Hz}$ (tone bursts) or $20 \mathrm{~Hz}$ (clicks) in the free field ipsilaterally using a JBL 2402 speaker. The difference potential between vertex and mastoid subdermal needles was amplified 50,000 times, filtered $(400-4,000 \mathrm{~Hz})$, and sampled at a rate of $50 \mathrm{kHz}$ for $20 \mathrm{~ms}, 1,300$ times, to obtain two mean ABR traces for each sound intensity.

\section{Purification of recombinant endophilin-A1}

Plasmid pGEX6P-endopilin-A1 (rat) was a kind gift of Prof. P. De Camilli (Yale University, New Haven CT, USA). After an overnight 
expression $\left(16^{\circ} \mathrm{C}\right.$ for $\sim 9 \mathrm{~h}$ ), in E. coli BL21(DE3) (Sigma) the protein was purified on a GST-glutathione affinity column (GE Healthcare, Piscataway, NJ). The GST-tag was cleaved using PreScission protease (GE Healthcare, Piscataway, NJ), and the sample was then subjected to gel filtration chromatography (Superdex 200 10/300 GL; Amersham Pharmacia Biosciences, Piscataway, NJ) in $20 \mathrm{mM}$ Tris (pH 7.4), $300 \mathrm{mM} \mathrm{KCl}, 5 \mathrm{mM}$ imidazole, $1 \mathrm{mM}$ DTT. The protein concentration was determined $(14.5 \mathrm{mg} / \mathrm{ml})$, and aliquots of the purified protein were stored at $-80^{\circ} \mathrm{C}$ until being used as detailed below.

\section{Immunoprecipitation experiments}

The immunoprecipitation experiments were performed as described in Murdoch et al (2016). Briefly, HeLa cells (ATCC $^{\circledR}$ CCL-2; $1.5 \times 10^{6}$ ) were plated in a $\varnothing 10-\mathrm{cm}$ plate and transfected with pmRFP-endophilin-A1 (a gift of P. De Camilli, Yale University, New Haven, CT, USA) and pEGFP-otoferlin (gift of T. Weber, University Medical Center Göttingen), or pEGFP-N1 (Clontech) for control, using Fugene (Invitrogen) $3 \mathrm{~h}$ after plating (the total DNA amount was $8 \mu \mathrm{g} /$ plate). On average, $75-80 \%$ of HeLa cells were transfected. Cells were harvested $72 \mathrm{~h}$ after transfection and lysed in $10 \mathrm{mM}$ Tris/Cl (pH 7.5), $150 \mathrm{mM} \mathrm{NaCl}, 0.5 \mathrm{mM}$ EDTA, 0.5\% NP40. The lysates were then incubated for $2 \mathrm{~h}$ at $4^{\circ} \mathrm{C}$ with ChromoTek Trap ${ }^{\circledR}$-GFP agarose beads (ChromoTek, Germany) following manufacturer's protocol (GFP-Trap ${ }^{\circledR}$ A A for immunoprecipitation of GFPfusion proteins from mammalian cell extract). After binding, protein GST-Trap_A beads were resuspended in ice-cold dilution buffer and centrifuged. The washing step was repeated twice according to the standard protocol. Fractions (input $=$ cell lysate; bound $=$ beads with bound proteins, non-bound = supernatant) were loaded onto SDS-PAGE gel followed by an immunoblot analysis against endophilin-A1 (Endo A1 antibody, L-18, Santa Cruz, USA), RFP (5F8; ChromoTek, Germany), using standard procedure and Odyssey ${ }^{\circledR}$ imaging system (LI-COR, Lincoln, NE, USA). Three independent experiments were performed.

To check whether otoferlin binds directly to endophilin, HeLa cells $\left(\right.$ ATCC $^{\circledR}$ CCL-2; $1.2 \times 10^{6}$ ) were plated in a $\varnothing 10-\mathrm{cm}$ plate and transfected with pEGFP or pEGFP-otoferlin $(7 \mu \mathrm{g} /$ plate in total) using Fugene (Invitrogen). 75-80\% of HeLa cells were transfected on average. Cells were harvested $72 \mathrm{~h}$ after transfection and lysed in $10 \mathrm{mM}$ Tris/Cl (pH 7.5), $150 \mathrm{mM} \mathrm{NaCl}, 0.5$ mM EDTA, 0.5\% NP-40. EGFP or otoferlin-EGFP was then bound to Trap ${ }^{\circledR}$-GFP agarose beads (ChromoTek) following manufacturer's instructions, and the beads were incubated with $50 \mu \mathrm{g}$ of purified endophilin-A1 protein for $2 \mathrm{~h}$ at $4^{\circ} \mathrm{C}$. After centrifugation $\left(2,500 \mathrm{~g}, 2 \mathrm{~min}, 4^{\circ} \mathrm{C}\right)$, two fractions were obtained: beads with bound proteins (bound) and supernatant (nonbound). In addition to cell lysates (input), these fractions were loaded onto SDS-PAGE gel followed by an immunoblot analysis against endophilin-A1 (EndoA1 antibody L-18, Santa Cruz, USA) using standard procedure and Odyssey ${ }^{\circledR}$ imaging system (LI-COR, Lincoln, NE, USA). Two independent experiments were made.

\section{Data analysis}

\section{Immunohistochemistry}

IHC synaptic ribbons were manually counted in confocal $z$-projections of immunolabeled organs of Corti using ImageJ software.
Analysis of $\mathrm{Ca}_{V} 1.3$ STED imaging data was performed in Igor Pro 6.3 software (Wavemetrics). The area of spot- and line-shaped $\mathrm{Ca}^{2+}$ channel clusters was estimated by fitting a 2D Gaussian function (genetic fit algorithm; Sanchez del Rio \& Pareschi, 2001) to the individual clusters in 2D STED images to obtain the full width at half maximum (FWHM) of the long and short axes. Subsequently, areas of the clusters were calculated by the following formula: area $=\pi \times($ long axis $/ 2) \times($ short axis $/ 2)$. Otoferlin levels of IHCs were semi-quantitatively assessed as immunofluorescence intensity values and analyzed using Imaris (Bitplane) and MATLAB (MathWorks) as described before (Strenzke et al, 2016). Otoferlin intensity line scans along the longitudinal axis of IHCs were generated from single sections using ImageJ; Igor Pro 6.3 software was used to generate average intensity profiles after normalizing the intensity profiles of the individual cells to their lengths.

\section{Patch-clamp electrophysiology}

Electrophysiological data were analyzed using custom-written programs in Igor Pro 6.3. For analysis of IV curves, the last $5 \mathrm{~ms}$ of the evoked $\mathrm{Ca}^{2+}$ current was averaged. For measurements of exocytosis, total $\mathrm{Ca}^{2+}$ charge $\left(\mathrm{Q}_{\mathrm{Ca}}\right)$ was estimated by taking the integral of the leak-subtracted current during the depolarization step and the exocytic increment in capacitance $\left(\Delta \mathrm{C}_{\mathrm{m}}\right)$ was quantified as the difference in the averaged $C_{m} 400 \mathrm{~ms}$ before and after (skipping the first 60 or $100 \mathrm{~ms}$ ) the depolarization. Mean estimates of individual IHCs were calculated from two to four rounds of exocytosis, and were used to calculate grand averages of IHCs per genotype. This avoided dominance of IHCs contributing more sweeps. Only IHCs with reproducible $\Delta \mathrm{C}_{\mathrm{m}}$ among the individual rounds were included.

Endocytosis was assessed as described previously (Neef et al, 2014). IHCs showed a slow depolarization-independent decrease in $\mathrm{C}_{\mathrm{m}}$ during the perforated patch-clamp recording. To correct for this, we used two independent methods: (i) by fitting and subtracting an exponential function to the baseline $\mathrm{C}_{\mathrm{m}}$ as recorded during the entire experiment (Fig EV4A, $\mathrm{C}$ and $\mathrm{C}^{\prime}$ ) or (ii) by fitting and subtracting a linear function to the last $10 \mathrm{~s}$ of a non-depolarizing pulse recorded before the actual trace (Fig EV4D and $\mathrm{D}^{\prime}$ ). The results obtained using both methods were comparable (Fig EV4C" and $\mathrm{D}^{\prime \prime}$ ). We favor the first method, which seems to perform a better correction, evident from the least divergence of the recorded traces for each individual IHC (for an example, see Fig EV4C).

The endocytic decline in $\mathrm{C}_{\mathrm{m}}$ following a depolarization-induced exocytic $\Delta \mathrm{C}_{\mathrm{m}}$ was measured over $20 \mathrm{~s}$ following a 2-s-long recording of $C_{m}$ baseline and 20 or $200 \mathrm{~ms}$ of depolarization. After correction, we determined the residual slope of the $2 \mathrm{~s}$ preceding the depolarization (residual pre-slope) and excluded those IHCs where the residual pre-slope exceeded the average \pm 2 times the standard deviation for each genotype (for $20 \mathrm{~ms}$, one cell was excluded for each genotype; for $200 \mathrm{~ms}$, one cell was excluded for the Wt and for the $1 / 3$-DKO). Even though the residual pre-slope exhibits a negative trend (for $20 \mathrm{~ms}$ : $-0.51 \pm 0.24 \mathrm{fF} / \mathrm{s}$ for $\mathrm{Wt},-0.20 \pm 0.28 \mathrm{fF} / \mathrm{s}$ for $1 / 2$-DKO, and $-0.62 \pm 0.22 \mathrm{fF} / \mathrm{s}$ for $1 / 3$-DKO; for $200 \mathrm{~ms}$ : $-0.27 \pm 0.37 \mathrm{fF} / \mathrm{s}$ for $\mathrm{Wt},-0.28 \pm 0.23 \mathrm{fF} / \mathrm{s}$ for $1 / 2-\mathrm{DKO}$, and $0.036 \pm 0.22 \mathrm{fF} / \mathrm{s}$ for $1 / 3-\mathrm{DKO}$ ), it is not statistically different between the genotypes (for $20 \mathrm{~ms}$ : one-way ANOVA, $F(2,35)=$ $0.80, P=0.4507$; for $200 \mathrm{~ms}$ : one-way ANOVA, $F(2,36)=0.45$, $P=0.6391)$. 
Analysis of the linear component of endocytosis following brief depolarizations (20 ms) was performed by fitting a linear function to the post-depolarization $\mathrm{C}_{\mathrm{m}}$ data, skipping the first $200 \mathrm{~ms}$, and noting the point at which the line fit or its extrapolation in time returned to baseline $C_{m}$. Responses in which $C_{m}$ did not return to baseline within $80 \mathrm{~s}$ were excluded from this analysis. The number of IHCs where $\mathrm{C}_{\mathrm{m}}$ did not return to baseline was low for all genotypes: Wt (3 out of 17 recorded IHCs), 1/2-DKO ( 0 cells out of 14), and $1 / 3$-DKO ( 3 cells out of 17 ), but many IHCs regardless of genotype showed at least one trace without $\mathrm{C}_{\mathrm{m}}$ return within $80 \mathrm{~s}$.

We analyzed the exponential component of $\mathrm{C}_{\mathrm{m}}$ decline following 200-ms-long depolarization by fitting a linear function to the final $10 \mathrm{~s}$ of $\mathrm{C}_{\mathrm{m}}$ data and subtracting the extrapolated fit. The residual was then fitted with an exponential function using a genetic curve fitting algorithm (Sanchez del Rio \& Pareschi, 2001). In few cases, the fitting window for the linear component was slightly adjusted to exclude $\mathrm{C}_{\mathrm{m}}$ artifacts. For some cells, the $\mathrm{C}_{\mathrm{m}}$ decline did not exhibit an exponential component ( 1 out of 12 IHCs for Wt, 3 out of 13 for $1 / 2$-DKO, and 3 out of 14 for $1 / 3-\mathrm{DKO})$. Therefore, they were excluded from the statistical analysis of the amplitude or time constant of the exponential component; yet, they are still included for the slope of the linear component for $200 \mathrm{~ms}$.

\section{Auditory brainstem recordings}

Recordings were performed as described (Jing et al, 2013). In brief, hearing threshold was determined with $10 \mathrm{~dB}$ precision as the lowest stimulus intensity that evoked a reproducible response waveform in both traces by visual inspection.

\section{Transmission electron microscopy (TEM)}

Quantification of electron microscopy data was performed with ImageJ for random sections, and with IMOD software for tomograms. In random sections, the ribbon proximity (Fig 7) was defined as area around the ribbon in $1 \mu \mathrm{m}$ distance from the center of the synaptic ribbon. For each section, the exact area of the intracellular cytoplasm was measured, whereby the area of mitochondria was excluded. Numbers of small, clear vesicles (max. diameter $70 \mathrm{~nm}$ between outer leaflets of the vesicle membrane; Figs 7B and 9D) except of the vesicles in the first row around the ribbon-were counted and divided by the respective area. For quantification of endosome-like vacuoles (ELVs), the area of all ELVs per section was cumulated and divided by the cytoplasmic area (Figs 7C and 9E). For coated structures, the following subgroups were defined: (i) coated vesicles with a maximum outer diameter of $70 \mathrm{~nm}$; (ii) coated vacuoles with an outer diameter of more than $70 \mathrm{~nm}$ as well as tubular structures fully covered by a coat; (iii) coated pits containing a connection to the plasma membrane; and (iv) coated pits on otherwise uncoated ELVs. For quantification of SVs around the ribbon, the following analysis criteria were used for random sections: Ribbon height was measured from the top of the ribbon to the bottom excluding the presynaptic density; the distal half of the ribbon was defined as the upper $50 \%$ of the total ribbon length. SVs were allocated to the distal or proximal halves of the ribbon if more than $50 \%$ of the SV area were located in the respective region (Fig $8 \mathrm{C}^{\prime}$ ). Moreover, two distinct SV pools were defined: (i) The ribbon-associated pool contained all SVs in the first row around the ribbon with a maximum distance of $80 \mathrm{~nm}$ (RA-SVs, Figs $8 \mathrm{C}$ and 9B) and (ii) the membrane-proximal SV pool (MP-SVs), which was formed by SVs in the first layer above the AZ membrane within a maximum distance of $<25 \mathrm{~nm}$ to the plasma membrane and a maximum lateral distance of $<100 \mathrm{~nm}$ to the presynaptic density (Figs 8D and 9C).

\section{Electron tomography}

For quantification of tomograms, the definition of MP-SVs was adjusted to include all SVs with a maximal distance of $50 \mathrm{~nm}$ to the AZ membrane that were no more than $100 \mathrm{~nm}$ apart from the presynaptic density (all criteria being valid at the maximum projection of the respective SV in the tomogram, no changes to RA-SV definition). This adjustment was made not only to guarantee for comparability with other recent ultrastructural studies of SV pools at ribbon synapses, but also because the high z-resolution of virtual sections from tomograms facilitates the identification of tethers (also including lengths $>25 \mathrm{~nm}$ ) linking MP-SVs to the presynaptic density and to the AZ membrane. Since $65-\mathrm{nm}$ random sections do not provide visualization of tethers, we chose a stricter criterion of $25 \mathrm{~nm}$ maximal distance to avoid over-interpretation of the MP-SV pool. Nevertheless, we re-quantified all tomography data also with the criteria used for random sections resulting in overall unchanged significance values. For quantification of ELVs, the minimal distance between ELV and ribbon was measured; only ELVs within a distance of $200 \mathrm{~nm}$ were included. All distances for random sections and for tomograms were measured between the outer membrane of SVs and the respective structure.

\section{Experimental design and statistical analysis}

Sample sizes were chosen according to typical observation numbers used on each respective field (e.g., immunohistochemistry, cellular or systems electrophysiology, electron microscopy), and can be found both in the respective figures and in the corresponding figure legends. Data were analyzed using MATLAB (MathWorks), Excel (Microsoft), Igor Pro 6 (Wavemetrics), Origin 9.0 (Microcal software), and GraphPad Prism (GraphPad software). Averages are expressed as mean $\pm \mathrm{SEM}$ or mean $\pm \mathrm{SD}$, as specified. Data sets were tested for normal distribution (Jarque-Bera test, D'Agostino and Pearson omnibus normality test, or the Shapiro-Wilk test) and equality of variances. Statistical significance was calculated using one-way ANOVA test followed by Tukey's test for normally distributed data, or Kruskal-Wallis $(\mathrm{K}-\mathrm{W})$ test followed by post hoc Dunn's test for non-normally distributed data. Significant differences are reported as $* P<0.05,{ }^{*} P P<0.01$, and $* * * P<0.001$. Box plots are drawn from 25 to $75 \%$, whiskers indicate $10-90 \%$ of data points, and squares show the averages. For the endocytosis data (Fig 6), the ROUT method from GraphPad Prism was used to identify outliers $(\mathrm{Q}=1 \%)$.

Expanded View for this article is available online.

\section{Acknowledgements}

We thank N. Dietrich, T. Goldak, S. Gerke, C. Senger-Freitag, and M. Costa for expert technical assistance. This work was supported by grants of the German Research Foundation through the collaborative research center 889 (project A8 to I.M. and T.M. and A7 to C.W.), the Emmy-Noether program (to I.M.), and the Leibniz program (to T.M.). C.V. is a Creutzfeldt Fellow of the Elisabeth and Helmut Uhl Foundation. L.M.J.T received an Erasmus Mundus scholarshipNeurasmus. I.K. receives a fellowship from the Engelhorn Stiftung. 


\section{Author contributions}

IM, CW, and TM designed the study. JK performed electron microscopy, electron tomography, and immunohistochemistry followed by confocal and STED microscopy, and handled mouse breeding. LMJT performed patch-clamp capacitance measurements and immunohistochemistry, and contributed to single-cell real-time PCR. CV performed organ of Corti cultures, patch-clamp capacitance measurements, immunohistochemistry and confocal microscopy, as well as Western blots, and contributed to single-cell real-time PCR. JN performed patch-clamp capacitance measurements and contributed to singlecell real-time PCR. IM and MK established and maintained endophilin colonies and performed single-cell real-time PCR. IM and IK performed biochemical interaction studies. CW supervised electron microscopy and tomography. NS supervised auditory systems physiology. All authors performed data analysis. TM, IM, JK, LMJT, and CV prepared the manuscript with contributions of all other authors.

\section{Conflict of interest}

The authors declare that they have no conflict of interest.

\section{References}

Anggono V, Robinson PJ (2007) Syndapin I and endophilin I bind overlapping proline-rich regions of dynamin I: role in synaptic vesicle endocytosis. J Neurochem 102: $931-943$

Bai J, Hu Z, Dittman JS, Pym ECG, Kaplan JM (2010) Endophilin functions as a membrane-bending molecule and is delivered to endocytic zones by exocytosis. Cell 143: $430-441$

Beutner D, Voets T, Neher E, Moser T (2001) Calcium dependence of exocytosis and endocytosis at the cochlear inner hair cell afferent synapse. Neuron 29: 681-690

Boumil RM, Letts VA, Roberts MC, Lenz C, Mahaffey CL, Zhang Z, Moser T, Frankel WN (2010) A missense mutation in a highly conserved alternate exon of dynamin-1 causes epilepsy in fitful mice. PLoS Genet 6: e1001046

Brandt A, Striessnig J, Moser T (2003) CaV1.3 channels are essential for development and presynaptic activity of cochlear inner hair cells. J Neurosci 23: 10832-10840

Brandt A, Khimich D, Moser T (2005) Few CaV1.3 channels regulate the exocytosis of a synaptic vesicle at the hair cell ribbon synapse. J Neurosci 25: $11577-11585$

Chen Y, Deng L, Maeno-Hikichi Y, Lai M, Chang S, Chen G, Zhang J (2003) Formation of an endophilin- $\mathrm{Ca}^{2+}$ channel complex is critical for clathrinmediated synaptic vesicle endocytosis. Cell 115: 37-48

Duncker SV, Franz C, Kuhn S, Schulte U, Campanelli D, Brandt N, Hirt B, Fakler B, Blin N, Ruth P, Engel J, Marcotti W, Zimmermann U, Knipper M (2013) Otoferlin couples to clathrin-mediated endocytosis in mature cochlear inner hair cells. J Neurosci 33: 9508-9519

Ferguson S, Raimondi A, Paradise S, Shen H, Mesaki K, Ferguson A, Destaing O, Ko G, Takasaki J, Cremona O, O’Toole E, De Camilli P (2009) Coordinated actions of actin and BAR proteins upstream of dynamin at endocytic clathrin-coated pits. Deu Cell 17: 811-822

Ferguson SM, De Camilli P (2012) Dynamin, a membrane-remodelling GTPase. Nat Reu Mol Cell Biol 13: 75-88

Frank T, Khimich D, Neef A, Moser T (2009) Mechanisms contributing to synaptic $\mathrm{Ca}^{2+}$ signals and their heterogeneity in hair cells. Proc Natl Acad Sci USA 106: $4483-4488$

Frank T, Rutherford MA, Strenzke N, Neef A, Pangršič T, Khimich D, Fejtova A, Fetjova A, Gundelfinger ED, Liberman MC, Harke B, Bryan KE, Lee A, Egner
A, Riedel D, Moser T (2010) Bassoon and the synaptic ribbon organize $\mathrm{Ca}^{2+}$ channels and vesicles to add release sites and promote refilling. Neuron 68: $724-738$

Gad H, Ringstad N, Löw P, Kjaerulff O, Gustafsson J, Wenk M, Di Paolo G, Nemoto Y, Crum J, Ellisman MH, De Camilli P, Shupliakov O, Brodin L (2000) Fission and uncoating of synaptic clathrin-coated vesicles are perturbed by disruption of interactions with the SH3 domain of endophilin. Neuron 27: 301-312

Guillet M, Sendin G, Bourien J, Puel J-L, Nouvian R (2016) Actin filaments regulate exocytosis at the hair cell ribbon synapse. J Neurosci 36: 649-654

Haucke V, Neher E, Sigrist SJ (2011) Protein scaffolds in the coupling of synaptic exocytosis and endocytosis. Nat Reu Neurosci 12: 127-138

jing Z, Rutherford MA, Takago H, Frank T, Fejtova A, Khimich D, Moser T, Strenzke N (2013) Disruption of the presynaptic cytomatrix protein bassoon degrades ribbon anchorage, multiquantal release, and sound encoding at the hair cell afferent synapse. J Neurosci 33: 4456-4467

Jung S, Maritzen T, Wichmann C, Jing Z, Neef A, Revelo NH, Al-Moyed H, Meese S, Wojcik SM, Panou I, Bulut H, Schu P, Ficner R, Reisinger E, Rizzoli So, Neef J, Strenzke N, Haucke V, Moser T (2015) Disruption of adaptor protein $2 \mu$ (AP- $2 \mu)$ in cochlear hair cells impairs vesicle reloading of synaptic release sites and hearing. EMBO J 34: 2686-2702

Kamin D, Revelo NH, Rizzoli SO (2014) FM dye photo-oxidation as a tool for monitoring membrane recycling in inner hair cells. PLOS ONE 9: e88353

Khimich D, Nouvian R, Pujol R, tom Dieck S, Egner A, Gundelfinger ED, Moser $T$ (2005) Hair cell synaptic ribbons are essential for synchronous auditory signalling. Nature 434: 889-894

Kokotos AC, Cousin MA (2015) Synaptic vesicle generation from central nerve terminal endosomes. Traffic 16: 229-240

Kononenko NL, Puchkov D, Classen GA, Walter AM, Pechstein A, Sawade L, Kaempf N, Trimbuch T, Lorenz D, Rosenmund C, Maritzen T, Haucke V (2014) Clathrin/AP-2 mediate synaptic vesicle reformation from endosome-like vacuoles but are not essential for membrane retrieval at central synapses. Neuron 82: 981-988

Kononenko NL, Haucke V (2015) Molecular mechanisms of presynaptic membrane retrieval and synaptic vesicle reformation. Neuron 85: 484-496

Lenzi D, Crum J, Ellisman MH, Roberts WM (2002) Depolarization redistributes synaptic membrane and creates a gradient of vesicles on the synaptic body at a ribbon synapse. Neuron 36: 649-659

Lindau M, Neher E (1988) Patch-clamp techniques for time-resolved capacitance measurements in single cells. Pflügers Archiv 411: 137-146

Melcher JR, Guinan JH Jr, Knudson IM, Kiang NYS (1996) Generators of the brainstem auditory evoked potential in cat II. Correlating lesion sites with waveform changes. Hear Res 93: 28-51

Meyer AC, Frank T, Khimich D, Hoch G, Riedel D, Chapochnikov NM, Yarin YM, Harke B, Hell SW, Egner A, Moser T (2009) Tuning of synapse number, structure and function in the cochlea. Nat Neurosci 12: 444-453

Mikaelian D, Ruben RJ (1965) Development of hearing in the normal Cba-J mouse: correlation of physiological observations with behavioral responses and with cochlear anatomy. Acta Otolaryngol 59: $451-461$

Milosevic I, Giovedi S, Lou X, Raimondi A, Collesi C, Shen H, Paradise S, O'Toole E, Ferguson S, Cremona O, De Camilli P (2011) Recruitment of endophilin to clathrin-coated pit necks is required for efficient vesicle uncoating after fission. Neuron 72: 587-601

Milosevic I (2018) Revisiting the role of clathrin-mediated endoytosis in synaptic vesicle recycling. Front Cell Neurosci 12: 27

Moser T, Beutner D (2000) Kinetics of exocytosis and endocytosis at the cochlear inner hair cell afferent synapse of the mouse. Proc Natl Acad Sci USA 97: 883-888 
Murdoch JD, Rostosky CM, Gowrisankaran S, Arora AS, Soukup S-F, Vidal R, Capece V, Freytag S, Fischer A, Verstreken P, Bonn S, Raimundo N, Milosevic I (2016) Endophilin-A deficiency induces the Foxo3a-Fbxo32 network in the brain and causes dysregulation of autophagy and the ubiquitin-proteasome system. Cell Rep 17: 1071-1086

Neef J, Jung S, Wong AB, Reuter K, Pangrsic T, Chakrabarti R, Kugler S, Lenz C, Nouvian R, Boumil RM, Frankel WN, Wichmann C, Moser T (2014) Modes and regulation of endocytic membrane retrieval in mouse auditory hair cells. J Neurosci 34: 705-716

Neef J, Urban NT, Ohn T-L, Frank T, Jean P, Hell SW, Willig KI, Moser T (2018) Quantitative optical nanophysiology of $\mathrm{Ca}^{2+}$ signaling at inner hair cell active zones. Nat Commun 9: 290

Nouvian R, Neef J, Bulankina AV, Reisinger E, Pangršič T, Frank T, Sikorra S, Brose N, Binz T, Moser T (2011) Exocytosis at the hair cell ribbon synapse apparently operates without neuronal SNARE proteins. Nat Neurosci 14: $411-413$

Ohn T-L, Rutherford MA, Jing Z, Jung S, Duque-Afonso CJ, Hoch G, Picher MM, Scharinger A, Strenzke N, Moser T (2016) Hair cells use active zones with different voltage dependence of $\mathrm{Ca}^{2+}$ influx to decompose sounds into complementary neural codes. Proc Natl Acad Sci USA 113: E4716-E4725

Pangrsic T, Lasarow L, Reuter K, Takago H, Schwander M, Riedel D, Frank T, Tarantino LM, Bailey JS, Strenzke N, Brose N, Müller U, Reisinger E, Moser $T$ (2010) Hearing requires otoferlin-dependent efficient replenishment of synaptic vesicles in hair cells. Nat Neurosci 13: 869-876

Pangrsic T, Vogl C (2018) Balancing presynaptic release and endocytic membrane retrieval at hair cell ribbon synapses. FEBS Lett 592: 3633-3650

Parsons TD, Lenzi D, Almers W, Roberts WM (1994) Calcium-triggered exocytosis and endocytosis in an isolated presynaptic cell: capacitance measurements in saccular hair cells. Neuron 13: 875-883

Pechstein A, Gerth F, Milosevic I, Jäpel M, Eichhorn-Grünig M, Vorontsova O, Bacetic J, Maritzen T, Shupliakov O, Freund C, Haucke V (2015) Vesicle uncoating regulated by SH3-SH3 domain-mediated complex formation between endophilin and intersectin at synapses. EMBO Rep 16: 232-239

Perera RM, Zoncu R, Lucast L, Camilli PD, Toomre D (2006) Two synaptojanin 1 isoforms are recruited to clathrin-coated pits at different stages. Proc Natl Acad Sci USA 103: 19332-19337

Platzer J, Engel J, Schrott-Fischer A, Stephan K, Bova S, Chen H, Zheng H, Striessnig J (2000) Congenital deafness and sinoatrial node dysfunction in mice lacking class D L-type $\mathrm{Ca}^{2+}$ channels. Cell 102: 89-97

Ramakrishnan NA, Drescher MJ, Drescher DG (2009) Direct interaction of otoferlin with syntaxin 1A, SNAP-25, and the L-type voltage-gated calcium channel Cav1.3. J Biol Chem 284: 1364-1372

Reisinger E, Bresee C, Neef J, Nair R, Reuter K, Bulankina A, Nouvian R, Koch M, Bückers J, Kastrup L, Roux I, Petit C, Hell SW, Brose N, Rhee J-S, Kügler S, Brigande JV, Moser T (2011) Probing the functional equivalence of otoferlin and synaptotagmin 1 in exocytosis. J Neurosci 31: 4886-4895

Renard H-F, Simunovic M, Lemière J, Boucrot E, Garcia-Castillo MD, Arumugam S, Chambon V, Lamaze C, Wunder C, Kenworthy AK, Schmidt AA, McMahon HT, Sykes C, Bassereau P, Johannes L (2015) Endophilin-A2 functions in membrane scission in clathrin-independent endocytosis. Nature 517: $493-496$

Revelo NH, Kamin D, Truckenbrodt S, Wong AB, Reuter-Jessen K, Reisinger E, Moser T, Rizzoli SO (2014) A new probe for super-resolution imaging of membranes elucidates trafficking pathways. J Cell Biol 205: 591-606

Ringstad N, Gad H, Löw P, Paolo GD, Brodin L, Shupliakov O, Camilli PD (1999) Endophilin/SH3p4 is required for the transition from early to late stages in clathrin-mediated synaptic vesicle endocytosis. Neuron 24: $143-154$
Roux I, Safieddine S, Nouvian R, Grati M, Simmler M-C, Bahloul A, Perfettini I, Le Gall M, Rostaing P, Hamard G, Triller A, Avan P, Moser T, Petit C (2006) Otoferlin, defective in a human deafness form, is essential for exocytosis at the auditory ribbon synapse. Cell 127: 277-289

Roux I, Hosie S, Johnson SL, Bahloul A, Cayet N, Nouaille S, Kros C], Petit C, Safieddine $S$ (2009) Myosin VI is required for the proper maturation and function of inner hair cell ribbon synapses. Hum Mol Genet 18: 4615- 4628

Rutherford MA, Moser T (2016) The ribbon synapse between type I spiral ganglion neurons and inner hair cells. In The primary auditory neurons of the mammalian cochlea, Dabdoub A, Fritzsch B, Popper AN, Fay RR (eds), pp 117-156. Springer Handbook of Auditory Research. New York, NY: Springer

Safieddine S, El-Amraoui A, Petit C (2012) The auditory hair cell ribbon synapse: from assembly to function. Annu Reu Neurosci 35: 509-528

Saheki Y, Camilli PD (2012) Synaptic vesicle endocytosis. Cold Spring Harb Perspect Biol 4: a005645

Sanchez del Rio M, Pareschi G (2001) Global optimization and reflectivity data fitting for x-ray multilayer mirrors by means of genetic algorithms. In Proc. SPIE 4145, Aduances in X-Ray Optics. https://doi.org/10.1117/12.411624

Schuske KR, Richmond JE, Matthies DS, Davis WS, Runz S, Rube DA, van der Bliek AM, Jorgensen EM (2003) Endophilin is required for synaptic vesicle endocytosis by localizing synaptojanin. Neuron 40: 749-762

Shnerson A, Pujol R (1981) Age-related changes in the C57BL/6J mouse cochlea. I. Physiological findings. Deu Brain Res 2: 65-75

Siegel JH, Brownell WE (1986) Synaptic and Golgi membrane recycling in cochlear hair cells. J Neurocytol 15: 311-328

Simunovic M, Manneville J-B, Renard H-F, Evergren E, Raghunathan K, Bhatia D, Kenworthy AK, Voth GA, Prost J, McMahon HT, Johannes L, Bassereau P, Callan-jones A (2017) Friction mediates scission of tubular membranes scaffolded by BAR proteins. Cell 170: 172-184.e11

Sobkowicz HM, Rose JE, Scott GE, Slapnick SM (1982) Ribbon synapses in the developing intact and cultured organ of Corti in the mouse. J Neurosci 2: 942-957

Soda K, Balkin DM, Ferguson SM, Paradise S, Milosevic I, Giovedi S, VolpicelliDaley L, Tian X, Wu Y, Ma H, Son SH, Zheng R, Moeckel G, Cremona O, Holzman LB, De Camilli P, Ishibe S (2012) Role of dynamin, synaptojanin, and endophilin in podocyte foot processes. J Clin Invest 122: 4401-4411

Strenzke N, Chanda S, Kopp-Scheinpflug C, Khimich D, Reim K, Bulankina AV, Neef A, Wolf F, Brose N, Xu-Friedman MA, Moser T (2009) Complexin-I is required for high-fidelity transmission at the endbulb of Held auditory synapse. J Neurosci 29: 7991-8004

Strenzke N, Chakrabarti R, Al-Moyed H, Müller A, Hoch G, Pangrsic T, Yamanbaeva G, Lenz C, Pan K-T, Auge E, Geiss-Friedlander R, Urlaub H, Brose N, Wichmann C, Reisinger E (2016) Hair cell synaptic dysfunction, auditory fatigue and thermal sensitivity in otoferlin Ile515Thr mutants. EMBO J 35: 2519-2535

Sundborger A, Soderblom C, Vorontsova O, Evergren E, Hinshaw JE, Shupliakov O (2011) An endophilin-dynamin complex promotes budding of clathrin-coated vesicles during synaptic vesicle recycling. J Cell Sci 124: $133-143$

Trapani JG, Obholzer N, Mo W, Brockerhoff SE, Nicolson T (2009) Synaptojanin1 is required for temporal fidelity of synaptic transmission in hair cells. PLoS Genet 5: e1000480

Vehlow A, Soong D, Vizcay-Barrena G, Bodo C, Law A-L, Perera U, Krause M (2013) Endophilin, lamellipodin, and mena cooperate to regulate F-actindependent EGF-receptor endocytosis. EMBO J 32: 2722 - 2734

Verstreken P, Kjaerulff O, Lloyd TE, Atkinson R, Zhou Y, Meinertzhagen IA, Bellen HJ (2002) Endophilin mutations block clathrin-mediated endocytosis but not neurotransmitter release. Cell 109: 101-112 
Verstreken P, Koh T-W, Schulze KL, Zhai RG, Hiesinger PR, Zhou Y, Mehta SQ, Cao Y, Roos J, Bellen HJ (2003) Synaptojanin is recruited by endophilin to promote synaptic vesicle uncoating. Neuron 40: 733-748

Vincent PF, Bouleau Y, Petit C, Dulon D (2015) A synaptic F-actin network controls otoferlin-dependent exocytosis in auditory inner hair cells. Elife 4: e10988

Vogl C, Cooper BH, Neef J, Wojcik SM, Reim K, Reisinger E, Brose N, Rhee J-S, Moser T, Wichmann C (2015) Unconventional molecular regulation of synaptic vesicle replenishment in cochlear inner hair cells. J Cell Sci 128: $638-644$

Vogl C, Panou I, Yamanbaeva G, Wichmann C, Mangosing SJ, Vilardi F, Indzhykulian AA, Pangršič T, Santarelli R, Rodriguez-Ballesteros M, Weber T, Jung S, Cardenas E, Wu X, Wojcik SM, Kwan KY, Del Castillo I, Schwappach B, Strenzke N, Corey DP et al (2016) Tryptophan-rich basic protein (WRB) mediates insertion of the tail-anchored protein otoferlin and is required for hair cell exocytosis and hearing. EMBO J 35: $2536-2552$

Vogl C, Butola T, Haag N, Hausrat TJ, Leitner MG, Moutschen M, Lefèbvre PP, Speckmann C, Garrett L, Becker L, Fuchs H, Hrabe de Angelis M, Nietzsche
S, Kessels MM, Oliver D, Kneussel M, Kilimann MW, Strenzke N (2017) The $B E A C H$ protein $L R B A$ is required for hair bundle maintenance in cochlear hair cells and for hearing. EMBO Rep 18: 2015-2029

Watanabe S, Mamer LE, Raychaudhuri S, Luvsanjav D, Eisen J, Trimbuch T, Söhl-Kielczynski B, Fenske P, Milosevic I, Rosenmund C, Jorgensen EM (2018) Synaptojanin and endophilin mediate neck formation during ultrafast endocytosis. Neuron 98: 1184-1197.e6

Weiler S, Krinner S, Wong AB, Moser T, Pangršič T (2014) ATP hydrolysis is critically required for function of CaV1.3 channels in cochlear inner hair cells via fueling $\mathrm{Ca}^{2+}$ clearance. J Neurosci 34: 6843-6848

Wichmann C, Moser T (2015) Relating structure and function of inner hair cell ribbon synapses. Cell Tissue Res 361: 95-114

Wong AB, Rutherford MA, Gabrielaitis M, Pangršič T, Göttfert F, Frank T, Michanski S, Hell S, Wolf F, Wichmann C, Moser T (2014) Developmental refinement of hair cell synapses tightens the coupling of $\mathrm{Ca}^{2+}$ influx to exocytosis. EMBO J 33: 247-264

Yang Y, Wei M, Xiong Y, Du X, Zhu S, Yang L, Zhang C, Liu J-J (2015) Endophilin $A 1$ regulates dendritic spine morphogenesis and stability through interaction with p140Cap. Cell Res 25: 496-516 
